

\section{Algebra \& Number Theory}

www.jant.org

\section{EDITORS}

\author{
MANAGING EDITOR \\ Bjorn Poonen \\ Massachusetts Institute of Technology \\ Cambridge, USA
}

\author{
EDITORIAL BOARD CHAIR \\ David Eisenbud \\ University of California \\ Berkeley, USA
}

\section{BOARD OF EDITORS}

Georgia Benkart

Dave Benson

Richard E. Borcherds

John H. Coates

J-L. Colliot-Thélène

Brian D. Conrad

Hélène Esnault

Hubert Flenner

Edward Frenkel

Andrew Granville

Joseph Gubeladze

Ehud Hrushovski

Craig Huneke

Mikhail Kapranov

Yujiro Kawamata

János Kollár

Hendrik W. Lenstra

Yuri Manin

Barry Mazur
University of Wisconsin, Madison, USA

University of Aberdeen, Scotland

University of California, Berkeley, USA

University of Cambridge, UK

CNRS, Université Paris-Sud, France

University of Michigan, USA

Universität Duisburg-Essen, Germany

Ruhr-Universität, Germany

University of California, Berkeley, USA

Université de Montréal, Canada

San Francisco State University, USA

Hebrew University, Israel

University of Kansas, USA

Yale University, USA

University of Tokyo, Japan

Princeton University, USA

Universiteit Leiden, The Netherlands

Northwestern University, USA

Harvard University, USA
Susan Montgomery

Shigefumi Mori

Andrei Okounkov

Raman Parimala

Victor Reiner

Karl Rubin

Peter Sarnak

Michael Singer

Ronald Solomon

Vasudevan Srinivas

J. Toby Stafford

Bernd Sturmfels

Richard Taylor

Ravi Vakil

Michel van den Bergh

Marie-France Vignéras

Kei-Ichi Watanabe

Andrei Zelevinsky

Efim Zelmanov
University of Southern California, USA

RIMS, Kyoto University, Japan

Princeton University, USA

Emory University, USA

University of Minnesota, USA

University of California, Irvine, USA

Princeton University, USA

North Carolina State University, USA

Ohio State University, USA

Tata Inst. of Fund. Research, India

University of Michigan, USA

University of California, Berkeley, USA

Harvard University, USA

Stanford University, USA

Hasselt University, Belgium

Université Paris VII, France

Nihon University, Japan

Northeastern University, USA

University of California, San Diego, USA

\section{PRODUCTION}

ant@mathscipub.org

Paulo Ney de Souza, Production Manager

Silvio Levy, Senior Production Editor

See inside back cover or www.jant.org for submission instructions.

Regular subscription rate for 2009: \$200.00 a year (\$140.00 electronic only).

Subscriptions, requests for back issues from the last three years and changes of subscribers address should be sent to Mathematical Sciences Publishers, Department of Mathematics, University of California, Berkeley, CA 94720-3840, USA.

Algebra \& Number Theory, ISSN 1937-0652, at Mathematical Sciences Publishers, Department of Mathematics, University of California, Berkeley, CA 94720-3840 is published continuously online. Periodical rate postage paid at Berkeley, CA 94704, and additional mailing offices.

\section{PUBLISHED BY \\ athematical sciences publishers \\ http://www.mathscipub.org}

A NON-PROFIT CORPORATION

Typeset in IATEX

Copyright $\odot 2009$ by Mathematical Sciences Publishers 


\title{
Cox rings of degree one del Pezzo surfaces
}

\author{
Damiano Testa, Anthony Várilly-Alvarado and Mauricio Velasco
}

Let $X$ be a del Pezzo surface of degree one over an algebraically closed field, and let $\operatorname{Cox}(X)$ be its total coordinate ring. We prove the missing case of a conjecture of Batyrev and Popov, which states that $\operatorname{Cox}(X)$ is a quadratic algebra. We use a complex of vector spaces whose homology determines part of the structure of the minimal free $\operatorname{Pic}(X)$-graded resolution of $\operatorname{Cox}(X)$ over a polynomial ring. We show that sufficiently many Betti numbers of this minimal free resolution vanish to establish the conjecture.

\section{Introduction}

Let $k$ be an algebraically closed field and let $X$ be a smooth projective integral scheme over $k$. Assume that the Picard group $\operatorname{Pic}(X)$ is freely generated by the classes of divisors $D_{0}, D_{1}, \ldots, D_{r}$. The total coordinate ring, or Cox ring of $X$ with respect to this basis is given by

$$
\operatorname{Cox}(X):=\bigoplus_{\left(m_{0}, \ldots, m_{r}\right) \in \mathbb{Z}^{r+1}} \mathrm{H}^{0}\left(X, O_{X}\left(m_{0} D_{0}+\cdots+m_{r} D_{r}\right)\right),
$$

with multiplication induced by the multiplication of functions in $k(X)$. Different choices of bases yield (noncanonically) isomorphic Cox rings.

The first appearance of Cox rings was in the context of toric varieties in [Cox 1995]. In that paper Cox proves that if $X$ is a toric variety then its total coordinate ring is a finitely generated multigraded polynomial ring, and that $X$ can be recovered as a quotient of an open subset of $\operatorname{Spec}(\operatorname{Cox}(X))$ by the action of a torus.

Cox rings are finitely generated $k$-algebras in several other cases, including del Pezzo surfaces [Batyrev and Popov 2004], rational surfaces with big anticanonical divisor [Testa et al. 2009], blow-ups of $\mathbb{P}^{n}$ at points lying on a rational normal curve [Castravet and Tevelev 2006] and wonderful varieties [Brion 2007]. All these varieties are examples of Mori dream spaces [Hu and Keel 2000], and for this

MSC2000: $14 \mathrm{~J} 26$.

Keywords: Cox rings, total coordinate rings, del Pezzo surfaces.

The first author was partially supported by Jacobs University Bremen, DFG grant STO-299/4-1 and EPSRC grant number EP/F060661/1; the third author is partially supported by NSF grant DMS0802851 . 
class the Cox ring of $X$ captures much of the birational geometry of the variety. For example, the effective and nef cones of $X$ are finitely generated polyhedral cones and there are only finitely many varieties isomorphic to $X$ in codimension one, satisfying certain mild restrictions. To add to the long list of consequences of the landmark paper [Birkar et al. 2008], we note that log Fano varieties are also Mori dream spaces.

Colliot-Thélène and Sansuc introduced universal torsors to aid the study of the Hasse principle and weak approximation on an algebraic variety $X$ over a number field [Colliot-Thélène and Sansuc 1980; 1987]; see also [Colliot-Thélène et al. 1984]. If the Cox ring of $X$ is finitely generated, then the universal torsor of $X$ is an open subset of $\operatorname{Spec}(\operatorname{Cox}(X))$, an affine variety for which explicit presentations have been calculated in many cases [Hassett and Tschinkel 2004; Hassett 2004]. Starting with Salberger [1998], universal torsors have been successfully applied to the problem of counting points of bounded height on many classes of varieties. The expository article [Peyre 2004] has a very readable account of the ideas involved. The explicit descriptions of universal torsors via Cox rings have led to explicit examples of generalized del Pezzo surfaces that satisfy Manin's conjectures for points of bounded height [de la Bretèche and Browning 2007; de la Bretèche et al. 2007].

Batyrev and Popov [2004] systematically study the Cox rings of del Pezzo surfaces $X / k$. They show that $\operatorname{Cox}(X)$ is a finitely generated $k$-algebra; moreover, if $\operatorname{deg}(X) \leq 6$ then $\operatorname{Cox}(X)$ is generated by sections whose classes have anticanonical degree one [Batyrev and Popov 2004, Theorem 3.2]. Let $\mathscr{G}$ denote a minimal set of homogeneous generators of $\operatorname{Cox}(X)$, and denote by $k[G]$ the polynomial ring whose variables are indexed by the elements of $\mathscr{G}$. As a result, $\operatorname{Cox}(X)$ is a quotient of $k[\varphi]$ :

$$
\operatorname{Cox}(X) \cong k[\varphi] / I_{X} .
$$

Batyrev and Popov provided a conjectural description of the generators of $I_{X}$.

Conjecture 1.1 [Batyrev and Popov 2004]. Let X be a del Pezzo surface. The ideal $I_{X}$ is generated by quadrics.

Quadratic relations have a clear geometric meaning: linear systems associated to degree two nef divisors on $X$ have many more reducible elements than their dimension; thus there are linear dependence relations among degree two monomials in $k[G]$. All quadratic relations arise in this way (see page 758).

Remark 1.2. Del Pezzo surfaces of degree at least six are toric varieties and hence their Cox rings are polynomial rings. The Cox ring of a del Pezzo surface of degree five is the homogeneous coordinate ring of the Grassmannian $\operatorname{Gr}(2,5)$ [Batyrev and Popov 2004]. 
Several partial results are known about this conjecture. Stillman, Testa, and Velasco established it for del Pezzo surfaces of degree four, and general del Pezzo surfaces of degree three [Stillman et al. 2007]. The conjecture was proved in [Laface and Velasco 2009] for surfaces of degree at least two. Recently, Serganova and Skorobogatov [2007; 2008] established Conjecture 1.1 up to radical for surfaces of degree at least two, using representation theoretic methods, (see also [Derenthal 2007] for related work). In all cases, the conjecture for degree one surfaces eluded proof. The purpose of this paper is to fill in this gap for surfaces defined over a field of characteristic different from two, as well as for certain general surfaces in characteristic two that we call sweeping (see Definition 4.3).

Theorem 1.3. Let $X$ be a del Pezzo surface of degree one; if the characteristic of $k$ is two, then assume that $X$ is sweeping. The ideal $I_{X}$ is generated by quadrics.

Remark 1.4. Our method of proof is cohomological in nature and relies mainly on the Kawamata-Viehweg vanishing theorem. By [Terakawa 1998] and [Xie 2008], this theorem is independent of the characteristic of $k$ for rational surfaces.

The argument we give works for del Pezzo surfaces of every degree. We include a short proof of the conjecture for del Pezzo surfaces of degree at least two in Section 10. An alternative proof of Conjecture 1.1 for general del Pezzo surfaces in characteristic zero, obtained independently by computational means, appears in a paper by Sturmfels and Xu [2008]. They apply the theory of sagbi bases to construct an initial toric ideal of $I_{X}$ which is generated by quadrics. Geometrically this corresponds to a degeneration of the universal torsor on $\mathrm{X}$ to a suitable toric variety.

In order to prove Theorem 1.3, we modify the approach taken in [Laface and Velasco 2009]. We use a complex of vector spaces whose homology determines part of the structure of the minimal free $\operatorname{Pic}(X)$-graded resolution of $\operatorname{Cox}(X)$ over a polynomial ring. We show that sufficiently many Betti numbers of this minimal free resolution vanish to establish the theorem. We hope that similar techniques can be applied to obtain presentations of Cox rings of other classes of varieties, for example singular del Pezzo surfaces, blow-ups of $\mathbb{P}^{n}$ at points lying on the rational normal curve of degree $n$ and $\bar{M}_{0, n}$.

The paper is organized as follows. In Section 2 we define del Pezzo surfaces, fix presentations for their Cox rings and establish notation for the rest of the paper. In Section 3 we study the nef cone of del Pezzo surfaces and prove some geometric results. In Section 4 we analyze low-degree linear systems on del Pezzo surfaces of degree at most two. Our proof of Theorem 1.3 uses [Batyrev and Popov 2004, Proposition 3.4]. However, the proof given by those authors for their proposition has a gap: it applies only to general del Pezzo surfaces of degree one in characteristic not two [Popov 2004]. Thus we prove Proposition 4.4 in order to establish 
Theorem 1.3 for all del Pezzo surfaces of degree one in characteristic not two. In Section 5 we review the approach of [Laface and Velasco 2009] to study the ideal of relations of finitely generated Cox rings, adapting it to the case of degree one del Pezzo surfaces. In Section 6 we define the notions of capturability of a divisor and stopping criterion, and prove Theorem 6.7, the main ingredients in our proof of the Batyrev-Popov conjecture. In Section 7 we show the capturability of most divisors on del Pezzo surfaces of degree one. We then handle the remaining cases for del Pezzo surfaces of degree one (Sections 8, 9) and del Pezzo surfaces of higher degree (Section 10). In Section 11 we finish the proof of the BatyrevPopov conjecture and give the first multigraded Betti numbers of the Cox rings of del Pezzo surfaces.

\section{Notation and background on del Pezzo surfaces}

We briefly review some facts about del Pezzo surfaces and establish much of the paper's notation along the way.

Definition 2.1. A del Pezzo surface $X$ is a smooth, projective surface over $k$ whose anticanonical divisor $-K_{X}$ is ample. The degree of $X$ is the integer $\left(K_{X}\right)^{2}$.

Picard groups and Cox rings. A del Pezzo surface $X$ not isomorphic to $\mathbb{P}^{1} \times \mathbb{P}^{1}$ is isomorphic to a blow-up of $\mathbb{P}^{2}$ centered at $r \leq 8$ points in general position: this means no three points on a line, no six on a conic and no eight on a singular cubic with a singularity at one of the points. Let $L$ be the inverse image of a line in $\mathbb{P}^{2}$ and let $E_{1}, \ldots, E_{r}$ be the exceptional divisors corresponding to the blown-up points. Then $\left(L, E_{1}, \ldots, E_{r}\right)$ is a basis for $\operatorname{Pic}(X)$, and

$$
\operatorname{Cox}(X):=\bigoplus_{\left(m_{0}, \ldots, m_{r}\right) \in \mathbb{Z}^{r+1}} \mathrm{H}^{0}\left(X, \mathrm{O}_{X}\left(m_{0} L+m_{1} E_{1}+\cdots+m_{r} E_{r}\right)\right) .
$$

If $X$ has degree one, then with the above choice of basis, the classes in $\operatorname{Pic}(X)$ of the 240 exceptional curves are given in Table 1 [Manin 1986].

\begin{tabular}{|c|l|}
\hline \# of curves & Picard degree (up to a permutation of $E_{1}, \ldots, E_{8}$ ) \\
\hline 8 & $E_{1}$ \\
28 & $L-E_{1}-E_{2}$ \\
56 & $2 L-E_{1}-E_{2}-E_{3}-E_{4}-E_{5}$ \\
56 & $3 L-2 E_{1}-E_{2}-E_{3}-E_{4}-E_{5}-E_{6}-E_{7}$ \\
56 & $4 L-2 E_{1}-2 E_{2}-2 E_{3}-E_{4}-E_{5}-E_{6}-E_{7}-E_{8}$ \\
28 & $5 L-2 E_{1}-2 E_{2}-2 E_{3}-2 E_{4}-2 E_{5}-2 E_{6}-E_{7}-E_{8}$ \\
8 & $6 L-3 E_{1}-2 E_{2}-2 E_{3}-2 E_{4}-2 E_{5}-2 E_{6}-2 E_{7}-2 E_{8}$ \\
\hline
\end{tabular}

Table 1. Exceptional curves on $X$. 
For a del Pezzo surface $X$ of any degree, we let $\mathscr{b}$ denote the set of exceptional curves on $X$. If $X$ has degree one, we let

$$
\mathscr{G}:=\mathscr{C} \cup\left\{K_{1}, K_{2}\right\}
$$

where $K_{1}, K_{2} \in\left|-K_{X}\right|$; if $X$ has degree at least two, then we let

$$
\mathscr{G}:=\mathscr{C} \text {. }
$$

In all cases, we write $k[G]$ for the polynomial ring whose variables are indexed by the elements of $\mathscr{G}$. By [Batyrev and Popov 2004, Theorem 3.2], we know that $\operatorname{Cox}(X)$ is a finitely generated $k$-algebra, generated by sections whose divisor classes have anticanonical degree one. For all $G \in \mathscr{G}$, we let $g \in \mathrm{H}^{0}\left(X, O_{X}(G)\right)$ be a nonzero element. We typically use uppercase letters for divisors on $X$ and we denote the generator of $k[G]$ associated to an exceptional curve $E$ by the corresponding lowercase letter $e$. If $X$ has degree one, then we denote the generators of $k[G]$ corresponding to $K_{1}$ and $K_{2}$ by $k_{1}$ and $k_{2}$, respectively. Furthermore, given any exceptional curve $E \in \mathscr{C}$, we let $E^{\prime}$ denote the unique exceptional curve whose divisor class is $-2 K_{X}-E$, and we denote accordingly the generator corresponding to $E^{\prime}$ by $e^{\prime}$.

There is a surjective morphism

$$
k[G] \rightarrow \operatorname{Cox}(X)
$$

that maps $g$ to $g$. We denote the kernel of this map by $I_{X}$.

For any divisor $D$ on $X$, any integer $n$ and any $\operatorname{Pic}(X)$-graded ideal $J \subset k[G]$, denote by $J_{D}$ the vector space of homogeneous elements of $\operatorname{Pic}(X)$-degree $D$, by $J_{n}$ the vector space of the homogeneous elements of anticanonical degree $n$ and call them, respectively, the degree $D$ part of $J$ and the degree $n$ part of $J$.

Finally, let $J_{X}$ be the ideal generated by $\left(I_{X}\right)_{2}$; since $J_{X} \subset I_{X}$, there is a surjection

$$
\left(k[\mathscr{G}] / J_{X}\right)_{D} \rightarrow\left(k[\mathscr{G}] / I_{X}\right)_{D} .
$$

This map plays a role in Section 8.

\section{Remarks on the nef cone of del Pezzo surfaces}

In this section we collect basic results on del Pezzo surfaces that we use in the paper. The following result is well known [Debarre 2001, page 148, 6.5].

Proposition 3.1. Let $X$ be a del Pezzo surface of degree $d \leq 7$. A divisor class $C \in \operatorname{Pic}(X)$ is nef (respectively ample) if and only if $C \cdot E \geq 0$ (respectively $C \cdot E>0$ ) for all $(-1)$-curves $E \subset X$.

Definition 3.2. Let $X$ be a del Pezzo surface. The minimal ample divisor on $X$ is the ample divisor $A_{X}$ defined in the following table: 


\begin{tabular}{|c|c|c|}
\hline$X$ & $A_{X}$ & $\left(K_{X}\right)^{2}$ \\
\hline $\mathbb{P}^{2}$ & $-\frac{1}{3} K_{X}$ & 9 \\
$\mathbb{P}^{1} \times \mathbb{P}^{1}$ & $-\frac{1}{2} K_{X}$ & 8 \\
$B l_{p}\left(\mathbb{P}^{2}\right)$ & $2 L-E$ & 8 \\
$B l_{p_{1}, \ldots, p_{r}}\left(\mathbb{P}^{2}\right)$ & $-K_{X}$ & $9-r$ \\
\hline
\end{tabular}

We also extend the list to include $X=\mathbb{P}^{1}$ and we define the minimal ample divisor on $\mathbb{P}^{1}$ to be the class of a point. To simplify the notation, if $b: X \rightarrow Y$ is a morphism, then we may denote $b^{*}\left(A_{Y}\right)$ by $A_{Y}$. Minimal ample divisors allow us to give a geometric description of nef divisors.

Corollary 3.3. Let $X_{r}$ be a del Pezzo surface of degree $9-r$. Let $D \in \operatorname{Pic}\left(X_{r}\right)$ be a nef divisor. Then we can find nonnegative integers $n_{0}, n_{1}, \ldots, n_{r}$ and a sequence of morphisms $X_{r} \longrightarrow X_{r-1} \longrightarrow \cdots \longrightarrow X_{1} \longrightarrow X_{0}$ such that

- each morphism is the contraction of a (-1)-curve, except for $X_{1} \rightarrow X_{0}$, which is allowed to be a conic bundle; and

- D can be expressed as

$$
D=n_{r} A_{X_{r}}+n_{r-1} A_{X_{r-1}}+\cdots+n_{0} A_{X_{0}} .
$$

Proof. We proceed by induction on $r$, the cases $r \leq 1$ being immediate. Suppose that $r \geq 2$ and let $n:=\min \{L \cdot D \mid L \subset X$ is a $(-1)$-curve $\}$. By assumption we have $n \geq 0$. Let $\bar{D}:=D+n K_{X_{r}}$; note that $\bar{D}$ is nef by Proposition 3.1. Choose a (-1)-curve $L_{0} \subset X$ such that $\bar{D} \cdot L_{0}=0$. Thus $\bar{D}$ is the pull-back of a nef divisor on the del Pezzo surface $X_{r-1}$ obtained by contracting $L_{0}$. The result follows by the inductive hypothesis.

Observe that the integer $n_{r}$ in the statement of the corollary is the nef threshold ([Reid 1997, page 126]) of the divisor $D$ with respect to the minimal ample divisor of $X_{r}$. The minimal ample divisor is minimal in the sense that for every ample divisor $A$ on $X$, the divisor $A-A_{X}$ is nef: this follows from Corollary 3.3.

By [Kollár 1996, Proposition III.3.4] and Corollary 3.3 we deduce that every nef divisor $N$ on a del Pezzo surface is effective and that $|2 N|$ is base-point free. Moreover, if $N$ is a nef nonbig divisor, then $N$ is a multiple of a conic bundle.

Let $X \rightarrow Y$ be a morphism with connected fibers and let $A$ be the pull-back to $X$ of the minimal ample divisor on $Y$. If $Y \simeq \mathbb{P}^{1}$, then we call $A$ a conic. If $Y \simeq \mathbb{P}^{2}$, then we call $A$ a twisted cubic, by analogy with the case of cubic surfaces. Finally, if $Y$ is a del Pezzo surface of degree $d$, then we call $A$ an anticanonical divisor of degree $d$ in $X$.

We summarize and systematize the previous discussion in the following lemma. 
Lemma 3.4. Let $X$ be a del Pezzo surface. The cone of nef divisors on $X$ is generated by the following divisors:

(1) the conics;

(2) the twisted cubics;

(3) the anticanonical divisors of degree $d \leq 3$ in $X$.

In particular, if $N$ is any nonzero nef divisor on $X$, then we may find $r:=8-\operatorname{deg}(X)$ distinct exceptional curves $E_{1}, E_{2}, \ldots, E_{r}$ on $X$ such that $N-E_{1}, \ldots, N-E_{r}$ are either nef or the sum of a nef divisor and an exceptional curve, unless $\operatorname{deg}(X)=1$ and $N=-K_{X}$.

Proof. The divisors in the list are clearly nef. Conversely, if $D$ is any nef divisor, then either $D$ is a multiple of a conic, and we are done, or a positive multiple of $D$ induces a morphism with connected fibers $X \rightarrow Y$. It is clear that if $A$ is the pull-back to $X$ of the minimal ample divisor on $Y$, then $D-A$ is nef. By induction on $n:=-K_{X} \cdot D$, we therefore reduce to showing that the anticanonical divisors of degree $d \geq 4$ in $X$ are nonnegative linear combinations of the divisors listed. This is immediate.

For the second statement, note that it suffices to check it for the divisors in the list, and for $-2 K_{X}$ if $\operatorname{deg}(X)=1$, where the result is easy to verify.

The following lemma will be used in the proof of Lemma 7.7.

Lemma 3.5. Let $X$ be a del Pezzo surface, let $b: X \rightarrow \mathbb{P}^{1}$ be a conic bundle with fiber class $Q$ and let $C$ be an exceptional curve such that $C \cdot Q=2$. There are exactly five reducible fibers of $b$ such that $C$ intersects both components. In particular, if $\operatorname{deg}(X)=1$, then there are two reducible fibers of $b$ such that $C$ is disjoint from one of the two components in each fiber.

Remark 3.6. If $\operatorname{deg}(X) \geq 4$, then there are no exceptional curves $C$ such that $C \cdot Q=2$, and hence the lemma applies nontrivially only to the cases $\operatorname{deg}(X) \leq 3$.

Proof. Let $S$ and $T$ be the components of a reducible fiber of $b$. Since $C \cdot Q=2$, there are only two possibilities for the intersection numbers $C \cdot S$ and $C \cdot T$ : they are either both equal to one, or one of them is zero and the other is two. Suppose that there are $k$ reducible fibers of $b$ such that the curve $E$ intersects both components. Thus contracting all the components in fibers of $b$ disjoint from $E$ and one component in each of the remaining reducible fibers, we obtain a relatively minimal ruled surface $b^{\prime}: X^{\prime} \rightarrow \mathbb{P}^{1}$, together with a smooth rational curve $C^{\prime}$ in $X^{\prime}$, the image of $C$, having anticanonical degree $k+1$, square $k-1$ and intersection number two with a fiber of $b^{\prime}$. Since $X^{\prime}$ is isomorphic to either $\mathbb{P}^{1} \times \mathbb{P}^{1}$ or $B l_{p}\left(\mathbb{P}^{2}\right)$, a direct calculation shows that this is only possible if $X^{\prime} \simeq \mathbb{P}^{1} \times \mathbb{P}^{1}$ and $k=5$.

The last statement follows from the fact that any conic bundle structure on $X$ contains exactly $8-\operatorname{deg}(X)$ reducible fibers. 


\section{Low-degree linear systems}

The lemmas in this section determine subsets of monomials that span $\operatorname{Cox}(X)_{D}$ when $(\operatorname{deg}(X), D) \in\left\{\left(2,-K_{X}\right),\left(1,-2 K_{X}\right)\right\}$.

Lemma 4.1. Let $X$ be a del Pezzo surface of degree two. The linear system $\left|-K_{X}\right|$ is spanned by any five of its reducible elements.

Proof. The linear system $\left|-K_{X}\right|$ defines a separable morphism of degree two $\varphi: X \rightarrow \mathbb{P}^{2}$ such that every pair of exceptional curves $E, E^{\prime}$ on $X$ with $E+E^{\prime}=$ $-K_{X}$ maps under $\varphi$ to the same line in $\mathbb{P}^{2}$. To prove the result it suffices to show that any five of these lines have no base-point. Suppose that $p \in \mathbb{P}^{2}$ is contained in $k_{p} \geq 4$ such lines; let $q \in X$ be a point in the inverse image of $p$. By construction, the point $q$ is contained in $k_{p}$ exceptional curves. Let $E$ be an exceptional curve containing $q$ and let $E^{\prime}$ be the exceptional curve such that $E+E^{\prime}=-K_{X}$. The curve $E^{\prime}$ is disjoint from the exceptional curves $F$ such that $F \cdot E=1$, since $F \cdot E^{\prime}=0$. Thus contracting $E^{\prime}$ we obtain a del Pezzo surface $Y$ of degree three with a point contained in at least $k_{p}-1$ exceptional curves. The anticanonical linear system embeds $Y$ as a smooth cubic surface in $\mathbb{P}^{3}$, and exceptional curves through a point $y \in Y$ are lines contained in the tangent plane to $Y$ at $y$. This implies that $k_{p}-1 \leq 3$, and the result follows.

To study the case of degree one del Pezzo surfaces we begin with a lemma.

Lemma 4.2. Let $R \subset \mathbb{P}^{3}$ be a curve, let $r \in R$ be a smooth point and let $p \in \mathbb{P}^{3}$ be a closed point different from $r$. Let $H$ be a plane through $p$ intersecting $R$ at $r$ with order of contact $m \geq 2$. Let $\pi_{R}: R \rightarrow \mathbb{P}^{2}$ be the projection away from $p$, let $\bar{R}=\pi_{R}(R)$, and let $\gamma: R \rightarrow\left(\mathbb{P}^{2}\right)^{\vee}$ be the composition of $\pi_{R}$ and the Gauss map of $\bar{R}$, i.e., the map sending a general point $q \in R$ to the tangent line to $\bar{R}$ at $\pi_{R}(q)$. Then the map $\gamma$ is defined at $r$ and one of the following occurs:

(1) the tangent line to $R$ at $r$ contains $p$, or

(2) the length of the localization at $r$ of the fiber of $\gamma$ at $\gamma(r)$ equals $m-1$ if $\operatorname{char}(k) \nmid m$ and it equals $m$ if $\operatorname{char}(k) \mid m$.

Proof. The rational map $\gamma$ is defined at $r$ since $r \in R$ is a smooth point and the range of $\gamma$ is projective. Choose homogeneous coordinates $X_{0}, X_{1}, X_{2}, X_{3}$ on $\mathbb{P}^{3}$ so that $p=[1,0,0,1]$ and $r=[0,0,0,1]$. In these coordinates $H$ is defined by the linear form $A X_{1}+B X_{2}$, for some $A, B \in k$. In the affine coordinates $\left(x_{0}=X_{0} / X_{3}, x_{1}=X_{1} / X_{3}, x_{2}=X_{2} / X_{3}\right)$ the curve $R$ is defined near the origin $r$ by a complete intersection $\left(f_{1}, f_{2}\right)$ and thus its embedded tangent space is the kernel of the matrix $D F(r)=\left(\left(\partial f_{i} / \partial x_{j}\right)(r)\right)$ with $i \in\{1,2\}$ and $j \in\{0,1,2\}$. Therefore either the first column of $D F(r)$ vanishes - that is, $\partial f_{1} / \partial x_{0}$ and $\partial f_{2} / \partial x_{0}$ vanish at $r$-and the tangent line to $R$ through $r$ contains $p$, or some $2 \times 2$ minor of 
$D F(r)$ containing the first column is nonzero, since otherwise all $2 \times 2$ minors of $D F(r)$ vanish, contradicting the assumption that $R$ is nonsingular at $r$. In the latter case, the completion of the local ring of $R$ at $r$ is isomorphic to $k \llbracket t \rrbracket$ via a parametrization in formal power series of the form $\left(x_{0}(t), x_{1}(t), t\right)$. Since $H$ has order of contact $m$ at $r$, the power series $A x_{1}(t)+B t$ vanishes to order $m$ so $A \neq 0$ and we have

$$
x_{1}(t)=-\frac{B}{A} t+c_{m} t^{m}+\text { (higher order terms) }
$$

with $c_{m} \neq 0$. The equation of the tangent line to $\bar{R}$ at $\pi_{R}\left(x_{0}(t), x_{1}(t), t, 1\right)$ is

$$
-\left(X_{1}-x_{1}(t)\right)+\left(X_{2}-t\right) x_{1}^{\prime}(t)=0,
$$

so the morphism $\gamma$ is given by

$$
\left(x_{0}(t), x_{1}(t), t, 1\right) \longmapsto\left[-1, x_{1}^{\prime}(t), x_{1}(t)-t x_{1}^{\prime}(t)\right] .
$$

The localization at $r$ of the fiber of $\gamma$ at $\gamma(r)$ is thus given by the ideal

$$
I=\left(A x_{1}^{\prime}(t)+B, x_{1}(t)-t x_{1}^{\prime}(t)\right) .
$$

Since we have

$$
\begin{gathered}
A x_{1}^{\prime}(t)+B=A c_{m} m t^{m-1}+\text { (higher order terms), } \\
x_{1}(t)-t x_{1}^{\prime}(y)=c_{m}(1-m) t^{m}+\text { (higher order terms), }
\end{gathered}
$$

it follows that either $\operatorname{char}(k) \nmid m$ and $I=\left(t^{m-1}\right)$, or $\operatorname{char}(k) \mid m$ and $I=\left(t^{m}\right)$ as we wanted to show.

Let $X$ be a del Pezzo surface of degree one. The linear system $\left|-2 K_{X}\right|$ is basepoint free and the image of the associated morphism $\kappa: X \rightarrow\left|-2 K_{X}\right|^{*} \simeq \mathbb{P}^{3}$ is a cone $W$ over a smooth conic; the morphism $\kappa$ is a separable double cover of $W$ branched at the cone vertex $w \in W$ and along a divisor $R \subset W$. We call tritangent planes of $X$ the elements of $\left|-2 K_{X}\right|$ supported on exceptional curves, and think of them as planes in $\left|-2 K_{X}\right|^{*}=\mathbb{P}^{3}$. There are 120 such planes and they do not contain the vertex $w$ of the cone, since the planes containing $w$ correspond to elements of $\left|-2 K_{X}\right|$ supported on the sum of two effective anticanonical divisors (see [Kollár 1996, Section III.3] and [Manin 1986, Chapter IV] for details).

Definition 4.3. A del Pezzo surface $X$ of degree one over a field $k$ is sweeping if any 119 tritangent planes of $X$ span $\left|-2 K_{X}\right|$.

Our goal in the remainder of this section is to show that every del Pezzo surface of degree one in characteristic not two is sweeping; if the characteristic is two, then we only show that a general del Pezzo surface of degree one is sweeping. In the following sections we prove that if $X$ is a sweeping del Pezzo surface of degree one, then Conjecture 1.1 holds for $X$. 
From here through page 740 we assume that $k$ does not have characteristic 2 . Then the curve $R$ is smooth and it is the complete intersection of $W$ with a cubic surface. Hence $R$ is a canonical curve of genus four and degree six admitting a unique morphism of degree three to $\mathbb{P}^{1}$ (up to changes of coordinates on $\mathbb{P}^{1}$ ) obtained by projecting away from $w$. The tritangent planes to $R$ are planes in $\mathbb{P}^{3}$ not containing $w$ whose intersection with $R$ is twice an effective divisor.

Proposition 4.4. If $X$ is a del Pezzo surface of degree one over a field $k$ of characteristic different from two, then $X$ is sweeping. More precisely, any 113 of the tritangent planes of $X$ span $\left|-2 K_{X}\right|$.

Proof. Let $p \in \mathbb{P}^{3}$ be a closed point. We say that a tritangent plane $H$ containing $p$ is $p$-regular (resp. $p$-singular) if $p$ does not belong to (resp. $p$ belongs to) a tangent line to $R$ at some point in $H \cap R$; denote by $k_{p}$ the number of tritangent planes containing $p$ and by $k_{p}^{r}$ (resp. $k_{p}^{s}$ ) the number of $p$-regular (resp. $p$-singular) tritangent planes. Suppose that the point $p$ belongs to $R$; then there are no $p$ regular tritangent planes, since every tritangent plane containing $p$ contains also the tangent line to $R$ at $p$. Thus if $q$ is any point on the tangent line to $R$ at $p$, we have $k_{p} \leq k_{q}^{s} \leq k_{q}$, so it suffices to prove the proposition assuming that $p \in \mathbb{P}^{3}$ is a closed point not in $R$. Therefore let $p \in \mathbb{P}^{3} \backslash R$ be a closed point; projecting away from $p$ we obtain a morphism $\pi_{R}: R \rightarrow \mathbb{P}^{2}$; let $\bar{R}=\pi_{R}(R)$. Let $\bar{\gamma}: \bar{R} \rightarrow\left(\mathbb{P}^{2}\right)^{\vee}$ be the Gauss map, let $\gamma: R \rightarrow\left(\mathbb{P}^{2}\right)^{\vee}$ be the unique morphism that extends the composition $\bar{\gamma} \circ\left(\pi_{R}\right)$, and let $\check{R}=\gamma(R)$. The morphism $\gamma$ factors as $R \stackrel{g}{\rightarrow} N \stackrel{v}{\rightarrow} \check{R}$, where $v: N \rightarrow \check{R}$ is the normalization of $\check{R}$. We summarize these definitions in a commutative diagram:

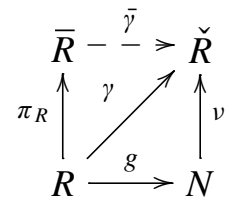

The argument consists in identifying the contributions of the tritangent planes through $p$ in terms of combinatorial invariants of Diagram 1. More precisely, the $p$-singular tritangent planes correspond (at most 7:1) to the points of $\bar{R}$ where the morphism $\pi_{R}$ is ramified; the $p$-regular tritangent planes correspond to points of $\check{R}$ where (the separable part of) $\gamma$ is ramified.

We now estimate the number $k_{p}^{s}$ of $p$-singular tritangent planes.

Lemma 4.5. As above, let $R$ be the ramification divisor of $\kappa$.

(1) If $\ell \subset \mathbb{P}^{3}$ is a tangent line to $R$, then there are at most 7 tritangent planes containing $\ell$;

(2) Let $p \in \mathbb{P}^{3}$ be a closed point not in $R$. There are at most $42 p$-singular tritangent planes. 
Proof of Lemma 4.5. (1) Let $r \in R$ be a point such that $\ell$ is tangent to $R$ at $r$ and let $\alpha: R \rightarrow \mathbb{P}^{1}$ be the morphism obtained by considering the pencil of planes in $\mathbb{P}^{3}$ containing $\ell$. The morphism $\alpha$ has degree at most four since every plane containing $\ell$ is tangent to $R$ at $r$ and hence intersects $R$ at $r$ with multiplicity at least two. If $\alpha$ were not separable, then the characteristic of $k$ would be three (recall that we assume $\operatorname{char}(k) \neq 2$ ), $\alpha$ would be the Frobenius morphism, and the curve $R$ would be rational, which is not the case. We deduce that $\alpha$ is separable; if $\alpha$ has degree three, then $\ell$ contains the vertex $w$ of the cone $W$ and in this case no tritangent plane contains $\ell$. Moreover, $\alpha$ cannot have degree two since $R$ is not hyperelliptic. Thus $\alpha$ is separable and we reduce to the case in which the degree of $\alpha$ is four. If $H$ is a tritangent plane to $R$ containing $\ell$, then we have $R \cap H=2((r)+(p)+(q))$, and hence the contribution of $H$ to the ramification divisor of $\alpha$ is at least two. From the Hurwitz formula we deduce that the ramification divisor has degree 14 and $r$ is contained in at most $14 / 2=7$ tritangent planes.

(2) First, we reduce to the case that the morphism $\pi_{R}$ has degree at most two (and in particular it is separable). Indeed, the degree of $\pi_{R}$ divides six, and since $R$ is not contained in a plane, the image $\bar{R}$ cannot be a line; therefore it suffices to analyze the cases in which the degree of $\pi_{R}$ equals three. If $\pi_{R}$ were not separable of degree three, then it would be purely inseparable and $\bar{R}$ would have degree two but geometric genus four, which is not possible. If $\pi_{R}$ is separable of degree three, then $p$ is the vertex $w$ of the cone $W$ and hence $k_{p}^{s}=0$, since there are no tritangent planes through $w$. This completes the reduction. Note that if the degree of $\pi_{R}$ equals two, then the image of $\pi_{R}$ is a smooth plane cubic, since $R$ is not hyperelliptic.

Second, by [Hartshorne 1977, Proposition IV.3.4], the morphism $\pi_{R}$ ramifies at every tangent line to $R$ through $p$.

Finally, the image of a ramification point of $\pi_{R}$ is either a singular point of $\bar{R}$ or a ramification point of the morphism induced by $\pi_{R}$ to the normalization of $\bar{R}$. Using the Hurwitz formula, the formula for the arithmetic genus of a plane curve, and the equality $\operatorname{deg}(\bar{R}) \cdot \operatorname{deg}\left(\pi_{R}\right)=6$, we deduce that the number of points in $\mathbb{P}^{2}$ corresponding to ramification points of $\pi_{R}$ is at most six. Thus there are at most six lines in $\mathbb{P}^{3}$ containing $p$ such that every $p$-singular tritangent plane contains one of these lines. We conclude using Lemma 4.5(1).

By Lemma 4.5(2) we have $k_{p}^{s} \leq 42$. Thus to prove the proposition, it suffices to show that $k_{p}^{r} \leq 70$.

Define the torsion sheaf $\Delta$ on $\check{R}$ by the exact sequence

$$
0 \rightarrow \mathrm{O}_{\check{R}} \rightarrow v_{*} \mathrm{O}_{N} \rightarrow \Delta \rightarrow 0 .
$$

From the long exact cohomology sequence, we deduce that

$$
h^{1}\left(N, \mathscr{O}_{N}\right)+h^{0}(\Delta)=h^{1}\left(\check{R}, \mathscr{O}_{\check{R}}\right)
$$


Applying the Hurwitz formula to the composition of $\pi_{R}$ with a projection of $\bar{R}$ from a general point of $\mathbb{P}^{2}$, we obtain $\operatorname{deg}(\check{R}) \leq 18$, and hence $h^{1}\left(\check{R}, \mathscr{O}_{\check{R}}\right) \leq 136$.

For each tritangent plane $H$ through $p$ denote by $\ell_{H} \in \check{R} \subset\left(\mathbb{P}^{2}\right)^{\vee}$ the point corresponding to the image in $\mathbb{P}^{2}$ of $H$ under the projection away from $p$. We want to estimate the contribution of the point $\ell_{H} \in \check{R}$ to $h^{0}(\Delta)$. Note that this contribution is at least length $\left(\mathcal{O}_{v^{-1}\left(\ell_{H}\right)}\right)-1$, and that

$$
\text { length }\left(\mathscr{O}_{v^{-1}\left(\ell_{H}\right)}\right) \cdot \operatorname{deg}(g)=\operatorname{length}\left(\mathscr{O}_{\gamma^{-1}\left(\ell_{H}\right)}\right) \text {. }
$$

Factor $g$ as the composition of a power of the Frobenius morphism, followed by a separable morphism $g_{s}$. Let $d_{i}$ be the inseparable degree of $g$ and $d_{s}=\operatorname{deg}\left(g_{s}\right)$ be the separable degree of $g$, let also $\breve{d}$ denote the degree of $\check{R}$; recall that we have $d_{i} d_{s} \check{d} \leq 18$.

Suppose first that $d_{i} d_{s}=1$. By Lemma 4.2 and (4-4) each $p$-regular tritangent plane contributes at least 2 to $h^{0}(\Delta)$; hence we have $h^{0}(\Delta) \geq 2 k_{p}^{r}$, and we conclude that $k_{p}^{r} \leq 68$.

Suppose now that $d_{i} d_{s}>1$ and hence $\breve{d} \leq 9$. By the Hurwitz formula, the degree of the ramification divisor of the morphism $g_{s}$ is at most $6+2 \operatorname{deg}\left(g_{s}\right) \leq 6+36=42$. The curve $\check{R}$ has degree at most nine, and hence it has at most 28 singular points.

Let $U \subset N \times \mathbb{P}^{3}$ be the universal family of planes through $p$ tangent to $R$ and let $Z:=(U \cap N \times R)$. Thus $Z$ defines a family of closed subschemes of $R \subset \mathbb{P}^{3}$ of dimension zero and degree six. The geometric generic fiber of $Z \rightarrow N$ therefore determines a partition of 6 that we call the generic splitting type. Alternatively, if $H$ is a plane through $p$ tangent to $R$, then $H \cap R$ determines an effective divisor of degree six on $R$; if $H$ is general with the required properties, then the multiplicities of the geometric points of $H \cap R$ are the generic splitting type.

By [Hefez and Kleiman 1985, Theorem 3.5] and [Kaji 1992, page 529], the generic splitting type of $\gamma$ is of the form $(a, \ldots, a, 1, \ldots, 1)$, where either $a=2$ and the morphism is separable or $a$ is the inseparable degree of the rational map $\bar{R} \rightarrow \check{R}$. The possibilities are

$$
(5,1),(4,1,1),(3,3),(3,1,1,1),(2,2,2),(2,2,1,1),(2,1,1,1,1) .
$$

If the generic splitting type is $(2,2,2)$, then the point $p$ is the cone vertex $w$ and in this case $k_{p}=0$. The partition $(2,1,1,1,1)$ corresponds to the case $d_{i} d_{s}=1$ and we already analyzed it. The partitions $(5,1)$ and $(4,1,1)$ can also be excluded, since they imply that $R$ is birational to a plane curve of degree at most $\lfloor 18 / 4\rfloor=4$ and hence cannot have arithmetic genus four.

Examining the remaining generic splitting types and using Lemma 4.2, we deduce that the $p$-regular tritangent planes correspond to ramification points of $g_{s}$. In particular, at most $42+28=70$ such points exist, and the proposition follows. 
Remark 4.6. In characteristic two the curve $R$ above has degree 3, contains the cone vertex $w$, and is not necessarily smooth [Cragnolini and Oliverio 2000]. While we are not able to prove that every del Pezzo surface of degree one in characteristic two is sweeping, we prove it for a general such surface. We write an explicit example of a del Pezzo surface $S$ of degree two defined over $\overline{\mathbb{F}}_{2}$ and five tritangent planes to $S$ no four of which share a common point. Thus $S$ is sweeping; since the property of being sweeping is open, we conclude that a general del Pezzo surface of degree one in characteristic two is sweeping. We found this example using Magma [Bosma et al. 1997].

Let $\mathbb{P}:=\mathbb{P}(1,1,2,3)$ be the weighted projective space over $\overline{\mathbb{F}}_{2}$ with coordinates $x, y, z, w$ and respective weights $1,1,2,3$. Let $S \subset \mathbb{P}$ be the surface defined by

$$
S:\left\{w^{2}+z^{3}+w x z+w y^{3}+x^{6}=0\right\} .
$$

It is immediate to check that $S$ is smooth. Let $t \in \overline{\mathbb{F}}_{2}$ be an element satisfying $t^{2}+t+1=0$; any four of the homogeneous forms

$$
z+x^{2}, \quad z+y^{2}, \quad z+t x^{2}, \quad z+t x^{2}+t^{2} x y+y^{2}, \quad z+t x^{2}+x y+t^{2} y^{2}
$$

are linearly independent. Moreover, they represent tritangent planes since we have

$$
\begin{aligned}
& F\left(x, y, x^{2}, w\right)=w\left(w+x^{3}+y^{3}\right), \\
& F\left(x, y, y^{2}, w\right)=\left(w+\left(x^{3}+t x y^{2}+x^{2} y+t y^{3}\right)\right)\left(w+\left(x^{3}+t^{2} x y^{2}+x^{2} y+t^{2} y^{3}\right)\right), \\
& F\left(x, y, t x^{2}, w\right)=w\left(w+t x^{3}+y^{3}\right), \\
& F\left(x, y, t x^{2}+t^{2} x y+y^{2}, w\right)=\left(w+y\left(x^{2}+x y+t y^{2}\right)\right)\left(w+\left(t x^{3}+t^{2} y^{3}+t x^{2} y\right)\right), \\
& F\left(x, y, t x^{2}+x y+t^{2} y^{2}, w\right)=\left(w+t y\left(x^{2}+t x y+y^{2}\right)\right)\left(w+t\left(x^{3}+t x^{2} y+t y^{3}\right)\right),
\end{aligned}
$$

and we deduce that $S$ is sweeping.

The proof of Corollary 4.7 below given in [Batyrev and Popov 2004, Proposition 3.4] contains a gap pointed out in [Popov 2004]: the original argument implicitly assumes that the characteristic of the base field is not two, and reduces the proof to the fact that $\mathrm{H}^{0}\left(X, O_{X}\left(-2 K_{X}\right)\right)$ is spanned by the sections supported on exceptional curves. Proposition 4.4 fixes this gap.

Corollary 4.7. Let $X$ be a del Pezzo surface of degree one in characteristic not two. If $D \neq-K_{X}$ is an effective divisor, then $\mathrm{H}^{0}\left(X, O_{X}(D)\right)$ is spanned by global sections supported on exceptional curves.

Remark 4.8. A general del Pezzo surface of degree one in characteristic two is sweeping; therefore Corollary 4.7 also holds for such surfaces. 


\section{Betti numbers and the Batyrev-Popov conjecture}

We review the approach of [Laface and Velasco 2009] to study the ideal of relations of Cox rings. As usual, let $X$ be a del Pezzo surface, and let $R=k[G]$. Throughout this section $D$ denotes a divisor on $X$. Since $R$ is positively graded (by anticanonical degree), every finitely generated $\operatorname{Pic}(X)$-graded $R$-module has a unique minimal $\operatorname{Pic}(X)$-graded free resolution. For the module $\operatorname{Cox}(X)=R / I_{X}$ this resolution is of the form

$$
\cdots \rightarrow \bigoplus_{D \in \operatorname{Pic}(X)} R(-D)^{b_{2, D}} \rightarrow \bigoplus_{D \in \operatorname{Pic}(X)} R(-D)^{b_{1, D}} \rightarrow R \rightarrow 0
$$

where the rightmost nonzero map is given by a row matrix whose nonzero entries are a set of minimal generators of the ideal $I_{X}$. Since the differential of the resolution has degree 0 , it follows that $I_{X}$ has exactly $b_{1, D}(\operatorname{Cox}(X))$ minimal generators of Picard degree $D$.

Let $\mathbb{K}$ be the Koszul complex on $\mathscr{G}$. Consider the degree $D$ part of the complex $\operatorname{Cox}(X) \otimes_{R} \mathbb{K}$. Then

$$
\begin{aligned}
\mathrm{H}_{i}\left(\left(\operatorname{Cox}(X) \otimes_{R} \mathbb{K}\right)_{D}\right) & =\left(\mathrm{H}_{i}\left(\operatorname{Cox}(X) \otimes_{R} \mathbb{K}\right)\right)_{D} \\
& =\left(\operatorname{Tor}_{i}^{R}(\operatorname{Cox}(X), k)\right)_{D}=k^{b_{i, D}(\operatorname{Cox}(X))},
\end{aligned}
$$

where the equalities on the last line follow since $\operatorname{Tor}_{i}^{R}(A, B)$ is symmetric in $A$ and $B$ and the Koszul complex is the minimal free resolution of $k$ over $R$. Hence we have the equality

$$
\operatorname{dim}_{k}\left(\mathrm{H}_{i}\left(\left(\operatorname{Cox}(X) \otimes_{R} \mathbb{K}\right)_{D}\right)\right)=b_{i, D}(\operatorname{Cox}(X)) .
$$

Thus, Conjecture 1.1 is equivalent to the statement that $b_{1, D}(\operatorname{Cox}(\mathrm{X}))=0$ for all $D \in \operatorname{Pic}(X)$ with $-K_{X} \cdot D \geq 3$. This is the form of the conjecture that we prove.

Let $X$ be a del Pezzo surface of degree one. To compute the Betti numbers $b_{1, D}(\operatorname{Cox}(X))$, denote by $C_{1}, \ldots, C_{240}$ the exceptional curves of $X$, and let

$$
C_{240+i}:=K_{i}
$$

for $i \in\{1,2\}$. With this notation, the part of the complex relevant to our task is

$$
\bigoplus_{1 \leq i<j \leq 242} \mathrm{H}^{0}\left(X, \mathrm{O}_{X}\left(D-C_{i}-C_{j}\right)\right) \stackrel{d_{2}}{\rightarrow} \bigoplus_{i=1}^{242} \mathrm{H}^{0}\left(X, \mathrm{O}_{X}\left(D-C_{i}\right)\right) \stackrel{d_{1}}{\rightarrow} \mathrm{H}^{0}\left(X, \mathrm{O}_{X}(D)\right),
$$

where $d_{2}$ sends $\sigma_{i j} \in \mathrm{H}^{0}\left(X, \mathrm{O}_{X}\left(D-C_{i}-C_{j}\right)\right)$ to

$$
\left(0, \ldots, 0, \sigma_{i j} c_{j}, 0, \ldots, 0,-\sigma_{i j} c_{i}, 0, \ldots, 0\right)
$$

and $d_{1}$ sends $\sigma_{i} \in \mathrm{H}^{0}\left(X, \mathcal{O}_{X}\left(D-C_{i}\right)\right)$ to $\sigma_{i} c_{i}$. 
A cycle is an element of ker $d_{1}$; a boundary is an element of im $d_{2}$. The support of a cycle $\sigma=\left(\ldots, \sigma_{i}, \ldots\right)$ is

$$
\|\sigma\|=\left\{C_{i}: \sigma_{i} \neq 0\right\}
$$

and the size of the support is the cardinality of $\|\sigma\|$, denoted by $|\sigma|$.

Strategy. In order to show that $b_{1, D}(\operatorname{Cox}(X))=0$ whenever $-K_{X} \cdot D \geq 3$, we split the divisor classes $D$ as follows:

(1) $D$ is ample and has anticanonical degree at least four (Section 7);

(2) $D$ is ample and has anticanonical degree three (Section 8);

(3) $D$ is not ample (Section 9).

The case when $D$ is not ample follows by induction on the degree of the del Pezzo surface, and the ample divisors of anticanonical degree three are dealt with algebraically. In order to show that $b_{1, D}(\operatorname{Cox}(X))=0$ whenever $D$ is ample and $-K_{X} \cdot D \geq 4$ we show that every cycle is a boundary by arguing as follows.

- We describe constructions through which a divisor may be removed from the support of a cycle using boundaries, at the cost of possibly introducing new divisors in the support of the cycle.

- We apply these constructions to all cycles in a systematic way to reduce their support to at most two elements.

We conclude using the following lemma.

Lemma 5.1. Any cycle $\sigma$ with $|\sigma| \leq 2$ is a boundary.

Proof. We deduce from [Elizondo et al. 2004, Theorem 1.1] that the Cox rings of del Pezzo surfaces are unique factorization domains. The statement follows immediately.

\section{Capturability and stopping criteria}

We introduce the following terminology to aide us in our search of ways to reduce the support of a cycle. We keep the notation of the previous section.

Definition 6.1. A capture move for a divisor $D$ is a pair $(\mathscr{S}, C)$, where $\mathscr{Y} \subset \mathscr{G}$, $C \in \mathscr{G} \backslash \mathscr{Y}$ and the map

$$
\bigoplus_{S \in \mathscr{S}} \mathrm{H}^{0}\left(X, \mathrm{O}_{X}(D-S-C)\right) \otimes \mathrm{H}^{0}\left(X, \mathrm{O}_{X}(S)\right) \longrightarrow \mathrm{H}^{0}\left(X, \mathrm{O}_{X}(D-C)\right)
$$

induced by tensor product of sections is surjective. We say that $C$ is the captured curve, and that $C$ is capturable for $D$ by $\mathscr{Y}$. 
Let $T$ be a set; for a direct sum $\bigoplus_{t \in T} V_{t}$ of vector spaces indexed by $T$ and $T^{\prime} \subset T$, we write $\sum_{t \in T^{\prime}} a_{t} \hat{e}_{t}$ for the element $\left(b_{t}\right)_{t \in T}$ of $\bigoplus_{t \in T} V_{t}$ where $b_{t}=a_{t}$ if $t \in T^{\prime}$ and $b_{t}=0$ otherwise. As usual, if $S \in \mathscr{G}$, we denote by $s$ the chosen global section of $\mathrm{O}_{X}(S)$. Let $(\mathscr{Y}, C)$ be a capture move for $D$, let $\sigma_{C} \in \mathrm{H}^{0}\left(X, O_{X}(D-C)\right)$, and let $\sigma \in \bigoplus \mathrm{H}^{0}\left(X, O_{X}\left(D-C_{i}\right)\right)$ be the element corresponding to $\sigma_{C}$. Then we have

$$
\sigma_{C}=\sum_{S \in \mathscr{S}} p_{s} s, \quad p_{s} \in \mathrm{H}^{0}\left(X, \mathrm{O}_{X}(D-S-C)\right),
$$

and thus we obtain

$$
\sigma=\sum_{S \in \mathscr{S}} p_{S} c \hat{e}_{s}+d_{2}\left(\sum_{S \in \mathscr{S}} \varepsilon_{C S} p_{S} \hat{e}_{C+S}\right) \in \bigoplus_{i} \mathrm{H}^{0}\left(X, \hat{O}_{X}\left(D-C_{i}\right)\right)
$$

where $\varepsilon_{C S} \in\{ \pm 1\}$.

Hence, if $\sigma$ is a cycle and there is a capture move $(\mathscr{Y}, C)$, then we can modify $\sigma$ by a boundary so that $C \notin\|\sigma\|$. In this sense we have captured the curve $C$ from the support of $\sigma$. Observe, however, that this modification adds a subset of $\mathscr{S}$ to $\|\sigma\|$, so a priori the size of the support may not have decreased. We need to find and apply capture moves in an organized way to ensure that we are genuinely decreasing the size of the support of a cycle.

Finally note that if $(\mathscr{Y}, C)$ is a capture move and $\mathscr{Y}^{\prime} \supset \mathscr{Y}$ with $C \notin \mathscr{Y}^{\prime}$, then $\left(\mathscr{Y}^{\prime}, C\right)$ is also a capture move. We frequently use this observation without explicitly mentioning it.

Lemma 6.2. Let $A, B$ and $C$ be distinct exceptional curves on $X$, with $A$ and $B$ disjoint, and let $D$ be any divisor. If $\mathrm{H}^{1}\left(X, O_{X}(D-A-B-C)\right)=0$, then $(\{A, B\}, C)$ is a capture move for $D$. In particular, if $\left(-K_{X}+D-A-B-C\right)$ is $n e f$, and if either it is big or it has anticanonical degree two, then $(\{A, B\}, C)$ is a capture move for $D$.

Proof. Since $A$ and $B$ are disjoint, there is an exact sequence of sheaves

$$
0 \rightarrow \mathrm{O}_{X}(-A-B) \rightarrow \mathrm{O}_{X}(-A) \oplus \mathrm{O}_{X}(-B) \rightarrow O_{X} \rightarrow 0 .
$$

Tensoring with $\mathrm{O}_{X}(D-C)$ we obtain the short exact sequence

$$
0 \rightarrow \mathrm{O}_{X}(D-A-B-C) \rightarrow \mathrm{O}_{X}(D-A-C) \oplus \mathrm{O}_{X}(D-B-C) \rightarrow O_{X}(D-C) \rightarrow 0
$$

and the desired surjectivity follows from the associated long exact sequence in cohomology and the assumption that

$$
\mathrm{H}^{1}\left(X, \mathrm{O}_{X}(D-A-B-C)\right)=0 .
$$

The last statement follows from the Kawamata-Viehweg vanishing theorem when $-K_{X}+D-A-B-C$ is nef and big. Otherwise $-K_{X}+D-A-B-C=Q$ for 
some conic $Q$ and the statement follows from the exact sequence

$$
0 \rightarrow \mathrm{O}_{X}\left(K_{X}\right) \rightarrow \mathrm{O}_{X}\left(K_{X}+Q\right) \rightarrow \mathrm{O}_{Q}\left(K_{X}+Q\right) \rightarrow 0,
$$

by considering the associated long exact sequence in cohomology.

Lemma 6.3. If $X$ is sweeping, then the pairs $\left(\mathfrak{b}, K_{1}\right)$ and $\left(\mathfrak{C}, K_{2}\right)$ are capture moves for all $D \neq-2 K_{X}$.

Proof. Since $D+K_{X} \neq-K_{X}$, it follows from Corollary 4.7 and Remark 4.8 that $\mathrm{H}^{0}\left(X, \mathrm{O}_{X}\left(D+K_{X}\right)\right)$ is spanned by global sections supported on exceptional curves. Thus the following map is surjective

$$
\bigoplus_{S \in \mathscr{C}} \mathrm{H}^{0}\left(X, \mathrm{O}_{X}\left(D-S+K_{X}\right)\right) \otimes \mathrm{H}^{0}\left(X, \mathrm{O}_{X}(S)\right) \longrightarrow \mathrm{H}^{0}\left(X, \mathrm{O}_{X}\left(D+K_{X}\right)\right)
$$

and $\left(\mathscr{C}, K_{1}\right)$ and $\left(\mathscr{C}, K_{2}\right)$ are capture moves for $D$.

Definition 6.4. A stopping criterion for a divisor $D$ is a set $\mathscr{Y} \subset \mathscr{G}$ such that the following complex is exact:

$$
\bigoplus_{\substack{C_{i}, C_{j} \in \mathcal{S} \\ i<j}} \mathrm{H}^{0}\left(X, \mathscr{O}_{X}\left(D-C_{i}-C_{j}\right)\right) \stackrel{d_{2}}{\rightarrow} \bigoplus_{C_{i} \in \mathscr{S}} \mathrm{H}^{0}\left(X, \mathscr{O}_{X}\left(D-C_{i}\right)\right) \stackrel{d_{1}}{\rightarrow} \mathrm{H}^{0}\left(X, \mathscr{O}_{X}(D)\right)
$$

Remark 6.5. By Lemma 5.1, a subset of $\mathscr{G}$ of cardinality two is a stopping criterion for any divisor $D$.

The name stopping criterion is motivated by Theorem 6.7: whenever we can capture all curves in a given degree $D$ by curves contained in a stopping criterion, then there are no relations in degree $D$. In this case, we may stop looking for capture moves.

Definition 6.6. Let $D \in \operatorname{Pic}(X)$ be a divisor class and let $M:=\left(E_{1}, \ldots, E_{n}\right)$ be a sequence of elements of $\mathscr{G}$; define $\mathscr{Y}_{i}:=\mathscr{G} \backslash\left\{E_{1}, E_{2}, \ldots, E_{i}\right\}$ for all $i \in\{1, \ldots, n\}$. We say that $D$ is capturable (by $\mathcal{M}$ ) if

(1) $\left(\mathscr{Y}_{i}, E_{i}\right)$ is a capture move for $D$ for all $i \in\{1, \ldots, n\}$,

(2) $\mathscr{Y}_{n}$ is a stopping criterion for $D$.

Theorem 6.7. Fix a divisor class $D \in \operatorname{Pic}(X)$. If $D$ is capturable then

$$
b_{1, D}(\operatorname{Cox}(X))=0
$$

and hence there are no minimal generators of the ideal $I_{X}$ in degree $D$.

Proof. Let $\mathcal{M}:=\left(E_{1}, \ldots, E_{n}\right)$ be such that $D$ is capturable by $\mathcal{M}$. Let $\mathscr{Y}_{0}=\mathscr{G}$ and, for $i \in\{1, \ldots, n\}$ define $\mathscr{Y}_{i}:=\mathscr{G} \backslash\left\{E_{1}, E_{2}, \ldots, E_{i}\right\}$. We want to show that every cycle $\sigma$ is a boundary. Let $j(\sigma):=\max \left\{i \in\{0, \ldots, n\} \mid\|\sigma\| \subset \mathscr{S}_{i}\right\}$. By definition if $\|\sigma\| \subset \mathscr{Y}_{n}$, or equivalently if $j(\sigma)=n$, then $\sigma$ is a boundary. 
Suppose that $j=j(\sigma)<n$; since $\left(\mathscr{S}_{j+1}, E_{j+1}\right)$ is a capture move for $D$, we deduce as in (6-1) that $\sigma$ is homologous to a cycle $\tau$ such that $j(\tau) \geq j+1$. Repeating this argument we conclude that $\sigma$ is homologous to a cycle with support contained in $\mathscr{S}_{n}$ and thus that $\sigma$ is itself a boundary.

\section{Ample divisors of anticanonical degree at least four}

Let $X$ be a del Pezzo surface of degree one. Assume throughout this section that if $X$ is defined over a field of characteristic two, then $X$ is sweeping. In this section we prove that there are no minimal generators of the ideal $I_{X}$ in all sufficiently large ample degrees. More precisely, if $D$ is an ample divisor such that $-K_{X} \cdot D \geq 4$ then we show that $D$ is capturable.

The general strategy is the following. First we capture $K_{1}$ and $K_{2}$ using $\mathscr{b}$ via Lemma 6.3. Next, assume $C$ is an exceptional curve and we want to capture it using $\mathscr{S} \subset \mathscr{C}$. We prove that there exist disjoint exceptional curves $S$ and $T$ in $\mathscr{S}$ such that $\mathrm{H}^{1}\left(X, \mathrm{O}_{X}\left(K_{X}+L_{S T}\right)\right)=0$, where $L_{S T}:=-K_{X}+D-C-S-T$. Then $(\{S, T\}, C)$ is a capture move for $D$ by Lemma 6.2. Often we show that $L_{S T}$ is nef and either big or of anticanonical degree two, omitting any reference to Lemma 6.2. In all cases we capture enough curves to conclude that $D$ is a capturable divisor, using Lemma 5.1 as stopping criterion.

Lemma 7.1. Let $X$ be a del Pezzo surface of degree one and let $b: X \rightarrow Y$ be a birational morphism. Denote by $K_{Y}$ the pull-back to $X$ of a canonical divisor on $Y$ and let $N$ be a nef divisor on $X$. Assume that $\operatorname{deg}(Y) \geq 3$; then the divisor $D=-K_{X}-K_{Y}+N$ is capturable.

Proof. Let $\mathscr{S}$ be the set of exceptional curves contracted by $b$ and note that $\mathscr{S}$ consists of at least two disjoint exceptional curves and that $-K_{Y}-S-T$ is big and nef for all $S, T \in \mathscr{S}, S \neq T$. Let $C \in \mathscr{C} \backslash \mathscr{S}$ be any exceptional curve and let $C^{\prime}:=-2 K_{X}-C$. First we capture the curves $C$ such that $C^{\prime} \in \mathscr{S}$ using $\mathscr{Y}$ : $L_{C^{\prime} T}:=-K_{Y}+N-T$ is big and nef for any choice of $T \in \mathscr{S} \backslash\left\{C^{\prime}\right\}$.

Second we choose any two distinct $S, T \in \mathscr{Y}$, we let $\mathscr{S}^{\prime}:=\{S, T\}$ and we capture all curves $C \in \mathscr{C} \backslash \mathscr{S}^{\prime}$ such that $C^{\prime} \notin \mathscr{S}$ : the divisor $L_{S T}=C^{\prime}+\left(-K_{Y}-S-T\right)+N$ is big and nef provided $C^{\prime} \cdot\left(-K_{Y}-S-T\right)>0$; since the only curves orthogonal to $\left(-K_{Y}-S-T\right)$ are the curves in $\mathscr{S} \backslash \mathscr{S}^{\prime}$, we conclude.

Lemma 7.2. Let $X$ be a del Pezzo surface of degree one and let $b: X \rightarrow Y$ be $a$ birational morphism. Denote by $A$ the pull-back to $X$ of the minimal ample divisor on $Y$ and let $N$ be a nef divisor on $X$. Assume that $\operatorname{deg}(Y) \geq 8$; then the divisor $D=-K_{X}+A+N$ is capturable.

Proof. Note that $Y$ is isomorphic to $\mathbb{P}^{2}, B l_{p}\left(\mathbb{P}^{2}\right)$ or $\mathbb{P}^{1} \times \mathbb{P}^{1}$. 
Case 1: $Y \not 千 \mathbb{P}^{1} \times \mathbb{P}^{1}$. In this case $A=L+Q$, where $L$ is a twisted cubic and $Q$ is either zero or a conic. Thus it suffices to treat the case $D=-K_{X}+L+N$.

Let $\mathscr{S}$ be the set of curves contracted by $L$; thus $\mathscr{Y}$ consists of eight disjoint exceptional curves. Note that $\sum_{S \neq T \in \mathscr{Y}} L-S-T=7\left(-K_{X}+L\right)$ has intersection number at least 7 with every exceptional curve on $X$. Since the intersection number of two exceptional curves on $X$ is at most 3, it follows that every exceptional curve on $X$ intersects positively at least two of the curves $\{L-S-T \mid S \neq T \in \mathscr{Y}\}$. For any $C \in \mathscr{C} \backslash \mathscr{S}$ let $S, T \in \mathscr{S}$ be such that $\left(-2 K_{X}-C\right) \cdot(L-S-T)>0$ and such that no (rational) multiple of $N$ equals $\left(-2 K_{X}-C\right)+(L-S-T)$. Note that the second condition ensures that $N$ does not contract both $-2 K_{X}-C$ and $(L-S-T)$ when $-2 K_{X}-C+(L-S-T)$ is a conic. We have that $L_{S T}:=$ $-2 K_{X}+L-C-S-T+N=\left(-2 K_{X}-C\right)+(L-S-T)+N$ is nef and either it has anticanonical degree two or it is also big. Thus $(\mathscr{Y}, C)$ is a capture move for all $C \in \mathscr{C} \backslash \mathscr{Y}$. Let $S, T \in \mathscr{S}$ be distinct elements and let $\mathscr{S}^{\prime}:=\{S, T\}$. For any $C \in \mathscr{S} \backslash \mathscr{S}^{\prime}$ the divisor $L_{S T}:=\left(-2 K_{X}-C\right)+(L-S-T)+N$ is big and nef since $\left(-2 K_{X}-C\right) \cdot(L-S-T)=2$.

Case 2: $Y \simeq \mathbb{P}^{1} \times \mathbb{P}^{1}$. Let $\mathscr{Y}$ be the set of curves contracted by $A$; thus $\mathscr{Y}$ consists of seven disjoint exceptional curves. Note that $\sum_{S \neq T \in \mathscr{S}} A-S-T=-6 K_{X}+9 A$ has intersection number at least six with every exceptional curve on $X$ and that the summands are conics. Since the intersection number of an exceptional curve and a conic on $X$ is at most four, it follows that every exceptional curve on $X$ intersects positively at least two of the conics $\{A-S-T \mid S \neq T \in \mathscr{S}\}$. For any $C \in \mathscr{C} \backslash \mathscr{Y}$ let $S, T \in \mathscr{Y}$ be such that $\left(-2 K_{X}-C\right) \cdot(A-S-T)>0$. We have that $L_{S T}:=-2 K_{X}+A-C-S-T+N=\left(-2 K_{X}-C\right)+(A-S-T)+N$ is big and nef. Thus $(\mathscr{Y}, C)$ is a capture move for all $C \in \mathscr{C} \backslash \mathscr{Y}$. Let $S, T \in \mathscr{Y}$ be distinct elements and let $\mathscr{S}^{\prime}:=\{S, T\}$. For any $C \in \mathscr{Y} \backslash \mathscr{S}^{\prime}$ the divisor

$$
L_{S T}:=\left(-2 K_{X}-C\right)+(A-S-T)+N
$$

is big and nef since $\left(-2 K_{X}-C\right) \cdot(A-S-T)=4$.

Lemma 7.3. Let $X$ be a del Pezzo surface of degree one, $E$ an exceptional curve on $X$ and $N \neq 0$ a nef divisor on $X$ such that $N \cdot E=0$. The divisor $-(n+2) K_{X}+E+N$ is capturable for $n \geq 0$.

Proof. By Lemma 3.4, for $i \in\{1,2\}$, there is an exceptional curve $F_{i}$ on $X$ such that $F_{i} \cdot E=0$ and $N-F_{i}$ is either nef or the sum of a nef divisor and an exceptional curve, with $F_{1} \neq F_{2}$, . Let $\mathscr{Y}:=\left\{E, F_{1}, F_{2}\right\}$. For any $C \in \mathscr{C} \backslash \mathscr{Y}$, let $F \in\left\{F_{1}, F_{2}\right\}$ be such that $\left(-2 K_{X}-C\right)$ is not a fixed component of $(N-F)$; we have that $L_{E F}=-(n+3) K_{X}+E-C-E-F+N=-K_{X}+\left(-2 K_{X}-C\right)+(N-F)-n K_{X}$ is big and nef and we conclude that we can capture all curves in $\mathscr{C} \backslash \mathscr{Y}$, using only 
the three curves in $\mathscr{Y}$. Finally, by the same argument, we find $\left(\left\{E, F_{1}\right\}, F_{2}\right)$ is a capture move and we are done.

Lemma 7.4. Let $X$ be a del Pezzo surface of degree one, $E$ an exceptional curve on $X$ and $N$ a nef divisor on $X$. The divisor $D:=-(n+3) K_{X}+E+N$ is capturable for $n \geq 0$.

Proof. Let $E, E_{2}, \ldots, E_{8}$ be eight disjoint exceptional curves on $X$ and let $\tilde{E}_{i}:=$ $-K_{X}+E-E_{i}$ for $2 \leq i \leq 8$. Let $\mathscr{S}:=\left\{E, E_{2}, \ldots, E_{8}, \tilde{E}_{2}, \ldots, \tilde{E}_{8}\right\}$. It is clear from Table 1 that every exceptional curve $C \in \mathscr{C} \backslash \mathscr{Y}$ intersects positively at least two of the curves in $\mathscr{Y}$; in particular for every $C \in \mathscr{C} \backslash \mathscr{Y}$ there is $S \in \mathscr{Y}$ such that $S \cdot E=0, S \cdot C>0$ and $N$ is not a (rational) multiple of $-4 K_{X}-(S+C)$. Thus $L_{E S}=-K_{X}+D-E-S-C=\left(-4 K_{X}-C-S\right)+N-n K_{X}$ is nef and it either has anticanonical degree two or it is big. In either case $\mathrm{H}^{1}\left(X, \mathcal{O}_{X}\left(K_{X}+L_{E S}\right)\right)=0$ and we may capture all the curves in $\mathscr{C} \backslash \mathscr{Y}$ using the curves in $\mathscr{Y}$.

Note that for all integers $i, j, k, l \in\{2,3, \ldots, 8\}, i \neq j, k \neq l$, we have

$$
\left(-4 K_{X}-E_{i}-\tilde{E}_{j}\right) \cdot\left(-4 K_{X}-E_{k}-\tilde{E}_{l}\right)=0
$$

if and only if $i=k$ and $j=l$. In particular, if $N$ is a multiple of a conic, it is proportional to at most one of the divisors $\left(-4 K_{X}-E_{i}-\tilde{E}_{j}\right)$; relabeling the indices if necessary, we may assume that $N$ is not proportional to $-4 K_{X}-\left(E_{i}+\tilde{E}_{j}\right)$ for all $i, j \in\{2,3, \ldots, 8\}$ and $i \neq j,(i, j) \neq(7,8)$. Thus for all $i \in\{3,4, \ldots, 8\}$ the divisor $L_{E \tilde{E}_{2}}=\left(-4 K_{X}-E_{i}-\tilde{E}_{2}\right)+N-n K_{X}$ is either a conic or big and nef and we may capture $E_{3}, E_{4}, \ldots, E_{8}$ using $E, \tilde{E}_{2}$. Similarly we can capture $\tilde{E}_{2}, \tilde{E}_{3}, \ldots, \tilde{E}_{8}$ using $E, E_{2}$ and we are done.

Lemma 7.5. Let $X$ be a del Pezzo surface of degree one. Let $D=-(n+4) K_{X}+N$ where $n \geq 0$ and $N$ is a nef divisor. Let $C, S$ and $T$ be exceptional curves such that $S \cdot T=0$ and that $C \cdot S, C \cdot T$ are at least 2 . Then $(\{S, T\}, C)$ is a capture move for $D$ if either

(1) $C \cdot(S+T)=5$, or

(2) $C \cdot(S+T)=4$ and the divisor $N$ is either 0 or not a multiple of the conic $T^{\prime}+S^{\prime}+C^{\prime}+K_{X}$

Proof. Consider $L_{S T}:=-K_{X}+D-S-T-C=S^{\prime}+T^{\prime}+C^{\prime}-(n-1) K_{X}+N$. Since the inequalities $C^{\prime} \cdot S^{\prime} \geq 2$ and $C^{\prime} \cdot T^{\prime} \geq 2$ hold, the curves $S^{\prime}, C^{\prime}$ and $T^{\prime}$, taken together, are not all pullbacks of exceptional curves on a del Pezzo surface $Y$ of degree at least two. It follows that every exceptional curve $V \notin\left\{S^{\prime}, T^{\prime}, C^{\prime}\right\}$ intersects at least one curve in this set and thus $L_{S T}$ is nef. If $C \cdot(S+T)=5$ then either $C^{\prime}+S^{\prime}=-2 K_{X}$ or $C^{\prime}+T^{\prime}=-2 K_{X}$ and $L_{S T}$ is big. If $N=0$ then $L_{S T}$ has anticanonical degree 2 and capturability follows from Lemma 6.2. Finally, 
assume that $C \cdot S=C \cdot T=2$. In this case $S^{\prime}+T^{\prime}+C^{\prime}+K_{X}=Q$ is a conic and $L_{S T}=Q-n K_{X}+N$ is big unless $N=m Q$ for some $m \geq 1$.

Lemma 7.6. Let $X$ be a del Pezzo surface of degree one and let $N$ be a nef divisor on $X$. The divisor $-(n+4) K_{X}+N$ is capturable for $n \geq 0$.

Proof. Let $L$ be a twisted cubic and let $E_{1}, \ldots, E_{8}$ be exceptional curves on $X$ contracted by $L$. First we capture all curves $C \in \mathscr{C}$ such that $L \cdot C=3$. From Table 1 it is clear that $C=-K_{X}+E_{i}-E_{j}$ for some $i \neq j$. Set

$S=E_{j}, \quad T_{1}=2 L-E_{i}-E_{i_{1}}-E_{i_{2}}-E_{i_{3}}-E_{i_{4}}, \quad T_{2}=2 L-E_{i}-E_{j_{1}}-E_{j_{2}}-E_{j_{3}}-E_{j_{4}}$,

with $\{i, j\} \cap\left\{i_{1}, \ldots, i_{4}\right\}=\varnothing,\{i, j\} \cap\left\{j_{1}, \ldots, j_{4}\right\}=\varnothing,\left\{i_{1}, \ldots, i_{4}\right\} \neq\left\{j_{1}, \ldots, j_{4}\right\}$. Note that $L \cdot S, L \cdot T_{i} \neq 3, C \cdot S=C \cdot T_{i}=2$ and $S \cdot T_{i}=0$ for $i=1,2$. Let $T$ be an exceptional curve in $\left\{T_{1}, T_{2}\right\}$ such that $N \neq m\left(T^{\prime}+S^{\prime}+C^{\prime}+K_{X}\right)$. By Lemma 7.5(2), $(\{S, T\}, C)$ is a capture move.

Next, we capture curves $C$ with $L \cdot C \in\{4,5\}$. It is clear from Table 1 that for any such curve there exists a pair of distinct exceptional curves $S, T \in\left\{E_{1}, \ldots, E_{8}\right\}$ such that $(\{S, T\}, C)$ is a capture move via Lemma 7.5(2). Similarly we capture curves $C$ with $L \cdot C \in\{1,2\}$ using some pair $S, T \in\left\{E_{1}^{\prime}, \ldots, E_{8}^{\prime}\right\}$. Next we capture $E_{3}, \ldots, E_{8}$ using $E_{1}^{\prime}$ and $E_{2}^{\prime}$ via Lemma 7.5(1) and finally, we capture $E_{1}^{\prime}, \ldots, E_{8}^{\prime}$ using $E_{1}$ and $E_{2}$. This concludes the proof.

Lemma 7.7. Let $X$ be a del Pezzo surface of degree one and let $Q$ be a conic on $X$. The divisor $D=-(n+1) K_{X}+(m+1) Q$ is capturable for $m, n \geq 0$ and $m+n \geq 1$.

Proof. Suppose first that $n \geq 1$. Let $\mathscr{Y}$ be the set of curves contracted by $Q$ and for any $S \in \mathscr{Y}$, let $\tilde{S}$ be the unique curve such that $S+\tilde{S}=Q$. If $S, T, C$ are exceptional curves let $B_{S T}=-K_{X}+C^{\prime}+Q-S-T$ and note that if $B_{S T}$ is nef and big then so is $L_{S T}:=-K_{X}+D-C-S-T=B_{S T}+m Q-(n-1) K_{X}$ because $n \geq 1$. Moreover $B_{S T}$ has anticanonical degree 2 and $h^{2}\left(X, \mathcal{O}_{X}\left(B_{S T}\right)\right)=0$ so by Riemann-Roch $B_{S T}$ is nef if and only if $B_{S T}^{2}=2 Q \cdot C^{\prime}-2 C^{\prime} \cdot S-2 C^{\prime} \cdot T \geq 0$.

We first show that the curves $C$ in $\mathscr{C} \backslash \mathscr{S}$ can be captured with the curves in $\mathscr{Y}$. To do so, we split them into cases according to the value of $Q \cdot C^{\prime}$.

If $Q \cdot C^{\prime}=0$ choose $T \in \mathscr{Y}$ disjoint from $C^{\prime}$. In this case $B_{C^{\prime} T}=-K_{X}+\tilde{T}$ is nef and big, and $\left(\left\{C^{\prime}, T\right\}, C\right)$ is a capture move. If $Q \cdot C^{\prime}=1$ choose disjoint curves $S$ and $T \in \mathscr{Y}$ which are also disjoint from $C^{\prime}$ and note that $B_{S T}$ has anticanonical degree 2 and square 2 so it is the pullback to $X$ of the anticanonical divisor of a del Pezzo surface of degree 2 and thus it is nef and big; whence $(\{S, T\}, C)$ is a capture move. If $Q \cdot C^{\prime}=2$, by Lemma 3.5 there exist disjoint curves $S, T$ in $\mathscr{Y}$ such that $S \cdot C^{\prime}=T \cdot C^{\prime}=0$ so $B_{S T}$ has square 4 and hence $B_{S T}=-2 K$ is nef and big. Thus $(\{S, T\}, C)$ is a capture move in this case. Finally if $Q \cdot C^{\prime}=3$, then let $S$ and $T$ be disjoint curves in $\mathscr{Y}$ such that $S \cdot C^{\prime}=T \cdot C^{\prime}=1$; then $B_{S T}$ has square 2 and it is a nef and big divisor since it is the pullback to $X$ of the anticanonical 
divisor of a del Pezzo surface of degree 2. Thus $(\{S, T\}, C)$ is a capture move in this case.

Now choose $S, T$ disjoint exceptional curves in $\mathscr{Y}$. We show that all curves $C$ in $\mathscr{S} \backslash\{S, T\}$ can be captured from $\{S, T\}$. In this case $Q \cdot C^{\prime}=4$ and $S \cdot C^{\prime}$, $T \cdot C^{\prime} \leq 2$, so $B_{S T}^{2} \geq 0$. If $B_{S T}$ is big the statement follows since so is $L_{S T}$. If $B_{S T}^{2}=0$ there are two cases to consider, either $n=1$ and $m=0$ and the statement follows from Lemma 6.2, or at least one of $n, m$ is greater than one. In this case $L_{S T}=B_{S T}-(n-1) K_{X}+m Q$ is big because $B_{S T} \cdot Q=6 \neq 0$. It follows that we can capture every exceptional curve in $\mathscr{S} \backslash\{S, T\}$ from $\{S, T\}$.

Suppose now that $n=0$ and $m \geq 1$. Note that if $Q \cdot C^{\prime} \leq 1$ then

$$
Q \cdot(D-C)=Q \cdot\left(K_{X}+C^{\prime}+(m+1) Q\right)=-2+Q \cdot C^{\prime}<0,
$$

so $D-C$ is not an effective divisor. In this case $C$ is captured vacuously and thus we restrict our attention to curves $C$ with $Q \cdot C^{\prime} \geq 2$. Let $\mathscr{S}$ be the set of exceptional curves contracted by $Q$ and for any $S \in \mathscr{S}$ let $\tilde{S}$ be the unique curve such that $S+\tilde{S}=Q$.

For any $C \in \mathscr{C} \backslash \mathscr{S}$ which satisfies $Q \cdot C^{\prime} \geq 2$ there exist disjoint curves $S, T$ in $\mathscr{Y}$ such that $\tilde{S}$ and $\tilde{T}$ intersect $C^{\prime}$. By construction $\tilde{S}+\tilde{T}+C^{\prime}$ is nef and big and so is $L_{S T}:=-K_{X}+D-S-T-C=\tilde{S}+\tilde{T}+C^{\prime}+(m-1) Q$. It follows that we can capture $\mathscr{C} \backslash \mathscr{Y}$ with $\mathscr{S}$.

Finally, fix two disjoint curves $S, T \in \mathscr{Y}$ and let $C$ be any curve in $\mathscr{S} \backslash\{S, T\}$. Since $Q \cdot C=0$ we have $Q \cdot C^{\prime}=4$ so $C^{\prime}$ intersects both $\tilde{S}$ and $\tilde{T}$. It follows that $L_{S T}$ is nef and big and that $\mathscr{Y}$ can be captured from $\{S, T\}$.

Theorem 7.8. Let $X$ be a sweeping del Pezzo surface of degree one and let $D$ be an ample divisor. If $-K_{X} \cdot D \geq 4$, then $D$ is capturable.

Proof. Write $D=-n K_{X}+N$, where $n \geq 1$, the divisor $N$ is nef and not ample and $n-K_{X} \cdot N \geq 4$. If $N=0$, then $n \geq 4$ and we conclude using Lemma 7.6. If $N \neq 0$, write $N=m A+N^{\prime}$ where $X \rightarrow Y$ is a morphism with connected fibers, $A$ is the pull-back to $X$ of the minimal ample divisor on $Y, N^{\prime}$ is the pull-back of a nef divisor on $Y$ and $m \geq 1$. If $Y$ is a surface, then we conclude using Lemmas 7.1-7.4. If $Y \simeq \mathbb{P}^{1}$, then we conclude using Lemma 7.7.

\section{Ample divisors of anticanonical degree three}

The only ample divisors $D$ that seem to elude the strategy of Section 7 are those of anticanonical degree three. These divisors are $-3 K_{X},-2 K_{X}+E,-K_{X}+Q$, where $E$ is any exceptional curve and $Q$ is any conic. Recall that there is a surjection

$$
\left(k[G] / J_{X}\right)_{D} \rightarrow\left(k[\varphi] / I_{X}\right)_{D}
$$


see Section 2. We show the dimension of $\left(k[G] / J_{X}\right)_{D}$ is at most $h^{0}\left(X, O_{X}(D)\right)=$ $\operatorname{dim}\left(k[G] / I_{X}\right)$ and conclude that $\left(I_{X}\right)_{D}=\left(J_{X}\right)_{D}$.

Lemma 8.1. Let $X$ be a del Pezzo surface of degree one and let $A, B, G$ be exceptional curves with $A+B=-K_{X}+G$. Then $k_{1} g, k_{2} g$ and ab form a basis for $\mathrm{H}^{0}\left(X, \mathrm{O}_{X}\left(-K_{X}+G\right)\right)$. In particular, if $C, D$ are exceptional curves with $C+D=-K_{X}+G$ then $c d=\alpha_{1} k_{1} g+\alpha_{2} k_{2} g+\alpha_{3} a b$ and the coefficient $\alpha_{3}$ is nonzero.

Proof. The morphism $X \rightarrow \mathbb{P}^{2}$ associated to $\left|-K_{X}+G\right|$ contracts $G$ and is ramified along a smooth plane quartic $R$. The image of $G$ is a point not lying on any bitangent to $R$ since otherwise $X$ would have had a (-2)-curve. The images of $K_{1}$ and $K_{2}$ in $\mathbb{P}^{2}$ are distinct lines through the image of $G$ and therefore the three lines in $\mathbb{P}^{2}$ corresponding to $A+B, K_{1}+G K_{2}+G$ have no common point and are independent.

Lemma 8.2. Let $X$ be a del Pezzo surface of degree one; then the ideal $I_{X}$ has no minimal generators in degree $D=-3 K_{X}$.

Proof. The only ways of writing $-3 K_{X}$ as a sum of three effective divisors are given in the following table.

\begin{tabular}{|l|l|}
\hline Monomial & \multicolumn{1}{|c|}{ Description } \\
\hline$h_{1} h_{2} h_{3}$ & $h_{1}, h_{2}, h_{3} \in\left\{k_{1}, k_{2}\right\}$ \\
\hline$h a a^{\prime}$ & $h \in\left\{k_{1}, k_{2}\right\}, A \in \mathscr{C}$ \\
\hline$a b c$ & $\begin{array}{l}A, B, C \in \mathscr{C} \\
A \cdot B=A \cdot C=B \cdot C=2\end{array}$ \\
\hline
\end{tabular}

Indeed, let $-3 K_{X}=A+B+C$ be any expression of $-3 K_{X}$ as a sum of three effective divisors. If one among $A, B, C$ is in $\left|-K_{X}\right|$, then we are in one of the first two cases above. If $A, B, C \in \mathscr{C}$, then intersecting with $A, B, C$ successively both sides of the equation $-3 K_{X}=A+B+C$ we obtain the system

$$
\left\{\begin{array}{l}
A \cdot B+A \cdot C=4, \\
A \cdot B+B \cdot C=4, \\
A \cdot C+B \cdot C=4,
\end{array}\right.
$$

whose only solution is $A \cdot B=A \cdot C=B \cdot C=2$.

By definition $\left(I_{X}\right)_{2}=\left(J_{X}\right)_{2}$; we deduce that the span of the monomials of the first two forms in (8-1) in $k[\mathscr{G}] / J_{X}$ has dimension at most six, being the image of $\mathrm{H}^{0}\left(X, \mathrm{O}_{X}\left(-K_{X}\right)\right) \otimes \mathrm{H}^{0}\left(X, \mathrm{O}_{X}\left(-2 K_{X}\right)\right)$ under the multiplication map (note that $\operatorname{dim} \mathrm{H}^{0}\left(X, \mathrm{O}_{X}\left(-K_{X}\right)\right)=2, \operatorname{dim} \mathrm{H}^{0}\left(X, \mathrm{O}_{X}\left(-2 K_{X}\right)\right)=4$ and the linearly independent elements $k_{1} \otimes k_{2}^{2} e-k_{2} \otimes k_{1} k_{2}$ and $k_{2} \otimes k_{1}^{2} e-k_{1} \otimes k_{1} k_{2}$ are in the kernel). 
Fix any monomial $p=a b c$ of the third form in (8-1) and denote the span of $p$ together with the monomials of the first two forms in (8-1) by $V$. Let $q=\operatorname{def}$ be any monomial of the third form in (8-1); we prove that $q$ can be written as the sum of an element of $V$ and an element of $J_{X}$.

The result is clear if $c=f$, since in this case we can use the relation coming from degree $A+B$ involving the monomials $k_{1} g c, k_{2} g c, a b c$ and $d e c$ to conclude (see Lemma 8.1). By the same reasoning we reduce to the case in which $\{a, b, c\}$ and $\{d, e, f\}$ are disjoint.

Suppose that $A \cdot D=2$; then the divisor $G:=-3 K_{X}-A-D$ satisfies $G^{2}=$ $K_{X} \cdot G=-1$, and hence $G$ is an exceptional curve. Moreover from the fact that $D \cdot G=2$, we deduce that $G$ is an exceptional curve on the del Pezzo surface obtained by contracting $D^{\prime}$ and whose anticanonical divisor pulled-back to $X$ is $E+F$; let $H:=E+F-G$ and note that $H$ is also an exceptional curve. In particular we can use a relation coming from degree $E+F$ involving the monomials $d k_{1} d^{\prime}$, $d k_{2} d^{\prime}, d g h$ and $d e f$ to reduce to the case $\{a, b, c\} \cap\{d, e, f\} \neq \varnothing$.

Finally, if $A \cdot D=A \cdot E=A \cdot F=1$, then $A$ is a conic in the del Pezzo surface obtained by contracting $F^{\prime}$. From Table 1 it follows that there are exceptional curves intersecting any given conic twice; denote the strict transform in $X$ of one such curve of $X$ by $H$ and the exceptional curve $E+F-H$ by $J$. Thus we can use a relation coming from degree $E+F$ involving the monomials $d k_{1} d^{\prime}, d k_{2} d^{\prime}$, $d h j$ and $d e f$ to reduce to the case in which $A \cdot D=2$.

Lemma 8.3. Let $E$ be an exceptional curve on $X$; then the ideal $I_{X}$ has no minimal generators in degree $D=-2 K_{X}+E$.

Proof. The monomials of degree $D$ are of the following forms:

\begin{tabular}{|l|l|}
\hline Monomial & \multicolumn{1}{|c|}{ Description } \\
\hline$k s$ & $k \in\left\{k_{1}, k_{2}\right\}, s \in k\left[\varphi_{-K_{X}+E}\right.$ \\
\hline$a a^{\prime} e$ & $A \cdot A^{\prime}=3$ \\
\hline & $A \cdot E=1$ \\
& $B \cdot E=C \cdot E=0$ \\
$a b c$ & $A \cdot B=A \cdot C=2$ \\
& $B \cdot C=1$ \\
\hline
\end{tabular}

Indeed let $m \in k[G]_{D}$ be a monomial. If $k \in\left\{k_{1}, k_{2}\right\}$, or $e$ divides $m$, then $m$ is of one of the first two forms. Otherwise let $m=a b c$, with $\{a, b, c\} \cap\left\{k_{1}, k_{2}, e\right\}=\varnothing$; if two of the curves $A, B, C$ have intersection number three, then the remaining one is $E$, which we are excluding. If the intersection numbers among the curves $A, B, C$ are all at most two, then the required conditions follow multiplying successively the equality $A+B+C=-2 K_{X}+E$ by $A, B, C$. 
We show that the monomials of degree $D$ span a subspace of dimension at most six of $\left(k[G] / J_{X}\right)_{D}$; since $\operatorname{dim} \operatorname{Cox}(X)_{D}=6$, the result follows.

First, the image of $\mathrm{H}^{0}\left(X, \mathcal{O}_{X}\left(-K_{X}\right)\right) \otimes \mathrm{H}^{0}\left(X, \mathscr{O}_{X}\left(-K_{X}+E\right)\right)$ in $k[G]_{D}$ has dimension at most five: $\operatorname{dim} \mathrm{H}^{0}\left(X, \mathrm{O}_{X}\left(-K_{X}\right)\right)=2, \operatorname{dim}^{0}\left(X, \mathcal{O}_{X}\left(-K_{X}+E\right)\right)=3$ and the element $k_{1} \otimes k_{2} e-k_{2} \otimes k_{1} e$ is in the kernel.

Second, the span of the monomials of the first two forms has dimension at most six. Let $a a^{\prime} e, b b^{\prime} e$ be any two monomials of the second form. There is a quadratic relation $q$ involving $a a^{\prime}, b b^{\prime}, k_{1}^{2}, k_{1} k_{2}, k_{2}^{2}$ since these five vectors correspond to five elements of the four dimensional space $\mathrm{H}^{0}\left(X, \mathrm{O}_{X}\left(-2 K_{X}\right)\right)$. Moreover $a a^{\prime}$ and $b b^{\prime}$ are independent from $k_{1}^{2}, k_{1} k_{2}, k_{2}^{2}$, since the monomials in $k_{1}, k_{2}$ correspond to sections having a base-point, and neither of the remaining elements $a a^{\prime}$ and $b b^{\prime}$ vanishes at the base-point. Thus in the relation $q$ the coefficients of $a a^{\prime}$ and $b b^{\prime}$ are both nonzero. We deduce that the span of the elements of the first two forms has dimension at most six in $\left(k[G] / J_{X}\right)_{D}$.

Third, let $a b c$ be a monomial of the third form. The divisor $A+B$ contracts the unique exceptional curve $F:=K_{X}+A+B$, and the divisor $G:=A+B-E$ is an exceptional curve on the del Pezzo surface $Y$ obtained from $X$ by contracting $F$. Therefore there is a quadratic relation involving the monomials $a b, g e, k_{1} f, k_{2} f$ and the monomials $a b$ and $g e$ are independent from $k_{1} f, k_{2} f$, by Lemma 8.1. Thus we may use this relation to write the image of $a b c$ in $k[G] / J_{X}$ as a combination of divisors of the first two forms and the proof is complete.

Lemma 8.4. Let $Q$ be a conic on $X$; then the ideal $I_{X}$ has no minimal generators in degree $D=-K_{X}+Q$.

Proof. The monomials of degree $D$ are of the following forms:

\begin{tabular}{|l|l|}
\hline Monomial & \multicolumn{1}{|c|}{ Description } \\
\hline$k s$ & $k \in\left\{k_{1}, k_{2}\right\}, s \in k\left[G_{Q}\right]_{Q}$ \\
\hline & $E_{1} \cdot Q=0$ \\
& $E \cdot F=2$ \\
$e f e_{1}$ & $E_{1} \cdot(E+F)=2$ \\
& $Q \cdot E=Q \cdot F=1$ \\
\hline & $E_{1} \cdot Q=E_{2} \cdot Q=E_{1} \cdot E_{2}=0$ \\
& $Q \cdot E=E_{1} \cdot E=E_{2} \cdot E=2$ \\
\hline
\end{tabular}

Note that $E_{1}, E_{2}$ are disjoint components of reducible fibers of the conic bundle associated to $Q$. Indeed let

$$
-K_{X}+Q \sim A+B+C
$$


Multiplying both sides of (8-2) by $Q$ we find

$$
2=A \cdot Q+B \cdot Q+C \cdot Q
$$

thus at least one of the intersection products is zero. Suppose that $C \cdot Q=0$, and let $\tilde{C}=Q-C$; then $A+B=-K_{X}+\tilde{C}$ is a reducible divisor in the linear system associated to the pull-back of the anticanonical divisor on the del Pezzo surface of degree two obtained from $X$ by contracting $\tilde{E}$. The first case in (8-2) corresponds to the sections containing a divisor in the linear system $\left|-K_{X}\right|$; the second to one not containing it and containing two curves not in the linear system $|Q|$; the last one to one containing two elements of $|Q|$.

Since $\left(k[G] / J_{X}\right)_{2}=\operatorname{Cox}(X)_{2}$, it follows that the dimension of the span of the monomials of the first form in (8-2) modulo $\left(J_{X}\right)_{-K_{X}+Q}$ is at most four. Let $p=e e_{1} e_{2}$ be a monomial in $k[\varphi]_{-K_{X}+Q}$ of the third form in (8-2) and let $V$ denote the span of the monomials of the first form in (8-2) together with $p$ in $\left(k[G] / J_{X}\right)_{-K_{X}+Q}$. Since $\operatorname{dim}\left(\operatorname{Cox}(X)_{-K_{X}+Q}\right)=5$, the result follows if we show that

$$
V=\left(k[G] / J_{X}\right)_{-K_{X}+Q} .
$$

Let $q=\bar{e} \bar{e}_{1} \bar{e}_{2}$ be a monomial in $k\left[\varphi^{C}\right]_{-K_{X}+Q}$ of the third form in (8-2).

If $\bar{e}_{1}=e_{1}$, then let $E_{3}=K_{X}+E+E_{2}=K_{X}+\bar{E}+\bar{E}_{2}$ be the exceptional curve contracted by $E+E_{2}$. By Lemma 8.1, applied to the monomials $\bar{e} \bar{e}_{2}, e e_{2}, k_{1} e_{3}, k_{2} e_{3}$, we conclude that the image of $q$ in $\left(k[G] / J_{X}\right)_{-K_{X}+Q}$ belongs to $V$.

If $\left\{\bar{E}_{1}, \bar{E}_{2}\right\} \cap\left\{E_{1}, E_{2}\right\}=\varnothing$, then at least one curve in $\left\{\bar{E}_{1}, \bar{E}_{2}\right\}$ is disjoint from one curve in $\left\{E_{1}, E_{2}\right\}$ and relabeling the indices if necessary we may assume that $\bar{E}_{1} \cdot E_{1}=0$. By the same reasoning above $q=\bar{e} \bar{e}_{1} \bar{e}_{2}$ is in the span of $V$ and $\tilde{q}=\tilde{e} \bar{e}_{1} e_{1}$ (note that $\tilde{E}:=-K_{X}+Q-\bar{E}_{1}-E_{1}$ is an exceptional curve, since it has anticanonical degree one and square negative one), and we conclude since $\tilde{q}$ is in the span of $V$.

Finally, if $q=\bar{e} \bar{f} \bar{e}_{1}$, then at least one curve in $E_{1}, E_{2}$ is disjoint from $\bar{E}_{1}$ and relabeling the indices if necessary we may assume that $\bar{E}_{1} \cdot E_{1}=0$. Reasoning as above, $q$ is in the span of $V$ and $\tilde{e} e_{1} \bar{e}_{1}$, and we are done.

Corollary 8.5. Let $X$ be a sweeping del Pezzo surface of degree one and let $D$ be an ample divisor on $X$ such that $-K_{X} \cdot D \geq 3$. Then the ideal $I_{X}$ has no minimal generators in degree $D$.

Proof. By Theorem 7.8, if $-K_{X} \cdot D \geq 4$ then $D$ is capturable and the result follows from Theorem 6.7. Since the only ample divisors of anticanonical three are $-3 K_{X}$, $-2 K_{X}+E$ and $-K_{X}+Q$, where $E$ is an exceptional curve and $Q$ is a conic, the result is true if $-K_{X} \cdot D=3$, by Lemmas 8.2-8.4. 


\section{Nonample divisors}

In this section we prove that the relations in degree $D$, where $D$ is a nonample divisor on $X$ of anticanonical degree at least three, coming from $J_{X}$ are sufficient to show that the quotient $\left(k[G] / J_{X}\right)_{D}$ is in fact spanned by monomials coming from a del Pezzo surface of smaller degree. This is the basis for the inductive procedure of Section 11. Throughout this section we assume that del Pezzo surfaces $X$ of degree one defined over fields of characteristic two are sweeping.

Lemma 9.1. Let $X$ be a del Pezzo surface of degree at most five and let $D$ be a divisor on $X$. Suppose that $E$ is an exceptional curve on $X$ such that $D \cdot E=0$. Then $\left(k[G] / J_{X}\right)_{D}$ is spanned by products of variables corresponding to exceptional curves disjoint from $E$.

Proof. Let $m \in k[\varphi]_{D}$ be a monomial and write $m=e^{p} \cdot s$, where $p \geq 0$ and $s$ is a product of variables different from $E$. Note that if $p=0$, then $s$ is a product of variables corresponding to divisors disjoint from $E$, since by assumption $D \cdot E=0$. We shall show that if $p \geq 1$, then using the quadratic relations we may decrease $p$; the result then follows by induction on $p$.

Suppose that $p \geq 1$. Since $D \cdot E=0$, there is a variable $c \in \mathscr{G}$ such that $E \cdot C>0$ and $c$ divides $s$. The monomial $c e$ is a monomial of anticanonical degree two corresponding to a nef divisor. By definition of $J_{X}$, the vector space $\left(k[G] / J_{X}\right)_{C+E}$ coincides with $\operatorname{Cox}(X)_{C+E}$; thus it suffices to show that there is a basis of $\operatorname{Cox}(X)_{C+E}$ consisting of sections vanishing along exceptional curves different from $E$. To conclude, we analyze all the possibilities for the divisor $C+E$. Case 1. The divisor $C+E$ is a conic. The linear system $|C+E|$ contains $8-\operatorname{deg}(X)$, i.e., at least three, distinct reducible elements and any two of these span it.

Case 2. The divisor $C+E$ is $-b^{*} K_{Y}$, where $b: X \rightarrow Y$ is a birational morphism, $Y$ has degree two. The linear system $|C+E|$ contains 28 distinct reducible elements and any five of these span it (Lemma 4.1).

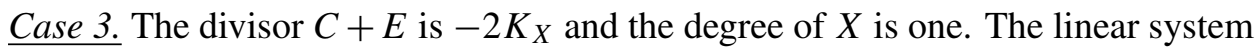
$\left|-2 K_{X}\right|$ contains 120 distinct reducible elements whose irreducible components are $(-1)$-curves and any 113 of these span it (Proposition 4.4).

In all these cases the sections supported on $(-1)$-curves distinct from $E$ span $\operatorname{Cox}(X)_{C+E}$, and the lemma follows.

Lemma 9.2. Let $X$ be a del Pezzo surface of degree at most five and let $D$ be a divisor on $X$. Suppose that $E$ is an exceptional curve on $X$ such that $D \cdot E<0$. Then $\operatorname{dim}\left(k[G] / J_{X}\right)_{D}=\operatorname{dim}\left(k[G] / J_{X}\right)_{D-E}$ and $\operatorname{dim} \operatorname{Cox}(X)_{D}=\operatorname{dim} \operatorname{Cox}(X)_{D-E}$.

Proof. If $D$ is not effective, then $D-E$ is not effective and $k[G]_{D}=k[\varphi]_{D-E}=0$. If $D$ is effective, then $E \cdot D<0$ implies that $E$ is a component of $D$ and thus every 
monomial in $k[G]_{D}$ is divisible by $e$; the same argument also proves the statement about the Cox ring.

\section{Del Pezzo surfaces of degree at least two}

Lemma 10.1. Let $X$ be a del Pezzo surface of degree two. Any ample divisor is capturable except $-K_{X}$.

Proof. Let $D \neq-K_{X}$ be an ample divisor and write $D=-n K_{X}+N$, with $n \geq 1$ and $N$ nef and not ample; by assumption either $n \geq 2$ or $N \neq 0$.

Suppose first that $N \neq 0$; if $N$ contracts more than one exceptional curve, then we let $\mathscr{S}$ be the set of exceptional curves contracted by $N$. If $N$ contracts exactly one exceptional curve $E$, then we let $F$ be any exceptional curve disjoint from $E$ and let $\mathscr{Y}:=\{E, F\}$.

Let $C \in \mathscr{C} \backslash \mathscr{S}$ and let $C^{\prime}:=-K_{X}-C$. If $C^{\prime} \in \mathscr{Y}$, then the divisor $L_{C^{\prime} T}=$ $N+\left(-K_{X}-T\right)-(n-1) K_{X}$ is big and nef for all $T \in \mathscr{Y}, T \neq C^{\prime}$ since

$$
\left(-K_{X}-T\right) \cdot N=-K_{X} \cdot N>0
$$

thus we can capture all such curves using $\mathscr{Y}$. If $C^{\prime} \notin \mathscr{Y}$, then let $S, T \in \mathscr{Y}$ be disjoint and note that $N-T$ is either nef or $N$ is a multiple of a conic and it is the sum of a multiple of a conic and a single exceptional curve. Moreover

- $S^{\prime} \cdot(N-T)=-K_{X} \cdot N-1>0$;

- if $N$ is a multiple of a conic, then $S^{\prime}$ also intersects the fixed component of $N-T$;

- $C^{\prime} \cdot(N-T)=-K_{X} \cdot N-1-C \cdot T$ is zero if and only if $N$ is a conic and $C \cdot T=1$.

In this last case, note that $C \cdot N \neq 0$, and $C$ intersects at least one component in every reducible fiber of the conic bundle determined by $N$; hence we may choose $T$ to be the other component if $C^{\prime} \cdot(N-T)=0$. In all cases we may choose $S, T \in \mathscr{Y}$ disjoint such that $L_{S T}$ is big and nef, and hence capture all $C \in \mathscr{C} \backslash \mathscr{Y}$ using only curves in $\mathscr{S}$. In particular, we are done if $N$ contracts at most two exceptional curves.

To conclude it suffices to treat the two cases $N=L+N^{\prime}$ and $N=Q+N^{\prime}$, where $L$ is a twisted cubic, $Q$ is a conic and $N^{\prime}$ is nef; note also that $S \cdot N^{\prime}=0$, for all $S \in \mathscr{Y}$.

Choose any two disjoint $S, T \in \mathscr{Y}$ and let $C \in \mathscr{Y}, C \neq S, T$. If $N=L+N^{\prime}$, we have that the divisor $L_{S T}=-K_{X}+\left(-K_{X}-C\right)+(L-S-T)+N^{\prime}$ is big and nef since $\left(-K_{X}-C\right) \cdot(L-S-T)=1$ and we conclude. If $N=Q+N^{\prime}$, then the divisor $L_{S T}=\left(-K_{X}-S\right)+\left(-K_{X}-T\right)+(Q-C)+N^{\prime}$ is big and nef since $\left(-K_{X}-S\right) \cdot(Q-C)=\left(-K_{X}-T\right) \cdot(Q-C)=1$ and again we conclude. 
Suppose now that $N=0$ and hence $D=-n K_{X}$, where $n \geq 2$. Let $\mathscr{Y}$ be any set of seven disjoint exceptional curves. For all $C \in \mathscr{C} \backslash \mathscr{Y}$, there are distinct $S, T \in \mathscr{Y}$ such that $C \cdot S, C \cdot T>0$ : this is clear from the list of exceptional curves. Thus for such a choice of $S, T$, we have that the divisor $L_{S T}=-3 K_{X}-C-S-T=C^{\prime}+S^{\prime}+T^{\prime}$ is big and nef and we may capture all the curves in $\mathscr{C} \backslash \mathscr{Y}$ using $\mathscr{Y}$. Finally let $S, T \in \mathscr{Y}$ be distinct elements and let $S^{\prime}:=-K_{X}-S, T^{\prime}:=-K_{X}-T$; for all $C \in \mathscr{S}$ the divisor $L_{S^{\prime} T^{\prime}}=-3 K_{X}-C-S^{\prime}-T^{\prime}=C^{\prime}+S+T$ is big and nef and we conclude.

Lemma 10.2. Let $X$ be a del Pezzo surface of degree three. Any ample divisor is capturable.

Proof. Let $D$ be an ample divisor and write $D=-K_{X}+N$, with $N$ a nef divisor.

Let $\mathscr{S}$ be a set of six disjoint exceptional curves. For all $C \in \mathscr{C} \backslash \mathscr{S}$ there are two distinct $S, T \in \mathscr{Y}$ such that $C \cdot S=C \cdot T=1$. With these choices of $S, T$ we have that the divisor $L_{S T}=-2 K_{X}+N-C-S-T=\left(-K_{X}-T\right)+\left(-K_{X}-C-S\right)+N$ is big and nef since $\left(-K_{X}-T\right) \cdot\left(-K_{X}-C-S\right)=1$. Thus we may capture all the exceptional curves $C \in \mathscr{C} \backslash \mathscr{Y}$ using the curves in $\mathscr{Y}$. Let $\mathscr{S}^{\prime}$ be the set of six disjoint exceptional curves each intersecting all the curves in $\mathscr{S}$ except for one (thus $\mathscr{Y} \cup \mathscr{Y}^{\prime}$ is a Schläfli double-six). Choose $S, T \in \mathscr{Y}$ and let $S^{\prime}, T^{\prime} \in \mathscr{Y}^{\prime}$ be two corresponding curves, that is $S \cdot S^{\prime}=T \cdot T^{\prime}=0$. Using the moves above we can capture $\left\{S^{\prime}, T^{\prime}\right\}$ with $\mathscr{S}$ and we can capture $\mathscr{S} \backslash\{S, T\}$ using $\mathscr{S}^{\prime} \backslash\left\{S^{\prime}, T^{\prime}\right\}$, and finally we capture $\mathscr{S}^{\prime} \backslash\left\{S^{\prime}, T^{\prime}\right\}$ using $\{S, T\}$.

Lemma 10.3. Let $X$ be a del Pezzo surface of degree four. Any ample divisor is capturable.

Proof. Let $D$ be an ample divisor and write $D=-K_{X}+N$, with $N$ a nef divisor. The criterion to capture curves is that $(\{E, F\}, C)$ is a capture move if $C \notin\{E, F\}$, $E \cdot F=0$ and $C \cdot E+C \cdot F>0$ : if $C \cdot E>0$, then the divisor

$$
L_{E F}=\left(-K_{X}-C-E\right)+\left(-K_{X}-F\right)+N
$$

is the sum of a conic, a twisted cubic and a nef divisor and hence it is big and nef.

We refer to Table 1 . Use $E_{3}, E_{4}$ to capture $L-E_{i}-E_{j}$ for all $3 \leq i<j<5$. Use $L-E_{1}-E_{3}, L-E_{1}-E_{4}, L-E_{1}-E_{5}$ to capture $E_{3}, E_{4}, E_{5}$ and finally use $E_{1}, E_{2}$ to capture all the remaining curves.

Lemma 10.4. Let $X$ be a del Pezzo surface of degree five. Any ample divisor is capturable.

Proof. Same criterion and strategy as in Lemma 10.3, ignoring any reference to the index 5.

Lemma 10.5. Let $X$ be a del Pezzo surface of degree six. Any ample divisor is capturable. 
Proof. Same criterion as in Lemma 10.3. Capture $L-E_{1}-E_{2}$ using $E_{1}, E_{2}$ and then capture the remaining curves using $L-E_{1}-E_{3}, L-E_{2}-E_{3}$.

\section{Quadratic generation}

Main result. We collect all the information gathered in the previous sections to prove the main result of this paper: the ideal $I_{X}$ is generated by its degree two part.

Proof of the Batyrev-Popov Conjecture. Let $n$ be an integer; by induction on $r_{X}:=$ $9-\operatorname{deg}(X)$ and by induction on $n$ we show that $\left(J_{X}\right)_{n}=\left(I_{X}\right)_{n}$. By definition, the statement is true if $n \leq 2$, for all del Pezzo surfaces $X$; if $r_{X} \leq 4$, then the result is well known for all $n$.

Suppose that $r_{X}>4, n \geq 3$, that for all del Pezzo surfaces $Y$ such that $r_{Y}<r_{X}$ we have that $J_{Y}=I_{Y}$, and that $\left(J_{X}\right)_{n-1}=\left(I_{X}\right)_{n-1}$. Let $D$ be a divisor on $X$ of anticanonical degree $n$; if there is an exceptional curve $E$ such that $D \cdot E \leq 0$, then the result follows by Lemma 9.2 or by Lemma 9.1. Otherwise $D$ is ample and the result follows from Corollary 8.5 if $\operatorname{deg}(X)=1$, or from the lemmas of Section 10 if $\operatorname{deg}(X) \geq 2$.

Quadratic generators. Let $X$ be a del Pezzo surface of degree $d$. We briefly explain how all generators of $I_{X}$ arise. The nef divisors $D$ with anticanonical degree two on $X$ are:

(1) the conics $Q$,

(2) $-K_{X}$ if $d=2$,

(3) $-K_{X}+E$ if $d=1$, where $E$ is an exceptional curve on $X$,

(4) $-2 K_{X}$ if $d=1$.

These divisors are precisely sums of pairs of intersecting exceptional curves on $X$.

We count the relations coming from conics as follows. Every conic $Q$ has $8-d$ reducible sections and $h^{0}\left(X, O_{X}(Q)\right)=2$; thus each conic gives rise to $6-d$ quadratic relations. If there are $B$ conics on $X$ then there are $(6-d) B$ generators of $I_{X}$ induced by conic bundles. For example, when $d=1$ we obtain $5 \times 2,160=$ 10,800 relations.

When $d=2$, we also have relations in degree $-K_{X}$. There are 28 monomials in $k[G]_{-K_{X}}$, and $h^{0}\left(X, \mathcal{O}_{X}\left(-K_{X}\right)\right)=3$, giving 25 linear dependence relations among these quadratic monomials. These relations yield the remaining generators of $I_{X}$.

When $d=1$, there are 30 monomials in $k[\varphi]_{-K_{X}+E}: k_{1} e, k_{2} e$ and the 28 monomials coming from the anticanonical divisor of the del Pezzo surface of degree two obtained by contracting $E$. This gives $(30-3) \times 240=6,480$ generators in $I_{X}$. Finally, we also have relations coming from $-2 K_{X}$. There are 123 monomials in $k[\mathscr{G}]_{-2 K_{X}}: k_{1}^{2}, k_{1} k_{2}, k_{2}^{2}$ and the 120 monomials of the form $e e^{\prime}$, where $E, E^{\prime} \in \mathscr{C}$ and 


\begin{tabular}{|c|c|c|c|c|c|}
\hline $\operatorname{deg}(X)$ & $D$ & $\begin{array}{c}\text { Number of } \\
\text { monomials } \\
\text { in } k\left[G^{G}\right]_{D}\end{array}$ & $h^{0}\left(X, \mathscr{O}_{X}(D)\right)$ & $b_{1, D}(\operatorname{Cox}(X))$ & $\begin{array}{c}\text { Number of } \\
\text { divisors } \\
\text { of type } D\end{array}$ \\
\hline 1 & $\begin{array}{c}Q \\
-K_{X}+E \\
-2 K_{X} \\
\text { (Total) }\end{array}$ & $\begin{array}{c}7 \\
28+2 \\
120+3 \\
22443\end{array}$ & $\begin{array}{l}2 \\
3 \\
4\end{array}$ & $\begin{array}{c}5 \\
27 \\
119 \\
17399\end{array}$ & $\begin{array}{c}2160 \\
240 \\
1 \\
2401\end{array}$ \\
\hline 2 & $\begin{array}{c}Q \\
-K_{X} \\
\text { (Total) }\end{array}$ & $\begin{array}{c}6 \\
28 \\
784\end{array}$ & $\begin{array}{l}2 \\
3\end{array}$ & $\begin{array}{c}4 \\
25 \\
529\end{array}$ & $\begin{array}{c}126 \\
1 \\
127\end{array}$ \\
\hline 3 & $\begin{array}{c}Q \\
\text { (Total) }\end{array}$ & $\begin{array}{c}5 \\
135\end{array}$ & 2 & $\begin{array}{c}3 \\
81\end{array}$ & $\begin{array}{l}27 \\
27\end{array}$ \\
\hline 4 & $\begin{array}{c}Q \\
\text { (Total) }\end{array}$ & $\begin{array}{c}4 \\
40\end{array}$ & 2 & $\begin{array}{c}2 \\
20\end{array}$ & $\begin{array}{l}10 \\
10\end{array}$ \\
\hline 5 & $\begin{array}{c}Q \\
\text { (Total) }\end{array}$ & $\begin{array}{c}3 \\
15\end{array}$ & 2 & $\begin{array}{l}1 \\
5\end{array}$ & $\begin{array}{l}5 \\
5\end{array}$ \\
\hline
\end{tabular}

Table 2. First Betti numbers $b_{1, D}(\operatorname{Cox}(X))$ for del Pezzo surfaces.

$E+E^{\prime}=-2 K_{X}$. Since $h^{0}\left(X, \mathcal{O}_{X}\left(-2 K_{X}\right)\right)=4$, we obtain 119 linear dependence relations among these quadratic monomials. These relations yield the remaining generators of $I_{X}$. This information is summarized in Table 2 .

\section{Acknowledgments}

We are grateful to Bernd Sturmfels and David Eisenbud for many profitable discussions. We thank Ragni Piene for valuable conversations on the Gauss map for curves in positive characteristic during the Spring 2009 Algebraic Geometry semester at MSRI. We would also like to thank the anonymous referee for carefully reading the manuscript and for suggesting useful improvements.

\section{References}

[Batyrev and Popov 2004] V. V. Batyrev and O. N. Popov, "The Cox ring of a del Pezzo surface", pp. 85-103 in Arithmetic of higher-dimensional algebraic varieties (Palo Alto, CA, 2002), edited by B. Poonen and Y. Tschinkel, Progr. Math. 226, Birkhäuser, Boston, MA, 2004. MR 2005h:14091 Zbl 1075.14035

[Birkar et al. 2008] C. Birkar, P. Cascini, C. D. Hacon, and J. McKernan, "Existence of minimal models for varieties of log general type", preprint, 2008. arXiv 0610203v2

[Bosma et al. 1997] W. Bosma, J. Cannon, and C. Playoust, "The Magma algebra system, I: The user language”, J. Symbolic Comput. 24:3-4 (1997), 235-265. MR 1484478 Zbl 0898.68039 
[de la Bretèche and Browning 2007] R. de la Bretèche and T. D. Browning, "On Manin's conjecture for singular del Pezzo surfaces of degree 4, I", Michigan Math. J. 55:1 (2007), 51-80. MR 2008b:14028 Zbl 1132.14019

[de la Bretèche et al. 2007] R. de la Bretèche, T. D. Browning, and U. Derenthal, "On Manin's conjecture for a certain singular cubic surface”, Ann. Sci. École Norm. Sup. (4) 40:1 (2007), 1-50. MR 2008e:11038 Zbl 1125.14008

[Brion 2007] M. Brion, "The total coordinate ring of a wonderful variety", J. Algebra 313:1 (2007), 61-99. MR 2008d:14067 Zbl 1123.14024

[Castravet and Tevelev 2006] A.-M. Castravet and J. Tevelev, "Hilbert's 14th problem and Cox rings", Compos. Math. 142:6 (2006), 1479-1498. MR 2007i:14044 Zbl 1117.14048

[Colliot-Thélène and Sansuc 1980] J.-L. Colliot-Thélène and J.-J. Sansuc, "La descente sur les variétés rationnelles", pp. 223-237 in Journées de Géometrie Algébrique d'Angers, Juillet 1979 (Alphen aan den Rijn, 1979), Sijthoff \& Noordhoff, 1980. Zbl 0451.14018

[Colliot-Thélène and Sansuc 1987] J.-L. Colliot-Thélène and J.-J. Sansuc, "La descente sur les variétés rationnelles, II”, Duke Math. J. 54:2 (1987), 375-492. MR 89f:11082 Zbl 0659.14028

[Colliot-Thélène et al. 1984] J.-L. Colliot-Thélène, J.-J. Sansuc, and P. Swinnerton-Dyer, "Intersections de deux quadriques et surfaces de Châtelet”, C. R. Acad. Sci. Paris Sér. I Math. 298:16 (1984), 377-380. MR 85j:11031 Zbl 0575.14044

[Cox 1995] D. A. Cox, "The homogeneous coordinate ring of a toric variety", J. Algebraic Geom. 4:1 (1995), 17-50. MR 95i:14046 Zbl 0846.14032

[Cragnolini and Oliverio 2000] P. Cragnolini and P. A. Oliverio, "Lines on del Pezzo surfaces with $K_{S}^{2}=1$ in characteristic 2 in the smooth case", Portugal. Math. 57:1 (2000), 59-95. MR 2001c: 14057 Zbl 0989.14012

[Debarre 2001] O. Debarre, Higher-dimensional algebraic geometry, Universitext 13, Springer, New York, 2001. MR 2002g:14001 Zbl 0978.14001

[Derenthal 2007] U. Derenthal, "Universal torsors of del Pezzo surfaces and homogeneous spaces", Adv. Math. 213:2 (2007), 849-864. MR 2008i:14052 Zbl 1125.14020

[Elizondo et al. 2004] E. J. Elizondo, K. Kurano, and K.-i. Watanabe, "The total coordinate ring of a normal projective variety”, J. Algebra 276:2 (2004), 625-637. MR 2005b:14013 Zbl 1074.14006

[Hartshorne 1977] R. Hartshorne, Algebraic geometry, Grad. Texts in Math. 52, Springer, New York, 1977. MR 57 \#3116 Zbl 0367.14001

[Hassett 2004] B. Hassett, "Equations of universal torsors and Cox rings", pp. 135-143 in Mathematisches Institut, Georg-August-Universität Göttingen: Seminars Summer Term 2004, edited by Y. Tschinkel, Universitätsdrucke Göttingen, Göttingen, 2004. MR 2007a:14046 Zbl 1108.14304

[Hassett and Tschinkel 2004] B. Hassett and Y. Tschinkel, "Universal torsors and Cox rings", pp. 149-173 in Arithmetic of higher-dimensional algebraic varieties (Palo Alto, CA, 2002), edited by B. Poonen and Y. Tschinkel, Progr. Math. 226, Birkhäuser, Boston, MA, 2004. MR 2005a:14049 Zbl 1077.14046

[Hefez and Kleiman 1985] A. Hefez and S. L. Kleiman, "Notes on the duality of projective varieties”, pp. 143-183 in Geometry today (Rome, 1984), edited by E. Arbarello et al., Progr. Math. 60, Birkhäuser, Boston, MA, 1985. MR 88f:14046 Zbl 0579.14047

[Hu and Keel 2000] Y. Hu and S. Keel, "Mori dream spaces and GIT", Michigan Math. J. 48 (2000), 331-348. MR 2001i:14059 Zbl 1077.14554

[Kaji 1992] H. Kaji, "On the inseparable degrees of the Gauss map and the projection of the conormal variety to the dual of higher order for space curves", Math. Ann. 292:3 (1992), 529-532. MR 93j:14038 Zbl 0734.14017 
[Kollár 1996] J. Kollár, Rational curves on algebraic varieties, Ergebnisse der Math. (3) 32, Springer, Berlin, 1996. MR 98c:14001 Zbl 0877.14012

[Laface and Velasco 2009] A. Laface and M. Velasco, "Picard-graded Betti numbers and the defining ideals of Cox rings", J. Algebra 322:2 (2009), 353-372. MR MR2529093

[Manin 1986] Y. I. Manin, Cubic forms, 2nd ed., North-Holland Mathematical Library 4, NorthHolland Publishing Co., Amsterdam, 1986. Algebra, geometry, arithmetic, Translated from the Russian by M. Hazewinkel. MR 87d:11037 Zbl 0582.14010

[Peyre 2004] E. Peyre, "Counting points on varieties using universal torsors", pp. 61-81 in Arithmetic of higher-dimensional algebraic varieties (Palo Alto, CA, 2002), edited by B. Poonen and Y. Tschinkel, Progr. Math. 226, Birkhäuser, Boston, MA, 2004. MR 2004m:11103

[Popov 2004] O. N. Popov, "The Cox ring of a Del Pezzo surface has rational singularities", preprint, 2004. arXiv 0402154v1

[Reid 1997] M. Reid, "Chapters on algebraic surfaces", pp. 3-159 in Complex algebraic geometry (Park City, UT, 1993), edited by J. Kollár, IAS/Park City Math. Ser. 3, Amer. Math. Soc., Providence, RI, 1997. MR 98d:14049 Zbl 0910.14016

[Salberger 1998] P. Salberger, "Tamagawa measures on universal torsors and points of bounded height on Fano varieties", pp. 91-258 in Nombre et répartition de points de hauteur bornée (Paris, 1996), vol. 251, edited by E. Peyre, 1998. MR 2000d:11091 Zbl 0959.14007

[Serganova and Skorobogatov 2007] V. V. Serganova and A. N. Skorobogatov, "Del Pezzo surfaces and representation theory", Algebra Number Theory 1:4 (2007), 393-419. MR 2009b:14070 Zbl 1170.14026

[Serganova and Skorobogatov 2008] V. Serganova and A. Skorobogatov, "On the equations for universal torsors over del Pezzo surfaces”, preprint, 2008. arXiv 0806.0089

[Stillman et al. 2007] M. Stillman, D. Testa, and M. Velasco, "Gröbner bases, monomial group actions, and the Cox rings of del Pezzo surfaces”, J. Algebra 316:2 (2007), 777-801. MR 2008i:14054

[Sturmfels and Xu 2008] B. Sturmfels and Z. Xu, "Sagbi Bases of Cox-Nagata rings", preprint, 2008. to appear in J. European Math. Soc. arXiv 0803.0892

[Terakawa 1998] H. Terakawa, "On the Kawamata-Viehweg vanishing theorem for a surface in positive characteristic", Arch. Math. (Basel) 71:5 (1998), 370-375. MR 99k:14031 Zbl 0940.14010

[Testa et al. 2009] D. Testa, A. Várilly-Alvarado, and M. Velasco, "Big rational surfaces", preprint, 2009. arXiv 0901.1094v2

[Xie 2008] Q. Xie, "Kawamata-Viehweg vanishing on rational surfaces in positive characteristic", 2008. arXiv 0710.2706v5

Communicated by Jean-Louis Colliot-Thélène

Received 2008-03-08 Revised 2009-06-05

Accepted 2009-09-14

adomani@gmail.com

Mathematical Institute, 24-29 St Giles', Oxford OX1 3LB, United Kingdom

http://www.maths.ox.ac.uk/node/7850

varilly@rice.edu

Department of Mathematics, Rice University, MS 136, Houston, TX 77005, United States http://math.rice.edu/ av15

velasco@math.berkeley.edu

Department of Mathematics, University of California, Berkeley, CA 94720, United States

http://math. berkeley.edu/ velasco 


\title{
Hilbert schemes of 8 points
}

\author{
Dustin A. Cartwright, Daniel Erman, Mauricio Velasco and Bianca Viray
}

\begin{abstract}
The Hilbert scheme $H_{n}^{d}$ of $n$ points in $\mathbb{A}^{d}$ contains an irreducible component $R_{n}^{d}$ which generically represents $n$ distinct points in $\mathbb{A}^{d}$. We show that when $n$ is at most 8 , the Hilbert scheme $H_{n}^{d}$ is reducible if and only if $n=8$ and $d \geq 4$. In the simplest case of reducibility, the component $R_{8}^{4} \subset H_{8}^{4}$ is defined by a single explicit equation, which serves as a criterion for deciding whether a given ideal is a limit of distinct points.

To understand the components of the Hilbert scheme, we study the closed subschemes of $H_{n}^{d}$ which parametrize those ideals which are homogeneous and have a fixed Hilbert function. These subschemes are a special case of multigraded Hilbert schemes, and we describe their components when the colength is at most 8 . In particular, we show that the scheme corresponding to the Hilbert function $(1,3,2,1)$ is the minimal reducible example.
\end{abstract}

\section{Introduction}

The Hilbert scheme $H_{n}^{d}$ of $n$ points in affine $d$-space parametrizes 0-dimensional, degree $n$ subschemes of $\mathbb{A}^{d}$. Equivalently, the $k$-valued points of $H_{n}^{d}$ parametrize ideals $I \subset S=k\left[x_{1}, \ldots, x_{d}\right]$ such that $S / I$ is an $n$-dimensional vector space over $k$. The smoothable component $R_{n}^{d} \subset H_{n}^{d}$ is the closure of the set of ideals of distinct points. The motivating problem of this paper is characterizing the ideals which lie in the smoothable component, that is, the 0 -dimensional subschemes which are limits of distinct points. We determine the components of the schemes $H_{n}^{d}$ for $n \leq 8$, and find explicit equations defining $R_{8}^{4} \subset H_{8}^{4}$.

We assume that $k$ is a field of characteristic not 2 or 3 .

Theorem 1.1. Suppose $n$ is at most 8 and $d$ is any positive integer. Then the Hilbert scheme $H_{n}^{d}$ is reducible if and only if $n=8$ and $d \geq 4$, in which case it consists of exactly two irreducible components: the smoothable component, of dimension $8 d$, and a component denoted $G_{8}^{d}$, of dimension $8 d-7$, which consists of local algebras isomorphic to homogeneous algebras with Hilbert function $(1,4,3)$.

MSC2000: primary 14C05; secondary 13E10.

Keywords: Hilbert scheme, zero-dimensional ideal, smoothable.

Cartwright was supported by an NSF EMSW21 fellowship. Erman is supported by an NDSEG fellowship. Velasco is partially supported by NSF grant DMS-0802851. Viray was supported by a Mentored Research Award. 
It is known that for $d$ at least 3 and $n$ sufficiently large the Hilbert scheme of points is always reducible [Iarrobino 1972]. The fact that the Hilbert scheme $H_{8}^{4}$ has at least two components appears in [Iarrobino and Emsalem 1978]. In contrast, for the Hilbert scheme of points in the plane $(d=2)$, the smoothable component is the only component [Fogarty 1968].

To show that the Hilbert scheme of $n$ points is irreducible, it suffices to show that each isomorphism type of local algebras of rank at most $n$ is smoothable, and for $n$ at most 6 there are finitely many isomorphism types of local algebras. In contrast, there are infinitely many nonisomorphic local algebras of degree 7 . Relying on a classification of the finitely many isomorphism types in degree 6, Mazzola [1980] proves the irreducibility of $H_{n}^{d}$ for $n=7$.

In our approach, a coarser geometric decomposition replaces most of the need for classification. We divide the local algebras in $H_{n}^{d}$ into sets $H_{\boldsymbol{h}}^{d}$ by their Hilbert function $\boldsymbol{h}$, and we determine which components of these sets are smoothable. The main advantage to this approach is that there are fewer Hilbert functions than isomorphism classes, and this enables us to extend the smoothability results of [Mazzola 1980] up to degree 8.

In order to determine the components of $H_{h}^{d}$, we first determine the components of the standard graded Hilbert scheme $\mathscr{H}_{h}^{d}$, which parametrizes homogeneous ideals with Hilbert function $\boldsymbol{h}$. By considering the map $\pi_{\boldsymbol{h}}: H_{\boldsymbol{h}}^{d} \rightarrow \mathscr{H}_{\boldsymbol{h}}^{d}$ which sends a local algebra to its associated graded ring, we relate the components of $\mathscr{H}_{h}^{d}$ to those of $H_{h}^{d}$. The study of standard graded Hilbert schemes leads to the following analogue of Theorem 1.1:

Theorem 1.2. Let $\mathscr{H}_{\boldsymbol{h}}^{d}$ be the standard graded Hilbert scheme for Hilbert function $\boldsymbol{h}$, where $\sum h_{i} \leq 8$. Then $\mathscr{H}_{\boldsymbol{h}}^{d}$ is reducible if and only if

$$
\boldsymbol{h}=(1,3,2,1) \quad \text { or } \quad \boldsymbol{h}=(1,4,2,1),
$$

in which case it has exactly two irreducible components. In particular, $\mathscr{H}_{(1,3,2,1)}^{3}$ is the minimal example of a reducible standard graded Hilbert scheme.

As in the ungraded case, all standard graded Hilbert schemes in the plane are smooth and irreducible [Evain 2004].

In the case when $d=4$ and $n=8$, we describe the intersection of the two components of $H_{8}^{4}$ explicitly. Let $S=k[x, y, z, w]$ and $S_{1}$ be the vector space of linear forms in $S$. Let $S_{2}^{*}$ denote the space of symmetric bilinear forms on $S_{1}$. Then, the component $G_{8}^{4}$ is isomorphic to $\mathbb{A}^{4} \times \operatorname{Gr}\left(3, S_{2}^{*}\right)$, where $\operatorname{Gr}\left(3, S_{2}^{*}\right)$ denotes the Grassmannian of 3-dimensional subspaces of $S_{2}^{*}$.

Theorem 1.3. The intersection $R_{8}^{4} \cap G_{8}^{4}$ is a prime divisor on $G_{8}^{4}$. We have the following equivalent descriptions of $R_{8}^{4} \cap G_{8}^{4} \subset G_{8}^{4}$ : 
Set-theoretic: For a point $I \in G_{8}^{4} \cong \mathbb{A}^{4} \times \operatorname{Gr}\left(3, S_{2}^{*}\right) \cong \mathbb{A}^{4} \times \operatorname{Gr}\left(7, S_{2}\right)$ let $V$ be the corresponding 7-dimensional subspace of $S_{2}$. Then $I \in G_{8}^{4}$ belongs to the intersection if and only if the skew-symmetric bilinear form

$$
\langle,\rangle_{I}:\left(S_{1} \otimes S_{2} / V\right)^{\otimes 2} \rightarrow \bigwedge^{3}\left(S_{2} / V\right) \cong k
$$

given by

$$
\left\langle l_{1} \otimes q_{1}, l_{2} \otimes q_{2}\right\rangle_{I}=\left(l_{1} l_{2}\right) \wedge q_{1} \wedge q_{2}
$$

is degenerate.

Local equations: Around any $I \in G_{8}^{4}$, choose an open neighborhood $U_{I} \subset G_{8}^{4}$ such that the universal Grassmannian bundle over the $U_{I}$ is generated by three sections. Since these sections are bilinear forms we may represent them as symmetric $4 \times 4$ matrices $A_{1}, A_{2}$, and $A_{3}$ with entries in $\Gamma\left(U_{I}, O_{G_{8}^{4}}\right)$. The local equation for $R_{8}^{4} \cap U_{I}$ is then the Pfaffian of the $12 \times 12$ matrix:

$$
\left(\begin{array}{ccc}
0 & A_{1} & -A_{2} \\
-A_{1} & 0 & A_{3} \\
A_{2} & -A_{3} & 0
\end{array}\right) .
$$

Note that specializing this equation to I gives the Pfaffian of $\langle,\rangle_{I}$.

The local equation from the previous theorem gives an effective criterion for deciding whether an algebra of colength 8 belongs to the smoothable component. Moreover, it can be lifted to equations which cut out $R_{8}^{4} \subset H_{8}^{4}$. Recall that $H_{8}^{4}$ can be covered by open affines corresponding to monomial ideals in $k[x, y, z, w]$ of colength 8 .

Theorem 1.4. On these monomial coordinate charts, $R_{8}^{4} \subset H_{8}^{4}$ is cut out settheoretically by

(1) the zero ideal on charts corresponding to monomial ideals with Hilbert functions other than $(1,4,3)$, and

(2) the pullback of the equations in Theorem 1.3 along the projection to homogeneous ideals in charts corresponding to monomial ideals with Hilbert function $(1,4,3)$.

Remark 1.5. It is not known whether $H_{8}^{4}$ is reduced. If it is, then the equations in Theorem 1.4 cut out the smoothable component scheme-theoretically.

Remark 1.6. Every point of the Hilbert scheme of $n$ points in $\mathbb{P}^{d}$ has an open neighborhood isomorphic to $H_{n}^{d}$. Therefore, analogues of Theorems 1.1, 1.3, and 1.4 hold for the Hilbert scheme of $n$ points in $\mathbb{P}^{d}$. However, the most natural setting for our methods is the affine case and the language of multigraded Hilbert schemes. 
The material in this paper is organized as follows: Section 2 contains background and definitions. Section 3 describes the geometry of standard graded Hilbert schemes of degree at most 8 . Section 4 contains proofs of the smoothability of families of algebras and its main steps are collected in Table 4.1. Section 5 is devoted to the study of the components of $H_{8}^{4}$ and their intersection. Section 6 ties together these results to give proofs of all theorems mentioned above. Finally, Section 7 proposes some open questions.

\section{Background}

In this section, let $k$ be a field and $S=k\left[x_{1}, \ldots, x_{d}\right]$.

Multigraded Hilbert schemes. A grading of $S$ by an abelian group $A$ is a semigroup homomorphism deg: $\mathbb{N}^{d} \rightarrow A$ which assigns to each monomial in $S$ a degree in $A$. Let $h: A \rightarrow \mathbb{N}$ be an arbitrary function, which we will think of as a vector $\boldsymbol{h}$, with values $h_{a}$ indexed by $a$ in $A$. We say that a homogeneous ideal $I$ in $S$ has Hilbert function $\boldsymbol{h}$ if $S_{a} / I_{a}$ has $k$-dimension $h_{a}$ for all $a \in A$. Multigraded Hilbert schemes, introduced in [Haiman and Sturmfels 2004], parametrize homogeneous ideals with a fixed Hilbert function. More precisely these are quasiprojective schemes over $k$ which represent the following functors [Haiman and Sturmfels 2004, Theorem 1.1]:

Definition 2.1. For a fixed integer $d$, grading deg, and Hilbert function $\boldsymbol{h}$, the

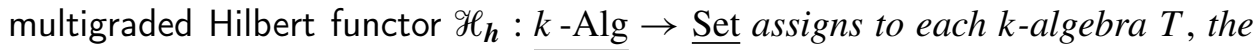

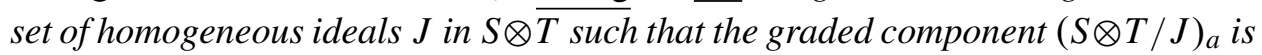
a locally free $T$-module of rank $h_{a}$ for all $a$ in $A$. The multigraded Hilbert scheme is the scheme which represents the multigraded Hilbert functor.

In particular, we will be interested in the following two special kinds of multigraded Hilbert scheme:

- Let deg : $\mathbb{N}^{d} \rightarrow 0$ be the constant function to the trivial group and define $h_{0}=n$. In this case the multigraded Hilbert scheme is the Hilbert scheme of $n$ points in $\mathbb{A}^{d}$ and will be denoted $H_{n}^{d}$.

- Let $\operatorname{deg}: \mathbb{N}^{d} \rightarrow \mathbb{Z}$ be the summation function, which induces the standard grading $\operatorname{deg}\left(x_{i}\right)=1$. We call the corresponding multigraded Hilbert scheme the standard graded Hilbert scheme for Hilbert function $\boldsymbol{h}$ and denote it with $\mathcal{H}_{\boldsymbol{h}}^{d}$.

If $n=\sum_{j \in \mathbb{N}} h_{j}$ there is a closed immersion $\mathscr{H}_{h}^{d} \rightarrow H_{n}^{d}$ by [Haiman and Sturmfels 2004, Proposition 1.5].

Coordinates for the Hilbert scheme of points. In this section we briefly discuss some coordinate systems on $H_{n}^{d}$. The reader should refer to [Miller and Sturmfels 
2005, Chapter 18] for an extended treatment. For a monomial ideal $M$ of colength $n$ with standard monomials $\lambda$, let $U_{\lambda} \subset H_{n}^{d}$ be the set of ideals $I$ such that the monomials in $\lambda$ are a basis for $S / I$. Note that the $U_{\lambda}$ form an open cover of $H_{n}^{d}$. An ideal $I \in U_{\lambda}$ has generators of the form $m-\sum_{m^{\prime} \in \lambda} c_{m^{\prime}}^{m} m^{\prime}$. The $c_{m^{\prime}}^{m}$ are local coordinates for $U_{\lambda}$ which define a closed immersion into affine space.

Suppose $V(I)$ consists of $n$ distinct points $q^{(1)}, \ldots, q^{(n)}$ with coordinates $q_{i}^{(j)}$ for $1 \leq i \leq d$. Fix an order $\lambda=\left(m_{1}, \ldots, m_{n}\right)$ on the set of monomials $\lambda$ and define $\Delta_{\lambda}=\operatorname{det}\left(\left[m_{i}\left(q^{(j)}\right)\right]_{i, j}\right)$. For example, if $\lambda=\left(1, x_{1}, \ldots, x_{1}^{n-1}\right)$, then $\Delta_{\lambda}$ is the determinant of the Vandermonde matrix on the $q_{1}^{(j)}$. If $I \in U_{\lambda}$, we can express the $c_{m^{\prime}}^{m}$ in terms of the $q_{i}^{(j)}$, using Cramer's rule, as

$$
c_{m^{\prime}}^{m}=\frac{\Delta_{\lambda-m^{\prime}+m}}{\Delta_{\lambda}},
$$

where $\lambda-m^{\prime}+m$ is the ordered set of monomials obtained from $\lambda$ by replacing $m^{\prime}$ with $m$. Note that the right-hand side of this equality is only defined for ideals of distinct points. The quotient or product of two $\Delta_{\lambda}$ 's is $S_{n}$-invariant. Thus the formula does not depend on the order of $\lambda$. Gluing over the various $U_{\lambda}$, these quotients determine a birational map

$$
\left(\mathbb{A}^{d}\right)^{n} / / S_{n} \rightarrow R_{n}^{d}
$$

that is regular when the points $q^{(j)}$ are all distinct. The rational functions $\Delta_{\eta_{1}} / \Delta_{\eta_{2}}$ are elements of the quotient field of either $\left(\mathbb{A}^{d}\right)^{n} / / S_{n}$ or $R_{n}^{d}$. The expressions $\Delta_{\lambda}$ and their relationship to the local equations $c_{m^{\prime}}^{m}$ were introduced in [Haiman 1998, Proposition 2.6].

Duality. First let us suppose that $k$ has characteristic 0 , and let $S^{*}$ be the ring $k\left[y_{1}, \ldots, y_{d}\right]$, with the structure of an $S$-module via formal partial differentiation $x_{i} \cdot f=\partial f / \partial y_{i}$. If we look at homogeneous polynomials of a fixed degree $j$ in each of the two rings, we have a pairing $S_{j} \times S_{j}^{*} \rightarrow S_{0}^{*}=k$. Any vector subspace of $S_{j}$ has an orthogonal subspace in $S_{j}^{*}$ of complementary dimension. In particular, if $I$ is a homogeneous ideal in $S$, we have subspaces $I_{j}^{\perp} \subset S_{j}^{*}$ and we set $I^{\perp}=\oplus I_{j}^{\perp}$, which is often called the Macaulay inverse system of $I$. The subspace $I^{\perp}$ is closed under differentiation, that is, $\frac{\partial}{\partial y_{i}} I_{j}^{\perp} \subset I_{j-1}^{\perp}$ for all $i$ and $j$. Conversely, any graded vector subspace $I^{\perp} \subset S^{*}$ which is closed under differentiation determines an orthogonal ideal $I \subset S$ with Hilbert function $h_{j}=\operatorname{dim}_{k} I_{j}^{\perp}$. Also, note that any linear change of variables in $S$ induces a linear change of variables in $S^{*}$.

If $k$ has positive characteristic $p$, then the same theory works for sufficiently small degree. Formal partial differentiation gives a perfect pairing $S_{j} \times S_{j}^{*} \rightarrow k$ if and only if $j$ is less than $p$. Thus, we can associate orthogonal subspaces $I_{j}^{\perp}$ to a homogeneous ideal $I$ so long as $I_{p}$ contains all of $S_{p}$. In this case, we define 
$I_{j}^{\perp}=0$ for all degrees $j$ at least $p$, and $I=\oplus I_{j}$ as before. Conversely, for a graded vector subspace $I^{\perp} \subset S_{*}$ which is closed under differentiation and with $I_{j}^{\perp}=0$ for $j$ at least $p$, the orthogonal space is a homogeneous ideal $I \subset S$ with Hilbert function $h_{j}=\operatorname{dim}_{k} I_{j}^{\perp}$.

\section{Components of the standard graded Hilbert schemes}

In this section, $k$ will denote an algebraically closed field of characteristic not 2 or 3.

We will study the components of the standard graded Hilbert schemes $\mathscr{H}_{\boldsymbol{h}}^{d}$ with Hilbert function $\boldsymbol{h}$ where $\sum h_{i} \leq 8$. These results will be important for the proofs of smoothability in the following section. From [Evain 2004, Theorem 1], we have that for $d=2$, the standard graded Hilbert schemes are irreducible. Thus, we will only work with $d$ at least 3 . For the purposes of classifying irreducible components of $\mathcal{H}_{\boldsymbol{h}}^{d}$, it is convenient to work with homogeneous ideals which contain no linear forms, and thus we assume that $h_{1}=d$. The following lemma allows us to restrict our attention to this case:

Proposition 3.1. The standard graded Hilbert scheme $\mathscr{H}_{\boldsymbol{h}}^{d}$ with $d \geq h_{1}$ is a $\mathcal{H}_{\boldsymbol{h}}^{h_{1}}$ bundle over $\operatorname{Gr}\left(d-h_{1}, S_{1}\right)$. In particular, if $\mathcal{H}_{\boldsymbol{h}}^{h_{1}}$ is irreducible of dimension $D$ then $\mathcal{H}_{\boldsymbol{h}}^{d}$ is irreducible of dimension $D+\left(d-h_{1}\right) d$.

Proof. The degree 1 summand of the universal ideal sheaf of $\mathcal{O}_{\mathscr{H e}_{h}^{d}}\left[x_{1}, \ldots, x_{d}\right]$ is locally free of rank $d-h_{1}$ and thus defines a morphism $\phi: \mathscr{H}_{\boldsymbol{h}}^{d} \rightarrow \operatorname{Gr}\left(d-h_{1}, S_{1}\right)$. Over an open affine $U \cong \mathbb{A}^{\left(d-h_{1}\right) h_{1}}$ in $\operatorname{Gr}\left(d-h_{1}, S_{1}\right)$, we have an isomorphism $\phi^{-1}(U) \cong U \times H_{\boldsymbol{h}}^{h_{1}}$ by taking a change of variables in $\mathrm{O}_{U}\left[x_{1}, \ldots, x_{d}\right]$.

Lemma 3.2. Let be $m$ a positive integer such that $m$ ! is not divisible by the characteristic of $k$. Let $f\left(y_{1}, \ldots, y_{d}\right)$ be a homogeneous polynomial in $S_{m}^{*}$ whose partial derivatives form an $r$-dimensional vector subspace of $S_{m-1}^{*}$. Then $f$ can be written as a polynomial in terms of some $r$-dimensional subspace of $S_{1}^{*}$.

Proof. There exists a linear map from $S_{1} \rightarrow S_{m-1}^{*}$ which sends $x_{i} \mapsto \frac{\partial f}{\partial y_{i}}$. After a change of variables, we can assume that $x_{r+1}, \ldots, x_{d}$ annihilate $f$. Thus, any term of $f$ contains only the variables $y_{1}, \ldots, y_{r}$.

Throughout this section, $N$ will denote $\operatorname{dim}_{k} S_{2}=\left(\begin{array}{c}d+1 \\ 2\end{array}\right)$, the dimension of the vector space of quadrics.

Proposition 3.3. Let $0 \leq e \leq N$. The standard graded Hilbert scheme for Hilbert function $(1, d, e)$ is isomorphic to the Grassmannian $\operatorname{Gr}\left(N-e, S_{2}\right)$, and it is thus irreducible of dimension $(N-e) N$.

Proof. We build the isomorphism via the functors of points of these schemes. For a $k$-algebra $T$ let $\phi(T): \mathscr{H}_{h}(T) \rightarrow \operatorname{Gr}\left(N-e, S_{2}\right)(T)$ be the morphism of sets which 
maps a homogeneous ideal $I \subset T \otimes_{k} S$ to $I_{2}$. Let $\psi(T): \operatorname{Gr}\left(N-e, S_{2}\right)(T) \rightarrow \mathscr{H}_{h}(T)$ be the map which sends a $k$-submodule $L$ of $T \otimes S_{2}$ to $L \oplus \bigoplus_{j \geq 3}\left(T \otimes_{k} S_{j}\right)$, which is an ideal of $T \otimes S$. The natural transformations $\phi$ and $\psi$ are inverses of one another and the isomorphism follows from Yoneda's Lemma.

Proposition 3.4. Let $\boldsymbol{h}=(1, d, 1, \ldots, 1)$ and let $m \geq 3$ be the largest index such that $h_{m}$ is nonzero. Then the standard graded Hilbert scheme for $\boldsymbol{h}$ is irreducible of dimension $d-1$.

Proof. We claim that the scheme $\mathscr{H}_{\boldsymbol{h}}^{d}$ is parametrized by $\operatorname{Gr}\left(1, S_{1}^{*}\right)$ by sending a vector space generated by $\ell \in S_{1}^{*}$ to the ideal generated by the quadrics orthogonal to $\ell^{2}$ and all degree $m+1$ polynomials. This ideal has the right Hilbert function and the parametrization is clearly surjective.

Theorem 3.5. If $d$ is at least 3 , the standard graded Hilbert scheme for Hilbert function $(1, d, 2,1)$ is reducible and consists of the following two components:

(1) The homogeneous ideals orthogonal to $\ell^{3}, \ell^{2}$, and $q$ where $\ell$ is a linear form and $q$ is a quadric linearly independent of $\ell^{2}$. We denote this component by $2_{d}$, and $\operatorname{dim}\left(2_{d}\right)=\left(d^{2}+3 d-6\right) / 2$.

(2) The closure of the homogeneous ideals orthogonal to a cubic $c$ and its partial derivatives, where the degree 1 derivatives of $c$ have rank 2 . We denote this component by $\mathscr{P}_{d}$, and $\operatorname{dim}\left(\mathscr{P}_{d}\right)=2 d-1$.

Proof. We compute the dimension of the first component. It is parametrized by the 1-dimensional subspace of $S_{1}^{*}$ generated by $\ell$ and a 2-dimensional subspace of $S_{2}^{*}$ which contains $\ell^{2}$. These have dimensions $d-1$ and $N-1-1$ respectively, for a total of $\left(d^{2}+3 d-6\right) / 2$.

Note also that an open subset of the second component, $\mathscr{P}_{d}$, is parametrized by a 2-dimensional subspace $V$ of $S_{1}^{*}$ and a cubic $c \in \operatorname{Sym}_{3}(V)$ which is not a perfect cube. The parametrization is by taking the ideal whose components of degrees 3 and 2 are orthogonal to $c$ and to its derivatives respectively. The space of derivatives is 2-dimensional by our construction of $c$. The dimension of $\mathscr{P}_{d}$ is $3+2(d-2)=2 d-1$.

We claim that any homogeneous ideal with Hilbert function $(1, d, 2,1)$ lies in one of these two components. Any such ideal is orthogonal to a cubic $c$, and the derivatives of $c$ are at most 2-dimensional. If the derivatives are 1-dimensional, then $c$ must be a perfect cube, so the ideal is in $\mathscr{2}_{d}$. Otherwise, the ideal is in $\mathscr{P}_{d}$.

Finally, we will show that $\mathscr{P}_{d}$ has a point that does not lie on $\mathscr{2}_{d}$. Let $I$ be the ideal orthogonal to $y_{1} y_{2}^{2}$, and its partial derivatives, $2 y_{1} y_{2}, y_{2}^{2}$. Then $I$ is generated by $x_{2}^{3}$ and all degree 2 monomials other than $x_{2}^{2}$ and $x_{1} x_{2}$. We will study the homomorphisms $\phi: I \rightarrow S / I$ of degree 0 , as these correspond to the tangent space of $\mathcal{H}_{\boldsymbol{h}}^{d}$ at $I$. For any quadric generator $q$, we can write $\phi(q)=a_{q} x_{2}^{2}+b_{q} x_{1} x_{2}$. Note 
that $x_{1} \phi(q)=a_{q} x_{1} x_{2}^{2}$ and $x_{2} \phi(q)=b_{q} x_{1} x_{2}^{2}$. For any $i, j>2, \phi$ must satisfy the conditions

$$
\begin{array}{cl}
x_{1} \phi\left(x_{i} x_{j}\right)=x_{j} \phi\left(x_{i} x_{1}\right)=0, & x_{2} \phi\left(x_{i} x_{j}\right)=x_{j} \phi\left(x_{i} x_{2}\right)=0, \\
x_{1} \phi\left(x_{1} x_{i}\right)=x_{i} \phi\left(x_{1}^{2}\right)=0, & x_{2} \phi\left(x_{1} x_{i}\right)=x_{1} \phi\left(x_{2} x_{i}\right) .
\end{array}
$$

In matrix form, we see that $\phi$ must be in the form

$$
\begin{aligned}
& x_{2}^{2} \\
& x_{1} x_{2} \\
& x_{1} x_{2}^{2}
\end{aligned}\left(\begin{array}{ccccc}
* & x_{1} x_{i} & x_{2} x_{i} & x_{i} x_{j} & x_{2}^{3} \\
* & 0 & c_{i} & 0 & 0 \\
0 & 0 & 0 & 0 & 0 \\
c_{i} & 0 & 0
\end{array}\right)
$$

where $i$ and $j$ range over all integers greater than 2 . Thus there are at most $2(d-2)+3=2 d-1$ tangent directions, but since $\mathscr{P}_{d}$ has dimension $2 d-1$, there are exactly $2 d-1$ tangent directions. On the other hand, $2_{d}$ has dimension $\left(d^{2}+3 d-6\right) / 2$, which is greater than $2 d-1$ for $d$ at most 3 , so $I$ cannot belong to $\mathscr{2}_{d}$ and thus $\mathscr{P}_{d}$ is a component.

Proposition 3.6. The standard graded Hilbert scheme for Hilbert function $\boldsymbol{h}=$ $(1, d, 2,2)$ is irreducible of dimension $2 d-2$.

Proof. The Hilbert scheme is parametrized by a 2-dimensional subspace $L$ of $S_{1}^{*}$ and a subspace of $S_{2}$ of dimension $N-2$, and containing the $(N-3)$-dimensional subspace orthogonal to the square of $L$. The parametrization is by sending the subspace of $S_{2}$ to the ideal generated by that subspace, together with all degree 4 polynomials. The dimension of this parametrization is $2(d-2)+2=2 d-2$.

Proposition 3.7. The standard graded Hilbert scheme for Hilbert function $\boldsymbol{h}=$ $(1,3,3,1)$ is irreducible of dimension 9 .

Proof. This Hilbert scheme embeds as a closed subscheme of the smooth 18dimensional variety $\operatorname{Gr}\left(3, S_{2}\right) \times \operatorname{Gr}\left(9, S_{3}\right)$ by mapping an ideal to its degree 2 and 3 graded components. Furthermore, $\mathscr{H}_{\boldsymbol{h}}^{3}$ is defined by $9=3 \cdot 3$ equations, corresponding to the restrictions that the products of each of the 3 variables and each of the 3 quadrics in $I_{2}$ are in $I_{3}$. In particular, the dimension of each irreducible component is at least 9 .

Now we will look at the projection of $\mathscr{H}_{\boldsymbol{h}}^{3}$ onto the Grassmannian $\operatorname{Gr}\left(9, S_{3}\right)$, which is isomorphic to $\operatorname{Gr}\left(1, S_{3}^{*}\right)$. The orthogonal cubic in $S_{3}^{*}$ can be classified according to the vector space dimension of its derivatives. For a generic cubic, its three derivatives will be linearly independent and therefore the cubic will completely determine the orthogonal space. Thus, the projection from $\mathscr{H}_{\boldsymbol{h}}^{3}$ is a bijection over this open set, so the preimage is 9-dimensional. In the case where the derivatives of the cubic are 2-dimensional, we have that, after a change of coordinates, the 
cubic is written in terms of two variables. Thus, the parameter space of the cubic consists of a 2-dimensional choice of a subspace of $S_{1}$ and then a 3-dimensional choice of a cubic written in terms of this subspace. The fiber over any fixed cubic is isomorphic to the Grassmannian of 3-dimensional subspaces of the 4-dimensional subspace of $S_{2}$ orthogonal to the derivatives of the cubic. The dimension of the locus in $\mathscr{H}_{\boldsymbol{h}}^{3}$ is therefore $2+3+3=8$. By a similar logic, the locus where the cubic has a 1 -dimensional space of derivatives is $2+2 \cdot 3=8$. Therefore, $\mathscr{H}_{\boldsymbol{h}}^{3}$ is the disjoint union of three irreducible sets, of dimensions 9,8 , and 8 . We conclude that $\mathcal{H}_{\boldsymbol{h}}^{3}$ is an irreducible complete intersection of dimension 9.

Proposition 3.8. Let $1 \leq e \leq N$. The standard graded Hilbert scheme for Hilbert function $(1, d, e, 1,1)$ is irreducible of dimension $d-1+(N-e)(e-1)$.

Proof. This Hilbert scheme is parametrized by a 1-dimensional subspace $L$ of $S_{1}^{*}$, together with an $e$-dimensional subspace $V$ of $S_{2}^{*}$ which contains $\operatorname{Sym}_{2}(L)$. The parametrization is by mapping $(L, V)$ to the ideal whose summands of degrees 2,3 , and 4 are orthogonal to $V, L^{3}$, and $L^{4}$, respectively. Note that this has the desired dimension $(d-1)+((N-1)-(e-1))(e-1)$.

Theorem 3.9. With the exception of Hilbert functions $(1,3,2,1)$ and $(1,4,2,1)$, the standard graded Hilbert schemes with $\sum h_{i} \leq 8$ are irreducible.

Proof. The cases when $d=2$ follow from [Evain 2004, Theorem 1]. The cases when $d$ is at least 3 are summarized in Table 4.1.

\section{Smoothable 0 -schemes of degree at most 8}

In this section $k$ will denote an algebraically closed field of characteristic not 2 or 3 .

Recall that a point $I$ in $H_{n}^{d}$ is smoothable if $I$ belongs to the smoothable component $R_{n}^{d}$. In this section, we first reduce the question of smoothability to ideals $I$ in $H_{n}^{d}$ where $S / I$ is a local $k$-algebra and $I$ has embedding dimension $d$. Then we define the schemes $H_{\boldsymbol{h}}^{d}$ which parametrize local algebras, and we use these to show that each 0 -dimensional algebra of degree at most 8 is either smoothable or is isomorphic to a homogeneous local algebra with Hilbert function $(1,4,3)$.

We use two different methods to show that a subscheme $H_{h}^{d}$ belongs to the smoothable component.

(1) For each irreducible component of $H_{h}^{d}$, consider a generic ideal $I$ from that component. Apply suitable isomorphisms to put $I$ into a nice form. Then show $I$ is smoothable. Since the set of ideals isomorphic to $I$ are dense in the component and smoothable, the entire component of $H_{\boldsymbol{h}}^{d}$ containing $I$ must belong to $R_{n}^{d}$. 
(2) Within each irreducible component of $H_{h}^{d}$, find an ideal $I$ such that $I$ is a smooth point in $H_{n}^{d}$ and $I$ belongs to $R_{n}^{d}$. Then the whole component of $H_{h}^{d}$ containing $I$ must belong to $R_{n}^{d}$.

In each method we need to show that a particular ideal $I$ is smoothable. We do this by showing $I=$ in $J$ with respect to some nonnegative weight vector for a smoothable ideal $J$. The corresponding Gröbner deformation induces a morphism $\mathbb{A}^{1} \rightarrow R_{n}^{d}$ which maps 0 to $I$.

For $d=2$, the Hilbert scheme $H_{n}^{2}$ is smooth and irreducible [Fogarty 1968, Theorem 2.4]. Thus, we will limit our analysis to algebras with embedding dimension at least 3.

For a finite rank $k$-algebra $A_{0}$, we say that $A_{0}$ is smoothable if there exists a flat family $k \llbracket t \rrbracket \rightarrow A$ such that the special fiber of $A$ is isomorphic to $A_{0}$ and such that the generic fiber of $A$ is smooth. This terminology is justified by the following result:

Lemma 4.1. Let $I \subseteq S$ an ideal of colength $n$. Then $I$ is smoothable if and only if $S / I$ is smoothable (as a k-algebra).

Proof. Let $I \subseteq S$ be a smoothable ideal, and let $U \subseteq R_{n}^{d}$ be the open set parametrizing smooth 0 -schemes. Since $I \in R_{n}^{d}$, there exists a smooth curve $C$ and a map $f: C \rightarrow R_{n}^{d}$ such that $f(P)=I$ for some point $P \in C$ and such that $f(C)$ meets $U$. By considering the completion of $C$ at the point $P$, we obtain an induced map $\widehat{f}: \operatorname{Spec}(k \llbracket t \rrbracket) \rightarrow R_{n}^{d}$ which sends the closed point to $I$ and the generic point into $U$. The flat family over $\operatorname{Spec}(k \llbracket t \rrbracket)$ which corresponds to the map $\widehat{f}$ induces an abstract smoothing of $S / I$.

Conversely, let's assume that $A_{0}:=S / I$ admits an abstract smoothing $k \llbracket t \rrbracket \rightarrow A$. Let $\phi: k \llbracket t \rrbracket\left[x_{1}, \ldots, x_{d}\right] \rightarrow A$ be defined by sending each $x_{i}$ to any lift of $x_{i}$ from $S / I=A_{0}$. Since $A$ is a finitely generated $k \llbracket t \rrbracket$-module, the cokernel of $\phi$ is finitely generated, and so we can apply Nakayama's Lemma to show that $\phi$ is surjective. The map $\phi$ thus induces a morphism of schemes $f: \operatorname{Spec}(k \llbracket t \rrbracket) \rightarrow H_{n}^{d}$ which sends the closed point to $I$ and the generic point into $U$. It follows that $I \in \bar{U}=R_{n}^{d}$.

If $I$ is supported at multiple points, then $S / I$ is the product of local Artin algebras and a smoothing of each factor over $k \llbracket t \rrbracket$ yields a smoothing of $S / I$. Because of this observation and Lemma 4.1, we will now only consider ideals $I$ in $H_{n}^{d}$ which define local algebras with embedding dimension $d$.

The schemes $\boldsymbol{H}_{\boldsymbol{h}}^{\boldsymbol{d}}$. If $(A, \mathfrak{m})$ is a local algebra, its Hilbert function is defined by $h_{i}=\operatorname{dim}_{k} \mathfrak{m}^{i} / \mathfrak{m}^{i+1}$, which is equivalently the Hilbert function of the associated graded ring of $A$. When $A$ is both local and graded, the two notions of Hilbert function coincide. We now define the schemes $H_{h}^{d}$ and explore their irreducible components for each Hilbert function $\boldsymbol{h}$ with $\sum h_{i} \leq 8$. 
For each $\boldsymbol{h}$ such that $\sum h_{i}=n$, the subscheme $H_{\boldsymbol{h}}^{d} \subset H_{n}^{d}$ consists set-theoretically of the ideals $I$ defining a local algebra $S / I$ with maximal ideal $\left(x_{1}, \ldots, x_{d}\right)$ whose Hilbert function equals $\boldsymbol{h}$. More precisely, let $\mathscr{A}={O^{\prime}}_{n}\left[x_{1}, \ldots, x_{d}\right] / \mathscr{I}$ be the universal sheaf of algebras on $H_{n}^{d}$ and let $\mathcal{M}$ be the ideal $\left(x_{1}, \ldots, x_{d}\right) \mathscr{A}$. The fiber at an ideal $I$ of the quotient sheaf $\mathscr{A} / \mathcal{M}^{i}$ is isomorphic to $S /\left(I+\left(x_{1}, \ldots, x_{d}\right)^{i}\right)$. For any fixed $\boldsymbol{h}$, there is a locally closed subset of $H_{n}^{d}$ consisting of those points such that the fiber of $\mathscr{A} / M^{i}$ has dimension $h_{0}+\cdots+h_{i-1}$ for all $i \geq 0$. Let $H_{\boldsymbol{h}}^{d}$ be the reduced subscheme on this subset, and then the restriction of each $\mathscr{A} / \mathcal{M}^{i}$ to $H_{\boldsymbol{h}}^{d}$ is locally free. Define $\mathscr{B}$ to be the sheaf of graded algebras on $H_{\boldsymbol{h}}^{d}$ whose $i$-th component is

$$
\operatorname{ker}\left(\left.\left.\left(\mathscr{A} / \mathcal{M}^{i+1}\right)\right|_{H_{h}^{d}} \rightarrow\left(\mathscr{A} / \mathcal{M}^{i}\right)\right|_{H_{h}^{d}}\right),
$$

which is locally free of rank $h_{i}$ because it is the kernel of a surjection of locally free sheaves. Note that the fiber of $\mathscr{B}$ at $I$ is the associated graded ring of $S / I$. There is a canonical surjection of graded algebras $O_{H_{h}^{d}}\left[x_{1}, \ldots, x_{d}\right] \rightarrow \mathscr{B}$ which defines a morphism $\pi_{\boldsymbol{h}}: H_{\boldsymbol{h}}^{d} \rightarrow \mathcal{H}_{\boldsymbol{h}}^{d}$ to the standard graded Hilbert scheme. The ideal $I$ gets mapped to its initial ideal with respect to the weight vector $(-1, \ldots,-1)$.

With the exception $\boldsymbol{h}=(1,3,2,1,1)$, we will show that the irreducible components of $H_{\boldsymbol{h}}^{d}$ and $\mathscr{H}_{\boldsymbol{h}}^{d}$ are in bijection via the map $\pi_{\boldsymbol{h}}$.

Proposition 4.2. Each subscheme $H_{(1, d, e)}^{d}$ is irreducible.

Proof. Since $H_{(1, d, e)}^{d} \cong \mathscr{H}_{(1, d, e)}^{d}$, this follows from Proposition 3.3.

Proposition 4.3. Fix $\boldsymbol{h}=(1, d, e, f)$. Let $m=(d+1) d / 2-e=\operatorname{dim}_{k} S_{2} / I_{2}$. Then every fiber of $\pi_{\boldsymbol{h}}$ is irreducible of dimension $\mathrm{mf}$. In particular, the irreducible components of $H_{\boldsymbol{h}}^{d}$ are exactly the preimages of the irreducible components of $\mathscr{H}_{\boldsymbol{h}}^{d}$.

Proof. Fix a point in $\mathscr{H}_{\boldsymbol{h}}^{d}$, which corresponds to a homogeneous ideal $I$. Let $q_{1}, \ldots, q_{m}$ be quadratic generators of $I$, and let $c_{1}, \ldots, c_{f}$ be cubics which form a vector space basis for $S_{3} / I_{3}$. Define a map $\phi: \mathbb{A}^{m f} \rightarrow H_{n}^{d}$ via the ideal

$$
\left\langle q_{i}-\sum_{j=1}^{f} t_{i j} c_{j} \mid 1 \leq i \leq m\right\rangle+I_{\geq 3}
$$

where the $t_{i j}$ are the coordinate functions of $\mathbb{A}^{m f}$. Because a product of any variable $x_{\ell}$ with any of these generators is in $I$, this ideal has the right Hilbert function and maps to the fiber of $\pi_{\boldsymbol{h}}$ over $I$. Furthermore, $\phi$ is bijective on field-valued points, so the fiber is irreducible of dimension $m f$.

For the last statement, we have that for any irreducible component of $\mathscr{H}_{\boldsymbol{h}}^{d}$, the restriction of $\pi_{\boldsymbol{h}}$ has irreducible equidimensional fibers over an irreducible base, so the preimage is irreducible. These closed sets cover $H_{h}^{d}$ and because each lies over a distinct component of $\mathscr{H}_{\boldsymbol{h}}^{d}$, they are distinct irreducible components. 
Combining Theorem 3.5 with the above proposition, we see $H_{(1, d, 2,1)}^{d}$ has exactly two components: $P_{d}:=\pi^{-1}\left(\mathscr{P}_{d}\right)$ and $Q_{d}:=\pi^{-1}\left(\mathscr{Q}_{d}\right)$. In addition, by Propositions 3.6, 3.7, and 4.3, $H_{(1, d, 2,2)}^{d}$ and $H_{(1,3,3,1)}^{3}$ are irreducible.

Proposition 4.4. Let $\boldsymbol{h}=(1, d, 1, \ldots, 1)$ and let $m \geq 3$ be the largest index such that $h_{m}$ is nonzero. Then $H_{h}^{d}$ is irreducible of dimension $(d+2 m-2)(d-1) / 2$. At a generic point, after a change of coordinates, we can take the ideal to be

$$
\left\langle x_{d}^{m+1}, x_{i}^{2}-x_{d}^{m}, x_{j} x_{k} \mid 1 \leq i<d, 1 \leq j<k \leq d\right\rangle .
$$

Proof. Fix an ideal $I \in \mathscr{H}_{\boldsymbol{h}}^{d}$, and after a change of coordinates, we can assume

$$
I=\left\langle x_{d}^{m+1}, x_{i} x_{j} \mid 1 \leq i \leq d-1,1 \leq j \leq d\right\rangle .
$$

Let $J$ be an ideal in the fiber above $I$. By assumption $J$ contains an element of the form

$$
x_{i} x_{d}-b_{i 3} x_{d}^{3}-\cdots-b_{i m} x_{d}^{m}
$$

for $1 \leq i<d$. Let $J^{\prime}$ be the image of $J$ after the change of coordinates

$$
x_{i} \mapsto x_{i}+b_{i 3} x_{d}^{2}+\cdots b_{i m} x_{d}^{m-1},
$$

and note that $J^{\prime}$ contains $x_{i} x_{d}$ for $1 \leq i<d$ and also lies over $I$. Thus, for each $1 \leq i \leq j<d, J^{\prime}$ contains an element of the form $f=x_{i} x_{j}-a_{i j} x_{d}^{k}-\cdots$ for some $k$. However, $J^{\prime}$ must also contain $x_{j}\left(x_{i} x_{d}\right)-x_{d} f=a_{i j} x_{d}^{k+1}+\cdots$, so in order to have $I$ as the initial ideal, $k$ must equal $m$. Therefore, $J^{\prime}$ is of the form

$$
J^{\prime}=\left\langle x_{d}^{m+1}, x_{i} x_{j}-a_{i j} x_{d}^{m}, x_{k} x_{d} \mid 1 \leq i \leq j \leq d, 1 \leq k<d\right\rangle .
$$

Conversely, for any choice of $a_{i j}$ and $b_{i j}$, applying the change of variables in (4-1) to the ideal $J^{\prime}$ gives a unique ideal $J$ with $I$ as an initial ideal. Thus, the fiber is irreducible of dimension $(m-2)(d-1)+(d-1) d / 2=(d-1)(d+2 m-4) / 2$, which, together with Proposition 3.4 proves the first statement.

For the second statement, note that the coefficients $a_{i j}$ define a symmetric bilinear form. By taking the form to be generic and choosing a change of variables, we get the desired presentation of the quotient algebra.

The above propositions cover all Hilbert functions of length at most 8 except for $\boldsymbol{h}=(1,3,2,1,1)$. In this case the fibers of $\pi_{(1,3,2,1,1)}$ are not equidimensional. The dimension of the fiber depends on whether or not the homogeneous ideal requires a cubic generator.

Lemma 4.5. No ideal in $\mathscr{H}_{(1,3,2,1,1)}^{3}$ requires a quartic generator.

Proof. If $I$ were such an ideal, then leaving out the quartic generator would yield an ideal with Hilbert function $(1,3,2,1,2)$. No such ideal exists, because no such monomial ideal exists. 
Lemma 4.6. There exists a 4-dimensional irreducible closed subset $\mathscr{L}$ of $\mathscr{H}=$ $\mathscr{H}_{(1,3,2,1,1)}^{3}$ where the corresponding homogeneous ideal requires a single cubic generator. $O n \mathcal{U}=\mathscr{H} \backslash \mathscr{L}$, the ideal does not require any cubic generators.

Proof. Let $\mathscr{Y}_{j}$ denote the $j$-th graded component of $\mathscr{O}_{\mathscr{H}}[x, y, z]$ and $\mathscr{I}_{j} \subset \mathscr{Y}_{j}$ the $j$ th graded component of the universal family of ideals on $\mathscr{H}$. Consider the cokernel 2 of the multiplication map on the coherent sheaves $\mathscr{I}_{2} \otimes_{\mathcal{O H}} \mathscr{I}_{1} \rightarrow \mathscr{I}_{3}$ on $\mathscr{H}$. The dimension of 2 is upper semicontinuous. Furthermore, since it is not possible to have an algebra with Hilbert function $(1,3,2,3)$, the dimension is at most 1 . The set $\mathscr{E}$ is exactly the support of $\mathscr{2}$.

We claim that $\mathscr{L}$ is parametrized by the data of a complete flag $V_{1} \subset V_{2} \subset S_{1}^{*}$, and a 2-dimensional subspace $Q$ of $V_{2}^{2}$ which contains $V_{1}^{2}$. The dimension of this parametrization is $2+1+1=4$. An ideal is formed by taking the ideal which is orthogonal to $Q$ in degree 2 and to the powers $V_{1}^{3}$ and $V_{1}^{4}$ in degrees 3 and 4 respectively. After a change of variables, we can assume that the flag is orthogonal to $\langle x\rangle \subset\langle x, y\rangle \subset S_{2}$. Then the degree 2 generators of $I$ are $x^{2}, x y, x z$ and another quadric. It is easy to see that these only generate a codimension 2 subspace of $S_{3}$. Conversely, for any ideal with this property, the orthogonal cubic has a 1dimensional space of derivatives. Furthermore, there exists a homogeneous ideal with Hilbert function $(1,3,2,2,1)$ contained in the original ideal. The cubics orthogonal to these have a 2-dimensional space of derivatives, so we can write them in terms of a 2-dimensional space of the dual variables. These two vector spaces determine the flag, and the parametrization is bijective on closed points. In particular, $\mathscr{E}$ is irreducible of dimension 4.

Lemma 4.7. The preimage $Z:=\pi^{-1}(\mathscr{L})$ is irreducible of dimension 11 .

Proof. By Lemma 4.6, it suffices to prove that the fibers of $\pi$ over $\mathscr{L}$ are irreducible and 7-dimensional. Let $I$ be a point in $\mathscr{L}$. As in the proof of Lemma 4.6, we can assume that the ideal corresponding to a point of $\mathscr{E}$ is generated by $x^{2}, x y, x z, q, c$, where $q$ and $c$ are a homogeneous quadric and cubic respectively. A point $J$ in the fiber must be generated by $\mathfrak{m}^{5}$ and

$$
\begin{array}{lll}
g_{1}:=x^{2}+a_{1} z^{3}+b_{1} z^{4}, & g_{2}:=x y+a_{2} z^{3}+b_{2} z^{4}, & \\
g_{3}:=x z+a_{3} z^{3}+b_{3} z^{4}, & g_{4}:=q+a_{4} z^{3}+b_{4} z^{4}, & g_{5}:=c+b_{5} z^{4} .
\end{array}
$$

The $a_{i}, b_{i}$ are not necessarily free. We must impose additional conditions to ensure the initial ideal for the weight vector $(-1,-1,-1)$ is no larger than $I$. In particular, we must have

$$
\begin{aligned}
& z g_{1}-x g_{3}=a_{1} z^{4}+b_{1} z^{5}-a_{3} x z^{3}-b_{3} x z^{4} \in J, \\
& z g_{2}-y g_{3}=a_{2} z^{4}+b_{2} z^{5}-a_{3} y z^{3}-b_{3} y z^{4} \in J .
\end{aligned}
$$


This implies $a_{1}=a_{2}=0$, because the final three terms of each expression are already in $J$. By Buchberger's criterion, it is also sufficient for these conditions to be satisfied. Therefore the fibers are 7-dimensional.

Lemma 4.8. The preimage $U:=\pi^{-1}(\mathcal{U})$ is irreducible of dimension 12.

Proof. By Proposition 3.8, it suffices to show that the fibers of $\pi$ over $U$ are irreducible of dimension 6. Let $I$ be an ideal in $U$. Let $V$ be the 1-dimensional subspace of $S_{1}^{*}$ such that $I_{3}$ is orthogonal to $\operatorname{Sym}_{3}(V)$ and let $q_{1}, \ldots, q_{4}$ be the degree 2 generators of $I$. Choose a basis $x, y, z$ of $S_{1}$ such that $x, y$ is a basis for $V^{\perp}$. Then any $J$ in $\phi^{-1}(I)$ is of the form

$$
\left\langle q_{i}+a_{i} z^{3}+b_{i} z^{4} \mid 1 \leq i \leq 4\right\rangle+\mathfrak{m}^{5} .
$$

We claim that forcing $\operatorname{in}_{(-1,-1,-1)}(J)=I$ imposes two linear conditions on the $a_{i}$ s. Using the table of isomorphism classes of $(1,3,2)$ algebras in [Poonen 2008], one can check that for any 4-dimensional subspace $\left\langle q_{1}, q_{2}, q_{3}, q_{4}\right\rangle$ of $\operatorname{Sym}_{2}(V)$, the intersection of $\left\langle z q_{i} \mid 1 \leq i \leq 4\right\rangle_{3}$ and $\left\langle x q_{j}, y q_{j} \mid 1 \leq j \leq 4\right\rangle_{3}$ is 2-dimensional. After choosing a different basis for $Q$, we may assume $z q_{1}, z q_{2} \in\left\langle x q_{j}, y q_{j} \mid 1 \leq j \leq 4\right\rangle$. By using a similar argument to the one in Lemma 4.7, we see $a_{1}=a_{2}=0$. Since the only other linear syzygies among the $q_{i}^{\prime} s$ have no $z$ coefficients and $x z^{3}, y z^{3}$ and $\mathfrak{m}^{5}$ are in the ideal, these are the only conditions imposed. Therefore, the fiber is 6-dimensional.

Therefore, it suffices to show the following irreducible sets are contained in the smoothable component.

$$
H_{(1, d, 1, \ldots, 1)}^{d}, H_{(1, d, 2)}^{d}, P_{d}, Q_{d}, H_{(1, d, 2,2)}^{d}, H_{(1,3,4)}^{3}, H_{(1,3,3)}^{3}, H_{(1,3,3,1)}^{3}, U, Z .
$$

Smoothable algebras. In this section we prove that the irreducible sets

$$
H_{(1, d, 1, \ldots, 1)}^{d}, H_{(1, d, 2)}^{d}, P_{d}, Q_{d}, H_{(1, d, 2,2)}^{d}, H_{(1,3,4)}^{3},
$$

are in the smoothable component by showing that a generic algebra in each is smoothable. We then show that the remaining irreducible sets,

$$
H_{(1,3,3)}^{3}, H_{(1,3,3,1)}^{3}, U, Z \text {, }
$$

are in the smoothable component by finding a point in each which is smoothable and a smooth point on the Hilbert scheme.

Proposition 4.9. All algebras in $H_{(1, d, 1, \ldots, 1)}^{d}$ are smoothable.

Proof. We prove this by induction on $d$. Note the $d=1$ case is trivial. Let $m$ be the greatest integer such that $h_{m}$ is nonzero. Then, by Proposition 4.4 we can take a generic ideal to be

$$
I=\left\langle x_{1}^{2}-x_{d}^{m}, \ldots, x_{d-1}^{2}-x_{d}^{m}, x_{d}^{m+1}\right\rangle+\left\langle x_{i} x_{j} \mid i \neq j\right\rangle .
$$


We define $J$ to be

$$
J=\left\langle x_{1}^{2}+x_{1}-x_{d}^{m}, \ldots, x_{d-1}^{2}-x_{d}^{m}, x_{d}^{m+1}\right\rangle+\left\langle x_{i} x_{j} \mid i \neq j\right\rangle .
$$

Note that $J$ admits a decomposition as $J=J_{1} \cap J_{2}$ where $J_{1}=\left\langle x_{1}+1, x_{2}, x_{3}, \ldots x_{d}\right\rangle$ and

$$
J_{2}=\left\langle x_{1}-x_{d}^{m}, x_{2}^{2}-x_{d}^{m}, \ldots, x_{d-1}^{2}-x_{d}^{m}, x_{d}^{m+1}\right\rangle+\left\langle x_{i} x_{j} \mid i \neq j\right\rangle .
$$

As the Hilbert function of $J_{2}$ equals $(1, d-1,1, \ldots, 1)$, the inductive hypothesis implies that $J_{2}$ is smoothable. Thus $J$ itself is also smoothable. Next note that $I \subset \operatorname{in}_{(m, \ldots, m, 2)}(J)$. Since both $I$ and $J$ have the same colength, we obtain the equality $I=\operatorname{in}_{(m, \ldots, m, 2)}(J)$. The corresponding Gröbner degeneration induces a map $\mathbb{A}^{1} \rightarrow R_{n}^{d}$ which sends 0 to $I$. Thus $I$ is smoothable.

Proposition 4.10. All algebras in $H_{(1, d, 2)}^{d}$ are smoothable.

Proof. The proof is by induction on $d$. The case $d=2$ follows from Theorem 2.4 of [Fogarty 1968].

Assume $d$ is at least 3. Note that $I_{2}^{\perp}$ defines a pencils of quadrics in $d$-variables. It then follows from [Harris 1992, Lemma 22.42] that, up to isomorphism, a generic ideal in $H_{(1, d, 2)}^{d}$ is of the form

$$
I=\left\langle x_{i} x_{j} \mid i \neq j\right\rangle+\left\langle x_{i}^{2}-a_{i} x_{d-1}^{2}-b_{i} x_{d}^{2} \mid 1 \leq i \leq d-2\right\rangle,
$$

with $a_{i}$ and $b_{i}$ elements of $k$.

Define

$$
\begin{aligned}
& J_{1}=\left\langle x_{i} x_{j} \mid i \neq j\right\rangle+\left\langle x_{1}+a_{1} x_{n-1}^{2}+b_{1} x_{d}^{2}, x_{i}^{2}-a_{i} x_{d-1}^{2}-b_{i} x_{d}^{2} \mid 2 \leq i \leq d-2\right\rangle, \\
& J_{2}=\left\langle x_{1}-1, x_{2}, \ldots, x_{d}\right\rangle .
\end{aligned}
$$

Since $J_{1}$ has Hilbert function $(1, d-1,2)$, it is thus smoothable by the induction hypothesis. One can check that $I=\operatorname{in}_{(1, \ldots, 1)}\left(J_{1} \cap J_{2}\right)$. Therefore $I$ is smoothable.

Proposition 4.11. All algebras in $P_{d}$ are smoothable.

Proof. Let $I$ be a generic ideal in $P_{d}$. After a change of variables we may assume

$$
I=\left\langle x_{i} x_{j}, x_{\ell}^{2}+x_{1}^{3}, x_{1}^{3}-x_{2}^{3} \mid 1 \leq i<j \leq d, 2<\ell \leq d\right\rangle .
$$

One can check

$$
\begin{aligned}
I=\operatorname{in}_{(2,2,3, \ldots, 3)}\left(\left\langle x_{1}+1, x_{j} \mid j>1\right\rangle\right. & \\
& \left.\left\langle x_{i} x_{j}, x_{\ell}^{2}+x_{1}^{2}, x_{1}^{2}-x_{2}^{3} \mid 1 \leq i<j \leq d, 2<\ell \leq d\right\rangle\right) .
\end{aligned}
$$

The second ideal in the intersection has Hilbert function $(1, d, 1,1)$, hence is smoothable by Proposition 4.9. It follows that $I$ is smoothable. 
Proposition 4.12. All algebras in $Q_{d}$ are smoothable.

Proof. Let $I$ be a generic ideal in $Q_{d}$. After a change of variables, we may assume

$$
I=\left\langle x_{1} x_{\ell}, x_{i} x_{j}+b_{(i, j)} x_{1}^{3}, x_{k}^{2}-x_{k+1}^{2}+b_{k} x_{1}^{3} \mid \ell \neq 1,1<i<j \leq d, 1<k<d\right\rangle .
$$

Define

$$
\begin{aligned}
& J_{1}=\left\langle x_{1} x_{\ell}, x_{i} x_{j}+b_{(i, j)} x_{1}^{2}, x_{k}^{2}-x_{k+1}^{2}+b_{k} x_{1}^{2} \mid \ell \neq 1,1<i<j \leq d, 1<k<d\right\rangle, \\
& J_{2}=\left\langle x_{1}+1, x_{2}, \ldots, x_{d}\right\rangle .
\end{aligned}
$$

Then $J_{1}$ has Hilbert function $(1, d, 2)$ so is smoothable by Proposition 4.10. One can check that $I=\operatorname{in}_{(2,3, \ldots, 3)}\left(J_{1} \cap J_{2}\right)$, and thus $I$ is smoothable.

Proposition 4.13. All algebras in $H_{(1, d, 2,2)}^{d}$ are smoothable.

Proof. Let $I$ be a generic ideal with Hilbert function $(1, d, 2,2)$. After a change of variable, we may assume $(\pi(I))_{2}^{\perp}=\left\langle y_{1}^{2}, y_{2}^{2}\right\rangle$. Thus $I$ must be of the form

$$
\left\langle x_{\ell}^{2}-a_{\ell \ell} x_{1}^{3}-b_{\ell \ell} x_{2}^{3}, x_{i} x_{j}-a_{i j} x_{1}^{3}-b_{i j} x_{2}^{3} \mid i<j, 2<\ell\right\rangle+\mathfrak{m}^{4} .
$$

Note $I$ determines a symmetric bilinear map

$$
\begin{aligned}
\phi:\left(\mathfrak{m}: \mathfrak{m}^{3}\right) / \mathfrak{m}^{2} \times\left(\mathfrak{m}: \mathfrak{m}^{3}\right) / \mathfrak{m}^{2} & \rightarrow \mathfrak{m}^{3} \cong k^{2}, \\
\left(x_{i}, x_{j}\right) & \mapsto a_{i j} x_{1}^{3}+b_{i j} x_{2}^{3} .
\end{aligned}
$$

By composing $\phi$ with projections onto the two coordinates, we get a pair of symmetric bilinear forms. For a generic $\phi$, these are linearly independent and their span is invariant under a change of basis on $\mathrm{m}^{3}$. By [Harris 1992, Lemma 22.42], there exists a basis for $\left(\mathfrak{m}: \mathfrak{m}^{3}\right) / \mathfrak{m}^{2}$ and $\mathfrak{m}^{3}$ such that these bilinear forms are represented by diagonal matrices. Thus $I$ has the following form

$$
\left\langle x_{\ell}^{2}-a_{\ell} x_{1}^{3}-b_{\ell} x_{2}^{3}, x_{i} x_{j}-a_{i j} x_{1}^{3}-b_{i j} x_{2}^{3} \mid i<j, 2<\ell\right\rangle+\mathfrak{m}^{4},
$$

where $a_{i j}=b_{i j}=0$ if $i$ and $j$ are both greater than 2 and $a_{\ell}, b_{\ell}$ are nonzero for all $\ell>2$. After suitable changes of variable, we may assume $b_{i j}=a_{i j}=0$ for all $i, j$. This gives the ideal

$$
I=\left\langle x_{\ell}^{2}-a_{\ell} x_{1}^{3}-b_{\ell} x_{2}^{3}, x_{i} x_{j}, x_{1}^{4}, x_{2}^{4} \mid i<j, 2<\ell\right\rangle .
$$

Now consider the following ideals:

$$
\begin{aligned}
& J_{1}:=\left\langle x_{\ell}^{2}-a_{\ell} x_{1}^{2}-b_{\ell} x_{2}^{3}, x_{i} x_{j}, x_{1}^{3}, x_{2}^{4} \mid i<j, 2<\ell\right\rangle, \\
& J_{2}:=\left\langle x_{1}+1, x_{2}, \ldots, x_{d}\right\rangle .
\end{aligned}
$$

Note $J_{1}$ is a $(1, d, 2,1)$ ideal and in fact lies in the component $Q_{d}$, and therefore is smoothable by Proposition 4.12. One can check that $I=\operatorname{in}_{(2,2,3, \ldots, 3)}\left(J_{1} \cap J_{2}\right)$, and therefore $I$ is smoothable. 
Proposition 4.14. All algebras in $H_{(1,3,4)}^{3}$ are smoothable.

Proof. Such algebras are given by a 2-dimensional subspace of the space of quadratic forms, with isomorphisms given by the action of $\mathrm{GL}_{3}$. Arguing as in Proposition 4.10, we conclude that, up to isomorphism, a generic 2-dimensional space of quadrics is spanned by $x^{2}+z^{2}$ and $y^{2}+z^{2}$. Adding the necessary cubic generators, we conclude that $I=\left\langle y^{2}+z^{2}, x^{2}+z^{2}, z^{3}, y z^{2}, x z^{2}, x y z\right\rangle$ is a generic point of $H_{(1,3,4)}^{3}$.

Consider

$$
J=\left\langle y^{2}+z^{2}, x+x^{2}+z^{2}, z^{3}, y z^{2}, x z^{2}, x y z\right\rangle .
$$

Note that $J$ is the intersection of an ideal of colength 3 and an ideal of colength 5:

$$
J=\left\langle x+1, y^{2}, y z, z^{2}\right\rangle \cap\left\langle x+z^{2}, y^{2}+z^{2}, z^{3}, y z^{2}\right\rangle .
$$

Since both ideals in the above intersection are smoothable, $J$ itself is smoothable. One can check that $I=\operatorname{in}_{(1,1,1)}(J)$. Therefore $I$ is smoothable.

To prove that the remaining families are smoothable, we show that they contain a smooth point which is also smoothable. For this, we use the following result, which is well known (see for example [Miller and Sturmfels 2005, Lemma 18.10] in characteristic 0 ), but we give the proof in arbitrary characteristic for the reader's convenience:

Proposition 4.15. All monomial ideals are smoothable.

Proof. Suppose we have a monomial ideal of colength $n$, written in multiindex notation $I=\left\langle\boldsymbol{x}^{\alpha^{(1)}}, \ldots, \boldsymbol{x}^{\alpha^{(m)}}\right\rangle$. Since $k$ is algebraically closed, we can pick an arbitrarily long sequence $a_{1}, a_{2}, \ldots$ consisting of distinct elements in $k$. Define

$$
f_{i}=\prod_{j=1}^{d}\left(\left(x_{j}-a_{1}\right)\left(x_{j}-a_{2}\right) \cdots\left(x_{j}-a_{\alpha_{j}^{(i)}}\right)\right) .
$$

Note that $\operatorname{in}\left(f_{i}\right)=\boldsymbol{x}^{\alpha^{(i)}}$ with respect to any global term order. Let $J$ be the ideal generated by the $f_{i}$ for $1 \leq i \leq m$ and then $\operatorname{in}(J) \supset I$ and so $J$ has colength at most $n$. However, for any standard monomial $\boldsymbol{x}^{\beta}$ in $I$, we have a distinct point $\left(a_{\beta_{1}}, \ldots, a_{\beta_{d}}\right)$ in $\mathbb{A}^{d}$, and each $f_{i}$ vanishes at this point. Therefore, $J$ must be the radical ideal vanishing at exactly these points and have initial ideal $I$. Thus, $I$ is smoothable.

Furthermore, the tangent space of an ideal $I$ in the Hilbert scheme is isomorphic to $\operatorname{Hom}_{S}(I, S / I)$. We use this fact to compute the dimension of the tangent space of a point $I$.

Proposition 4.16. All algebras in $H_{(1,3,3)}^{3}$ are smoothable. 
Proof. This irreducible set includes the smoothable monomial ideal $I$ generated by $x^{2}, y^{2}, z^{2}, x y z$. A direct computation shows $I$ has a 21-dimensional tangent space, so $I$ is a smooth point in $H_{7}^{3}$. Thus, any algebra in $H_{(1,3,3)}^{3}$ is smoothable.

Proposition 4.17. All algebras in $H_{(1,3,3,1)}^{3}$ are smoothable.

Proof. The ideal $I=\left\langle x^{2}, y^{2}, z^{2}\right\rangle$ in this locus is smoothable by Proposition 4.15, and one can check that the Hilbert scheme is smooth at this point as well. Therefore $H_{(1,3,3,1)}^{3}$ is contained in the smoothable component of the Hilbert scheme.

Proposition 4.18. All algebras in $Z$ are smoothable.

Proof. Consider $I=\left\langle x^{2}, x y, x z, y z, z^{3}-y^{4}\right\rangle \in Z$ and note that

$$
I=\operatorname{in}_{(1,0,0)}\left(\langle x+1, y, z\rangle \cap\left\langle x, y z, z^{3}-y^{4}\right\rangle\right) .
$$

The second ideal is smoothable [Fogarty 1968, Theorem 2.4], so $I$ is smoothable. One can also check $I$ is smooth in the Hilbert scheme by computing the dimension of $\operatorname{Hom}_{S}(I, S / I)$. Therefore $Z$ is contained in the smoothable component of the Hilbert scheme.

Proposition 4.19. All algebras in $U \subset H_{(1,3,2,1,1)}^{3}$ are smoothable.

Proof. Consider the ideal $I=\left\langle x^{2}, x y-z^{4}, y^{2}-x z, y z\right\rangle \in U$. One can check that

$$
I=\operatorname{in}_{(7,5,3)}\left(\langle x, y, z-1\rangle \cap\left\langle x^{2}, x y-z^{3}, y^{2}-x z, y z\right\rangle\right) .
$$

The second ideal in the intersection is in $Q_{3}$ and therefore smoothable by Proposition 4.12. Therefore $I$ is smoothable by the same argument in the proof of Proposition 4.14. One can also check $I$ has a 24-dimensional tangent space in the Hilbert scheme and is thus smooth. Therefore $U$ is contained in the smoothable component.

Theorem 4.20. With the exception of local algebras with Hilbert function $(1,4,3)$, every algebra with $n \leq 8$ is smoothable.

Proof. The possible Hilbert functions are exactly the Hilbert functions of monomial ideals, and for $d$ at least 3, one can check that there are no possibilities other those listed in Table 4.1. For $d$ at most 2, smoothability follows from Theorem 2.4 of [Fogarty 1968]

In particular, this implies that there are no components other than the ones listed in Theorem 1.1. 


\begin{tabular}{|c|c|c|c|c|c|}
\hline degree & $\begin{array}{l}\text { Hilbert } \\
\text { function } \boldsymbol{h}\end{array}$ & $\mathcal{H}_{\boldsymbol{h}}^{d}$ c.d. & reference & $H_{h}^{d}$ c.d. & $\begin{array}{l}\text { smoothability } \\
\text { reference }\end{array}$ \\
\hline 4 & 1,3 & 0 & Prop. 3.3 & 0 & Prop. 4.15 \\
\hline \multirow[t]{2}{*}{5} & $1,3,1$ & 5 & Prop. 3.3 & 5 & Prop. 4.9 \\
\hline & 1,4 & 0 & Prop. 3.3 & 0 & Prop. 4.15 \\
\hline \multirow[t]{3}{*}{6} & $1,3,1,1$ & 2 & Prop. 3.4 & 7 & Prop. 4.10 \\
\hline & $1,4,1$ & 9 & Prop. 3.3 & 9 & Prop. 4.9 \\
\hline & 1,5 & 0 & Prop. 3.3 & 0 & Prop. 4.15 \\
\hline \multirow[t]{7}{*}{7} & $1,3,1,1,1$ & 2 & Prop. 3.4 & 9 & Prop. 4.9 \\
\hline & $1,3,2,1$ & 5,6 & Thm. 3.5 & 9,10 & Prop. 4.12, 4.11 \\
\hline & $1,3,3$ & 9 & Prop. 3.3 & 9 & Prop. 4.16 \\
\hline & $1,4,1,1$ & 3 & Prop. 3.4 & 12 & Prop. 4.9 \\
\hline & $1,4,2$ & 16 & Prop. 3.3 & 16 & Prop. 4.10 \\
\hline & $1,5,1$ & 14 & Prop. 3.3 & 14 & Prop. 4.9 \\
\hline & 1,6 & 0 & Prop. 3.3 & 0 & Prop. 4.15 \\
\hline \multirow[t]{12}{*}{8} & $1,3,1,1,1,1$ & 2 & Prop. 3.4 & 11 & Prop. 4.9 \\
\hline & $1,3,2,1,1$ & 6 & Prop. 3.8 & $11(?), 12$ & Prop. $4.19,4.18$ \\
\hline & $1,3,2,2$ & 4 & Prop. 3.6 & 12 & Prop. 4.13 \\
\hline & $1,3,3,1$ & 9 & Prop. 3.7 & 12 & Prop. 4.17 \\
\hline & $1,3,4$ & 8 & Prop. 3.3 & 8 & Prop. 4.14 \\
\hline & $1,4,1,1,1$ & 3 & Prop. 3.4 & 15 & Prop. 4.9 \\
\hline & $1,4,2,1$ & 7,11 & Thm. 3.5 & 15,19 & Prop. 4.12, 4.11 \\
\hline & $1,4,3$ & 21 & Prop. 3.3 & 21 & \\
\hline & $1,5,2$ & 26 & Prop. 3.3 & 26 & Prop. 4.10 \\
\hline & $1,5,1,1$ & 4 & Prop. 3.4 & 18 & Prop. 4.9 \\
\hline & $1,6,1$ & 20 & Prop. 3.3 & 20 & Prop. 4.9 \\
\hline & 1,7 & 0 & Prop. 3.3 & 0 & Prop. 4.15 \\
\hline
\end{tabular}

Table 4.1. Summary of the decomposition of Hilbert schemes by Hilbert function of the local algebra with $h_{1} \geq 3$. Here c.d. stands for "component dimensions". The component dimensions of $H_{\boldsymbol{h}}^{d}$ are computed using Propositions 4.3 and 4.4. In the case of $\boldsymbol{h}=$ $(1,3,2,1,1)$, Lemmas 4.7 and 4.8 show that $H_{h}^{d}$ is the union of two irreducible sets, but we don't know whether the smaller set is contained in the closure of the larger one.

\section{Characterization of smoothable points of $\mathrm{H}_{8}^{4}$}

In this section $k$ will denote a field of characteristic not 2 or 3, except for Section $5 \mathrm{D}$ where $k=\mathbb{C}$.

We show that besides the smoothable component, the Hilbert scheme $H_{8}^{4}$ contains a second component parametrizing the local algebras with $\boldsymbol{h}=(1,4,3)$. We 
prove that the intersection of the two components can be described as in Theorem 1.3, and as a result we determine exactly which algebras with Hilbert function $(1,4,3)$ are smoothable. We will use $G_{0}$ to denote the standard graded Hilbert scheme $\mathcal{H}_{(1,4,3)}^{4} \cong \operatorname{Gr}\left(7, S_{2}\right)$, which we will think of as a closed subscheme of $H_{8}^{4}$.

In Section 5B we introduce and investigate the Pfaffian which appears in Theorem 1.3, and we prove the crucial fact that it is the unique $\mathrm{GL}_{4}$-invariant of minimal degree. In Section 5C, we give a first approximation of the intersection locus. We then use the uniqueness results from Section 5B to prove Theorem 1.3. We begin by proving reducibility,

Proposition 5.1. For $d$ at least 4 , the Hilbert scheme $H_{8}^{d}$ is reducible.

Proof. It is sufficient to find a single ideal whose tangent space dimension is less than $8 d=\operatorname{dim} R_{8}^{d}$. Consider the ideal

$$
J=\left\langle x_{1}^{2}, x_{1} x_{2}, x_{2}^{2}, x_{3}^{2}, x_{3} x_{4}, x_{4}^{2}, x_{1} x_{4}+x_{2} x_{3}\right\rangle+\left\langle x_{i} \mid 4<i \leq d\right\rangle .
$$

The tangent space of $J$ in $H_{8}^{d}$ can be computed as $\operatorname{dim}_{k} \operatorname{Hom}_{S}(J, S / J)$. A direct computation shows that an arbitrary element of $\operatorname{Hom}_{S}(J, S / J)$ can be represented as a matrix

\begin{tabular}{|c|c|c|c|c|c|c|c|c|}
\hline & $x_{1}^{2}$ & $x_{1} x_{2}$ & $x_{2}^{2}$ & $x_{3}^{2}$ & $x_{3} x_{4}$ & $x_{4}^{2}$ & $x_{1} x_{4}+x_{2} x_{3}$ & $x_{i}$ \\
\hline 1 & 0 & 0 & 0 & 0 & 0 & 0 & 0 & $*$ \\
\hline$x_{1}$ & $2 a_{2}$ & $a_{1}$ & 0 & 0 & 0 & 0 & $a_{4}$ & $*$ \\
\hline$x_{2}$ & 0 & $a_{2}$ & $2 a_{1}$ & 0 & 0 & 0 & $a_{3}$ & $*$ \\
\hline$x_{3}$ & 0 & 0 & 0 & $2 a_{3}$ & $a_{4}$ & 0 & $a_{1}$ & $*$ \\
\hline$x_{4}$ & 0 & 0 & 0 & 0 & $a_{3}$ & $2 a_{4}$ & $a_{2}$ & $*$ \\
\hline$x_{1} x_{3}$ & $*$ & $*$ & $*$ & $*$ & $*$ & $*$ & $*$ & $*$ \\
\hline$x_{1} x_{4}$ & $*$ & $*$ & $*$ & $*$ & $*$ & $*$ & $*$ & $*$ \\
\hline$x_{2} x_{4}$ & $*$ & $*$ & $*$ & $*$ & $*$ & $*$ & $*$ & $*$ \\
\hline
\end{tabular}

where $i$ again ranges over $4<i \leq d$, the $a_{i}$ are any elements in $k$, and each $*$ represents an independent choice of an element of $k$. Thus, $\operatorname{dim}_{k} \operatorname{Hom}(J, S / J)=$ $4+21+8(d-4)=8 d-7$. The computation holds in all characteristics. Since $8 d-7<8 d=\operatorname{dim}\left(R_{8}^{d}\right)$, we conclude that $J$ is not smoothable and that $H_{8}^{d}$ is reducible.

Remark 5.2. This proposition holds with the same proof even when char $k=2,3$.

5A. The irreducible components of $\boldsymbol{H}_{\mathbf{8}}^{\mathbf{4}}$. Consider $H_{8}^{4}$ with its universal ideal sheaf $\mathscr{I}$ and let $\mathscr{A}=\mathscr{O}_{H_{8}^{4}}\left[x_{1}, \ldots, x_{4}\right] / \mathscr{I}$. On each open affine $U=\operatorname{Spec} B$ such that $\left.\mathscr{A}\right|_{U}$ is free, define $f_{i} \in B$ to be $\frac{1}{8} \operatorname{tr}\left(X_{i}\right)$ where $X_{i}$ is the operator on the free $B$-module $\mathscr{A}(U)$ defined by multiplication by $x_{i}$. We think of the $f_{i}$ as being the "center of mass" functions for the subscheme of $\mathbb{A}_{B}^{4}$ defined by $\left.\mathscr{I}\right|_{U}$. Note that the 
definitions of $f_{i}$ commute with localization and thus they lift to define elements $f_{i} \in \Gamma\left(H_{8}^{4}, O_{H_{8}^{4}}\right)$, which determine a morphism $f: H_{8}^{4} \rightarrow \mathbb{A}^{4}$.

Considered as an additive group, $\mathbb{A}^{4}$ acts on $H_{8}^{4}$ by translation. We define the "recentering" map $r$ to be the composition

$$
r: H_{8}^{4} \stackrel{-f \times \mathrm{id}}{\longrightarrow} \mathbb{A}^{4} \times H_{8}^{4} \longrightarrow H_{8}^{4} .
$$

By forgetting about the grading of ideals, we have a closed immersion $\imath$ of $G_{0} \cong \mathscr{H}_{(1,4,3)}^{4}$ into $H_{8}^{4}$ [Haiman and Sturmfels 2004, Proposition 1.5]. We define $G$ to be the preimage of this closed subscheme via the "recentering" map, that is, the fiber product

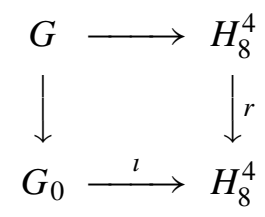

We define intersections $W:=G \cap R_{8}^{4}$ and $W_{0}:=G_{0} \cap R_{8}^{4}$. We will focus on $W_{0}$, and the following lemma shows that this is sufficient for describing $W$.

Lemma 5.3. We have isomorphisms $G \cong G_{0} \times \mathbb{A}^{4}$ and $W \cong W_{0} \times \mathbb{A}^{4}$.

Proof. We have a map $G \rightarrow G_{0}$, and a map $f: H_{8}^{4} \rightarrow \mathbb{A}^{4}$. We claim that the induced map $\phi: G \rightarrow \mathbb{A}^{4} \times G_{0}$ is an isomorphism.

Define $\psi: \mathbb{A}^{4} \times G_{0} \rightarrow H_{8}^{4}$ to be the closed immersion $\imath$ followed by translation. We work with an open affine $U \cong \operatorname{Spec} A \subset G_{0}$ such that the restriction $\left.\imath\right|_{U}$ corresponds to an ideal $I \subset A\left[x_{1}, \ldots, x_{4}\right]$ whose cokernel is a graded $A$-module with free components of ranks $(1,4,3)$. The map $\left.\psi\right|_{\mathbb{A}^{4} \times U}$ corresponds to the ideal $I^{\prime} \subset A\left[t_{1}, \ldots, t_{4}\right]\left[x_{1}^{\prime}, \ldots, x_{4}^{\prime}\right]$ where $I^{\prime}$ is the image of $I$ under the homomorphism of $A$-algebras that sends $x_{i}$ to $x_{i}^{\prime}+t_{i}$. Then $A\left[t_{1}, \ldots, t_{4}\right]\left[x_{1}^{\prime}, \ldots, x_{4}^{\prime}\right] / I^{\prime} \cong$ $\left(A\left[x_{1}, \ldots, x_{4}\right] / I\right)\left[t_{1}, \ldots, t_{4}\right]$ is a graded $A\left[t_{1}, \ldots, t_{4}\right]$-algebra with $x_{i}^{\prime}-t_{i}=x_{i}$ homogeneous of degree 1 . The key point is that as operators on a free $A\left[t_{1}, \ldots, t_{4}\right]$ module, the $x_{i}$ have trace zero, so the trace of the $x_{i}^{\prime}$ is $8 t_{i}$. Thus, $r \circ \psi: \mathbb{A}^{4} \times G_{0} \rightarrow$ $H_{8}^{4}$ corresponds to an ideal $I^{\prime \prime} \subset A\left[t_{1}, \ldots, t_{4}\right]\left[x_{1}^{\prime \prime}, \ldots, x_{4}^{\prime \prime}\right]$ which is the image of $I^{\prime}$ under the homomorphism that takes $x_{i}^{\prime}$ to $x_{i}^{\prime \prime}-t_{i}$. This is of course the extension of $I \subset A\left[x_{1}, \ldots, x_{4}\right]$ to $A\left[t_{1}, \ldots, t_{4}\right]\left[x_{1}^{\prime \prime}, \ldots, x_{4}^{\prime \prime}\right]$ with $x_{i}^{\prime \prime}=x_{i}$, and so $I^{\prime \prime}$ has the required properties such that $r \circ \psi$ factors through the closed immersion $l$. Thus, $\psi$ maps to $G$. Furthermore, we see that $r \circ \psi$ is the projection onto the first coordinate of $A^{4} \times G_{0}$ and $f \circ \psi$ is projection onto the second coordinate. Thus, $\phi \circ \psi$ is the identity.

Second, we check that the composition $\psi \circ \phi$ is the identity on $G$. This is clear because $\psi \circ \phi$ amounts to translation by $-f$ followed by translation by $f$.

The isomorphism for $W$ follows by the same argument. 
Lemma 5.4. $W$ and $W_{0}$ are prime divisors in $G$ and $G_{0}$ respectively.

Proof. The point $I=\left\langle x_{1}^{2}, x_{1} x_{2}, x_{2}^{2}, x_{3}^{2}, x_{3} x_{4}, x_{4}^{2}, x_{1} x_{4}\right\rangle$ belongs to $R_{8}^{4}$ and to $G$ and has a 33-dimensional tangent space in any characteristic. As a result, an open set $U$ around $I$ in the Hilbert scheme is a closed subscheme of a smooth 33-dimensional variety $Y$. By the subadditivity of codimension of intersections, as in [Harris 1992, Theorem 17.24], it follows that every component of $W$ through $I$ has codimension 1 in $G$.

To show integrality, fix a monomial ideal $M_{\lambda} \in G_{0}$, and let $U_{\lambda} \subset H_{8}^{4}$ be the corresponding open set, as in our discussion of coordinates for the Hilbert scheme of points (page 766). For any $I \in U_{\lambda}$ the initial ideal $\operatorname{in}_{(1,1,1,1)}(I) \in G_{0} \cap U_{\lambda}$ and is generated by the $(1,1,1,1)$-leading forms of the given generating set of $I$ and all cubics. Thus we may define a projection morphism $\pi: U_{\lambda} \rightarrow G_{0} \cap U_{\lambda}$ which corresponds to taking the $(1,1,1,1)$-initial ideal. Since $R_{8}^{4}$ is integral, so is the image $\pi\left(R_{8}^{4} \cap U_{\lambda}\right)=W_{0} \cap U_{\lambda}$. Thus $W_{0}$ and $W_{0} \times \mathbb{A}^{4} \cong W$ are integral.

5B. A GL $\mathbf{G L}_{4}$-invariant of a system of three quadrics. In this section we study the Pfaffian which appears in the statement of Theorem 1.3. Recall that any $I \in G_{0}$ defines a 3-dimensional subspace $I_{2}^{\perp} \subset S_{2}^{*}$.

Let $Q_{1}, Q_{2}, Q_{3}$ be a basis of quadrics for $I_{2}^{\perp}$, let $A_{1}, A_{2}, A_{3}$ be the symmetric $4 \times 4$ matrices which represent the $Q_{i}$ via $\vec{y}^{t} A_{i} \vec{y}=Q_{i}$ where $\vec{y}$ is the vector of formal variables $\left(y_{1}, y_{2}, y_{3}, y_{4}\right)$.

Definition 5.5. The Salmon-Turnbull Pfaffian is the Pfaffian (that is, the square root of the determinant) of the skew-symmetric $12 \times 12$ matrix

$$
M_{I}=\left(\begin{array}{rrr}
0 & A_{1} & -A_{2} \\
-A_{1} & 0 & A_{3} \\
A_{2} & -A_{3} & 0
\end{array}\right) .
$$

Lemma 5.6. The Salmon-Turnbull Pfaffian of $M_{I}$ coincides up to scaling with the Pfaffian of the skew-symmetric bilinear form $\langle,\rangle_{I}:\left(S_{1} \otimes S_{2} / I_{2}\right)^{\otimes 2} \rightarrow \bigwedge^{3} S_{2} / I_{2} \cong k$ defined by

$$
\left\langle l_{1} \otimes q_{1}, l_{2} \otimes q_{2}\right\rangle_{I}=\left(l_{1} l_{2}\right) \wedge q_{1} \wedge q_{2} .
$$

In particular, the vanishing of the Salmon-Turnbull Pfaffian is independent of the choice of basis of $I_{2}^{\perp}$ and invariant under the $\mathrm{GL}_{4}$ action induced by linear change of coordinates on $S$.

Proof. Let $m_{1}, m_{2}, m_{3}$ be any basis of $S_{2} / I_{2}$ and let $x_{1}, x_{2}, x_{3}, x_{4}$ be a basis for $S_{1}$. Let $A_{i}$ be the matrix representation with respect to this basis of the symmetric bilinear form obtained by composing multiplication $S_{1} \otimes S_{1} \rightarrow S_{2} / I_{2}$ with projection onto $m_{i}$. Note that if $m_{1}, m_{2}, m_{3}$ form a basis dual to $\frac{1}{2} Q_{1}, \frac{1}{2} Q_{2}, \frac{1}{2} Q_{3}$ then this definition of $A_{i}$ agrees with the definition of $A_{i}$ above. Thus, $x_{j} x_{j^{\prime}}=$ 
$\sum_{i}\left(A_{i}\right)_{j j^{\prime}} m_{i}$ where $\left(A_{i}\right)_{j j^{\prime}}$ is the $\left(j, j^{\prime}\right)$ entry of $A_{i}$. Then we will use the basis $x_{1} \otimes m_{3}, x_{2} \otimes m_{3}, \ldots, x_{4} \otimes m_{1}$ for $S_{1} \otimes S_{2} / I_{2}$. We compute the matrix representation of $\langle,\rangle_{I}$ in this basis:

$$
\begin{aligned}
\left\langle x_{j} \otimes m_{i}, x_{j^{\prime}} \otimes m_{i^{\prime}}\right\rangle_{I} & =\left(x_{j} x_{j^{\prime}}\right) \wedge m_{i} \wedge m_{i^{\prime}} \\
& =\left(\sum_{1 \leq \ell \leq 3}\left(A_{\ell}\right)_{j j^{\prime}} m_{\ell}\right) \wedge m_{i} \wedge m_{i}^{\prime}
\end{aligned}
$$

If $i=i^{\prime}$, this quantity will be zero. Otherwise, let $i^{\prime \prime}$ be the index which is not $i$ or $i^{\prime}$ and then we get

$$
=\left(A_{i^{\prime \prime}}\right)_{j j^{\prime}} m_{i^{\prime \prime}} \wedge m_{i} \wedge m_{i^{\prime}}= \pm\left(A_{i^{\prime \prime}}\right)_{j j^{\prime}} m_{1} \wedge m_{2} \wedge m_{3},
$$

where \pm is the sign of the permutation which sends $1,2,3$ to $i^{\prime \prime}, i, i^{\prime}$ respectively. Thus, with $m_{1} \wedge m_{2} \wedge m_{3}$ as the basis for $\wedge^{3} S_{2} / I_{2},\langle,\rangle_{I}$ is represented as

$$
\left(\begin{array}{ccc}
0 & A_{1} & -A_{2} \\
-A_{1} & 0 & A_{3} \\
A_{2} & -A_{3} & 0
\end{array}\right) \text {. }
$$

Since the vanishing of the Salmon-Turnbull Pfaffian depends only on the vector subspace $I_{2}^{\perp} \subset S_{2}^{*}$, it defines a function $P$ on $G_{0}$ which is homogeneous of degree 2 in the Plücker coordinates. We next show that the Salmon-Turnbull Pfaffian is irreducible and that, over the complex numbers, it is uniquely determined by its degree and $\mathrm{GL}_{4}$-invariance.

Lemma 5.7. There are no polynomials of degree 1 in the Plücker coordinates of $G_{0}$ whose vanishing locus is invariant under the action of the algebraic group $\mathrm{GL}_{4}$. Therefore, the Salmon-Turnbull Pfaffian is irreducible.

Proof. We may prove this lemma by passing to the algebraic closure, and we thus assume that $k$ is algebraically closed. Let $W=\bigwedge^{3} S_{2}^{*}$ and consider the Plücker embedding of $\operatorname{Gr}\left(3, S_{2}^{*}\right)$ in $\mathbb{P}(W)=\operatorname{Proj}(R)$ where $R$ is the polynomial ring $k\left[p_{i j \ell}\right]$ where $\{i, j, \ell\}$ runs over all unordered triplets of monomials in $S_{2}^{*}$. The Plücker coordinate ring $A$ is the quotient of $R$ by a homogeneous ideal $J$. In each degree $e$, we obtain a split exact sequence of $\mathrm{GL}_{4}$-representations:

$$
0 \rightarrow J_{e} \rightarrow \operatorname{Sym}_{e}(W) \rightarrow A_{e} \rightarrow 0 .
$$

Since $J_{1}=0$ we have $\operatorname{Sym}_{1}(W)=A_{1}$, and it suffices to show that this has no 1-dimensional subrepresentations. Given a monomial $i \in S_{2}^{*}$ let $\alpha_{i} \in \mathbb{N}^{4}$ be its multiindex. For $\theta=\left(\theta_{1}, \ldots, \theta_{4}\right)$, let $L$ be the diagonal matrix with $L_{m m}=\theta_{m}$. The action of $L$ on the Plücker coordinate $p_{i j \ell}$ is to scale it by $\theta^{\alpha_{i}+\alpha_{j}+\alpha_{\ell}}$. 
Suppose that there exists an invariant polynomial $F=\sum c_{i j \ell} p_{i j \ell}$ in $\operatorname{Sym}_{1}(W)$. Then $L \cdot F=\lambda F$ for some $\lambda \in k^{\times}$. But since $L \cdot F=\sum c_{i j \ell} \theta^{\alpha_{i}+\alpha_{j}+\alpha_{\ell}} p_{i j \ell}$ it follows that whenever $c_{i j \ell}$ and $c_{i^{\prime} j^{\prime} \ell^{\prime}}$ are both nonzero, then $\alpha_{i}+\alpha_{j}+\alpha_{\ell}=\alpha_{i^{\prime}}+$ $\alpha_{j^{\prime}}+\alpha_{\ell^{\prime}}$. However there are no multiindices of total degree 6 which are also symmetric in $\theta_{1}, \theta_{2}, \theta_{3}, \theta_{4}$. Thus each $c_{i j \ell}=0$ and there are no nontrivial $\mathrm{GL}_{4}$ invariant polynomials of degree 1 . In particular no product of linear polynomials is $\mathrm{GL}_{4}$-invariant, and thus the Salmon-Turnbull Pfaffian is irreducible.

Lemma 5.8. If $k=\mathbb{C}$, then there is a polynomial of degree 2 in the Plücker coordinates, unique up to scaling, whose vanishing locus is $\mathrm{GL}_{4}$-invariant. This is the Salmon-Turnbull Pfaffian.

Proof. We take the same notation as in the proof of Lemma 5.7 and recall that we have a split exact sequence of $\mathrm{GL}_{4}(\mathbb{C})$-representations:

$$
0 \rightarrow J_{2} \rightarrow \operatorname{Sym}_{2}(W) \rightarrow A_{2} \rightarrow 0 .
$$

We determine the irreducible subrepresentations of $\operatorname{Sym}_{2}(W)$ by computing the following Schur function decomposition of its character $\chi$ :

$$
\begin{aligned}
& \chi=s_{(8,2,2)}+s_{(7,4,1)}+2 s_{(7,3,1,1)}+s_{(7,2,1,1)}+s_{(6,6)}+3 s_{(6,4,2)}+s_{(6,4,1,1)} \\
&+2 s_{(6,4,1,1)}+2 s_{(6,3,2,1)}+s_{(6,2,2,2)}+ 2 s_{(5,5,1,1)}+s_{(5,4,3)}+s_{(5,4,2,1)}+s_{(5,3,3,1)} \\
&+s_{(4,4,4)}+s_{(4,4,3,1)}+2 s_{(4,4,2,2)}+s_{(3,3,3,3)} .
\end{aligned}
$$

We conclude that $\operatorname{Sym}_{2}(W)$ contains a unique 1-dimensional subrepresentation with character $s_{(3,3,3,3)}$. It follows from this and Lemma 5.6 that, over $\mathbb{C}$, the Salmon-Turnbull Pfaffian is the only $\mathrm{GL}_{4}$-invariant of degree 2 in the Plücker coordinates.

Remark 5.9. Salmon gives a geometric description of the Salmon-Turnbull Pfaffian [Salmon 1874, pp. 242-244], where he shows that the Pfaffian vanishes whenever there exists a cubic form $C$ and three linear differential operators $d_{1}, d_{2}, d_{3}$ such that $d_{i} C=Q_{i}$. Turnbull also describes this invariant in his study of ternary quadrics [Turnbull 1922].

5C. A first approximation to the intersection locus. Any point $I$ in $W_{0}$ is a singular point in the Hilbert scheme. In Lemma 5.11, we construct an equation that cuts out the singular locus over an open set of $G_{0}$. The local equation defines a nonreduced divisor whose support contains $W_{0}$. The following subsets of $G_{0}$ will be used in this section:

$$
\begin{aligned}
& G_{0}^{\prime}:=\left\{I \in G_{0} \mid \text { the ideal } I \text { is generated in degree } 2\right\}, \\
& Z_{1}:=G_{0} \backslash G_{0}^{\prime}, \\
& Z_{2}:=\left\{I \in G_{0} \mid \operatorname{Hom}(I, S / I)_{-2} \neq 0\right\} .
\end{aligned}
$$


Note that $G_{0}^{\prime}$ is open in $G_{0}$ and that every ideal in $G_{0}^{\prime}$ is generated by seven quadrics. The set $Z_{2}$ will be used in Lemma 5.16. If $I$ is any ideal in $G_{0}$, the tangent space $\operatorname{Hom}_{S}(I, S / I)$ is graded. The following lemma shows that if we want to determine whether $I$ is a singular point in the Hilbert scheme, then it suffices to compute only the degree -1 component of the tangent space.

Lemma 5.10. For any $I \in G_{0}^{\prime}$ we have $\operatorname{dim}_{k} \operatorname{Hom}_{S}(I, S / I)_{-1} \geq 4$, and $I$ is singular in $H_{8}^{4}$ if and only if $\operatorname{dim}_{k} \operatorname{Hom}_{S}(I, S / I)_{-1} \geq 5$.

Proof. Since $S / I$ is concentrated in degrees 0,1 and 2 , and $I \in G_{0}^{\prime}$ is minimally generated only in degree 2 , we have that $\operatorname{Hom}_{S}(I, S / I)$ is concentrated in degrees $0,-1,-2$. Furthermore, since $I \in G_{0}^{\prime}$ we have that $\operatorname{dim}_{k} \operatorname{Hom}_{S}(I, S / I)_{0}=21$, because any $k$-linear map $I_{2} \rightarrow(S / I)_{2}$ will be $S$-linear. Next, note that the morphisms $t_{i}: I_{2} \rightarrow(S / I)_{1}$ mapping $q_{j}$ to the class of $\partial q_{j} / \partial x_{i}$ are $S$-linear morphisms, and thus we have $\operatorname{Hom}_{S}(I, S / I)_{-1}$ is at least 4-dimensional.

Since the dimension of $G_{0}^{\prime}$ is $25, I$ must be singular if $\operatorname{dim}_{k} \operatorname{Hom}_{S}(I, S / I)_{-1}>4$. Conversely, assume for contradiction that there exists an $I$ such that $I$ is singular and dimension of $\operatorname{Hom}_{S}(I, S / I)_{-1}$ is exactly 4. Since $I$ is singular, we have that $\operatorname{Hom}_{S}(I, S / I)_{-2}$ is nontrivial. Let $\phi \in \operatorname{Hom}_{S}(I, S / I)_{-2}$ be a nonzero map. By changing the generators of $I$ we may assume that $\phi\left(q_{i}\right)=0$ for $i=1, \ldots, 6$ and $\phi\left(q_{7}\right)=1$. Now the vector space $\left\langle x_{1} \phi, x_{2} \phi, x_{3} \phi, x_{4} \phi\right\rangle$ is a 4-dimensional subspace of $\operatorname{Hom}(I, S / I)_{-1}$. Since we have assumed that $\operatorname{dim}_{k} \operatorname{Hom}(I, S / I)_{-1}=4$ it must be the case that the space $\left\langle x_{1} \phi, x_{2} \phi, x_{3} \phi, x_{4} \phi\right\rangle$ equals the space $\left\langle t_{1}, \ldots, t_{4}\right\rangle$. However, this would imply that all partial derivatives of $q_{1}$ are zero, which is impossible.

Now we will investigate those ideals which have extra tangent vectors in degree -1 . If $\phi: I_{2} \rightarrow(S / I)_{1}$ is a $k$-linear map then $\phi$ will be $S$-linear if and only if $\phi$ satisfies the syzygies of $I$ modulo $I$. In other words, $\phi$ should belong to the kernel of

$$
\begin{aligned}
\operatorname{Hom}_{k}\left(I_{2},(S / I)_{1}\right) & \rightarrow \operatorname{Hom}_{k}(\operatorname{Syz}(I),(S / I)), \\
\phi & \mapsto(\sigma \mapsto \overline{\sigma(\phi)}) .
\end{aligned}
$$

Since $I$ contains $\mathfrak{m}^{3}$ and is generated by quadrics, it suffices to consider linear syzygies $\sigma$ and we have an exact sequence

$$
0 \rightarrow \operatorname{Hom}_{S}(I, S / I)_{-1} \longrightarrow \operatorname{Hom}_{k}\left(I_{2},(S / I)_{1}\right) \stackrel{\psi}{\longrightarrow} \operatorname{Hom}_{k}\left(\operatorname{Syz}(I)_{1},(S / I)_{2}\right),
$$

where $\operatorname{Syz}(I)_{1}$ is the vector space of linear syzygies. We see that the $t_{i}$ from the previous lemma span a 4-dimensional subspace $T$ of the kernel of $\psi$. We obtain

$$
\operatorname{Hom}_{k}(I, S / I)_{-1} / T \stackrel{\bar{\psi}}{\longrightarrow} \operatorname{Hom}_{k}\left(\operatorname{Syz}(I)_{1},(S / I)_{2}\right),
$$


and $I \in G_{0}^{\prime}$ will be a singular point if and only if $\operatorname{ker}(\bar{\psi}) \neq 0$. Since $I$ is generated by quadrics, it follows that $\operatorname{Syz}(I)_{1}$ has dimension $4 \cdot 7-20=8$. Therefore $\bar{\psi}$ is a map between 24-dimensional spaces. Thus $\operatorname{det}(\bar{\psi})$ vanishes if and only if $I \in G_{0}^{\prime}$ is a singular point in $H_{8}^{4}$.

The global version of this determinant will give a divisor whose support contains $W_{0}$. On $G_{0}^{\prime}$ we have the $\mathcal{O}_{G_{0}^{\prime}}$-algebra $\mathscr{S}:=\mathscr{O}_{G_{0}^{\prime}}\left[x_{1}, x_{2}, x_{3}, x_{4}\right]$, which is graded in the standard way, $\mathscr{Y}=\oplus_{i} \mathscr{I}_{i}$. We have a graded universal ideal sheaf $\mathscr{I}=\oplus \mathscr{I}_{i}$, and a universal sheaf of graded algebras $\mathscr{S} / \mathscr{I}=\oplus_{i} \mathscr{S}_{i} / \mathscr{I}_{i}$. For all $i$ the sheaves $\mathscr{S}_{i}, \mathscr{I}_{i}$ and $\mathscr{S}_{i} / \mathscr{I}_{i}$ are coherent locally free $O_{G_{0}^{\prime}}$-modules.

Let $\mu: \Phi_{2} \otimes \mathscr{S}_{1} \rightarrow \mathscr{I}_{3}$ be the multiplication map. Surjectivity of this map follows from the definition of $G_{0}^{\prime}$. We define $\mathscr{K}_{1}$ to be the kernel of this map, so that we have the exact sequence

$$
0 \rightarrow \mathscr{K}_{1} \rightarrow \mathscr{I}_{2} \otimes \mathscr{S}_{1} \stackrel{\mu}{\rightarrow} \mathscr{I}_{3} \rightarrow 0 .
$$

In other words, $\mathscr{K}_{1}$ is the sheaf of linear syzygies. Let $U$ be an open subset of $G_{0}^{\prime}$ such that $\left.\Phi_{2}\right|_{U}$ is free. Denote generators of $\Phi_{2}(U)$ by $q_{1}, \ldots, q_{7}$ and thus we have

$$
\mathscr{K}_{1}(U)=\left\{\left(\sum_{i=1}^{7} q_{i} \otimes l_{i}\right) \mid l_{i} \in \mathscr{I}_{1}(U), \sum q_{i} l_{i}=0 \in \mathscr{I}_{3}\right\} .
$$

To simplify notation in the following lemma we write $\mathcal{H}_{\mathrm{om}}$ to denote $\mathcal{H}_{o m_{\mathrm{G}_{0}^{\prime}}}$.

Lemma 5.11. (1) On $G_{0}^{\prime}$ there is a morphism of locally free sheaves of ranks 28 and 24 respectively:

$$
h: \mathscr{H o m}\left(\mathscr{I}_{2}, \mathscr{Y}_{1}\right) \rightarrow \mathscr{H o m}\left(\mathscr{K}_{1}, \mathscr{Y}_{2} / \mathscr{I}_{2}\right),
$$

such that for any $I \in G_{0}^{\prime}$, we have $\operatorname{ker}(h \otimes k(I))=\operatorname{Hom}(I, S / I)_{-1}$.

(2) There is a locally free subsheaf of rank four $\mathcal{T} \subset \operatorname{ker}(h)$ inducing a morphism:

$$
\bar{h}: \operatorname{Hom}\left(\mathscr{I}_{2}, \mathscr{Y}_{1}\right) / \mathscr{T} \rightarrow \operatorname{Hom}\left(\mathscr{K}_{1}, \mathscr{Y}_{2} / \mathscr{I}_{2}\right),
$$

such that $\operatorname{ker}(\bar{h} \otimes k(I)) \neq 0$ if and only if $\operatorname{dim}_{k} \operatorname{Hom}(I, S / I)_{-1} \geq 5$.

Proof. (1) We have a map of locally free $\mathscr{O}_{G_{0}^{\prime}}$-modules: $\mathscr{K}_{1} \rightarrow \mathscr{I}_{2} \otimes \mathscr{S}_{1}$. This induces the map $\mathscr{K}_{1} \otimes \check{\mathscr{Y}}_{1} \rightarrow \mathscr{I}_{2}$. Applying $\mathscr{H}$ om $\left(-, \mathscr{Y}_{1}\right)$ to both sides we get

$$
\mathscr{H o m}\left(\mathscr{I}_{2}, \mathscr{S}_{1}\right) \rightarrow \operatorname{Hom}\left(\mathscr{K}_{1} \otimes \check{\mathscr{S}}_{1}, \mathscr{Y}_{1}\right) \cong \mathscr{H} \text { om }\left(\mathscr{K}_{1}, \mathscr{Y}_{1} \otimes \mathscr{S}_{1}\right) .
$$

For the isomorphism above, we are using identities about Hom, tensor product of sheaves, and sheaf duality from [Hartshorne 1977, p. 123]. The sequence $\mathscr{S}_{1} \otimes$ $\mathscr{S}_{1} \rightarrow \mathscr{S}_{2} \rightarrow \mathscr{Y}_{2} / \mathscr{I}_{2}$ gives a map from $\mathscr{H}$ om $\left(\mathscr{K}_{1}, \mathscr{Y}_{1} \otimes \mathscr{S}_{1}\right) \rightarrow \mathscr{H}$ om $\left(\mathscr{K}_{1}, \mathscr{Y}_{2} / \mathscr{I}_{2}\right)$. By composition we obtain the desired map:

$$
h: \mathscr{H o m}\left(\mathscr{I}_{2}, \mathscr{Y}_{1}\right) \rightarrow \mathscr{H o m}\left(\mathscr{K}_{1}, \mathscr{Y}_{2} / \mathscr{I}_{2}\right) .
$$


Let us take a moment and consider $h$ in concrete terms, since this will be used for proving part (2) of the lemma. Let $U \subset G_{0}^{\prime}$ be an open subset such that all relevant locally free sheaves are in fact free. Let $q_{1}, \ldots, q_{7}$ be the generators of $\Phi_{2}(U)$ and let $\sigma_{j}:=\sum_{i=1}^{7} q_{i} \otimes l_{i j}$ for $1 \leq j \leq 8$ be the generators of $\mathscr{K}_{1}(U)$. Finally, let $\phi \in \operatorname{Hom}\left(\mathscr{I}_{2}, \mathscr{I}_{1}\right)$ be a map $\left(q_{i} \mapsto m_{i}\right)$. Then $h(\phi)$ is the map

$$
\sigma_{j} \mapsto \sum \overline{m_{i} l_{i}}
$$

where $\overline{m_{i} l_{i}}$ is the reduction of $m_{i} l_{i}$ modulo $\Phi_{2}$.

(2) Over any $U$ where $\Phi_{2}$ is free, let $q_{1}, \ldots, q_{7}$ the global generators. Then we define $t_{1}: q_{i} \mapsto \frac{\partial}{\partial x_{1}} q_{i}$, and we define $t_{2}, t_{3}, t_{4}$ similarly. This defines a locally free subsheaf $\mathscr{T}(U):=\left\langle t_{1}, \ldots, t_{4}\right\rangle \subset \mathscr{H} \operatorname{lom}\left(\mathscr{I}_{2}, \mathscr{I}_{1}\right)$ of rank 4. By the proof of Lemma 5.10, the injection $\mathscr{T} \rightarrow \mathscr{H o m}\left(\mathscr{I}_{2}, \mathscr{S}_{1}\right)$ remains exact under pullback to a point. It follows that the quotient $\operatorname{Hom}\left(\mathscr{I}_{2}, \mathscr{S}_{1}\right) / \mathscr{T}$ is locally free of rank 24 [Hartshorne 1977, Ex II.5.8].

It remains to show that $\mathscr{T} \subset \operatorname{ker}(h)$ and that $\operatorname{ker}(\bar{h} \otimes k(I))$ is nontrivial if and only if $\operatorname{dim}_{k} \operatorname{Hom}(I, S / I)_{1} \geq 5$. This is immediate from the discussion preceding this theorem.

By the previous lemma, $\bar{h}$ is a map between locally free sheaves of rank 24 , and thus $\operatorname{det}(\bar{h})$ defines a divisor on $G_{0}^{\prime}$. To ensure that this is the restriction of a unique divisor on $G_{0}$, we need to verify that $Z_{1}$ and $Z_{2}$ are not too large. For this, we construct the rational curve $\tau: \mathbb{P}^{1} \rightarrow G_{0}$ defined for $t \neq \infty$ by

$$
I_{t}=\left(x_{1}^{2}, x_{2}^{2}, x_{3}^{2}, x_{4}^{2}, x_{1} x_{2}, x_{2} x_{3}+t x_{3} x_{4}, x_{1} x_{4}+t x_{3} x_{4}\right) .
$$

Lemma 5.12. $Z_{1} \cup Z_{2}$ is a closed set of codimension at least 2 in $G_{0}$.

Proof. $Z_{1}$ is closed because it is the support of the cokernel of the multiplication map $\mathscr{I}_{2} \otimes \mathscr{S}_{1} \rightarrow \mathscr{S}_{3}$. The intersection $Z_{2} \cap G_{0}^{\prime}$ is the degeneracy locus of

$$
\mathscr{H o m}\left(\mathscr{I}_{2}, \mathscr{Y}_{0}\right) \rightarrow \mathscr{H o m}\left(\mathscr{K}_{1}, \mathscr{Y}_{1}\right) \oplus \mathscr{H o m}\left(\mathscr{K}_{2}, \mathscr{S}_{2} / I_{2}\right),
$$

which is the analogue of Lemma 5.11 (1) for computing $\operatorname{Hom}\left(I_{2}, S_{0}\right)$. Thus $Z_{1} \cup Z_{2}$ is closed in $G_{0}$.

Because $\operatorname{Pic}\left(G_{0}\right)=\mathbb{Z}$ and $G_{0}$ is projective, checking that the 1-cycle $\tau$ does not intersect $Z_{1} \cup Z_{2}$ will show that $Z_{1} \cup Z_{2}$ has codimension at least 2 . By passing to the algebraic closure, we can assume that $k$ is algebraically closed. The group $k^{\times}$ acts on $\mathbb{A}^{4}$ by $\alpha \cdot\left(x_{1}, x_{2}, x_{3}, x_{4}\right)=\left(x_{1}, x_{2}, \alpha x_{3}, \alpha x_{4}\right)$, and taking $\alpha=t$ maps $I_{1}$ to $I_{t}$, for any $t$ other than 0 or $\infty$. Thus, it suffices to check that the following three 
ideals do not intersect $Z_{1} \cup Z_{2}$ :

$$
\begin{aligned}
I_{0} & =\left(x_{1}^{2}, x_{2}^{2}, x_{3}^{2}, x_{4}^{2}, x_{1} x_{2}, x_{2} x_{3}, x_{1} x_{4}\right), \\
I_{1} & =\left(x_{1}^{2}, x_{2}^{2}, x_{3}^{2}, x_{4}^{2}, x_{1} x_{2}, x_{2} x_{3}+x_{3} x_{4}, x_{1} x_{4}+x_{3} x_{4}\right), \\
I_{\infty} & =\left(x_{1}^{2}, x_{2}^{2}, x_{3}^{2}, x_{4}^{2}, x_{1} x_{2}, x_{2} x_{3}-x_{1} x_{4}, x_{3} x_{4}\right) .
\end{aligned}
$$

It is obvious that these are generated in degree 2. A change of variables transforms $I_{\infty}$ to the ideal $J$ from Proposition 5.1, which is smooth, so $\operatorname{Hom}\left(I_{t}, S / I_{t}\right)_{-2}=0$ for $t=1, \infty$. One can also check that $\operatorname{Hom}\left(I_{0}, S / I_{0}\right)_{-2}=0$, which holds in all characteristics because $I_{0}$ is a monomial ideal. Therefore, $Z_{1} \cup Z_{2}$ has codimension at least 2 .

Lemma 5.13. Let $D$ be the divisor on $G_{0}$ defined locally by $\operatorname{det}(\bar{h})$. Then $W_{0}$ belongs to the support of $D$.

Proof. The Hilbert scheme is singular on $W_{0}$, so $W_{0} \cap G_{0}^{\prime} \subset V(\operatorname{det}(\bar{h}))$. Since $W_{0}$ is a divisor, Lemma 5.12 tells us that $W_{0}$ intersects $G_{0}^{\prime}$, so the irreducibility of $W_{0}$ means that it is contained in $D$.

5D. An equation for $W_{0}$. In this section, except for the last paragraph, we restrict to the case $k=\mathbb{C}$ in order to use the representation theory of $\mathrm{GL}_{4}(\mathbb{C})$.

We will use the result of Lemma 5.13 to give an upper bound on the degree of $W_{0}$ in terms of Plücker coordinates. This leads to a proof of Theorem 1.3 over $\mathbb{C}$. The restriction to $\mathbb{C}$ will be removed in the next section.

Let $H$ be an effective divisor which generates $\operatorname{Pic}\left(G_{0}\right)=\mathbb{Z}$. First we compute the degree of $D$ in Plücker coordinates, using the rational curve $\tau$.

Lemma 5.14. The curve $\tau$ has intersection multiplicities

$$
\tau \cdot H=1 \text { and } \tau \cdot D=16 .
$$

Proof. For the first statement, let $p_{1}$ and $p_{2}$ be the Plücker coordinates corresponding to the $\left(x_{1}^{2}, x_{2}^{2}, x_{3}^{2}, x_{4}^{2}, x_{1} x_{2}, x_{2} x_{3}, x_{3} x_{4}\right)$ - and $\left(x_{1}^{2}, x_{2}^{2}, x_{3}^{2}, x_{4}^{2}, x_{1} x_{2}, x_{2} x_{3}, x_{1} x_{4}\right)$ minors respectively. Then $L=V\left(p_{1}\right)$ does not meet $I_{t}$ at infinity. For $t \neq \infty$, we see that $p_{2}\left(I_{t}\right) \neq 0$, so local equations for $L$ valid at all common points of $L$ and $\tau$ are given by $L=\frac{p_{1}}{p_{2}}$. Since this equation pulls back to $t$ on $\mathbb{P}^{1}-\infty$ the statement follows.

For the second statement, note from the proof of Lemma 5.12 that $I_{\infty}$ is a smooth point and $\tau$ does not intersect $Z_{1}$ or $Z_{2}$. Therefore, it suffices to check the degree on the open affine defined by $t \neq \infty$.

For every $t \neq \infty, I_{t}$ has the following 8 linear syzygies, where $q_{1}, \ldots, q_{7}$ are the generators of $I_{t}$ in the order in Equation (5-2). 


$$
\begin{array}{l|l}
\sigma_{1}=x_{2} q_{1}-x_{1} q_{5} & \sigma_{2}=x_{4} q_{1}-x_{1} q_{7}+t x_{3} q_{7}-t^{2} x_{4} q_{3} \\
\sigma_{3}=x_{1} q_{2}-x_{2} q_{5} & \sigma_{4}=x_{3} q_{2}-x_{2} q_{6}+t x_{4} q_{6}-t^{2} x_{3} q_{4} \\
\sigma_{5}=x_{2} q_{3}-x_{3} q_{6}+t x_{4} q_{3} & \sigma_{6}=x_{1} q_{4}-x_{4} q_{7}+t x_{3} q_{4} \\
\sigma_{7}=x_{3} q_{5}-x_{1} q_{6}+t x_{3} q_{7}-t^{2} x_{4} q_{3} & \sigma_{8}=x_{4} q_{5}-x_{2} q_{7}+t x_{4} q_{6}-t^{2} x_{3} q_{4}
\end{array}
$$

The intersection number $\tau \cdot D$ equals the degree of $\tau^{*}(\operatorname{det}(\bar{h}))$, which we compute by writing out $\tau^{*}(h)$ as a matrix. Let $\phi \in \operatorname{Hom}(I, S / I)_{-1}$ be written as $\phi\left(q_{i}\right)=$ $c_{i, 1} x_{1}+c_{i, 2} x_{2}+c_{i, 3} x_{3}+c_{i, 4} x_{4}$ and recall that, if $\sigma_{j}=\sum_{i} q_{i} \otimes l_{i j}$ then $h(\phi)\left(\sigma_{j}\right)=$ $\sum \overline{\phi\left(q_{i}\right) l_{i j}}$ where the bar indicates that we are considering the image as an element of $S_{2} / I_{2}$. The monomials $x_{1} x_{3}, x_{2} x_{4}$ and $x_{1} x_{4}$ are a basis of $S_{2} / I_{2}$ for $t \neq \infty$, so we can explicitly express the $h(\phi)\left(\sigma_{j}\right)$ as follows:

$$
\begin{array}{l|l}
h(\phi)\left(\sigma_{1}\right) & -c_{5,3} x_{1} x_{3}+c_{1,4} x_{2} x_{4}+\left(-t c_{1,3}+t c_{5,4}\right) x_{3} x_{4} \\
h(\phi)\left(\sigma_{2}\right) & \left(t c_{7,1}-c_{7,3}\right) x_{1} x_{3}+\left(c_{1,2}-t^{2} c_{3,2}\right) x_{2} x_{4}+ \\
& \left(-t c_{1,1}+c_{1,3}+t^{3} c_{3,1}-t^{2} c_{3,3}-t^{2} c_{7,2}+2 t c_{7,4}\right) x_{3} x_{4} \\
h(\phi)\left(\sigma_{3}\right) & c_{2,3} x_{1} x_{3}-c_{5,4} x_{2} x_{4}+\left(-t c_{2,4}+t c_{5,3}\right) x_{3} x_{4} \\
h(\phi)\left(\sigma_{4}\right) & \left(c_{2,1}-t^{2} c_{4,1}\right) x_{1} x_{3}+\left(t c_{6,2}-c_{6,4}\right) x_{2} x_{4}+ \\
& \left(-t c_{2,2}+c_{2,4}+t^{3} c_{4,2}-t^{2} c_{4,4}-t^{2} c_{6,1}+2 t c 6 z\right) x_{3} x_{4} \\
h(\phi)\left(\sigma_{5}\right) & -c_{6,1} x_{1} x_{3}+\left(t c_{3,2}+c_{3,4}\right) x_{2} x_{4}+\left(-t^{2} c_{3,1}+t c_{6,2}-c_{6,4}\right) x_{3} x_{4} \\
h(\phi)\left(\sigma_{6}\right) & \left(t c_{4,1}+c_{4,3}\right) x_{1} x_{3}-c_{7,2} x_{2} x_{4}+\left(-t^{2} c_{4,2}+t c_{7,1}-c_{7,3}\right) x_{3} x_{4} \\
h(\phi)\left(\sigma_{7}\right) & \left(c_{5,1}-c_{6,3}+t c_{7,1}\right) x_{1} x_{3}-t^{2} c_{3,2} x_{2} x_{4}+ \\
& \left(t^{3} c_{3,1}-t^{2} c_{3,3}-t c_{5,2}+c_{5,4}+t c_{6,4}-t^{2} c_{7,2}+t c_{7,4}\right) x_{3} x_{4} \\
h(\phi)\left(\sigma_{8}\right) & -t^{2} c_{4,1} x_{1} x_{3}+\left(c_{5,2}+t c_{6,2}-c_{7,4}\right) x_{2} x_{4}+ \\
& \left(t^{3} c_{4,2}-t^{2} c_{4,4}-t c_{5,1}+c_{5,3}-t^{2} c_{6,1}+t c_{6,3}+t c_{7,3}\right) x_{3} x_{4}
\end{array}
$$

Each row of the above lines yields three linear equations so $\tau^{*}(h)$ is represented by a $24 \times 28$ matrix $M$ as expected. Computation in Macaulay2 [Grayson and Stillman] shows that the ideal of $24 \times 24$ minors of $M$ is $\left(t^{16}\right)$ and the statement follows.

Corollary 5.15. The divisor $D$ is linearly equivalent to $16 H$.

The following lemma allows us to determine the degree of $W_{0}$.

Lemma 5.16. The divisor $D$ vanishes with multiplicity at least 8 on $W_{0}$.

Proof. By Lemma 5.13, we know that $\left|W_{0}\right| \subseteq|D|$. By Lemma 5.12, a general point of $W_{0}$ does not belong to $Z_{1} \cup Z_{2}$. Let $I$ be any such point. Since $I$ is a singular point in $R_{8}^{4}, I$ has tangent space dimension at least $\operatorname{dim}\left(R_{8}^{4}\right)+1=33$, and so the null space of $\bar{h} \otimes k(I)$ must have dimension at least 8 .

Choose 8 vectors from the null space as basis vectors, and any other 16 to complete a basis of the source of $\bar{h} \otimes k(I)$. This basis in the quotient ring lifts to a basis in the local ring $O_{G_{0}^{\prime}, I}$. When we represent the localization of the map 
$(\bar{h})_{I}$ as a matrix with respect to this basis we see that the first 8 columns belong to the maximal ideal $\mathfrak{m}_{I}$ of $\mathcal{O}_{G_{0}^{\prime}, I}$. Thus $\operatorname{det}(\bar{h})$ belongs to $\mathfrak{m}_{I}^{8}$, and in turn $D$ has multiplicity at least 8 at $I$.

Lemma 5.17. The ideal sheaf of $D$ is $\left(P^{8}\right)$, where $P$ is the Salmon-Turnbull Pfaffian.

Proof. Since $D$ is a divisor on $G_{0}$ its defining ideal in the homogeneous coordinate ring of the Plücker embedding of $G_{0}$ is generated by a single element $f$ of degree 16 in the Plücker coordinates. If $g$ is the square-free part of $f$ then Lemma 5.16 shows that $g$ has degree at most 2. Since $D$ is invariant under linear changes of variables, it follows from Lemmas 5.7 and 5.8 that $g=P$ and $f=P^{8}$.

By combining Lemmas 5.4, 5.13, and 5.17 we have now proven our descriptions of $W_{0}$ and $W$ :

Theorem 5.18. The subscheme $W_{0}$ is defined by $P$.

For the rest of the section, we return to the case that $k$ is a field, not necessarily algebraically closed, of characteristic not 2 or 3 .

Recall that if $M_{\lambda}$ is any monomial ideal in $G_{0}$ then there are local coordinates $c_{m^{\prime}}^{m}$ on $U_{\lambda} \cap H_{8}^{4}$. Moreover there is a surjection $\pi: R_{8}^{4} \cap U_{\lambda} \rightarrow W_{0} \cap U_{\lambda}$, and there is a rational map $\phi:\left(\mathbb{A}^{4}\right)^{8} / / S_{8} \rightarrow R_{8}^{4} \cap U_{\lambda}$ given by $c_{m^{\prime}}^{m}=\frac{\Delta_{\lambda-m^{\prime}+m}}{\Delta_{\lambda}}$ whose image is dense in $R_{8}^{4} \cap U_{\lambda}$.

Lemma 5.19. With $U_{\lambda}$ as above, the function $P \circ \pi$ vanishes identically on $R_{8}^{4} \cap U_{\lambda}$ over an arbitrary field $k$.

Proof. The composition $P \circ \pi \circ \phi$ is a rational function with integer coefficients. Theorem 5.18 proves that $P \circ \pi \circ \phi=0$ in $\mathbb{C}\left[q_{i}^{(j)}\right]\left[\Delta_{\lambda}^{-1}\right]$. Therefore, $P \circ \pi \circ \phi=0$ in $\mathbb{Z}\left[q_{i}^{(j)}\right]\left[\Delta_{\lambda}^{-1}\right]$.

Theorem 5.20. The following irreducible subsets of $G_{0}$ coincide:

(1) $W_{0}$;

(2) $V(P)$, the vanishing of the pullback to $G$ of the Salmon-Turnbull Pfaffian;

(3) the homogeneous ideals with Hilbert function $(1,4,3)$ which are flat limits of ideals of distinct points.

As a consequence, if we let $\left.\pi\right|_{G}: G \rightarrow G_{0}$ be the restriction of the projection from Lemma 5.4 then $W=V\left(\left.P \circ \pi\right|_{G}\right)$.

Proof. For other fields, note that for the ideal $J$ of Proposition 5.1 with $d=4$, we have that $P \circ \pi(J)=P(J)=1$ and thus $P \circ \pi$ does not vanish uniformly on $G$ in any characteristic. By the previous lemma, $P \circ \pi$ vanishes uniformly on $R_{8}^{4}$ for any $k$. Thus $W \subseteq V\left(\left.P \circ \pi\right|_{G}\right)$. As both $W$ and $V\left(\left.P \circ \pi\right|_{G}\right)$ are integral closed subschemes of codimension 1 in $G$, they are equal. 


\section{Proofs of main results}

In this section, $k$ denotes a field of characteristic not 2 or 3 . We have used our characteristic assumption in order to apply the theory of duality in Sections 3 and 4 and to define the trace map of Lemma 5.3.

Proof of Theorem 1.1. The irreducibility of $H_{n}^{d}$ when $d$ is at most 3 or $n$ is at most 7 follows for an algebraically closed field from Theorem 4.20. For a nonalgebraically closed field, the Hilbert scheme is irreducible because it is irreducible after passing to the algebraic closure. Proposition 3.1 and the same argument as in Lemma 5.3 show that when $d$ is at least $4, G_{8}^{d}$ is irreducible and $(8 d-7)$-dimensional, and Proposition 5.1 shows that it is a separate component. Theorem 4.20 shows that there are no other components, again, by passing to the algebraic closure if necessary.

Proof of Theorem 1.2. This follows from Theorem 3.9.

Proof of Theorem 1.3. The statement that $R_{8}^{4} \cap G_{8}^{4}$ is a prime divisor on $G_{8}^{4}$ is proved in Lemma 5.4. The equivalence of the set-theoretic description and the local equation description follows from Lemma 5.6. Theorem 5.20 proves that the Salmon-Turnbull Pfaffian is the correct local equation.

Proof of Theorem 1.4. Let $M_{\lambda}$ be some monomial ideal and consider the monomial chart $U_{\lambda}$. If $M_{\lambda}$ does not have Hilbert function $(1,4,3)$ then $U_{\lambda} \cap G_{8}^{4}=\varnothing$ so that the zero ideals will cut out $R_{8}^{4}$. If $M_{\lambda}$ has Hilbert function $(1,4,3)$, then Lemma 5.19 and Theorem 5.20 show that the zero set of the pullback of the Pfaffian is precisely $R_{8}^{4} \cap U_{\lambda}$.

\section{Open questions}

The motivating goal behind this work is understanding the smoothable component of the Hilbert scheme as explicitly as possible, and not just as the closure of a certain set. This can be phrased more abstractly by asking what functor the smoothable component represents, or, more concretely, by describing those algebras which occur in the smoothable component. In this paper we have done the latter for $n$ at most 8 . The following are natural further questions to ask:

- For $d$ greater than 4, which algebras with Hilbert function $(1, d, 3)$ are smoothable? Generically, such algebras are not smoothable. Computer experiments lead us to conjecture that, for smoothable algebras, the analogue of the skew symmetric matrix in Theorem 1.3 has rank at most $2 d+2$. However, a dimension count shows that this rank condition alone is not sufficient for such an algebra to be smoothable. What are the other conditions? 
- What is the smallest $n$ such that $H_{n}^{3}$ is reducible? We have shown $H_{8}^{3}$ is irreducible and Iarrobino [1985, Example 3] has shown that $H_{78}^{3}$ is reducible.

- Is $H_{n}^{d}$ ever nonreduced? What is the smallest example? Does it ever have generically nonreduced components?

\section{Acknowledgments}

We thank David Eisenbud, Bjorn Poonen, and Bernd Sturmfels for many insightful discussions, guidance, and key suggestions. We would also like to thank Eric Babson, Jonah Blasiak, Mark Haiman, Anthony Iarrobino, Diane Maclagan, Scott Nollet, Greg Smith, and Mike Stillman for helpful conversations. In addition, we thank the referee for suggesting the simplified proof in Lemma 4.1. Macaulay2 [Grayson and Stillman] and Singular [Greuel et al. 2005] were used for experimentation.

\section{References}

[Evain 2004] L. Evain, "Irreducible components of the equivariant punctual Hilbert schemes", Adv. Math. 185:2 (2004), 328-346. MR 2005g:14009 Zbl 1064.14004

[Fogarty 1968] J. Fogarty, "Algebraic families on an algebraic surface", Amer. J. Math 90 (1968), 511-521. MR 38 \#5778 Zbl 0176.18401

[Grayson and Stillman] D. R. Grayson and M. E. Stillman, "Macaulay 2, a software system for research in algebraic geometry", Available at http://www.math.uiuc.edu/Macaulay2.

[Greuel et al. 2005] G.-M. Greuel, G. Pfister, and H. Schönemann, "Singular 3.0", a computer algebra system for polynomial computations, Centre for Computer Algebra, University of Kaiserslautern, 2005, Available at http://www.singular.uni-kl.de.

[Haiman 1998] M. Haiman, " $t, q$-Catalan numbers and the Hilbert scheme", Discrete Math. 193:1-3 (1998), 201-224. MR 2000k:05264 Zbl 1061.05509

[Haiman and Sturmfels 2004] M. Haiman and B. Sturmfels, "Multigraded Hilbert schemes", J. Algebraic Geom. 13:4 (2004), 725-769. MR 2005d:14006 Zbl 1072.14007

[Harris 1992] J. Harris, Algebraic geometry: A first course, Graduate Texts in Mathematics 133, Springer, New York, 1992. MR 93j:14001 Zbl 0779.14001

[Hartshorne 1977] R. Hartshorne, Algebraic geometry, Graduate Texts in Mathematics 52, Springer, New York, 1977. MR 57 \#3116 Zbl 0367.14001

[Iarrobino 1972] A. Iarrobino, "Reducibility of the families of 0-dimensional schemes on a variety", Invent. Math. 15 (1972), 72-77. MR 46 \#170 Zbl 0227.14006

[Iarrobino 1985] A. Iarrobino, Jr., "Compressed algebras and components of the punctual Hilbert scheme", pp. 146-165 in Algebraic geometry, Sitges (Barcelona, 1983), edited by E. Casas-Alvero et al., Lecture Notes in Math. 1124, Springer, Berlin, 1985. MR 86k:14001 Zbl 0567.14001

[Iarrobino and Emsalem 1978] A. Iarrobino and J. Emsalem, "Some zero-dimensional generic singularities; finite algebras having small tangent space", Compositio Math. 36:2 (1978), 145-188. MR 81c:14004 Zbl 0393.14002

[Mazzola 1980] G. Mazzola, "Generic finite schemes and Hochschild cocycles", Comment. Math. Helv. 55:2 (1980), 267-293. MR 82k:14010 Zbl 0463.14004 
[Miller and Sturmfels 2005] E. Miller and B. Sturmfels, Combinatorial commutative algebra, Graduate Texts in Mathematics 227, Springer, New York, 2005. MR 2006d:13001 Zbl 1090.13001

[Poonen 2008] B. Poonen, "Isomorphism types of commutative algebras of finite rank over an algebraically closed field", pp. 111-120 in Computational arithmetic geometry, edited by K. E. Lauter and K. A. Ribet, Contemp. Math. 463, Amer. Math. Soc., Providence, RI, 2008. MR 2459993 Zbl 1155.13015

[Salmon 1874] G. Salmon, A treatise on the analytic geometry of three dimensions, Hodges, Foster, London, 1874. MR 20\#1265

[Turnbull 1922] H. W. Turnbull, "The invariant theory of three quadrics", Proc. London Math. Soc. (2) 20:1 (1922), 465-489.

Communicated by Hubert Flenner

Received 2008-06-27 Revised 2009-04-23 Accepted 2009-06-30

dustin@math.berkeley.edu Department of Mathematics, University of California, Berkeley, CA 94720, United States

derman@math.berkeley.edu Department of Mathematics, University of California, Berkeley, CA 94720, United States

velasco@math.berkeley.edu Department of Mathematics, University of California, Berkeley, CA 94720, United States

bviray@math.berkeley.edu Department of Mathematics, University of California, Berkeley, CA 94720, United States 


\title{
Discretely ordered groups
}

\author{
Peter A Linnell, Akbar Rhemtulla and Dale P. O. Rolfsen
}

\begin{abstract}
We consider group orders and right-orders which are discrete, meaning there is a least element which is greater than the identity. We note that nonabelian free groups cannot be given discrete orders, although they do have right-orders which are discrete. More generally, we give necessary and sufficient conditions that a given orderable group can be endowed with a discrete order. In particular, every orderable group $G$ embeds in a discretely orderable group. We also consider conditions on right-orderable groups to be discretely right-orderable. Finally, we discuss a number of illustrative examples involving discrete orderability, including the Artin braid groups and Bergman's nonlocally-indicable right orderable groups.
\end{abstract}

\section{Introduction}

Let $G$ be a group and suppose < is a strict total order relation on the set of its elements. Then $(G,<)$ is a right-ordered group if $f<g$ implies that $f h<g h$ for all $f, g, h \in G$. If in addition $f<g$ implies that $h f<h g$, then we say $(G,<)$ is an ordered group. If such an order exists for a given group $G$, we say that $G$ is right-orderable or orderable, respectively. We call the order $<$ discrete if there is an element $a \in G$ such that $1<a$, where 1 denotes the identity element of $G$, and there is no element of $G$ strictly between these.

For a right-ordered group, the positive cone $P:=\{g \in G \mid 1<g\}$ satisfies

(1) $P$ is closed under multiplication and

(2) for every $g \in G$, exactly one of $g=1, g \in P$ or $g^{-1} \in P$ holds.

Conversely, if a group $G$ has a subset $P$ with properties (1) and (2), it is routine to verify that the order defined by $g<h$ if and only if $h g^{-1} \in P$ makes $(G,<)$ a right-ordered group. Similarly, a group $G$ is orderable if and only if it admits a subset $P$ satisfying (1), (2) and

(3) $g P g^{-1}=P$ for all $g \in G$.

MSC2000: primary 20F60; secondary 06F15, $20 \mathrm{~F} 36$.

Keywords: discrete order. 
A subset $X$ of a (right-) ordered group $G$ is convex if $x<y<z$ and $x, z \in X$ imply $y \in X$. We recall that the set of all convex subgroups of a (right-) ordered group is linearly ordered by inclusion. A convex jump $C \longmapsto D$ is a pair of distinct convex subgroups such that $C \subset D$ and there are no convex subgroups strictly between them. In particular, the convex jump determined by a nonidentity element $g$ of $G$ is defined by $C=$ the union of all convex subgroups not containing $g$ and $D=$ the intersection of all convex subgroups which do contain $g$. If the group is orderable, then for any convex jump $C \longmapsto D, C$ is normal in $D$ and the quotient $D / C$ embeds in $\mathbb{R}$, the additive reals, by an order-preserving isomorphism.

Lemma 1.1. If $<$ is a discrete right-order on $G$ and $a$ is the least positive element under $<$, then $\langle a\rangle$ is convex. Moreover, for any $g \in G$, we have $a^{-1} g<g<a g$ and there is no element strictly between these elements of $G$. If the right-order $<$ is not discrete, then it is dense in the sense that for any $f, g \in G$ with $f<g$, there exists $h \in G$ with $f<h<g$.

Proof. Since $a^{-1}<1<a$, we see that $a^{-1} g<g<a g$. If $g<x<a g$, then $1<x g^{-1}<a$, a contradiction. Hence there is no element strictly between $g$ and $a g$. Similarly there is no element between $a^{-1} g$ and $g$. In particular, for any integers $n<m, a^{n}<g<a^{m}$ implies $g \in\langle a\rangle$ and thus $\langle a\rangle$ is convex. If there exists $f<g$ with no element strictly between, a routine calculation shows $g f^{-1}$ is the least positive element for the order.

Note that according to our definitions, the trivial group 1 has exactly one right order, and this order is dense but not discrete.

Situated strictly between the class of right-orderable groups and the class of orderable groups is the class of locally indicable groups. Recall that a group $G$ is locally indicable if every finitely generated nontrivial subgroup of $G$ has an infinite cyclic quotient. Such groups are right-orderable, as was shown by Burns and Hale [1972]. On the other hand, a right-orderable group need not be locally indicable as was shown by Bergman [1991]. However for a large class of groups the class of right-orderable groups coincides with the class of locally indicable groups. Further results on this topic are contained in [Linnell 2001], [Longobardi et al. 2000], and especially in [Morris 2006].

Our interest in considering locally indicable groups $G$ is due to the fact that such groups have a series (defined below) with torsion-free abelian factors as shown in [Brodskiı 1984]. They possess a right-order in which the set of convex subgroups form a series with factors which are order isomorphic to subgroups of the additive group of reals; we shall refer to such orders as lexicographic. (Such orders are also called Conrad orders and are characterized by the condition that if $g>1$ and $h>1$, then there exists a positive integer $n$ such that $(g h)^{n}>h g$; see [Botto Mura and Rhemtulla 1977, §7.4] for further details.) Note that for such an ordering, any 
nontrivial element $g \in G$ is positive if and only if the cosets satisfy $C g>C$ in the factor group $D / C$ determined by $g$.

By a series for $G$ we mean a set $\Sigma=\left\{H_{\lambda} \mid \lambda \in \Lambda\right\}$ of subgroups of $G$, where $\Lambda$ is a totally ordered set of indices, satisfying:

- if $\lambda<\mu$ then $H_{\lambda} \subset H_{\mu}$,

- $\{1\}$ and $G$ belong to $\Sigma$,

- $\Sigma$ is closed under arbitrary unions and intersections,

- if $\mu$ immediately follows $\lambda$ in $\Lambda$, then $H_{\lambda}$ is normal in $H_{\mu}$ and $H_{\mu} / H_{\lambda}$ is called a factor associated to the jump $H_{\lambda} \longmapsto H_{\mu}$.

In the next section we characterize groups that have discrete orders. We show that a group $G$ has a discrete order if and only if it is an orderable group and its center $Z(G)$ contains an isolated infinite cyclic group. Recall that a subgroup $H$ of a group $G$ is said to be isolated if $g \in G$ and $g^{n} \in H$ for some $n>0$ implies $g \in H$.

In Section 3, we deal with groups possessing discrete lexicographic right-orders and discrete right-orders. It will follow, in particular, that any nontrivial finitely generated orderable group has discrete right-orders and if it has a central order (as is the case for free groups, pure braid groups and wreath products or free products of such groups), then it has discrete lexicographic right-orders. Recall that an order $<$ on $G$ is called central if for every convex jump $C \longmapsto D$, we have $[D, G] \subseteq C$ where $[D, G]$ denotes the subgroup $\left\langle d^{-1} g^{-1} d g \mid d \in D, g \in G\right\rangle$.

The result is of course not true for orderable groups in general. The additive group of rational numbers has no discrete right-order.

The final section presents examples of discretely ordered groups which have nontrivial subgroups (for example, the commutator subgroup) upon which the restriction of the given order is dense. We also note that there exist finitely generated right-orderable groups, for example, the Artin braid groups $B_{n}, n \geq 5$, that are not locally indicable, yet have a discrete right-order.

\section{Discrete orders}

Theorem 2.1. If $<$ is a discrete order on a group $G$, then there exists an element $z$ in the center $Z(G)$ such that $\langle z\rangle$ is convex under $\langle$ and $1 \longmapsto\langle z\rangle$ is a jump. Conversely, if $G$ is an orderable group and $Z(G)$ contains an isolated infinite cyclic group, then there is a discrete order on $G$.

Proof. Let $<$ be a discrete order on $G$ with $z>1$ as the minimal positive element. Then $g^{-1} z g$ is positive for every $g \in G$. Moreover, $z<g^{-1} z g$ implies $1<g z g^{-1}<z$, a contradiction. Thus $z \in Z(G)$. Also Lemma 1.1 shows that $\langle z\rangle$ is convex under $<$ and $1 \longmapsto\langle z\rangle$ is a jump. 
Conversely, let $\langle z\rangle$ be an isolated subgroup in the center $Z(G)$ of an orderable group $G$. Since $G$ is orderable, so is $G / Z(G)$; see [Botto Mura and Rhemtulla 1977, Theorem 2.2.4]. Moreover, $Z(G) /\langle z\rangle$ is orderable since $\langle z\rangle$ is isolated in $Z(G)$. Order $\langle z\rangle$ (with $z$ positive), $Z(G) /\langle z\rangle$ and $G / Z(G)$. Now order $G$ as follows. If $1 \neq g \in G \backslash Z(G)$, then put $g$ in the positive cone if $g Z(G)$ is positive; if $g \in Z(G) \backslash\langle z\rangle$, then put $g$ in the positive cone if $g\langle z\rangle$ is positive; if $g=z^{n}$, then put $g$ in the positive cone if $0<n$. It is routine to verify that this gives a discrete order on $G$ with $z$ as the minimal positive element.

Corollary 2.2. For any orderable group $G$, the group $\mathbb{Z} \times G$ has a discrete order. In particular, every orderable group embeds in a discretely orderable group, whose order extends the given order.

\section{Discrete right orders}

We begin this section with the following result which is easy to prove. It is not required in the proofs of the other results.

Lemma 3.1. If $(G,<)$ is a nontrivial right-ordered group such that the order $<i s$ a well order on the set of positive elements of $G$, then $G$ is infinite cyclic.

Lemma 3.2. If $<$ is a discrete right order on $G$ and a the least positive element under $<$ then for any element $1<g \in G$, we have $1<a g a^{-1}$ and $1<a^{-1} g a$.

Proof. Since $a \leq g, 1 \leq g a^{-1}$. Thus $a g a^{-1}$ is a product of two positive elements and hence positive. By Lemma 1.1, there is no element of $G$ strictly between $a^{-1} g$ and $g$. Since $1<g, 1 \leq a^{-1} g$ and so $a \leq a^{-1} g a$.

Definition 3.3. Let $<$ be a right order on a group $G, C$ a subgroup of $G$ and $a \in G$. We shall say that conjugation by a preserves order on $C$ to mean that $C$ is normalized by $\langle a\rangle$ and conjugation by $a$ and by $a^{-1}$ preserves the order on $(C,<)$.

Lemma 3.4. Suppose < is a right order on a group $G, C$ is a subgroup of $G$, $1 \neq a \in G$ and $C \cap\langle a\rangle=1$. If conjugation by a preserves order on $C$, then there is a discrete right order on the subgroup $\langle C, a\rangle$ with a as the minimal positive element. Moreover, this right order and the given right order agree on $C$. Finally if $a E a^{-1}=E$ for all convex subgroups $E$ of $C$, then the convex subgroups of $H$ under this new right order are $\{1\}$ and $\langle a, E\rangle$, where $E$ is a convex subgroup of $C$.

Proof. Set $H=\langle a, C\rangle$. An element $g \in H$ has a unique expression as $g=a^{n} c$ where $c \in C$ and $n \in \mathbb{Z}$. Define the set $P$ as follows: $g \in P$ if $1<c$ or $c=1$ and $n>0$. Note that $P \cup P^{-1}=H \backslash\{1\}$ and $P \cap P^{-1}=\varnothing$. Moreover if $g=a^{n} c$ and $h=a^{m} d$ are in $P$, then their product $g h=a^{n+m}\left(a^{-m} c a^{m}\right) d \in P$ as conjugation by $a^{m}$ preserves order on $C$. Thus the order $\prec$ on $H$ given by $g \prec h$ if and only if $h g^{-1} \in P$ is a right order on $H$. Furthermore if $g=a^{n} c$ and $h=a^{m} d$ are in $H$, 
then $g \prec h$ if and only if $c<d$ or $c=d$ and $n<m$. It is now clear that $a$ is the least positive element under this order, and that $<$ and $\prec$ agree on $C$. Finally we verify that the convex subgroups are $\langle a, E\rangle$, where $E$ is a convex subgroup of $C$.

Set $A=\langle a\rangle$, so $H=A C$. If $K$ is a nontrivial convex subgroup of $H$, then $a \in K$ and $C \cap K$ is a convex subgroup of $C$, and we have $(K \cap C) A=K \cap C A=K$. Thus $K=\langle a, E\rangle$ where $E=C \cap K$. On the other hand if $E$ is a convex subgroup of $C$, we claim that $A E$ is a convex subgroup of $H$. Suppose $a^{m} b \prec a^{n} c \prec a^{p} d \in E A$, where $b, d \in E, c \in C$, and $m, n, p \in \mathbb{Z}$. Then $b \leq c \leq d$ and hence $c \in E$, and it follows that $A E$ is a convex subgroup of $(H, \prec)$, as required.

Theorem 3.5. Let $(G,<)$ be an ordered group, $1 \neq a \in G$ and $C \longmapsto D$ the convex jump determined by a (thus $a \in D \backslash C$ and $D / C$ is torsion-free abelian). If $D /\langle a, C\rangle$ is torsion free, then there is a discrete right order on $G$ with a as the minimal positive element. Moreover, if also $[a, F] \subseteq E$ for every jump $E \longmapsto F$, then there is a discrete lexicographic right order on $G$ (with a as minimal positive element).

Proof. Set $H=\langle a, C\rangle$. The hypothesis of Lemma 3.4 applies and we right order $H$ as described there. Next we order the factor group $D / H$. This is possible since $D / H$ is torsion-free abelian. Define the set $Q \subset G$ as follows. If $g \in H$, then $g \in Q$ if $g$ is positive in the order on $H$ described above. If $g \in D \backslash H$, then put $g$ in $Q$ if $g H$ is positive in the order on $D / H$ given above. If $g \in G \backslash D$, then put $g$ in $Q$ if $g$ is positive in $(G,<)$, the original order on $G$.

It is routine to verify that $Q \cup Q^{-1}=G \backslash\{1\}, Q \cap Q^{-1}=\varnothing$ and $Q Q \subseteq Q$, thus giving a right order $\prec$ on $G$ with $Q=\{g \in G \mid 1 \prec g\}$.

The same right order $\prec$ is lexicographic if $[a, F] \subseteq E$ for every jump $E \longmapsto F \subseteq C$. The convex subgroups are $\{1\},\langle a\rangle$, and $\langle a, E\rangle$ for every subgroup $E$ convex under the original order $<$. This follows from Lemma 3.4: note that $E=\langle a, E\rangle$ if $E \geq D$ and $E \cap C$ is a convex subgroup of $C$.

Corollary 3.6. Nontrivial free groups have discrete lexicographic right orders.

Proof. This follows from the fact that the descending lower central series terminates in $\{1\}$ and the factors are free abelian groups. Thus any element may be made to be the least positive element so long as it is a primitive element of the factor group that is determined by the element.

Corollary 3.6 can be generalized to free partially commutative groups. These are described in [Duchamp and Krob 1992, §1.1], and the definition given there does not require these groups to be finitely generated. Free partially commutative groups are known under many other names, in particular they are also called right-angled Artin groups [Charney 2007], at least for finitely generated groups.

Corollary 3.7. Nontrivial free partially commutative groups have discrete lexicographic right orders. 
Proof. Free partially commutative groups are residually nilpotent by [Duchamp and Krob 1992, Theorem 2.3]. Furthermore [Duchamp and Krob 1992, Theorems $1.1,2.1]$ show that the quotients of the lower central series are free abelian groups. The result now follows from Theorem 3.5.

Note that a nonabelian free group does not have a discrete order. This follows from Theorem 2.1.

Finally, all surface groups (orientable or not) except the Klein bottle and projective plane are residually torsion-free nilpotent, by [Baumslag 1968, Theorem 1] (we would like to thank Warren Dicks for this reference). Thus these surface groups also have lexicographic discrete right orders. With the exception of the torus, these groups have trivial center and therefore do not enjoy discrete orders.

The pure braid groups $P_{n}$, like free groups and surface groups, are also residually torsion-free nilpotent. But, unlike those examples, the groups $P_{n}$ do have discrete orders. The center $Z\left(P_{n}\right)$ is infinite cyclic, generated by $z=$ the full twist braid (often denoted $\Delta_{n}^{2}$ ). Since $\langle z\rangle$ is trivially isolated in $Z\left(P_{n}\right)$, the second part of Theorem 2.1 provides a discrete order with $\Delta_{n}^{2}$ as least positive element. In fact any discrete order of $P_{n}$ must have $\Delta_{n}^{2}$ (or its inverse) as least positive element.

\section{Examples}

A group may have a lexicographic right order and not have any discrete right order even when the factors formed by the convex jumps are all infinite cyclic. One example of this is the following.

Example 4.1. Let $G=\left\langle a_{i} \mid i \in \mathbb{Z}\right\rangle$ with defining relations $\left[a_{i}, a_{j}\right]=1$ if $|i-j|>1$ and $a_{i+1} a_{i} a_{i+1}^{-1}=a_{i}^{-1}$.

Every right order on $G$ is lexicographic with the subgroups $\left\langle a_{i} \mid i<j\right\rangle$ forming the chain of convex subgroups, and every right order is determined by the $a_{i}$ (that is, whether or not $a_{i}$ is in the positive cone for each $i \in \mathbb{Z}$ ). This construction is just the expansion of the well known (Klein bottle) group $D=\langle a, b\rangle$ where $b^{-1} a b=a^{-1}$. There are exactly four right orders on $D$, every one discrete with $a$ or $a^{-1}$ as the minimal positive element.

We show next that any infinite cyclic extension of the group $G$ of this example has a discrete right order if it is finitely generated. However we can have a metacyclic extension of $G$ that is finitely generated and right orderable but without any discrete right order. These are given as Proposition 4.2 and Example 4.3. If $x, t$ are elements of a group, then $x^{t}$ will denote $t^{-1} x t$.

Proposition 4.2. Let $\Gamma=G\langle t\rangle$ be a finitely generated infinite cyclic extension of the group $G$ given in Example 4.1. Then $\Gamma$ has a discrete right order with $t$ (or $\left.t^{-1}\right)$ as the minimal positive element. 
Proof. Every nontrivial element $g \in G$ has unique expression of the form

$$
g=a_{r_{1}}^{d_{1}} \ldots a_{r_{k}}^{d_{k}}
$$

where $r_{1}<\cdots<r_{k}$ and $d_{i} \neq 0$ for all $i$. Call $a_{r_{k}}^{d_{k}}$ the leading term of $g$ and denote it by $\ell(g)$. Call $r_{k}$ the leading suffix of $g$.

Note that $\ell\left(g^{n}\right)=(\ell(g))^{n}$ for all $n \in \mathbb{Z} \backslash\{0\}$. Moreover, if $\ell(g)=a_{r}^{j}, \ell(h)=a_{s}^{k}$ and $r<s$, then $\ell(g h)=\ell(h g)=\ell(h)$. Since $\left(a_{i+1}^{t}\right)^{-1}\left(a_{i}^{t}\right)\left(a_{i+1}^{t}\right)=\left(a_{i}^{t}\right)^{-1}$, we see that the leading suffix of $\left(a_{i+1}\right)^{t}$ is greater than that of $\left(a_{i}\right)^{t}$ by at least one. Thus also the leading suffix of $\left(a_{i+1}\right)^{t^{-1}}$ is greater than that of $\left(a_{i}\right)^{t^{-1}}$ by at least one, and we deduce that the leading suffix of $\left(a_{i+1}\right)^{t}$ is greater than that of $\left(a_{i}\right)^{t}$ by exactly one. It follows that $i>j$ implies that the leading suffix of $\left(a_{i}\right)^{t}$ is greater than that of $\left(a_{j}\right)^{t}$ by exactly $i-j$.

Since $\Gamma$ is finitely generated, the leading suffix of $a_{i}^{t}$ (or that of $a_{i}^{t^{-1}}$ ) is greater than $i$ for at least one value of $i$ - otherwise $\left\langle a_{j} \mid j \leq i\right\rangle$ is normal in $\Gamma$ for every $i$, and hence $\Gamma$ can not be finitely generated. Thus if the leading suffix of $a_{0}^{t}$ is $n$, then the leading suffix of $a_{i}^{t}$ is $i+n$ for every integer $i$, and we may assume that $n>0$.

We now right order the group $G$ by putting $a_{0}, a_{1}, \ldots, a_{n-1}$ in the positive cone $P$. Next, for all $n \leq r<2 n$, we put $a_{r} \in P$ if the exponent of $\ell\left(a_{r-n}^{t}\right)$ is positive and $a_{r}^{-1} \in P$ otherwise. Next put $a_{r+n}$ or $a_{r+n}^{-1}$ in $P$ depending on whether the exponent of $\ell\left(\left(\ell\left(a_{r-n}\right)^{t}\right)^{t}\right)$ is positive or negative. Continue this process. For every integer $i \geq 0$ we have determined whether $a_{i}$ or its inverse is in $P$. Next, for $0>r \geq-n$ put $a_{r} \in P$ if the exponent of $\ell\left(a_{r+n}^{t^{-1}}\right)$ is positive. Put $a_{r}^{-1} \in P$ otherwise. Continue this process. This takes care of all $a_{i}$ for $i \in \mathbb{Z}$. Note that the above order on $G$ is $\langle t\rangle$ invariant. Hence by Lemma 3.4, $\Gamma$ has a right discrete order with $t$ as the minimal positive element.

Example 4.3. Let $G$ be the group in Example 4.1. Consider the map

$$
\phi:\left\{a_{i} \mid i \in \mathbb{Z}\right\} \rightarrow\left\{a_{i}^{-1} \mid i \in \mathbb{Z}\right\}
$$

given by $\phi\left(a_{i}\right)=a_{i}^{-1}$. Then $\phi$ extends uniquely to an automorphism of $G$ that inverts every $a_{i}$. Let $\langle G, u\rangle$ be the infinite cyclic extension of $G$ by $\langle u\rangle$ where $u^{-1} a_{i} u=a_{i}^{-1}$ for all $i \in \mathbb{Z}$. Note that $\langle G, u\rangle$ is right orderable because it is an infinite cyclic extension of the right orderable group $G$. However, it has no discrete right order. This can be seen as follows. Suppose $g \in G$ and $c:=g u^{j}$ is a minimal positive element under some right order $\langle$ on $\langle G, u\rangle$. Since $G$ has no discrete right order, $j \neq 0$ and it must be even otherwise $c^{-1} a_{i} c=a_{i}^{-1}$ for some $i$, contradicting Lemma 3.2.

Suppose $1<u$. Then $a_{i}^{r}<u$ for every $i, r \in \mathbb{Z}$, for if $1<u<a_{i}^{r}$, then $u a_{i}^{-r}<1$. Hence $a_{i}^{r}=u a_{i}^{-r} u^{-1}<1$, a contradiction. Thus $h<u$ for all $h \in G$. Since 
$1<c=g u^{j}=u^{j} g$, we see that $j$ is positive, and then we have $1<u^{j-1}, u g$, which contradicts the hypothesis that $c$ is the minimal positive element. The argument is similar if $u<1$. We note in particular that if $1<u$, then $h<u$ for every $h \in G$.

Next extend the group $\langle G, u\rangle$ by the infinite cyclic group $\langle v\rangle$ to get the group $J=\langle G, u, v\rangle$ where the action of $v$ under conjugation is as follows: $v^{-1} a_{i} v=a_{i+1}$, the shift automorphism, and $v^{-1} u v=u^{-1}$. Note that $\langle G, v\rangle=\left\langle a_{0}, v\right\rangle, J=\left\langle a_{0}, u, v\right\rangle$ and $J$ is right orderable.

We now show that $J$ has no discrete right order. Suppose that $c:=g u^{j} v^{k}$ is a minimal positive element under a right order $<$ on $J$. Then $k \neq 0$, since otherwise the restriction of the right order $<$ to $\langle G, u\rangle$ would be discrete with $g u^{j}$ as the minimal positive element. We have seen that this is not possible. Next note that $k$ must be even, otherwise assume without loss of generality that $u>1$. Then $c u c^{-1}=g u^{-1} g^{-1}<1$, which contradicts Lemma 3.2. Since $v u v^{-1}=u^{-1}$, we see that $1<v$ implies $x<v$ for every $x \in\langle G, u\rangle$, in particular $k>0$ and $g u^{j} v^{k-1}>1$. This contradicts the hypothesis that $c$ is the minimal positive element. Similarly we cannot have $1>v$, which finishes the verification that $J$ has no discrete right order.

It is possible for a discretely (right-) ordered group to have a subgroup on which the same order is dense. Indeed, by Corollary 2.2, any densely ordered group is a subgroup of a discretely ordered group, whose order extends the given order. Following is a natural example of this phenomenon for right-ordered groups.

Example 4.4. The Artin braid groups $B_{n}$ have a discrete right-order, which becomes dense when restricted to the commutator subgroup. For each integer $n \geq 2$, $B_{n}$ is the group generated by $\sigma_{1}, \sigma_{2}, \ldots, \sigma_{n-1}$, subject to the relations

$$
\sigma_{i} \sigma_{j}=\sigma_{j} \sigma_{i} \quad \text { if }|i-j|>1 \quad \text { and } \quad \sigma_{i} \sigma_{j} \sigma_{i}=\sigma_{j} \sigma_{i} \sigma_{j} \quad \text { if }|i-j|=1 .
$$

It was shown by Dehornoy (see [Dehornoy 1994] and [Dehornoy et al. 2002]) that each $B_{n}$ is right-orderable (but not orderable, for $n>2$ ). The positive cone consists of all elements expressible as a word in the $\sigma_{i}$ such that the generator with the lowest subscript occurs with only positive exponents. This right-order is discrete, with smallest positive element $\sigma_{n-1}$. On the other hand, it is shown in [Clay and Rolfsen 2007] that the Dehornoy order, when restricted to the commutator subgroup $B_{n}^{\prime}=\left[B_{n}, B_{n}\right]$, is a dense order for $n \geq 3$. For $n=3, B_{n}^{\prime}$ is free (on two generators). For $n \geq 5, B_{n}^{\prime}$ is finitely-generated and perfect [Gorin and Lin 1969], so $B_{n}$ is an example of a nonlocally indicable discretely right-orderable group for $n \geq 5$.

Now consider the braid group $B_{3}$ with its two generators $\sigma_{1}$ and $\sigma_{2}$ and let $H$ be the subgroup generated by $\sigma_{1}^{2}$ and $\sigma_{2}^{2}$. Crisp and Paris [2001] showed that $H$ is a free group with free basis $\sigma_{1}^{2}$ and $\sigma_{2}^{2}$. The Dehornoy order restricted to this 
subgroup has the least positive element $\sigma_{2}^{2}$. This gives an alternative construction of discrete right-orders on a free group.

Bergman [1991] published the first examples of groups which are right-orderable and not locally indicable; some of his examples are finitely generated and perfect. We shall argue that they can be given a discrete right-order.

If $G$ is a group acting on a set and $x_{1}, \ldots, x_{n}$ are elements of the set, then $\operatorname{Stab}_{G}\left(x_{1}, \ldots, x_{n}\right)$ will denote the pointwise stabilizer of $\left\{x_{1}, \ldots, x_{n}\right\}$ in $G$, namely $\left\{g \in G \mid g x_{i}=x_{i}\right.$ for all $\left.i\right\}$. Also $I$ will denote the identity matrix of $\mathrm{SL}_{2}(\mathbb{R})$. We have an action of $\mathrm{SL}_{2}(\mathbb{R})$ on the one point compactification $\overline{\mathbb{R}}=\mathbb{R} \cup\{\infty\} \cong S^{1}$, the circle, given by the rule

$$
\left(\begin{array}{ll}
a & b \\
c & d
\end{array}\right)(x)=\frac{a x+b}{c x+d}
$$

This induces a faithful action of $\mathrm{PSL}_{2}(\mathbb{R})$ on $\overline{\mathbb{R}}$. Let $H$ be a finitely generated subgroup of $\mathrm{SL}_{2}(\mathbb{R})$ containing the center $\{ \pm I\}$ of $\mathrm{SL}_{2}(\mathbb{R})$, and let $\bar{H}$ denote its image in $\mathrm{PSL}_{2}(\mathbb{R})$. Since $\mathbb{R}$ is the universal covering space of $S^{1}$, we can lift the action of $\bar{H}$ on $S^{1}$ to an action of a group $G$ on $\mathbb{R}$ by orientation preserving homeomorphisms. In this situation, $G$ will have a central subgroup $Z \cong \mathbb{Z}$ such that $G / Z \cong \bar{H}$ and $Z$ acts fixed point freely on $\mathbb{R}$. Also if $\pi: \mathbb{R} \rightarrow S^{1}$ is the associated covering map and $p \in \mathbb{R}$, then $\operatorname{Stab}_{\bar{H}}(\pi p)=Z \operatorname{Stab}_{G}(p) / Z \cong \operatorname{Stab}_{G}(p)$.

Proposition 4.6. Let $H$ be a finitely generated subgroup of $\mathrm{SL}_{2}(\mathbb{R})$ with $-I \in H$ and let $G$ be its lift to orientation preserving homeomorphisms of $\mathbb{R}$ (as described above). Suppose $H$ contains a diagonal matrix other than $\pm I$. Then $G$ has a discrete right order.

To prove this, we need an auxiliary result:

Lemma 4.7 [Linnell 2006, Lemma 2.2]. Let $G$ be a right ordered group, let $H$ be a convex subgroup of $G$ and let $<$ be any right order on $H$. Then there exists a right order on $G$ whose restriction to $H$ is $<$, and $H$ is still a convex subgroup under this new right order.

Proof of Proposition 4.6. Let us examine $\operatorname{Stab}_{H}(0)$ and $\operatorname{Stab}_{H}(0, \infty)$ with the action given by (4.5). The former is the lower triangular matrices, that is, the matrices above with $b=0$; we shall denote by $L$ those lower triangular matrices which lie in $H$. The latter is given by the diagonal matrices; we shall denote by $D$ those diagonal matrices which lie in $H$.

Thus we have an action of $H$ on $S^{1}$ and two points $p_{1}, p_{2} \in S^{1}$ such that $\operatorname{Stab}_{H}\left(p_{1}\right)=L$ and $\operatorname{Stab}_{H}\left(p_{1}, p_{2}\right)=D$. Let $p_{3} \in S^{1}$ be distinct from $p_{1}, p_{2}$. Then $\operatorname{Stab}_{H}\left(p_{1}, p_{2}, p_{3}\right)=\{ \pm I\}$. 
Now we can lift the action of $H$ on $S^{1}$ to an action of $G$ on $\mathbb{R}$ by orientation preserving homeomorphisms; $G$ will have a central subgroup $Z \cong \mathbb{Z}$ such that $G / Z \cong H /\{ \pm I\}$. For $i=1,2,3$, let $q_{i} \in \mathbb{R}$ be a lift of $p_{i}$. Then $Q:=$ $\operatorname{Stab}_{G}\left(q_{1}, q_{2}\right) \cong D /\{ \pm I\}$. We now define a right order on $G$ in the usual way when we have a group acting on $\mathbb{R}$. The positive cone is the set of all $g \in G$ such that $g\left(q_{i}\right)>q_{i}$ for the smallest $i$ such that $g\left(q_{i}\right) \neq q_{i}$. This right order will have the property that $Q$ is a (smallest nontrivial) convex subgroup of $G$. If $Q \cong \mathbb{Z}$, then it would follow that the above defined right order will be discrete, but this is not true in general. However since $H$ is finitely generated, $H \subseteq \mathrm{SL}_{2}(R)$ for some finitely generated subring $R$ of $\mathbb{R}$. By [Samuel 1966, Théorème 1], the group of units of a finitely generated integral domain is finitely generated, hence $D$ is also finitely generated. We deduce that $D /\{ \pm I\}$ is a finitely generated free abelian group. Thus $Q$ is also a finitely generated free abelian group and hence has a discrete right order by Corollary 2.2. The result now follows from Lemma 4.7 .

\section{References}

[Baumslag 1968] G. Baumslag, "On the residual nilpotence of certain one-relator groups", Comm. Pure Appl. Math. 21 (1968), 491-506. MR 38 \#3327 Zbl 0186.32101

[Bergman 1991] G. M. Bergman, "Right orderable groups that are not locally indicable", Pacific J. Math. 147:2 (1991), 243-248. MR 92e:20030 Zbl 0677.06007

[Botto Mura and Rhemtulla 1977] R. Botto Mura and A. Rhemtulla, Orderable groups, Lecture Notes in Pure and Applied Mathematics 27, Marcel Dekker, New York, 1977. MR 58 \#10652 Zbl 1071.20508

[Brodskiı̌ 1984] S. D. Brodskiŭ, "Equations over groups, and groups with one defining relation", Sibirsk. Mat. Zh. 25:2 (1984), 84-103. MR 86e:20026 Zbl 0579.20020

[Burns and Hale 1972] R. G. Burns and V. W. D. Hale, "A note on group rings of certain torsion-free groups”, Canad. Math. Bull. 15 (1972), 441-445. MR 46 \#9149 Zbl 0244.16006

[Charney 2007] R. Charney, “An introduction to right-angled Artin groups”, Geom. Dedicata 125 (2007), 141-158. MR 2008f:20076 Zbl 1152.20031

[Clay and Rolfsen 2007] A. Clay and D. Rolfsen, "Densely ordered braid subgroups", J. Knot Theory Ramifications 16:7 (2007), 869-877. MR 2008g:20077 Zbl 1139.20033

[Crisp and Paris 2001] J. Crisp and L. Paris, "The solution to a conjecture of Tits on the subgroup generated by the squares of the generators of an Artin group", Invent. Math. 145:1 (2001), 19-36. MR 2002j:20069 Zbl 1002.20021

[Dehornoy 1994] P. Dehornoy, "Braid groups and left distributive operations", Trans. Amer. Math. Soc. 345:1 (1994), 115-150. MR 95a:08003 Zbl 0837.20048

[Dehornoy et al. 2002] P. Dehornoy, I. Dynnikov, D. Rolfsen, and B. Wiest, Why are braids orderable?, Panoramas et Synthèses [Panoramas and Syntheses] 14, Société Mathématique de France, Paris, 2002. MR 2004e:20062 Zbl 1048.20021

[Duchamp and Krob 1992] G. Duchamp and D. Krob, "The lower central series of the free partially commutative group”, Semigroup Forum 45:3 (1992), 385-394. MR 93e:20047 Zbl 0814.20025 
[Gorin and Lin 1969] E. A. Gorin and V. J. Lin, "Algebraic equations with continuous coefficients and some problems of the algebraic theory of braids", Mat. Sb. (N.S.) 78 (1969), 579-610. In Russian; translated in Math. USSR, Sb. 7 (1969), 569-596. MR 40 \#4939 Zbl 0211.54905

[Linnell 2001] P. A. Linnell, "Left ordered groups with no non-abelian free subgroups", J. Group Theory 4:2 (2001), 153-168. MR 2002h:06018 Zbl 0982.06013

[Linnell 2006] P. A. Linnell, "The topology on the space of left orderings of a group", preprint, 2006, Available at http://arxiv.org/abs/math/0607470. arXiv math/0607470v2

[Longobardi et al. 2000] P. Longobardi, M. Maj, and A. Rhemtulla, "When is a right orderable group locally indicable?", Proc. Amer. Math. Soc. 128 (2000), 637-641. MR 2000j:20072 Zbl 0941.20037

[Morris 2006] D. W. Morris, "Amenable groups that act on the line", Algebr. Geom. Topol. 6 (2006), 2509-2518. MR 2008c:20078 Zbl pre05118602

[Samuel 1966] P. Samuel, “À propos du théorème des unités”, Bull. Sci. Math. (2) 90 (1966), 89-96. MR 34 \#4296 Zbl 0166.30701

Communicated by Efim Zelmanov

Received 2008-08-19 Revised 2009-03-09 Accepted 2009-04-17

linnell@math.vt.edu Department of Mathematics, Virginia Tech, Blacksburg, VA 24061-0123, United States http://www.math.vt.edu/people/plinnell/

akbar@math.ualberta.ca

Department of Mathematical \& Statistical Sciences, University of Alberta, Edmonton, AL T6G2G1, Canada http://www.math.ualberta.ca/Rhemtulla_A.html

rolfsen@math.ubc.ca

University of British Columbia, Mathematics Department, 1984 Mathematics Road, Vancouver, BC V6T 1Z2, Canada http://www.math.ubc.ca/ rolfsen/ 


\title{
The half-twist for $U_{q}(\mathfrak{g})$ representations
}

\author{
Noah Snyder and Peter Tingley
}

\begin{abstract}
We introduce the notion of a half-ribbon Hopf algebra, which is a Hopf algebra $\mathcal{H}$ along with a distinguished element $t \in \mathcal{H}$ such that $(\mathcal{H}, R, C)$ is a ribbon Hopf algebra, where $R=\left(t^{-1} \otimes t^{-1}\right) \Delta(t)$ and $C=t^{-2}$. The element $t$ is closely related to the topological "half-twist", which twists a ribbon by 180 degrees. We construct a functor from a topological category of ribbons with half-twists to the category of representations of any half-ribbon Hopf algebra. We show that $U_{q}(\mathfrak{g})$ is a (topological) half-ribbon Hopf algebra, but that $t^{-2}$ is not the standard ribbon element. For $U_{q}\left(\mathfrak{s l}_{2}\right)$, we show that there is no half-ribbon element $t$ such that $t^{-2}$ is the standard ribbon element. We then discuss how ribbon elements can be modified, and some consequences of these modifications.
\end{abstract}

1. Introduction 809

2. Conventions 811

3. Background 812

4. Half-ribbon Hopf algebras 816

5. Ribbon and half-ribbon elements for $U_{q}(\mathfrak{g}) \quad 824$

6. Questions 832

Acknowledgments $\quad 833$

References $\quad 833$

\section{Introduction}

Let $\mathcal{R} \mathcal{I B B O N}(S)$ be the category whose morphisms consist of tangles of oriented directed ribbons up to isotopy, each labeled with an element of some set $S$. There is a notion of a ribbon Hopf algebra $\mathcal{H}$ (see [Chari and Pressley 1994], for example), which is related to this topological category by the fact that there is a monoidal functor $\mathcal{F}^{\prime}$ from $\mathcal{R} \mathcal{I B B O N}(\mathcal{H}$-rep) to the category of representations $\mathcal{H}$-rep. This allows one to construct invariants of oriented framed links, and from there invariants of ordinary links.

MSC2000: primary 17B37; secondary 57T05, 57M05.

Keywords: quantum group, Hopf algebra, ribbon category.

Both authors were supported in part by RTG grant DMS-0354321. Tingley was also partly supported by the Australian Research Council grant DP0879951 and NSF grant DMS-0902649. 
There is a morphism in $\mathcal{R} \mathcal{I B B O N}(S)$ which twists a ribbon by 360 degrees, but not one that twists a ribbon by 180 degrees (since negatively oriented objects are not allowed). We propose that one should consider a slightly larger category, denoted $\mathcal{H} \mathcal{R} \mathcal{I B}(S)$, where this 180 degree twist, the "half-twist," is allowed. The half-twist can be applied to several ribbons at once, and all morphisms in $\mathcal{H} \mathcal{R} \mathcal{I B}(S)$ can be constructed out of half-twists, along with various caps and cups. The following isotopy shows how the crossing in $\mathcal{H} \mathcal{R} \mathcal{I B}(S)$ is constructed out of the half-twist:

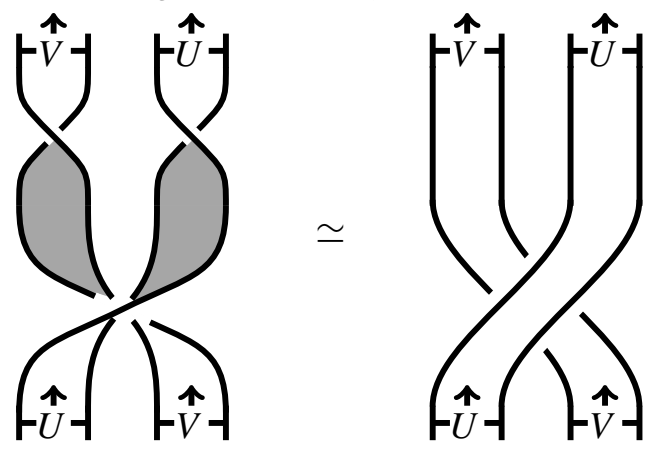

Recall that a ribbon Hopf algebra $\mathcal{H}$ is a Hopf algebra along with two extra features:

- A universal $R$-matrix $R \in \mathcal{H} \otimes \mathcal{H}$. The functor $\mathcal{F}^{\prime}$ takes a simple crossing of ribbons labeled $V$ and $W$ to Flip $\circ R$ acting on $V \otimes W$.

- A central "ribbon" element $C \in \mathcal{H}$. The functor $\mathcal{F}^{\prime}$ takes a 360 degree twist of a ribbon labeled $V$ to $C$ acting on $V$.

The elements $R$ and $C$ must satisfy various compatibility conditions (see Section 4A).

In the present work we define a half-ribbon Hopf algebra to be a ribbon Hopf algebra along with a distinguished element $t$ such that

(i) $R=\left(t^{-1} \otimes t^{-1}\right) \Delta(t)$,

(ii) $C=t^{-2}$.

We show that, if $\mathcal{H}$ is a half-ribbon Hopf algebra, then $\mathcal{F}^{\prime}$ can be extended to a functor $\mathcal{F}$ from $\mathcal{H} \mathcal{R} \mathcal{I B}(S)$ to $\mathcal{H}$-rep.

Our main interest is the case where $\mathcal{H}$ is the quantized universal enveloping algebra $U_{q}(\mathfrak{g})$ of a finite-dimensional complex simple Lie algebra $\mathfrak{g}$. In this case $U_{q}(\mathfrak{g})$ is actually a topological ribbon Hopf algebra, meaning that $R$ and $C$ only lie in some completion, not in $U_{q}(\mathfrak{g})$ itself. We define a topological half-ribbon Hopf algebra by allowing $t$ to lie in a completion of $\mathcal{H}$, and show that $U_{q}(\mathfrak{g})$ has this structure. The main ingredient is a formula for the $R$ matrix of $U_{q}(\mathfrak{g})$, due to Kirilov and Reshetikhin [1990] and Levendorskil and Soibelman [1991], of the form

$$
R=\left(X^{-1} \otimes X^{-1}\right) \Delta(X) .
$$


The correspondence to condition (i) above should be clear, and we show that $X$ is in fact a half-ribbon element for $U_{q}(\mathfrak{g})$. Interestingly, $X^{-2}$ is not the standard ribbon element, and in fact we show that in certain cases it is not possible to find a half-ribbon element $t$ for $U_{q}(\mathfrak{g})$ such that $t^{-2}$ is the standard ribbon element.

In Section 5 we discuss the different ribbon elements for $U_{q}(\mathfrak{g})$, and in some small cases we describe exactly which ones arise from half-ribbon elements. We then discuss some consequences of using $X^{-2}$ in place of the usual ribbon element. In particular, it simplifies the correspondence between certain skein theoretic constructions of link invariants and the quantum group constructions of the same objects, essentially by explaining some annoying negative signs that appear in, for example, [Ohtsuki 2002] or [Kuperberg 1996].

We feel it would be of considerable interest to study which ribbon Hopf algebras can be given the structure of half-ribbon Hopf algebras. It would also be nice to give straightforward conditions on an element $t$ in a general Hopf algebra $\mathcal{H}$, such that $\mathcal{H}$ along with $t$ is a half-ribbon Hopf algebra (that is, $\mathcal{H}$ is a ribbon Hopf algebra, with $R=\left(t^{-1} \otimes t^{-1}\right) \Delta(t)$ and $\left.C=t^{-2}\right)$. At the end of the paper we discuss these and other possible future directions.

\section{Conventions}

We first fix some notation. For the most part we follow conventions from [Chari and Pressley 1994].

- $\mathfrak{g}$ is a complex simple Lie algebra with Cartan algebra $\mathfrak{h}$, and $A=\left(a_{i j}\right)_{i, j \in I}$ is its Cartan matrix.

- $\langle\cdot, \cdot\rangle$ denotes the paring between $\mathfrak{h}$ and $\mathfrak{h}^{\star}$ and $(\cdot, \cdot)$ denotes the usual symmetric bilinear form on either $\mathfrak{h}$ or $\mathfrak{h}^{\star}$. Fix the usual bases $\alpha_{i}$ for $\mathfrak{h}^{\star}$ and $H_{i}$ for $\mathfrak{h}$, and recall that $\left\langle H_{i}, \alpha_{j}\right\rangle=a_{i j}$.

- $d_{i}=\left(\alpha_{i}, \alpha_{i}\right) / 2$, so that $\left(H_{i}, H_{j}\right)=d_{j}^{-1} a_{i j}$. Let $B$ denote the matrix $\left(d_{j}^{-1} a_{i j}\right)$.

- $q_{i}=q^{d_{i}}$.

- $\rho$ is the element of $\mathfrak{h}^{*}$ such that $\left(\alpha_{i}, \rho\right)=d_{i}$ for all $i$.

- $\rho^{\vee}$ is the element of $\mathfrak{h}$ such that $\left\langle\alpha_{i}, \rho^{\vee}\right\rangle=1$ for all $i$.

- $s_{i}$ is the element of the Weyl group which is defined by

$$
s_{i}\left(\alpha_{j}\right)=\alpha_{j}-\left\langle\alpha_{i}, \alpha_{j}^{\vee}\right\rangle \alpha_{i} .
$$

- $\theta$ is the diagram automorphism such that $w_{0}\left(\alpha_{i}\right)=-\alpha_{\theta(i)}$, where $w_{0}$ is the longest element in the Weyl group.

- $U_{q}(\mathfrak{g})$ is the quantized universal enveloping algebra associated to $\mathfrak{g}$, generated over $\mathbb{C}(q)$ by $E_{i}, F_{i}$ for all $i \in I$, and $K_{w}$ for $w$ in the coweight lattice of $\mathfrak{g}$. As usual, let $K_{i}=K_{H_{i}}$. We actually must adjoin a fixed $k$ th root of $q$ to the base field, for some $k$ depending on $\mathfrak{g}$. This causes no real difficulty, and for the most part 
we ignore it. For convenience, we recall the exact formula for the coproduct and antipode:

$$
\left\{\begin{array} { l } 
{ \Delta E _ { i } = E _ { i } \otimes K _ { i } + 1 \otimes E _ { i } , } \\
{ \Delta F _ { i } = F _ { i } \otimes 1 + K _ { i } ^ { - 1 } \otimes F _ { i } , } \\
{ \Delta K _ { i } = K _ { i } \otimes K _ { i } , }
\end{array} \quad \left\{\begin{array}{l}
S\left(E_{i}\right)=-E_{i} K_{i}^{-1}, \\
S\left(F_{i}\right)=-K_{i} F_{i}, \\
S\left(K_{i}\right)=K_{i}^{-1}
\end{array}\right.\right.
$$

- $[n]=\left(q^{n}-q^{-n}\right) /\left(q-q^{-1}\right)$, and $X^{(n)}=\left(X^{n}\right) /([n][n-1] \cdots[2])$.

- $V_{\lambda}$ is the irreducible representation of $U_{q}(\mathfrak{g})$ with highest weight $\lambda$, and $v_{\lambda}$ is a highest weight vector.

- $P$ is the weight lattice for $\mathfrak{g}$ and $Q$ is the root lattice.

- The standard ribbon element $C$ for $U_{q}(\mathfrak{g})$ acts on $V_{\lambda}$ as the constant

$$
q^{-(\lambda, \lambda)-2(\lambda, \rho)} \text {. }
$$

- $U_{q}(\mathfrak{g})$-rep is the category of finite-dimensional Type 1 representations.

- $(\mathcal{H}, R)$ is a quasitriangular Hopf algebra over a field $F$, where

(i) $\mu: \mathcal{H} \otimes \mathcal{H} \rightarrow \mathcal{H}$ is multiplication;

(ii) $l: F \rightarrow \mathcal{H}$ is the unit;

(iii) $\Delta: \mathcal{H} \rightarrow \mathcal{H} \otimes \mathcal{H}$ is the comultiplication;

(iv) $\varepsilon: \mathcal{H} \rightarrow F$ is the counit;

(v) $S: \mathcal{H} \rightarrow \mathcal{H}$ is the antipode.

- Flip : $V \otimes W \rightarrow W \otimes V$ is defined by Flip $(v \otimes w)=w \otimes v$. For longer tensor products, define rev $: V_{1} \otimes \cdots \otimes V_{k} \rightarrow V_{k} \otimes \cdots V_{1}$ by $v_{1} \otimes \cdots \otimes v_{k} \mapsto v_{k} \otimes \cdots \otimes v_{1}$.

- If $V$ is a representation of a Hopf algebra $H$, then we define the left dual $V^{*}$ to be the dual vector space with the action $x \circ f(v)=f(S(x) v)$, and the right dual ${ }^{*} V$ to be the dual vector space with the action $x \circ f(v)=f\left(S^{-1}(x) v\right)$. Note that $\left({ }^{*} V\right)^{*}=V={ }^{*}\left(V^{*}\right)$.

\section{Background}

Much of the motivation for this paper comes from studying an expression for the $R$-matrix

$$
R=\left(X^{-1} \otimes X^{-1}\right) \Delta(X),
$$

where $X$ is an element in some completion of $U_{q}(\mathfrak{g})$. This was first introduced by Kirilov and Reshetikhin [1990] and Levendorskil and Soibelman [1991], and has recently proven useful in studying the relationship between the braiding and crystal bases [Kamnitzer and Tingley 2009]. In this section we review this formula, and also recall the definition and basic properties of a ribbon Hopf algebra. 
3A. A completion of $\boldsymbol{U}_{\boldsymbol{q}}(\mathfrak{g})$. The element $X$ from (5) is not actually in $U_{q}(\mathfrak{g})$, but only in a completion. In order to be precise, we briefly review the completion of $U_{q}(\mathfrak{g})$ that we use.

Definition 3.1. $\widetilde{U_{q}(\mathfrak{g})}$ is the completion of $U_{q}(\mathfrak{g})$ in the weak topology generated by all matrix elements of all (finite-dimensional Type 1) representations. Similarly, $U_{q}(\mathfrak{g}) \otimes U_{q}(\mathfrak{g})$ is the completion of $U_{q}(\mathfrak{g}) \otimes U_{q}(\mathfrak{g})$ in the weak topology defined by all matrix elements of representations $V_{\lambda} \otimes V_{\mu}$, for all ordered pairs $(\lambda, \mu)$.

Theorem 3.2 [Kamnitzer and Tingley 2009]. $\widetilde{U_{q}(\mathfrak{g})}$ is the direct product of the endomorphism rings of all irreducible representations of $U_{q}(\mathfrak{g})$. That is,

$$
\widetilde{U_{q}(\mathfrak{g})}=\prod_{\lambda \in \Lambda_{+}} \operatorname{End}\left(V_{\lambda}\right)
$$

Comment 3.3. It is straightforward to see that $\widetilde{U_{q}(\mathfrak{g})}$ is a topological Hopf algebra.

By Theorem 3.2, specifying an element of $\widetilde{U_{q}(\mathfrak{g})}$ is exactly the same as specifying how it acts on each isomorphism class of irreducible representation. Similarly, if we want to specify an element of $\widetilde{U_{q}(\mathfrak{g})^{\otimes 2}}$ we just need to say how it acts on every tensor product of any two irreducible representations.

3B. A method of constructing commutativity constraints. The next result is a development of an idea introduced in [Henriques and Kamnitzer 2006] to study the crystal commutor; the proof is a straightforward exercise.

Proposition 3.4 [Kamnitzer and Tingley 2009, Proposition 3.11]. Let $Y$ be an invertible element in $\widetilde{U_{q}(\mathfrak{g})}$ such that the map $C_{Y}: X \rightarrow Y X Y^{-1}$ restricts to an algebra automorphism of $U_{q}(\mathfrak{g})$. Then $C_{Y}$ is a coalgebra antiautomorphism if and only if, for every pair of representations $V$ and $W$, the map

$$
\begin{aligned}
\sigma_{V, W}^{Y}: V \otimes W & \rightarrow W \otimes V \\
v \otimes w & \rightarrow \text { Flip } \circ\left(Y^{-1} \otimes Y^{-1}\right) \Delta(Y) v \otimes w
\end{aligned}
$$

is an isomorphism.

Given an element $Y$ satisfying the conditions of Proposition 3.4, we use the notation $\sigma^{Y}$ to denote the system of isomorphisms $\left\{\sigma_{V, W}^{Y}\right\}$.

3C. The element $\boldsymbol{X}$. We now explicitly describe the element $X$ from (5). We will need a way to specify a lowest weight vector of $V_{\lambda}$, depending linearly on a chosen of highest weight vector. We do this using the action of the braid group on $V_{\lambda}$. We very briefly review this theory, and refer the reader to, for example, [Chari and Pressley 1994, Chapter 8.1.2] or [Lusztig 1993] for more details. 
Definition 3.5 [Lusztig 1993, 5.2.1]. $T_{i}$ is the element of $\widetilde{U_{q}(\mathfrak{g})}$ that acts on a weight vector $v$ by:

$$
T_{i}(v)=\sum_{\substack{a, b, c \geq 0 \\ a-b+c=\left(\operatorname{wt}(v), \alpha_{i}\right)}}(-1)^{b} q_{i}^{a c-b} E_{i}^{(a)} F_{i}^{(b)} E_{i}^{(c)} v .
$$

By [Lusztig 1993, Theorem 39.4.3], these $T_{i}$ generate an action of the braid

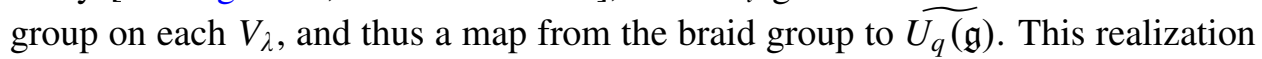
of the braid group is often referred to as the quantum Weyl group. It is related to the classical Weyl group by the fact that, for any weight vector $v \in V, \operatorname{wt}\left(T_{i}(v)\right)=$ $s_{i}(\mathrm{wt}(v))$.

Definition 3.6. Let $w_{0}=s_{i_{1}} s_{i_{2}} \cdots s_{i_{N}}$ be a reduced expression for the longest word $w_{0}$ in the Weyl group. Then $T_{w_{0}}=T_{i_{1}} T_{i_{2}} \cdots T_{i_{N}}$.

It is clear that $T_{w_{0}}$ is well defined because the elements $T_{i}$ satisfy the braid relations. Note that $T_{w_{0}}$ interchanges the highest and lowest weight spaces of $V_{\lambda}$.

Definition 3.7. $v_{\lambda}^{\text {low }}$ is the element in the lowest weight space of $V_{\lambda}$ defined by

$$
T_{w_{0}}\left(v_{\lambda}^{\text {low }}\right)=v_{\lambda}
$$

where $v_{\lambda}$ is the chosen highest weight vector.

Definition 3.8. $J$ is the element of $\widetilde{U_{q}(\mathfrak{g})}$ defined by, for any weight vector $v \in V_{\lambda}$,

$$
J(v)=q^{(\mathrm{wt}(v), \operatorname{wt}(v)) / 2+(\operatorname{wt}(v), \rho)} v .
$$

Definition 3.9. $X=J T_{w_{0}}$.

Lemma 3.10 [Kamnitzer and Tingley 2009, Section 5.2]. The element $X$ has the following properties:

(i) $X\left(v_{\lambda}^{\text {low }}\right)=q^{(\lambda, \lambda) / 2+(\lambda, \rho)} v_{\lambda}$.

(ii) $X\left(v_{\lambda}\right)=(-1)^{\left\langle 2 \lambda, \rho^{\vee}\right\rangle} q^{(\lambda, \lambda) / 2+(\lambda, \rho)} v_{\lambda}^{\text {low }}$.

(iii) $X^{2}$ is central, and acts on $V_{\lambda}$ as multiplication by the scalar

$$
(-1)^{\left\langle 2 \lambda, \rho^{\vee}\right\rangle} q^{(\lambda, \lambda)+2(\lambda, \rho)} \text {. }
$$

(iv) $C_{X}$ is given by

$$
\left\{\begin{array}{l}
C_{X}\left(E_{i}\right)=-F_{\theta(i)} \\
C_{X}\left(F_{i}\right)=-E_{\theta(i)}, \\
C_{X}\left(K_{i}\right)=K_{\theta(i)}^{-1}
\end{array}\right.
$$

We next present a key result: 
Theorem 3.11 ([Kirillov and Reshetikhin 1990, Theorem 3; Levendorskiŭ and Soĭbel'man 1991, Theorem 1]; see [Kamnitzer and Tingley 2009] for this exact statement.). $\sigma^{X}$ is the standard braiding. Equivalently, the standard $R$-matrix for $U_{q}(\mathfrak{g})$ can be realized as

$$
R=\left(X^{-1} \otimes X^{-1}\right) \Delta(X) .
$$

In fact, we will take (9) as the definition of the universal $R$-matrix.

\section{D. Ribbon Hopf algebras.}

Definition 3.12 [Chari and Pressley 1994, Definition 4.2.8]. A ribbon Hopf algebra $(\mathcal{H}, R, v)$ is a quasitriangular Hopf algebra $(\mathcal{H}, R)$ equipped with an invertible central element $v$ such that

(i) $v^{2}=u S(u)$, where $u=\mu(S \otimes i d) R_{21}$,

(ii) $S(v)=v$,

(iii) $\varepsilon(v)=1$,

(iv) $\Delta(v)=(v \otimes v)\left(R_{21} R_{12}\right)^{-1}$.

Proposition 3.13 [Chari and Pressley 1994]. The element $g:=v^{-1} u$ is grouplike (where $u$ is as in Definition 3.12(i)).

The following four maps are crucial to studying ribbon Hopf algebras:

Definition 3.14 [Chari and Pressley 1994, page 163]. Let $(\mathcal{H}, R, v)$ be a ribbon Hopf algebra, and $(V, \pi)$ a representation of $\mathcal{H}$. Let $f \in V^{*}$ and $v \in V$. Let $e_{i}$ and $e^{i}$ be dual bases of $V$ and $V^{*}$ respectively. Then

(i) $\operatorname{ev}(f \otimes v)=f(v)$;

(ii) $\tilde{\mathrm{ev}}(v \otimes f)=f(g v)$;

(iii) $\operatorname{coev}(1)=\sum e_{i} \otimes e^{i}$;

(iv) $\widetilde{\operatorname{coev}}(1)=\sum e^{i} \otimes g^{-1} e_{i}$.

Recall that $V \otimes V^{*}$ can be identified with $\operatorname{End}(V)$. Under this identification $\widetilde{\mathrm{eV}}$ is the quantum trace of [Reshetikhin and Turaev 1991].

We will be working with $U_{q}(\mathfrak{g})$, which is not a ribbon Hopf algebra according to the above definition, since the elements $v$ and $R$ actually lie in the completions $\widetilde{U_{q}(\mathfrak{g})}$ and $U_{q}\left(\widetilde{\mathfrak{g}) \otimes U_{q}}(\mathfrak{g})\right.$ discussed in Section 3A. This is known as a topological ribbon Hopf algebra. The theory goes through just as well in the topological case.

Definition 3.15. Let $C$ be the element of $\widetilde{U_{q}(\mathfrak{g})}$ which acts on the representation $V_{\lambda}$ as multiplication by $q^{-(\lambda, \lambda)-2(\lambda, \rho)}$.

The following is well known (see [Chari and Pressley 1994, Corollary 8.3.16], for example).

Theorem 3.16. $\left(U_{q}(\mathfrak{g}), R, C\right)$ is a topological ribbon Hopf algebra. 
3E. The Temperley-Lieb category. The unoriented Temperley-Lieb category $T L$ has as objects collections of points on the real line up to isotopy, and a morphism from $A$ to $B$ is a formal linear combination of "planar arc diagrams" each of which is a collection of nonintersecting segments in $\mathbb{R} \times[0,1]$ whose boundary consists of $A \times 0$ and $B \times 1$ modulo isotopy in the plane and the following relation:

$$
\bigcirc-q-q^{-1} \text {. }
$$

As discussed in, for example, [Goodman and Wenzl 2002], TL is a rigid monoidal category, where composition is given by vertical stacking, tensor product is given by disjoint union, and the dual is given by 180-degree rotation. Since the double dual acts trivially, this is a pivotal category with the trivial pivotal structure.

\section{Half-ribbon Hopf algebras}

This section contains the definition of a half-ribbon Hopf algebra. It also explains the relationship with the topological category $\mathcal{H} \mathcal{R} \mathcal{I B}(S)$.

\section{A. Definition and basic properties.}

Definition 4.1. A half-ribbon Hopf algebra is a Hopf algebra, together with a distinguished element $t \in \mathcal{H}$, such that $(\mathcal{H}, R, v)$ is a ribbon Hopf algebra, where $R=\left(t^{-1} \otimes t^{-1}\right) \Delta(t)$ and $v=t^{-2}$.

In the case where $t$ only exists in a completion of $\mathcal{H}$, we say $\mathcal{H}$ is a topological half-ribbon Hopf algebra.

Proposition 4.2. A half-ribbon element $t$ in a half-ribbon Hopf algebra has the following properties:

(i) The algebra automorphism $C_{t}: \mathcal{H} \rightarrow \mathcal{H}$ defined by $x \rightarrow t x t^{-1}$ is also a coalgebra antiautomorphism.

(ii) $\varepsilon(t)=1$. Equivalently, $t$ acts as the identity on the trivial representation.

(iii) $S(t)^{2}=t^{2}$.

Proof. (i) follows from $R \Delta(x)=\Delta^{o p}(x) R$.

(ii) follows from the fact that $R$ acts as the identity on $\mathbf{1} \otimes V$, where $\mathbf{1}$ is the trivial representation.

(iii) follows because $t^{-2}$ is a ribbon element so $S\left(t^{-2}\right)=t^{-2}$.

Proposition 4.3. The various important elements in a ribbon Hopf algebra can be written in terms of a half-ribbon elements $t$ as follows:

$$
\begin{array}{c|c|c|c}
R & v & u & g \\
\hline\left(t^{-1} \otimes t^{-1}\right) \Delta(t)=\Delta^{o p}(t)\left(t^{-1} \otimes t^{-1}\right) & t^{-2} & S\left(t^{-1}\right) t^{-1} & S(t) t^{-1}=S\left(t^{-1}\right) t
\end{array}
$$


Proof. $(R)$ : The first formula is part of the definition of a half-ribbon element. The second formula follows by Proposition 4.2(i) since this implies that, for all $x \in \mathcal{H}$,

$$
\left(t^{-1} \otimes t^{-1}\right) \Delta(x)(t \otimes t)=\Delta^{o p}\left(t^{-1} x t\right) .
$$

$(v)$ : This is part of the definition of a half-ribbon element.

(u): By definition, $u=\mu \circ(S \otimes 1) R_{21}$, where $\mu$ is multiplication. Thus:

$$
\begin{aligned}
u & =\mu \circ(S \otimes 1)\left(\left[\left(\Delta^{o p}(t)\left(t^{-1} \otimes t^{-1}\right)\right]_{21}\right)\right. \\
& =\mu\left[\left((S \otimes 1)(\Delta(t))\left(t^{-1} \otimes t^{-1}\right)\right]\right. \\
& =\sum S\left(t^{-1}\right) S\left(t_{1}\right) t_{2} t^{-1} \\
& =S\left(t^{-1}\right)[\imath \circ \varepsilon(t)] t^{-1} \\
& =S\left(t^{-1}\right) t^{-1} .
\end{aligned}
$$

In (14) we have used Sweedler's notation $\Delta(x)=\sum x_{1} \otimes x_{2}$. The equality (15) follows from the antipode axiom of a Hopf algebra, and (16) follows by Proposition 4.2(ii).

( $g$ ): By definition, $g=v^{-1} u$. Recall that $v$ is central. To get the second equation, apply Proposition 4.2(iii).

Comment 4.4. For historical reasons the ribbon element $v$ represents a negative twist. We use $t$ for the positive half-twist, which explains the fact that $v=t^{-2}$.

4B. A topological category of ribbons with half-twists. There is a functor from a certain topological category to the category of representations of any ribbon Hopf algebra, which allows one to construct topological invariants. For a half-ribbon Hopf algebra, this functor can be extended to a larger topological category. In this section we define the two relevant categories, beginning with the large one.

Definition 4.5. The category $\mathcal{H} \mathcal{R} \mathcal{I B}(S)$ of topological half-ribbons with labels in some set $S$ is defined by:

- Objects in $\mathcal{H} \mathcal{R} \mathcal{I B}(S)$ consist of a finite number of disjoint closed intervals on the real line, each labeled with an element of $S$, each with a choice of shading (shaded or unshaded), and each directed (up or down). These objects are considered up to isotopy of the real line. For example:

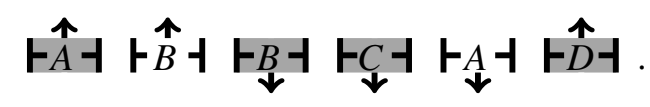

- A morphism between two objects $A, B \in \mathcal{H} \mathcal{R} \mathcal{I B}(S)$ consist of a "tangle of orientable, directed ribbons" in $\mathbb{R}^{2} \times I$, whose loose ends are exactly

$$
(A, 0,0) \cup(B, 0,1) \subset \mathbb{R} \times \mathbb{R} \times I,
$$


along with a choice of direction and shading of each ribbon. These must be such that the direction (up or down) of each interval in $A \cup B$ agrees with the direction of the ribbon whose end lies at that interval, and the shading (light or dark) of each interval agrees with the shading on the visible side of the ribbon near that interval. These are considered up to isotopy.

- Composition of two morphisms is given by stacking them on top of each other, and them shrinking the vertical axis by a factor of two. Note that we read our diagrams bottom to top. For example:

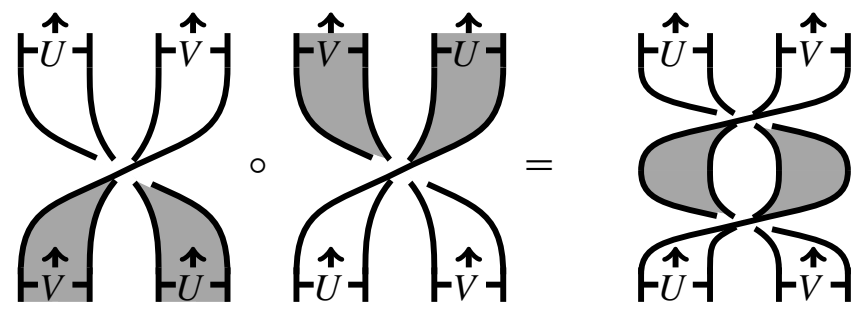

Definition 4.6. $\mathcal{R} \mathcal{I B B O N}(S)$ is the full subcategory of $\mathcal{H} \mathcal{R} \mathcal{I B}(S)$ consisting of those objects all of whose intervals are unshaded.

The definition of $\mathcal{H} \mathcal{R} \mathcal{I B}(S)$ can easily be made rigorous, in the same way as is done for the subcategory $\mathcal{R} \mathcal{I B B O N}(S)$; see for example [Chari and Pressley 1994]. If fact, as with $\mathcal{R} \mathcal{I B B O N}(S), \mathcal{H} \mathcal{R} \mathcal{I B}(S)$ is a rigid monoidal category, where tensor products and duals are shown below:

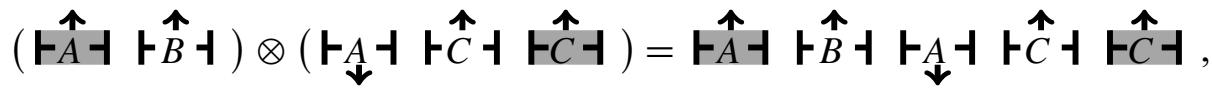

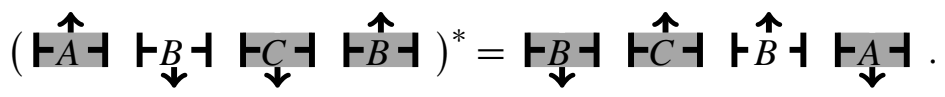

4C. A functor from $\mathcal{H} \mathcal{R} \mathcal{I B}(S)$ to $\mathcal{H}$-rep. We will now show that the category of representations of a half-ribbon Hopf algebra admits a natural functor from $\mathcal{H} \mathcal{R} \mathcal{I}(\mathcal{H}$-rep). This theorem is an extension of the corresponding result relating ribbon Hopf algebras and $\mathcal{R} \mathcal{I B B O} \mathcal{N}(\mathcal{H}$-rep), so we begin by stating this known result.

Theorem 4.7 (see [Reshetikhin 1989], [Shum 1994], or [Chari and Pressley 1994, Theorem 5.3.2]). Let $(\mathcal{H}, R, C)$ be a ribbon Hopf algebra. There is a unique monoidal functor $\mathcal{F}^{\prime}$ from $\mathcal{R} \mathcal{I B B O} \mathcal{N}(\mathcal{H}$-rep) to $\mathcal{H}$-rep satisfying the following conditions:

$$
\mathcal{F}^{\prime}(\stackrel{\mathfrak{r} V}{V})=V \quad \text { and } \quad \mathcal{F}^{\prime}\left(\boldsymbol{H}_{\Downarrow} \dashv\right)=V^{*}
$$


(ii)
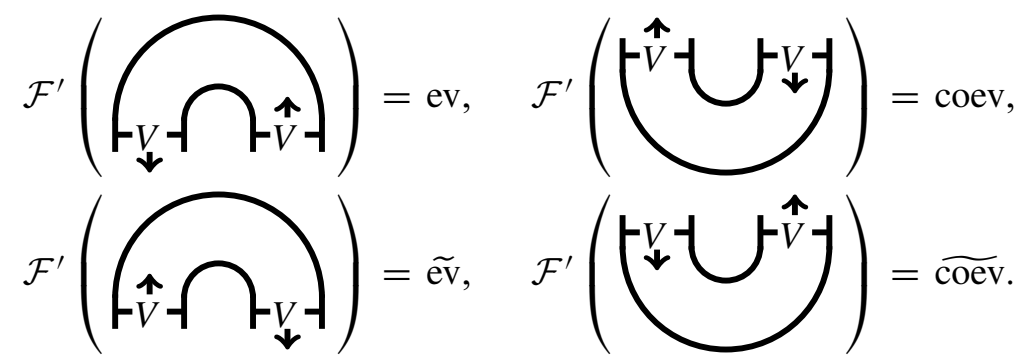

(iii)

$$
\mathcal{F}^{\prime}\left(\zeta^{\prime}\right)=C,
$$

thought of as a morphism from $V$ to $V$ or from $V^{*}$ to $V^{*}$, depending on the orientation.

$$
\left.\mathcal{F}^{\prime}(Y / /)\right)=\text { Flip } \circ R
$$

as a morphism from the tensor product of the bottom two objects to the tensor product of the top two objects, regardless of labeling and orientation.

Comment 4.8. Warning: this functor is not a strict rigid functor. Notice that

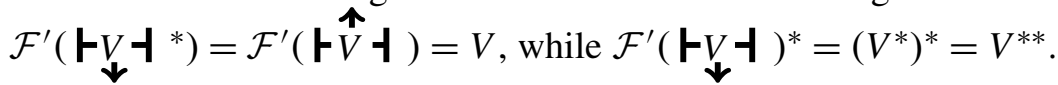

Our main result for this section is that $\mathcal{F}^{\prime}$ can be extended to a functor $\mathcal{F}$ : $\mathcal{H} \mathcal{R} \mathcal{I}(\mathcal{H}$-rep $) \rightarrow \mathcal{H}$-rep as follows.

Definition 4.9. Let $V$ be a representation of a half-ribbon Hopf algebra $\mathcal{H}$ and for each $x \in \mathcal{H}$ let $\pi_{V}(x)$ be the element of $\operatorname{End}(V)$ defined by $x$. Define a new representation $V^{C_{t}}$ which is equal to $V$ as a vector space, but with the action of $x \in \mathcal{H}$ defined by $\pi_{V C_{t}}(x)=\pi_{V}\left(t x t^{-1}\right)$.

Definition 4.10. The topological half-twist is the morphism $I_{n}$ in $\mathcal{H} \mathcal{R} \mathcal{I B}$ which takes $n$ ribbons of any shading and orientation, and twists them all together by 180 degrees:

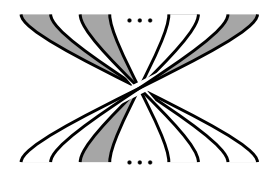

Theorem 4.11. There is a unique monoidal functor $\mathcal{F}$ from $\mathcal{H} \mathcal{R} \mathcal{I B}(\mathcal{H}$-rep) to $\mathcal{H}$-rep satisfying:

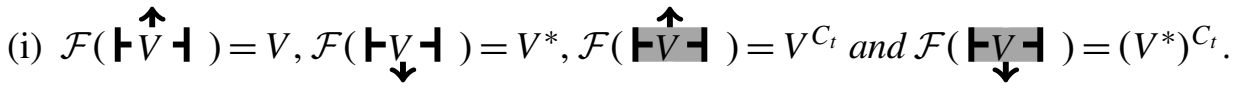

(ii) On unshaded caps and cups $\mathcal{F}$ agrees with $\mathcal{F}^{\prime}$.

(iii) The topological half-twist on $n$ ribbons is sent to rev $\circ \Delta^{n}(t)$ acting on $\mathcal{F}$ of the bottom object, regardless of shading and orientation.

Furthermore, the restriction of $\mathcal{F}$ to $\mathcal{R} \mathcal{I B B O} \mathcal{N}\left(\mathcal{H}\right.$-rep) agrees with $\mathcal{F}^{\prime}$. 
The proof of Theorem 4.11 requires several lemmas, and will occupy the rest of this section.

Comment 4.12. Like $\mathcal{F}^{\prime}, \mathcal{F}$ is not a strict rigid functor. In particular, we have

$$
\mathcal{F}\left(\mathfrak{F}_{\Downarrow} \mathfrak{-}\right)=\left(V^{*}\right)^{C_{t}},
$$

which is not equal to $\left(V^{C_{t}}\right)^{*}$ (although these two representations are isomorphic). In fact, $\left(V^{*}\right)^{C_{t}}$ is equal to the right dual ${ }^{*}\left(V^{C_{t}}\right)$. To see why, notice that, as vector spaces, both $\left(V^{*}\right)^{C_{t}}$ and $*\left(V^{C_{t}}\right)$ are equal to $\operatorname{Hom}_{F}(V, F)$. Each comes with a chosen action of $\mathcal{H}$, and, using the fact that $C_{t}$ is a coalgebra antiautomorphism, one can show that these two actions are identical.

Comment 4.13. A good mnemonic for remembering what $\mathcal{F}$ does to objects is to think of the ribbon as always being labeled with a representation on its light side, so that when you look at the dark side you see that label rotated 180 degrees around the $y$ axis. For example, instead of $V_{i}^{C_{t}}$ you would see ${ }_{i} V$. This works for remembering how duals interact with half twists since when you rotate $V_{i}^{*}$ by 180 degrees about the $y$ axis you see ${ }_{i}^{*} V$.

Comment 4.14. There is more than one extension of $\mathcal{F}^{\prime}$ from $\mathcal{R} \mathcal{I B B O} \mathcal{N}(\mathcal{H}$-rep) to the larger category $\mathcal{H} \mathcal{R} \mathcal{I B}(\mathcal{H}$-rep), but only one satisfies Theorem 4.11(iii).

We first prove the following different characterization of $\mathcal{F}$. This is simpler to prove, and is also more general than Theorem 4.11. However, this result is less satisfying in other ways. For instance, Proposition 4.15 does not imply that all half twists are sent to $t$ acting on $\mathcal{F}$ of the bottom object.

Proposition 4.15. Let $(\mathcal{H}, R, v)$ be a ribbon Hopf algebra, and let $t \in \mathcal{H}$ be an invertible element. There is a unique monoidal functor $\mathcal{F}$ from $\mathcal{H} \mathcal{R} \mathcal{I}(\mathcal{H}$-rep) to $\mathcal{H}$-rep such that:

(i) $\mathcal{F}$ agrees with $\mathcal{F}^{\prime}$ on the full subcategory $\mathcal{R} \mathcal{I B B O} \mathcal{N}(\mathcal{H}$-rep).

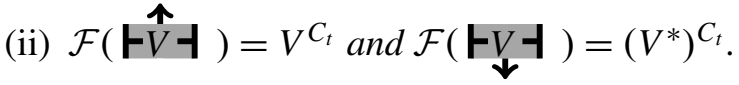

(iii) $\mathcal{F}(Y)=t$ as a morphism from $V$ to $V^{C_{t}}$ or from $V^{*}$ to $\left(V^{*}\right)^{C_{t}}$ depending on orientation.

$\mathcal{F}\left(\nearrow^{\prime}\right)=t^{-1}$ as a morphism from $V^{C_{t}}$ to $V$ or from $\left(V^{*}\right)^{C_{t}}$ to $V^{*}$ depending on orientation (notice that this is a negative half-twist, hence the use of $t^{-1}$ ).

Proof. This is the simplest case of Reshetikhin and Turaev's [1990] more general extension of $\mathcal{F}^{\prime}$ to ribbon graphs with coupons, where we think of the half-twists defined above as coupons on a single ribbon. So we just sketch the proof. Every morphism in $\mathcal{H} \mathcal{R} \mathcal{I}(\mathcal{H}$-rep) is isotopic to ribbon in the following standard form: every shaded object at the bottom has a positive half-twist next to it, then in the 
middle of the diagram there's a ribbon tangle, then at the top of the diagram every shaded object has a negative half-twist next to it. Furthermore two such diagrams are isotopic if and only if the ribbon tangles in the middle of their standard diagrams are isotopic. Hence there is at most one possible functor $\mathcal{F}$. That $\mathcal{F}$ is in fact a functor follows from the fact that $t$ and $t^{-1}$ are inverses of each other, so that composition of diagrams is just given by composing their middle parts.

The following lemma shows how $\mathcal{F}$ acts on those elementary morphisms not already specified in the statement of Proposition 4.15.

Lemma 4.16. Let $(\mathcal{H}, t)$ be a half-ribbon Hopf algebra, and define $\mathcal{F}$ as in Proposition 4.15.

(i) $\mathcal{F}$ sends any positive half-twist to t acting on $\mathcal{F}$ of the bottom object regardless of orientation or shading. $\mathcal{F}$ sends any negative half-twist to $t^{-1}$ regardless of orientation or shading.

(ii) $\mathcal{F}$ takes any simple crossing to Flip $\circ R$ acting on $\mathcal{F}$ of the bottom object, regardless of orientations and shadings.

(iii) $\mathcal{F}$ sends shaded caps and cups to the following maps:

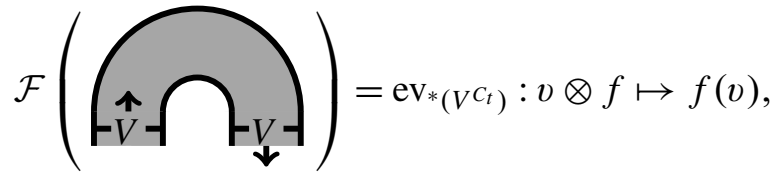

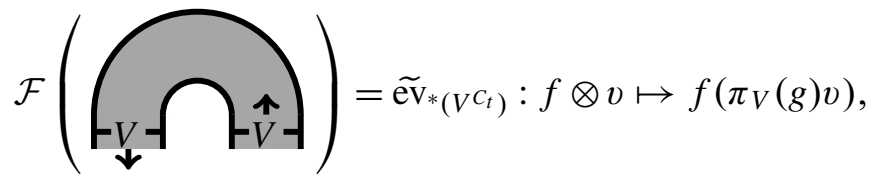

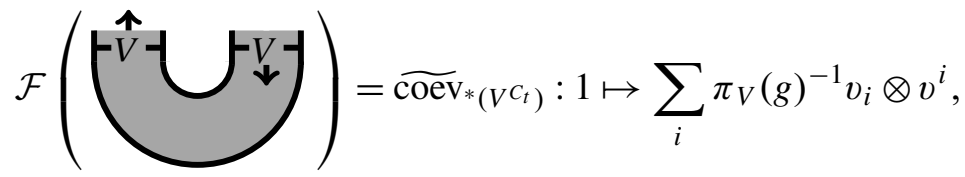

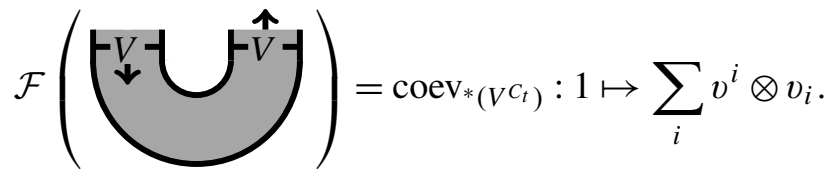

For the explicit formulas, we have used the fact that $V^{C_{t}}$ and $\left(V^{*}\right)^{C_{t}}$ are identical to $V$ and $V^{*}$, respectively, as vector spaces, and chosen dual basis $v_{i}$ and $v^{i}$ for $V$ and $V^{*}$.

Comment 4.17. In order to apply the maps $\operatorname{ev}_{*}\left(V^{C_{t}}\right)$, etc. above, one must use the canonical isomorphism $\left({ }^{*}\left(V^{C_{t}}\right)\right)^{*} \cong V^{C_{t}}$, and the equality $\left(V^{*}\right)^{C_{t}}={ }^{*}\left(V^{C_{t}}\right)$ from Comment 4.12. It is a straightforward exercise to show that these maps are given in coordinates by the above formulas. 
Proof of Lemma 4.16. We prove each of these formulas by a direct calculation.

For the half-twists and ribbon full-twists we only use that $t^{2}=v^{-1}$. For example, the positive half-twist going from unshaded to shaded is a composition of the negative half-twist with the full twist. So this result follows from $t^{-1} v^{-1}=t$.

The formulas for $\mathcal{F}$ applied to a crossing with one or both of the ribbons shaded all follow from the naturality of the braiding. For example, to find $\mathcal{F}$ of a crossing where one strand is shaded, consider the following isotopy:

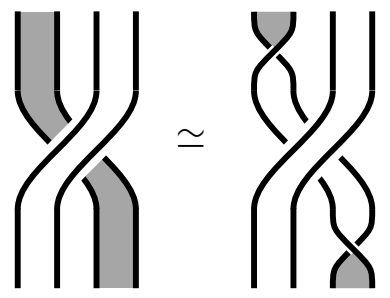

Thus $\mathcal{F}$ of the left side must be equal to $(t \otimes 1) \circ$ Flip $\circ R \circ\left(1 \otimes t^{-1}\right)$. By the naturality of the braiding,

$(t \otimes 1) \circ$ Flip $\circ R \circ\left(1 \otimes t^{-1}\right)=$ Flip $\circ R \circ(1 \otimes t) \circ\left(1 \otimes t^{-1}\right)=$ Flip $\circ R$.

This holds independently of the orientations of the ribbons. A similar argument gives the same formula for crossings where the other ribbon is shaded, or where both are shaded.

It remains to compute the formula for shaded cups and caps. These equations use $g=S(t) t^{-1}=S\left(t^{-1}\right) t$. We explicitly show two of the four cases. The other two are similar.

By the definition of $\mathcal{F}$, one has

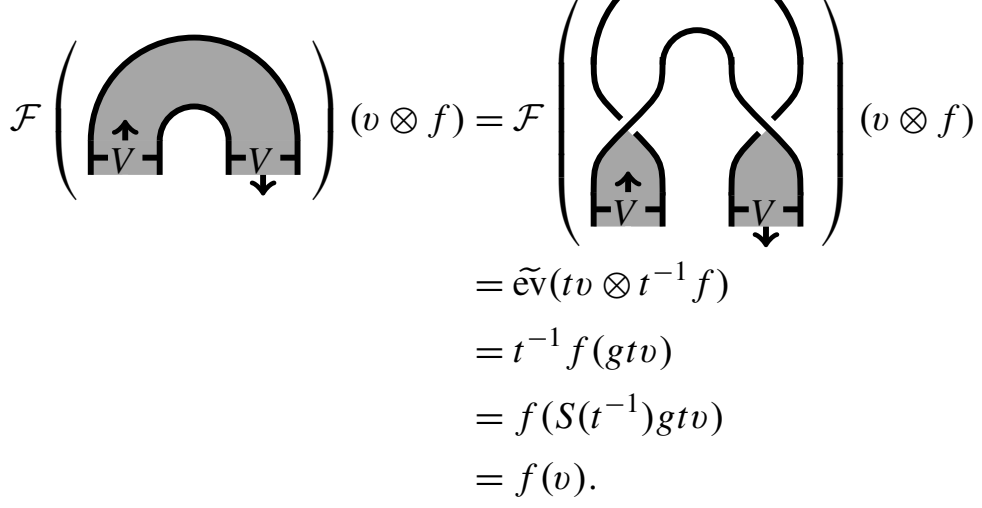

Here (24) is an isotopy, (25) follows from functoriality and our computation of $\mathcal{F}$ on all half-twists, (26) is the definition of $\widetilde{\mathrm{vv}},(27)$ is the definition of the action on the dual space, and (28) uses the formula $g=S(t) t^{-1}$. 
Now for a cup. Notice that, if $e^{i} \in V^{*}$ and $e_{j} \in V$ are dual bases and $x$ is an invertible element in $\mathcal{H}$, then $v^{i}=x e^{i}$ and $v_{i}=S(x)^{-1} e_{i}$ are also dual bases. By the definition of $\mathcal{F}$, we have:
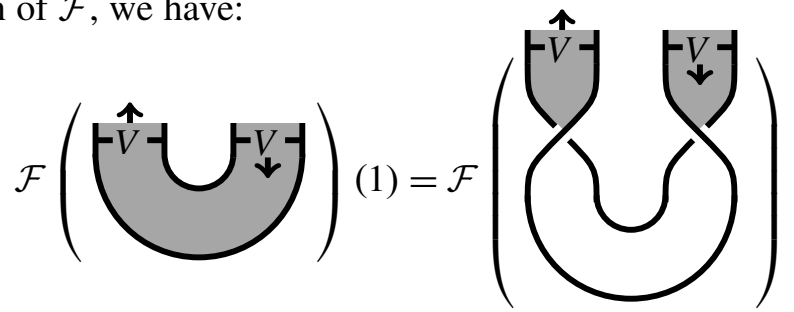

$$
\begin{aligned}
& =\sum t e_{i} \otimes t^{-1} e^{i} \\
& =\sum t S\left(t^{-1}\right) v_{i} \otimes v^{i} \\
& =\sum g^{-1} v_{i} \otimes v^{i} .
\end{aligned}
$$

Here (29) is an isotopy, (30) follows from functoriality and our computation of $\mathcal{F}$ on all half-twists, (31) is the change of dual bases described above with $x=t$, and (31) uses the formula $g=S(t) t^{-1}$.

Note that above we used the formulas relating $t$ to $g$ (which was derived using the formula relating $R$ and $t$ ), but we did not use the formula for $R$ directly. That formula is used in the following proposition which computes how $\mathcal{F}$ acts on the half-twist applied to many strands.

Proposition 4.18. Suppose that $(\mathcal{H}, t)$ is a half-ribbon Hopf algebra, let $\mathcal{F}$ be the unique functor guaranteed by Proposition 4.15. Then, $\mathcal{F}\left(I_{n}\right)=\operatorname{rev} \circ \Delta^{n}(t)$.

Proof. We proceed by induction on $n$, the case $n=1$ being trivial. Consider the isotopy
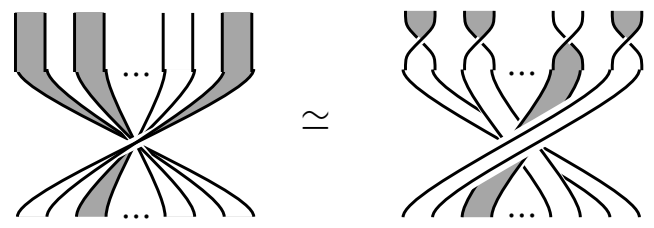

Thus, by the definition of $\mathcal{F}$,

$$
\mathcal{F}\left(I_{n}\right)=(t \otimes t \otimes \cdots \otimes t) \mathcal{F}\left(T_{w_{0}}^{(n)}\right),
$$

where $T_{w_{0}}^{(n)}$ is the braid group element corresponding to the longest element of $S_{n}$. Let $\sigma^{(n)}$ be the braid group element

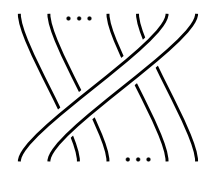


Let $s^{(n)}$ be the image of $\sigma^{(n)}$ in $S_{n}$, and let rev ${ }^{(n)}$ denote the longest element of $S_{n}$. Clearly $T_{w_{0}}^{(n)}=\left(T_{w_{0}}^{(n-1)} \otimes 1\right) \circ \sigma^{(n)}$. Hence

$$
\begin{aligned}
\mathcal{F}\left(I_{n}\right) & =(t \otimes t \otimes \cdots \otimes t) \circ \mathcal{F}\left(T_{w_{0}}^{(n)}\right) \\
& =(t \otimes t \otimes \cdots \otimes t) \circ\left(\mathcal{F}\left(T_{w_{0}}^{(n-1)}\right) \otimes 1\right) \circ \mathcal{F}\left(\sigma^{(n)}\right) \\
& =\left(\mathcal{F}\left(I_{n-1}\right) \otimes t\right) \circ \mathcal{F}\left(\sigma^{(n)}\right) \\
& =\left(\operatorname{rev}^{(n-1)} \Delta^{n-1}(t) \otimes t\right) \circ s^{(n)} \circ\left(1 \otimes \Delta^{n-1}\right)(R) \\
& =\left(\operatorname{rev}^{(n-1)} \Delta^{n-1}(t) \otimes t\right) \circ s^{(n)} \circ\left(1 \otimes \Delta^{n-1}\right)\left(\left(t^{-1} \otimes t^{-1}\right) \Delta(t)\right) \\
& =\operatorname{rev}^{(n)} \Delta^{n}(t) .
\end{aligned}
$$

Here (36) follows from (33) in the case $n-1$, (37) follows from the inductive assumption and the quasitriangularity of $R$, (38) holds because $t$ is a half-ribbon element, and (39) is a straightforward calculation.

This proof works regardless of the orientations and shadings of the ribbons.

Proof of Theorem 4.11. Using the isotopy from (1), the crossing is a composition of the two strand topological half-twist and two copies of the inverse of the one strand topological half-twist. Thus the conditions in the statement uniquely determine $\mathcal{F}$ on all the elementary morphisms listed in the condition of Proposition 4.15. So Proposition 4.15 shows that there is a unique candidate for $\mathcal{F}$. Proposition 4.18 shows that this $\mathcal{F}$ satisfies the remaining condition of the theorem.

\section{Ribbon and half-ribbon elements for $U_{q}(\mathfrak{g})$}

We show that $U_{q}(\mathfrak{g})$ is always a topological half-ribbon Hopf algebra. That is, there exists an element $X$ in a completion of $U_{q}(\mathfrak{g})$ such that $\left(X^{-1} \otimes X^{-1}\right) \Delta(X)$ is the standard $R$-matrix, and $X^{-2}$ is a ribbon element. Interestingly, $X^{-2}$ is not the standard ribbon element $C$. We also classify the different ribbon elements for $U_{q}(\mathfrak{g})$, and discuss how one might decide which of these arise from half-ribbon elements. In particular, we show that the standard ribbon element for $U_{q}\left(\mathfrak{s l}_{2}\right)$ does not arise from a half-ribbon element. We then explain some consequences of varying the ribbon element. It turns out the ribbon element $X^{-2}$ is in some ways particularly nice (see Lemma 5.7 and Section 5E).

5A. A half-ribbon Hopf algebra structure on $U_{q}(\mathfrak{g})$. We first need the following result relating different ribbon elements for the same quasitriangular Hopf algebra.

Lemma 5.1. Let $(\mathcal{H}, R, v)$ be a ribbon Hopf algebra, and $s \in \mathcal{H}$ be a central grouplike element that squares to 1 . Then $(\mathcal{H}, R, v s)$ is also a ribbon Hopf algebra. Furthermore all ribbon elements for $(\mathcal{H}, R)$ are of the form vs for some such $s$.

Proof. This is a straightforward application of the definition of a ribbon element [Barrett and Westbury 1999, Remark 3.4]. 
Theorem 5.2. Let $X$ be the element from Theorem 3.11. Then $\left(U_{q}(\mathfrak{g}), X\right)$ is a topological half-ribbon Hopf algebra. Furthermore $\left(X^{-1} \otimes X^{-1}\right) \Delta(X)$ is the standard $R$-matrix.

Proof. By Theorem 3.11, $\left(U_{q}(\mathfrak{g}), R\right)$ is a quasitriangular Hopf algebra, where $R=\left(X^{-1} \otimes X^{-1}\right) \Delta(X)$ is the standard $R$ matrix. Thus it suffices to show that $X^{-2}$ is a ribbon element for this quasitriangular Hopf algebra. We already know that $\left(U_{q}(\mathfrak{g}), R, C\right)$ is a ribbon Hopf algebra. Thus by Lemma 5.1 it suffices to show that (i) $X^{2} C$ is central; (ii) $X^{2} C$ is grouplike; and (iii) $\left(X^{2} C\right)^{2}=1$.

(i) By Lemma 3.10(iii) and Definition 3.15, $X^{2} C$ acts on $V_{\lambda}$ as multiplication by the scalar $(-1)^{\left\langle 2 \lambda, \rho^{\vee}\right\rangle}$. Thus it is clearly central.

(ii) Any highest weight $v$ for $V_{\lambda} \otimes V_{\mu}$ has weight $\lambda+\mu-\gamma$ for some $\gamma$ in the root lattice. Since $\left\langle 2 \gamma, \rho^{\vee}\right\rangle$ is an even integer for any $\gamma$ in the root lattice, it follows that $X^{2} C$ is grouplike.

(iii) $\left(X^{2} C\right)^{2}$ acts on $V_{\lambda}$ by $(-1)^{2 \cdot\left\langle 2 \lambda, \rho^{\vee}\right\rangle}=1$.

5B. The Frobenius-Schur indicator. We must now discuss a tool for comparing ribbon elements. The following definition of the Frobenius-Schur indicator for a pivotal category was given by [Ng and Schauenburg 2007] (see also [Linchenko and Montgomery 2000] for the case of Hopf algebras and [Fuchs et al. 1999] for $C^{*}$ sovereign categories).

Recall that a pivotal structure is a natural collection of maps $p_{V}: V \rightarrow V^{* *}$ which defines a natural isomorphism Id $\rightarrow * *$ of monoidal functors [Barrett and Westbury 1999]. The category of representations of a ribbon Hopf algebra is always pivotal with the pivotal structure being given by the grouplike element $g=v^{-1} u$ as follows: fix $v \in V$. Then $p_{V}(v)$ is the element of $V^{* *}$ defined by, for all $f \in V^{*}$, $p_{V}(v)(f)=f(g v)$ (see for example [Chari and Pressley 1994]).

Definition 5.3. Given an $F$-linear category with a chosen pivotal structure $p$, define the Frobenius-Schur indicator of an absolutely simple object $V$ as follows: if $V \nsubseteq V^{*}$, then $F S_{p}(V)=0$. Otherwise, choose an isomorphism $f: V \rightarrow V^{*}$. By Schur's lemma there exists some constant, which we define to be $F S_{p}(V)$, such that $f=F S_{p}(V) f^{*} \circ p$.

Comment 5.4. As shown in [Ng and Schauenburg 2007], $F S_{p}(V)$ does not depend on the choice of $f$, and we have $F S_{p}(V)= \pm 1$ (or 0 ).

Comment 5.5. Notice that the Frobenius-Schur indicator depends on the pivotal structure, which in turn depends on the choice of ribbon element. As described in [Ng and Schauenburg 2007], we use $F S_{v}(V)$ to denote the Frobenius-Schur indicator for $V$, calculated with the ribbon element $v$. 
Comment 5.6. The reason for the term Frobenius-Schur indicator is that in the case of groups (and indeed involutory Hopf algebras [Linchenko and Montgomery 2000]) with the trivial pivotal structure, it agrees with the usual Frobenius-Schur indicator, which is 1 when there is an invariant orthogonal form on $V \otimes V,-1$ when there is an invariant symplectic form on $V \otimes V$, and 0 otherwise.

Lemma 5.7. Let $V_{\lambda}$ be an irreducible representation of $U_{q}(\mathfrak{g})$ and assume $V_{\lambda}$ is self-dual. Then $F S_{X^{-2}}\left(V_{\lambda}\right)=1$.

Proof. Let $f: V_{\lambda} \rightarrow V_{\lambda}^{*}$ be an isomorphism. It suffices to show that $f=f^{*} \circ p_{V_{\lambda}}$. Since both $f$ and $f^{*} \circ p_{V_{\lambda}}$ are isomorphisms, it suffices to show that their actions on $v_{\lambda}^{\text {low }}$ agree. But $f\left(v_{\lambda}^{\text {low }}\right)$ is in the lowest weight space of $V_{\lambda}^{*}$, which is onedimensional and pairs nondegenerately with $v_{\lambda}$. Thus, it in fact suffices to show that

$$
f\left(v_{\lambda}^{\text {low }}\right)\left(v_{\lambda}\right)=f^{*} \circ p_{V_{\lambda}}\left(v_{\lambda}^{\text {low }}\right)\left(v_{\lambda}\right) .
$$

Using the formula for $g$ given in Proposition 4.3,

$$
S(X)=X g^{-1}=g X .
$$

Let $k=(-1)^{\left\langle 2 \lambda, \rho^{\vee}\right\rangle} q^{(\lambda, \lambda) / 2+(\lambda, \rho)}$. By Lemma 3.10(ii), $X\left(v_{\lambda}\right)=k\left(v_{\lambda}^{\text {low }}\right)$. Thus the left side of (40) can be simplified as follows

$$
\begin{aligned}
f\left(v_{\lambda}^{\text {low }}\right)\left(v_{\lambda}\right) & =f\left(k^{-1} X\left(v_{\lambda}\right)\right)\left(v_{\lambda}\right) \\
& =k^{-1}\left(X f\left(v_{\lambda}\right)\right)\left(v_{\lambda}\right) \\
& =k^{-1} f\left(v_{\lambda}\right)\left(S(X) v_{\lambda}\right) \\
& =k^{-1} f\left(v_{\lambda}\right)\left(X g^{-1} v_{\lambda}\right) \\
& =k^{-1} f\left(v_{\lambda}\right)\left(g X v_{\lambda}\right) \\
& =k^{-1} f\left(v_{\lambda}\right)\left(k g v_{\lambda}^{\text {low }}\right) \\
& =f\left(v_{\lambda}\right)\left(g v_{\lambda}^{\text {low }}\right) .
\end{aligned}
$$

Here (43) follows because $f$ is an isomorphism, (45) and (46) follow by (41) and (47) by the above formula for the action of $X$ on $v_{\lambda}$.

Now consider the right side of (40). By definition, $p_{V_{\lambda}}\left(v_{\lambda}^{\text {low }}\right)$ is the element of $V_{\lambda}^{* *}$ which takes $\phi \in V_{\lambda}^{*}$ to $\phi\left(g v_{\lambda}^{\text {low }}\right)$. Thus

$$
f^{*} \circ p_{V_{\lambda}}\left(v_{\lambda}^{\text {low }}\right)\left(v_{\lambda}\right)=f\left(v_{\lambda}\right)\left(g v_{\lambda}^{\text {low }}\right) .
$$

We have shown that the two sides of (40) agree, so the result follows.

Turaev [1994] calls the property that Lemma 5.7 holds unimodality and requires it when defining geometric $3 \mathrm{j}$ and $6 \mathrm{j}$ symbols. 
Lemma 5.8. $F S_{C}(V)$ is the usual Frobenius-Schur indicator of the corresponding classical representation.

Proof. Since the Frobenius-Schur indicator is a discrete invariant, it doesn't change under continuous deformation.

5C. Which ribbon elements for $U_{q}(\mathfrak{g})$ arise from half-ribbon elements? Here we classify ribbon elements for $U_{q}(\mathfrak{g})$. We then prove that the standard ribbon element for $U_{q}\left(\mathfrak{s l}_{2}\right)$ is not equal to $t^{-2}$ for any half-ribbon element $t$.

Definition 5.9. Suppose that $\phi$ is a character of $P / Q$, where $P$ is the weight lattice of $\mathfrak{g}$, and $Q$ is the root lattice. Define $s(\phi) \in \widetilde{U_{q}(\mathfrak{g})}$ to act on $V_{\lambda}$ as multiplication by the scalar $\phi(\lambda)$.

Theorem 5.10. The central grouplike elements of $\widetilde{U_{q}(\mathfrak{g})}$ are precisely the $s(\phi)$.

Proof. Suppose that $s$ is a central grouplike element of $\widetilde{U_{q}(\mathfrak{g})}$. Since it is central, $s$ acts by a scalar on any irreducible representation. Furthermore, since it is grouplike, $s$ acts by the same scalar on any two irreducible representations that appear in the same arbitrary tensor product of irreducible representations. Thus such an $s$ gives a function of $P / \sim$, where $\sim$ is the equivalence relation on weights generated by $\lambda \sim \mu$ if $V_{\lambda}$ and $V_{\mu}$ appear in the same tensor product of irreducible representations. It suffices to show that $\lambda \sim \mu$ if and only if $\lambda+Q=\mu+Q \in P / Q$. One can easily see that $\sim$ respects the additive structure of the weight lattice, so it is enough to prove that $V_{\lambda}$ appears in some tensor product also containing the trivial representation $V_{0}$ precisely when $\lambda$ is in the root lattice.

Since the Littlewood-Richardson coefficients are the same in the quantum and classical cases, this reduces to the same question in the classical case. So let $G$ be the simply connected Lie group attached to $U(\mathfrak{g})$, and let $Z(G)$ be the center of $G$. The central character map gives a pairing between $Z(G)$ and the weight lattice mod the root lattice. Since any tensor product of irreducible representations has a well-defined central character, we see that $V_{\lambda}$ is equivalent to $V_{0}$ only if $\lambda$ is in the root lattice.

To see the other direction, let $V$ be a faithful representation of the compact adjoint form $K$ of $G$.

Claim: For any $\lambda$ in the root lattice, there exists $N$ so that, for all $n>N, V_{\lambda}$ occurs as a subrepresentation of $V^{\otimes n}$.

Proof of claim: since $\lambda$ is in the root lattice, $V_{\lambda}$ descends to a representation of $K$. On this compact form we can use character theory. Because $K$ has no center, $\left|\chi_{V}(g)\right|<\operatorname{dim} V$ for any $g \neq 1$. Let

$$
k_{n}=\left|\int_{K} \chi_{V}^{n} d \mu\right|
$$


where $d \mu$ is the normalized Haar measure. Since $K$ is compact and $\left|\chi_{V}(g)\right|<$ $\chi_{V}(1)$ for all $g \neq 1$, we see that

$$
\lim _{n \rightarrow \infty} \frac{\left\langle\chi_{V^{\otimes n}}, \chi_{V_{\lambda}}\right\rangle}{k_{n}}=\lim _{n \rightarrow \infty} \frac{1}{k_{n}} \int_{K} \chi_{V}(g)^{n} \chi_{V_{\lambda}}\left(g^{-1}\right) d \mu=\chi_{V_{\lambda}}(1)=\operatorname{dim} V_{\lambda} .
$$

In particular, for $n \geq N$ we see that $\left\langle\chi_{V^{\otimes n}}, \chi_{V_{\lambda}}\right\rangle$ is nonzero, so $V_{\lambda}$ occurs in $V^{\otimes n}$.

Applying the above fact to 0 and an arbitrary root vector $\lambda$, we see that for a sufficiently high power $N^{\prime}=\max \left(N_{0}, N_{\lambda}\right)$ the tensor power $V^{\otimes N}$ contains both $V_{0}$ and $V_{\lambda}$, and hence $0 \sim \lambda$.

Theorem 5.11. Ribbon elements of $\widetilde{U_{q}(\mathfrak{g})}$ are exactly elements of the form

$$
s(\phi) X^{-2},
$$

where $\phi$ is a character of the weight lattice mod the root lattice of order $\leq 2$.

Proof. This follows from Lemma 5.1 and Theorem 5.10.

We have the following relationship between ribbon elements and the FrobeniusSchur indicator.

Proposition 5.12. If $V_{\lambda}$ is self dual, then $F S_{s(\phi) X^{-2}}\left(V_{\lambda}\right)=\phi(\lambda)$.

Proof. The only part of the definition of the Frobenious-Schur indicator that changes when you change the ribbon element from $X^{-2}$ to $s(\phi) X^{-2}$ is $p_{V_{\lambda}}$, and this is multiplied by $\phi(\lambda)$. Hence the proposition follows from Lemma 5.7.

We wish to understand which of these ribbon element extend to a half-ribbon elements on $\widetilde{U_{q}(\mathfrak{g})}$. As we shall see, the ratio of two half-ribbon elements is grouplike, so to classify all of them we need to understand the grouplike elements in $\widetilde{U_{q}(\mathfrak{g})}$. In general this seems to pose some technical challenges, but we can do the case of $U_{q}\left(\mathfrak{s l}_{2}\right)$.

Proposition 5.13. Let $(\mathcal{H}, R)$ be a quasitriangular Hopf algebra, and assume that $t_{1}$ and $t_{2}$ are two half-ribbon elements. Then $t_{1} t_{2}^{-1}$ is grouplike. (Note that we do not assume here that $t_{1}^{-2}=t_{2}^{-2}$.)

Proof. This follows from the equality $\left(t_{1}^{-1} \otimes t_{1}^{-1}\right) \Delta\left(t_{1}\right)=\left(t_{2}^{-1} \otimes t_{2}^{-1}\right) \Delta\left(t_{2}\right)$.

Lemma 5.14. Let $V$ be the standard representation of $U_{q}\left(\mathfrak{s l}_{2}\right)$, with basis $\left\{v_{+}, v_{-}\right\}$ such that $E\left(v_{-}\right)=v_{+}, K v_{+}=q v_{+}$, and $K v_{-}=q^{-1} v_{-}$. Then $V \otimes V$ contains a copy of the trivial representation $V_{0}$. Furthermore, this is spanned by the element

$$
v_{+} \otimes v_{-}-q^{-1} v_{-} \otimes v_{+} .
$$

Proof. It suffices to check that $\Delta(E)\left(v_{+} \otimes v_{-}-q^{-1} v_{-} \otimes v_{+}\right)=0$. This follows from the definition of $\Delta$. 
Definition 5.15. Let $a \in \mathbb{C}(q)$. Define $K_{a}$ to be the element of $\widetilde{U_{q}\left(\mathfrak{s l}_{2}\right)}$ that acts on any weight vector $v \in V_{\lambda}$ by $K_{a}(v)=a^{\mathrm{wt}(v)}$. Note that $K_{a}$ is grouplike.

Lemma 5.16. All grouplike elements in $\widetilde{U_{q}\left(\mathfrak{s L}_{2}\right)}$ are of the form $K_{a}$, for $a \in \mathbb{C}(q)$.

Proof. A grouplike element is determined by its action on the fundamental representation $V$. Suppose that $\sigma$ is grouplike. Define constants $a, b, c$ and $d$ by $\sigma v_{+}=a v_{+}+b v_{-}$and $\sigma v_{-}=c v_{+}+d v_{-}$. Since $\sigma$ is grouplike it must act trivially on the trivial subrepresentation of $V \otimes V$. Thus by Lemma 5.14,

$$
\begin{aligned}
v_{+} \otimes v_{-}-q^{-1} v_{-} \otimes v_{+} & =\sigma \otimes \sigma\left(v_{+} \otimes v_{-}-q^{-1} v_{-} \otimes v_{+}\right) \\
& =a c\left(1-q^{-1}\right) v_{+} \otimes v_{+}+\left(a d-q^{-1} b c\right) v_{+} \otimes v_{-} \\
& -q^{-1}(a d-q b c) v_{-} \otimes v_{+}+\left(1-q^{-1}\right) b d v_{-} \otimes v_{-} .
\end{aligned}
$$

Comparing coefficients we see that $a c=b d=b c=0$ and $a d=1$. Hence $\sigma$ acts on $V$ in exactly the same way that $K_{a}$ does. Since they are both grouplike and $V$ is a tensor generator for the category of representations of $U_{q}\left(\mathfrak{s l}_{2}\right)$, we see that $\sigma=K_{a}$.

Theorem 5.17. There is no topological half-ribbon element $t$ for $U_{q}\left(\mathfrak{s l}_{2}\right)$ such that $t^{-2}$ is the usual ribbon element $C$.

Proof. By Theorem 3.16, $X$ is a half-ribbon element for $\left(U_{q}\left(\mathfrak{s l}_{2}\right), R\right)$. By Proposition 5.13, any other half-ribbon element is of the form $X \alpha$ for some grouplike element $\alpha$. By Lemma 5.16, $\alpha=K_{a}$ for some $a \in \mathbb{C}(q)$. Since $X$ sends the $\lambda$ weight space to the $-\lambda$ weight, it follows that $K_{a} X K_{a} v=X v$ for any weight space $v$. Therefore, we have that $\left(X K_{a}\right)^{-2}=X^{-2}$. Hence every half-ribbon element $t$ for $U_{q}\left(\mathfrak{s l}_{2}\right)$ has $t^{-2}=X^{-2}$. But $X^{-2}$ acts as $-C$ on the standard representation, so is not equal to $C$.

5D. A summary of ribbon and half-ribbon elements for $U_{q}\left(\mathfrak{s l}_{2}\right), U_{q}\left(\mathfrak{s l}_{3}\right)$ and $\boldsymbol{U}_{\boldsymbol{q}}\left(\mathfrak{s l}_{4}\right)$. We now describe the possible ribbon elements of these three quantum groups, and describe which are the squares of half-ribbon elements. Notice that the three cases all behave differently.

- $U_{q}\left(\mathfrak{s l}_{2}\right)$. There are two ribbon elements $C$, and $X^{-2}$. Only $X^{-2}$ can be realized using a half-ribbon element (see Theorem 5.17).

- $U_{q}\left(\mathfrak{s l}_{3}\right)$. In this case $P / Q \cong \mathbb{Z} / 3$. Thus, there are no central grouplikes of order two, so there can be only one ribbon element. This unique ribbon element can be realized using the half-ribbon element $X$.

- $U_{q}\left(\mathfrak{s l}_{4}\right)$. In this case $P / Q \cong \mathbb{Z} / 4$. This has two characters of order $\leq 2$, so there are two ribbon elements. In this case both come from half-ribbon elements. A straightforward calculation on the standard representation of $U_{q}\left(\mathfrak{s l}_{4}\right)$ shows that $X^{-2}$ is not the standard ribbon element. To see the other half-ribbon element, 
consider the central element $s \in \widetilde{U_{q}\left(\mathfrak{s l}_{4}\right)}$ which acts on $V_{\lambda}$ as multiplication by $i^{4(\lambda, \lambda)}$, where $i$ is the complex fourth root of unity. Recall that $(\lambda, \alpha) \in \mathbb{Z}$ for any root $\alpha$, which implies that $s$ is grouplike. Clearly we also have $s^{4}=1$, and from these two facts it follows that $X^{\prime}:=s X$ is a half-ribbon element for $\left(U_{q}\left(\mathfrak{s l}_{4}\right), R\right)$. One can check that $s$ acts as multiplication by $-i$ on the standard representation, so $X^{\prime-2}=s^{2} X^{-2} \neq X^{-2}$. Since $X^{-2}$ is the nonstandard ribbon element, $X^{\prime-2}$ must be the standard ribbon element.

5E. Varying the ribbon element and diagrammatic categories. Often you'll find a result in the literature saying that a certain diagram category "is the same as" as certain category coming from quantum groups (for example, [Ohtsuki 2002, Appendix H] and [Kuperberg 1996, page 11]). However, in the details of this claim there's an annoying sign difference between the diagram category and the quantum group category. The reason for this is that, although the two categories match up as braided tensor categories, they are different as pivotal categories. This can be fixed by changing the ribbon element on the quantum group side, thus changing the pivotal structure.

We illustrate this here by considering the case of $U_{q}\left(\mathfrak{s L}_{2}\right)$-rep and the TemperleyLieb category (see Section 3E). In the correspondence discussed in [Ohtsuki 2002, Appendix $\mathrm{H}]$, the standard representation of $U_{q}\left(\mathfrak{s l}_{2}\right)$ corresponds to the elementary object (i.e. a single $\bullet$ ) in the Temperley-Lieb category. We now use the FrobeniusSchur indicator to show that, for any such statement to hold on the level of pivotal categories, one must use the ribbon element $X^{-2}$.

Proposition 5.18. Let $\bullet$ denote a single point in Temperley-Lieb (with the trivial pivotal structure discussed in Section $3 E)$. Then $F S(\bullet)=1$.

Proof. The single strand is an isomorphism between $\bullet$ and $\bullet *=\bullet$. The dual of the single strand (given by rotating 180 degrees about the $z$ axis) is again the single strand.

Proposition 5.19. Let $V$ be the standard representation of $U_{q}\left(\mathfrak{s l}_{2}\right)$. Then

$$
F S_{C}(V)=-1 \text { and } F S_{X^{-2}}(V)=1 .
$$

Proof. This is a straightforward calculation using the definitions of $C$ (Definition 3.15) and Lemma 3.10(iii). It also follows from the discussion in Section 5C.

If $\bullet$ in Temperley-Lieb is going to correspond to the standard representation, the Frobenius-Schur indicators should agree. Clearly this can only happen if we use the ribbon element $X^{-2}$.

Comment 5.20. A similar difficulty arises in relating the type $C$ quantum group knot invariants with (a specialization of) the Kauffman polynomial. Once again, one can fix the problem by switching to the ribbon element $X^{-2}$. 
Comment 5.21. This difficulty can also be addressed by changing the diagrammatic category. The Temperley-Lieb category has another pivotal structure $p$ such that $p_{\bullet}=-\mathrm{id}_{\bullet}$. With this pivotal structure $F S(\bullet)=-1$. This other pivotal structure is given diagrammatically by the disoriented Temperley-Lieb category of [Clark, Morrison and Walker 2009].

5F. The effect of varying the ribbon element on knot invariants. Suppose that $(\mathcal{H}, R, v)$ is a ribbon Hopf algebra over a base field $F$. Fix a simple $\mathcal{H}$-module $V$. The functor from Theorem 4.7 sends a link with every component labeled with $V$ to an element of $F$, denoted by $\mathcal{F}^{V}(L)$, which is an invariant of framed oriented links. Note that $\mathcal{F}^{V}(L)$ actually depends on the choice of ribbon element $v$, so when we need to be clear about which ribbon element we are using we will denote it by $\mathcal{F}_{v}^{V}(L)$. Since $V$ is simple the ribbon element acts by a scalar $\theta_{v}(V)$ on $V$. It follows as in, for example, [Ohtsuki 2002, Section 3.3] that $\theta_{v}(V)^{w(L)} \mathcal{F}_{v}^{V}(L)$ is an invariant of oriented but unframed links (where $w(L)$ is the writhe of the link diagram, as described in, for example, [Ohtsuki 2002, page 11] and the current Wikipedia entry for "Writhe").

Proposition 5.22. Suppose that $\mathcal{H}$ is a quasitriangular Hopf algebra with

$$
\operatorname{End}(\mathbf{1})=F,
$$

and that $V$ is a simple $\mathcal{H}$-module. Suppose that $v_{1}$ and $v_{2}$ are two different ribbon elements for $\mathcal{H}$. Let $\theta_{v_{1}}(V)$ and $\theta_{v_{2}}(V)$ be the scalars by which $v_{1}$ and $v_{2}$ act on $V$. Let $\mathcal{F}_{v_{1}}^{V}$ and $\mathcal{F}_{v_{2}}^{V}$ be the functors attached to these two ribbon Hopf algebras. Then for any link $L$,

$$
\theta_{v_{1}}^{w(L)} \mathcal{F}_{v_{1}}^{V}(L)=\left(\frac{F S_{v_{1}}(V)}{F S_{v_{2}}(V)}\right)^{\# L} \theta_{v_{2}}^{w(L)} \mathcal{F}_{v_{2}}^{V}(L)
$$

where \# $L$ is the number of components of $L$.

Proof. By Theorem 5.11, $\alpha:=v_{1} / v_{2}$ is central grouplike element of order 2. Let $\alpha_{V}$ be the scalar by which $\alpha$ acts on $V$. By Proposition 5.12,

$$
\alpha_{V}=F S_{v_{1}}(V) / F S_{v_{2}}(V) .
$$

Let $g_{i}=v_{i}^{-1} u$ where $u$ is the Drinfeld element. It follows immediately from definitions that $\widetilde{\mathrm{ev}}_{2}=\alpha_{V}^{-1} \widetilde{\mathrm{ev}}_{1}$ and $\widetilde{\operatorname{coev}_{2}}=\alpha_{V}^{-1} \widetilde{\operatorname{coev}}_{1}$.

The only elementary morphisms for which $\mathcal{F}_{v_{1}}^{V}$ and $\mathcal{F}_{v_{2}}^{V}$ disagree are left-going cups (corresponding to $\widetilde{\mathrm{coev}}$ ), left-going caps (corresponding to $\widetilde{\mathrm{ev}}$ ), and full-twists. Thus,

$$
\theta_{v_{1}}^{w(L)} \mathcal{F}_{v_{1}}^{V}(L)=\left(\alpha_{V}\right)^{-N_{L}} \theta_{v_{2}}^{w(L)} \mathcal{F}_{v_{2}}^{V}(L),
$$

where

$$
N_{L}:=\#\{\text { left going caps and cups }\}+w(L) .
$$


It is easy to see that $N_{L}(\bmod 2)$ is an invariant of oriented links (for example, check all the Turaev moves [Ohtsuki 2002, Section 3.2]). Furthermore, $N_{L}$ (mod 2) doesn't change when you replace a positive crossing by a negative crossing. Since every link can be unknotted by replacing positive crossings with negative crossings, we see that $N_{L}(\bmod 2)$ depends only on the number of components of $L$. By looking at the $k$-component unlink we see that $N_{L} \equiv \# L(\bmod 2)$.

\section{Questions}

Question 1. Which ribbon Hopf algebras can be endowed with a half-ribbon element?

There are several aspects to this question. One could look for examples of halfribbon Hopf algebras other than $U_{q}(\mathfrak{g})$. One could also try to find nonexamples, in the sense of finding ribbon Hopf algebras that do not contain the required element $t$. Such examples exist (at least for topological ribbon Hopf algebras). For instance we showed in Section $5 \mathrm{C}$ that $U_{q}\left(\mathfrak{s l}_{2}\right)$, with the standard ribbon element, cannot be made into a half-ribbon Hopf algebra. However, it can be modified by multiplying the ribbon element by a central grouplike element, and then it does have the required $t$. One could also ask if there are examples of ribbon Hopf algebras that cannot be made into half-ribbon Hopf algebras, even allowing this sort of modification. More ambitiously, one could look for a general method of determining when a ribbon Hopf algebra $\mathcal{H}$ contains an element $t$ such that $(\mathcal{H}, t)$ is a half-twist Hopf algebra.

In the current work we have mainly considered $U_{q}(\mathfrak{g})$, which is infinite-dimensional, and only has a topological half-ribbon element in the sense that $t$ only belongs to a completion of $U_{q}(\mathfrak{g})$. We feel it would be interesting to look at the case of finite-dimensional Hopf algebras as well.

Question 2. Fix a Hopf algebra $\mathcal{H}$. Is there a natural set of conditions one can impose on an element $t \in \mathcal{H}$ which guarantees that $(\mathcal{H}, t)$ is a half-ribbon Hopf algebra?

We would like to be able to start with a Hopf algebra, which is not a priori quasitriangular, and endow it with a ribbon (and half-ribbon) structure by finding a certain $t \in \mathcal{H}$. One can of course write down the conditions $t$ needs to satisfy by insisting that $\left(t^{-1} \otimes t^{-1}\right) \Delta(t)$ is a quasitriangular structure, and $t^{-2}$ is a ribbon element. However, these are very difficult to deal with, so the real question is to find nicer conditions on $t$. This would give a new method of constructing quasitriangular Hopf algebras.

Question 3. What happens if you weaken the conditions on $t$ ? 
Checking that $\left(t^{-1} \otimes t^{-1}\right) \Delta t$ is a quasitriangular structure is difficult, but for some applications one can weaken this condition by insisting only that

(i) for any representation $V$ and $W$ of $\mathcal{H}$, the map

$$
\text { Flip } \circ\left(t^{-1} \otimes t^{-1}\right) \Delta(t): V \otimes W \rightarrow W \otimes V
$$

is an isomorphism; and

(ii) $t^{-2}$ acts as a ribbon element, in the sense that

$$
t^{-2} \circ \text { mult } \circ(S \otimes 1)\left(\left(t^{-1} \otimes t^{-1}\right) \Delta(t)\right)
$$

is grouplike, and so can be used to develop a theory of quantum trace.

These conditions seem easier to check, and a Hopf algebra $\mathcal{H}$ with such a $t$ already has some nice structure. This could be used, for instance, in studying coboundary categories [Drinfel'd 1989].

\section{Acknowledgments}

We thank Joel Kamnitzer, Scott Morrison, and Nicolai Reshetikhin for helpful conversations. We also thank the anonymous referee for very thorough and helpful comments.

\section{References}

[Barrett and Westbury 1999] J. W. Barrett and B. W. Westbury, "Spherical categories", Adv. Math. 143:2 (1999), 357-375. MR 2000c:18007 Zbl 0930.18004

[Chari and Pressley 1994] V. Chari and A. Pressley, A guide to quantum groups, Cambridge University Press, New York, 1994. MR 95j:17010 Zbl 0839.17009

[Clark, Morrison and Walker 2009] D. Clark, S. Morrison, and K. Walker, "Fixing the functoriality of Khovanov homology”, Geom. Topol. 13:3 (2009), 1499-1582. MR 2496052 Zbl 1169.57012

[Drinfel'd 1989] V. G. Drinfel'd, “Quasi-Hopf algebras”, Algebra i Analiz 1:6 (1989), 114-148. In Russian; translated in Leningrad Math. J. 1:6 (1990), 1419-1457. MR 91b:17016

[Fuchs et al. 1999] J. Fuchs, A. C. Ganchev, K. Szlachányi, and P. Vecsernyés, " $S_{4}$ symmetry of $6 j$ symbols and Frobenius-Schur indicators in rigid monoidal $C^{*}$ categories", J. Math. Phys. 40:1 (1999), 408-426. MR 99k:81111 Zbl 0986.81044

[Goodman and Wenzl 2002] F. Goodman and H. Wenzl, "Ideals in the Temperley Lieb category", preprint, 2002. arXiv 0206301

[Henriques and Kamnitzer 2006] A. Henriques and J. Kamnitzer, "Crystals and coboundary categories”, Duke Math. J. 132:2 (2006), 191-216. MR 2007m:17020 Zbl 1123.22007

[Kamnitzer and Tingley 2009] J. Kamnitzer and P. Tingley, "The crystal commutor and Drinfel'd's unitarized R-matrix", J. Algebraic Combin. 29:3 (2009), 315-335. MR 2496310

[Kirillov and Reshetikhin 1990] A. N. Kirillov and N. Reshetikhin, " $q$-Weyl group and a multiplicative formula for universal $R$-matrices”, Comm. Math. Phys. 134:2 (1990), 421-431. MR 92c:17023 Zbl 0723.17014 
[Kuperberg 1996] G. Kuperberg, "Spiders for rank 2 Lie algebras", Comm. Math. Phys. 180:1 (1996), 109-151. MR 97f:17005 Zbl 0870.17005

[Levendorskiǔ and Soĭbel'man 1991] S. Z. Levendorskiǔ and Y. S. Sořbel'man, "The quantum Weyl group and a multiplicative formula for the $R$-matrix of a simple Lie algebra", Funktsional. Anal. $i$ Prilozhen. 25:2 (1991), 73-76. In Russian; translated in Funct. Anal. Appl. 25:2 (1991), 143-145. MR 93a:17017

[Linchenko and Montgomery 2000] V. Linchenko and S. Montgomery, "A Frobenius-Schur theorem for Hopf algebras", Algebr. Represent. Theory 3:4 (2000), 347-355. MR 2001k:16073 Zbl 0971. 16018

[Lusztig 1993] G. Lusztig, Introduction to quantum groups, Progress in Math. 110, Birkhäuser, Boston, MA, 1993. MR 94m:17016 Zbl 0788.17010

[Ng and Schauenburg 2007] S.-H. Ng and P. Schauenburg, "Higher Frobenius-Schur indicators for pivotal categories", pp. 63-90 in Hopf algebras and generalizations, edited by L. H. Kauffman et al., Contemp. Math. 441, Amer. Math. Soc., Providence, RI, 2007. MR 2008m:18015 Zbl 1153.18008

[Ohtsuki 2002] T. Ohtsuki, Quantum invariants: a study of knots, 3-manifolds, and their sets, Series on Knots and Everything 29, World Scientific, River Edge, NJ, 2002. MR 2003f:57027 Zbl 0991.57001

[Reshetikhin 1989] N. Y. Reshetikhin, "Quasitriangular Hopf algebras and invariants of links", Algebra i Analiz 1:2 (1989), 169-188. In Russian; translated in Leningrad Math. J., 1:2 (1990), 491-513. MR 90k:17033

[Reshetikhin and Turaev 1990] N. Y. Reshetikhin and V. G. Turaev, "Ribbon graphs and their invariants derived from quantum groups", Comm. Math. Phys. 127:1 (1990), 1-26. MR 91c:57016 Zbl 0768.57003

[Reshetikhin and Turaev 1991] N. Reshetikhin and V. G. Turaev, "Invariants of 3-manifolds via link polynomials and quantum groups”, Invent. Math. 103:3 (1991), 547-597. MR 92b:57024 Zbl 0725.57007

[Shum 1994] M. C. Shum, “Tortile tensor categories”, J. Pure Appl. Algebra 93:1 (1994), 57-110. MR 95a:18008 Zbl 0803.18004

[Turaev 1994] V. G. Turaev, Quantum invariants of knots and 3-manifolds, de Gruyter Studies in Math. 18, Walter de Gruyter \& Co., Berlin, 1994. MR 95k:57014 Zbl 0812.57003

Communicated by Susan Montgomery

Received 2008-10-06 Revised 2009-09-11 Accepted 2009-10-17

nsnyder@math.columbia.edu Department of Mathematics, Columbia University, Rm 626, MC 4403, 2990 Broadway, New York, NY 10027, United States http://math.columbia.edu/ nsnyder/

ptingley@math.mit.edu Department of Mathematics, Massachusetts Institute of Technology, 77 Massachusetts Ave, Cambridge, MA 02139, United States http://www-math.mit.edu/ ptingley/ 


\title{
A 2-block splitting in alternating groups
}

\author{
Christine Bessenrodt
}

\begin{abstract}
In 1956, Brauer showed that there is a partitioning of the $p$-regular conjugacy classes of a group according to the $p$-blocks of its irreducible characters with close connections to the block theoretical invariants. In a previous paper, the first explicit block splitting of regular classes for a family of groups was given for the 2-regular classes of the symmetric groups. Based on this work, the corresponding splitting problem is investigated here for the 2-regular classes of the alternating groups. As an application, an easy combinatorial formula for the elementary divisors of the Cartan matrix of the alternating groups at $p=2$ is deduced.
\end{abstract}

\section{Introduction}

Richard Brauer [1956] introduced the idea of not only distributing characters into $p$-blocks but also of associating $p$-regular conjugacy classes to $p$-blocks. He showed that it is possible to distribute the $p$-regular classes into blocks in a way that fits with the blocks of irreducible Brauer characters (and suitable subsets of ordinary irreducible characters in the blocks); this is to say that the determinant of the corresponding block part of the Brauer character table (or a suitable part of the ordinary character table) is not congruent to 0 modulo $\mathfrak{p}$ (a prime ideal over $p$ ). Given such a splitting of $p$-regular classes into blocks, Brauer showed that the elementary divisors of the Cartan matrix of a block are then exactly the $p$-parts in the orders of the centralizers of elements in the classes corresponding to the block. $\mathrm{He}$ also observed that in general there may be several such block splittings, and there did not seem to be any natural choice for a given finite group.

But while it is well known how to determine the $p$-blocks of irreducible characters, for the $p$-regular classes only the existence of such a block splitting is known by Brauer's work - concrete examples for providing such a distribution for families of groups were not known for a long time. Only recently, such an explicit block splitting in the sense of Brauer was exhibited for the conjugacy classes of odd order elements and the 2-blocks of the symmetric groups [Bessenrodt 2007];

MSC2000: primary 20C15; secondary 20C20, $20 \mathrm{C} 30$.

Keywords: alternating groups, $p$-regular conjugacy classes, irreducible characters, Brauer characters, $p$-blocks, Cartan matrix. 
in fact, in this case the 2-block splitting of the 2-regular classes is unique. The proof exploited detailed information on the double covers of the symmetric groups, in particular results on the 2-powers in the spin character values of these groups [Bessenrodt and Olsson 2000] as well as on the 2-block distribution of the spin characters [Bessenrodt and Olsson 1997] turned out to be important ingredients.

Based on these results, the present paper investigates the corresponding problem of constructing a 2-block splitting of the 2-regular classes for the alternating groups. We provide a basic set of characters for the alternating groups, and find a natural choice for a block splitting of the classes. As an application, we deduce an easy combinatorial description of the invariants of the Cartan matrices for the 2-blocks of the alternating groups.

Here is a brief outline of the sections. In Section 2, we recall Brauer's results on block splittings for finite groups which motivated the present work. Then, in Section 3, some combinatorial notations needed in the representation theory of the symmetric groups is introduced, and we state some results from [Bessenrodt 2007] on the block splitting of 2-regular classes for the symmetric groups that are the basis for the new results on alternating groups. In particular, the class labels for the 2-block splitting of $S_{n}$ are recalled. In Section 4, we first collect the necessary information on characters of the alternating groups, and prove some preliminary results towards the construction of a class splitting for the alternating groups. In the main Theorem 4.7 properties of the determinants of the corresponding block character tables are proved which imply that the construction gives indeed a block splitting of the classes. By Brauer's Theorem, our result then implies an easy combinatorial description of the Cartan invariants for the 2-blocks of the alternating groups (Corollary 4.9).

\section{Brauer's block splitting}

Let $G$ be a finite group, $p$ a prime, $(\mathfrak{K}, R, F)$ a $p$-modular splitting system for $G$, and $\mathfrak{p}$ a maximal ideal of $R$ lying over $p$. Let $\ell(G)$ be the cardinality of the set $\mathrm{Cl}_{p^{\prime}}(G)$ of $p$-regular conjugacy classes in $G$. For each $K \in \mathrm{Cl}_{p^{\prime}}(G)$ we let $x_{K}$ denote an element in $K$. A defect group of $K$ is a Sylow $p$-subgroup of $C_{G}(x)$ for some $x \in K$; if this has order $p^{d}$, then $d$ is called the $p$-defect of $K$. We let $\operatorname{IBr}(G)$ denote the set of modular irreducible characters of $G$; then

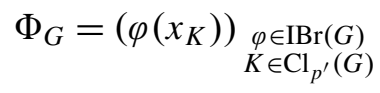

is the Brauer character table of $G$. It is well known by Brauer's work that the Brauer character table is nonsingular modulo $p$, that is,

$$
\operatorname{det} \Phi_{G} \not \equiv 0 \quad(\bmod \mathfrak{p}) .
$$


Furthermore, we let $D=\left(d_{\chi \varphi}\right)_{\chi \in \operatorname{Irr}(G), \varphi \in \operatorname{IBr}(G)}$ denote the $p$-decomposition matrix for $G$, and we let $C=D^{t} D$ denote its Cartan matrix. Let $\mathrm{Bl}_{p}(G)$ be the set of $p$-blocks of $G$. For $B \in \mathrm{Bl}_{p}(G), \operatorname{Irr}(B)$ is the set of ordinary irreducible characters in $B, \operatorname{IBr}(B)$ is the set of modular irreducible characters in $B, \ell(B)=|\operatorname{IBr}(B)|$, $D(B)=\left(d_{\chi \varphi}\right)_{\chi \in \operatorname{Irr}(B), \varphi \in \operatorname{IBr}(B)}$ denotes the $p$-decomposition matrix for $B$ and $C(B)$ is the Cartan matrix for $B$.

Then $C$ resp. $D$ are the block direct sums of the matrices $C(B)$ resp. $D(B)$, $B \in \mathrm{Bl}_{p}(G)$.

The following result was proved by Brauer.

Theorem 2.1. [Brauer 1956, Section 5] There exists a disjoint decomposition of $\mathrm{Cl}_{p^{\prime}}(G)$ into blocks of p-regular conjugacy classes

$$
\mathrm{Cl}_{p^{\prime}}(G)=\bigcup_{B \in \mathrm{Bl}_{p}(G)} \mathrm{Cl}_{p^{\prime}}(B)
$$

and a selection of characters $\operatorname{Irr}^{\prime}(B) \subseteq \operatorname{Irr}(B)$ for each p-block $B$ of $G$ such that the following conditions are fulfilled:

(i) $\left|\mathrm{Cl}_{p^{\prime}}(B)\right|=\left|\operatorname{Irr}^{\prime}(B)\right|=\ell(B)$ for all $B \in \mathrm{Bl}_{p}(G)$;

(ii) For $X_{B}=\left(\chi\left(x_{K}\right)\right){ }_{\chi \in \in \operatorname{Irr}^{\prime}(B)}$, we have $\operatorname{det} X_{B} \not \equiv 0(\bmod \mathfrak{p})$; $K \in \mathrm{Cl}_{p^{\prime}}(B)$

(iii) For $\Phi_{B}=\left(\varphi\left(x_{K}\right)\right)_{\varphi \in \operatorname{IBr}(B)}$, we have $\operatorname{det} \Phi_{B} \not \equiv 0(\bmod \mathfrak{p})$; $K \in \mathrm{Cl}_{p^{\prime}}(B)$

(iv) For $D_{B}=\left(d_{\chi \varphi}\right)_{\substack{\chi \in \operatorname{Irr}^{\prime}(B) \\ \varphi \in \operatorname{IBr}(B)}}$, we have $\operatorname{det} D_{B} \neq \equiv 0(\bmod \mathfrak{p})$.

Furthermore, the elementary divisors of the Cartan matrix $C(B)$ are then exactly the orders of the p-defect groups of the conjugacy classes in $\mathrm{Cl}_{p^{\prime}}(B)$, for all $B$ in $\mathrm{Bl}_{p}(G)$.

The properties in (ii)-(iv) are not independent of each other, as $X_{B}=D_{B} \Phi_{B}$. In particular, if we have a suitable choice $\operatorname{Irr}^{\prime}(B)$ of characters that satisfies (iv), and a suitable choice of classes that satisfies (iii), then these together are a suitable choice for (ii). If we have a basic set of irreducible characters, that is, a subset $\operatorname{Irr}^{\prime}(G) \subseteq \operatorname{Irr}(G)$ giving a $\mathbb{Z}$-basis for the character restrictions to the $p$-regular classes, then the $p$-block decomposition of this set will give a suitable choice of sets $\operatorname{Irr}^{\prime}(B)$ satisfying (iv).

\section{The 2-block splitting for $S_{n}$}

Let $n \in \mathbb{N}$. For the symmetric groups $S_{n}$, the corresponding combinatorial notions and their representation theory, we will follow mostly the usual notation in [James and Kerber 1981]. 
Let $P$ be the set of partitions, $P(n)$ the partitions of $n$. For a partition $\lambda$ of $n$, the number of its (nonzero) parts is called its length, and is denoted by $l(\lambda)$. The complex irreducible character of $S_{n}$ corresponding to $\lambda$ is denoted by [ $\left.\lambda\right]$. For any partition $\mu$ of $n$, we choose an element $\sigma_{\mu}$ in $S_{n}$ of cycle type $\mu$.

Let $\mu=\left(1^{m_{1}(\mu)}, 2^{m_{2}(\mu)}, \ldots\right)$ be written in exponential notation; then we set

$$
a_{\mu}=\prod_{i \geq 1} i^{m_{i}(\mu)}, \quad b_{\mu}=\prod_{i \geq 1} m_{i}(\mu) !
$$

Let $z_{\mu}=\left|C_{S_{n}}\left(\sigma_{\mu}\right)\right|$; then $z_{\mu}=a_{\mu} b_{\mu}$.

Let $p$ be a prime; we will soon fix this to $p=2$. A partition is called $p$-regular if no part is repeated $p$ or more times, and a partition is called $p$-class regular if no part is divisible by $p$.

Let $\mathscr{D}(n)$ be the set of partitions of $n$ into distinct parts; this is thus the set of 2-regular partitions of $n$. Let $O(n)$ be the set of partitions of $n$ into odd parts only; this is the set of 2-class regular partitions of $n$. Let $O=\bigcup_{n \in \mathbb{N}} \mathcal{O}(n)$ and let $\mathscr{D}=\bigcup_{n \in \mathbb{N}} \mathscr{D}(n)$. It is well known that

$$
\operatorname{Irr}^{\prime}\left(S_{n}\right)=\{[\lambda], \lambda \in \mathscr{D}(n)\}
$$

forms a 2-basic set for $S_{n}$.

Then the 2-regular character table of the symmetric group $S_{n}$ is defined to be

$$
X_{n}=\left([\lambda]\left(\sigma_{\alpha}\right)\right)_{\substack{\lambda \in \mathscr{D}(n) \\ \alpha \in \mathcal{O}(n)}}
$$

where the partitions are ordered in a suitable way.

As a special case of a result by Olsson [2003], we know that $\left|\operatorname{det}\left(X_{n}\right)\right|=$ $\prod_{\mu \in \mathscr{O}(n)} a_{\mu}$, and thus in particular,

$$
2 \nmid \operatorname{det}\left(X_{n}\right) .
$$

The main result in [Bessenrodt 2007] provides a block version of this property, by distributing not only the characters but also the 2-regular conjugacy classes into blocks in such a way that the corresponding block parts of the character table have odd determinants. This block distribution of conjugacy classes provided a block splitting in the sense of Brauer as described in the previous section.

We recall this 2-block splitting for the symmetric groups below. The reader is referred to [Bessenrodt 2007] for the full results; these involve more detailed information on spin characters which we omit here since it would require recalling a lot of notation on double cover groups and their characters.

For the combinatorics of the $p$-modular representation theory for $S_{n}$, and in particular the $p$-block distribution of its characters, we refer to [James and Kerber 1981]. 
Let $B$ be a 2-block of $S_{n}$, with associated 2-core $\kappa(B)$; this is then a staircase partition $\rho_{k}=(k, k-1, \ldots, 2,1), k \in \mathbb{N}_{0}$. For any partition $\lambda$, we denote by $\lambda_{(2)}$ the 2-core of $\lambda$. Then we define

$$
\mathscr{D}_{B}=\left\{\lambda \in \mathscr{D}(n) \mid \lambda_{(2)}=\kappa(B)\right\} .
$$

This is the set of labels of irreducible characters in $B$ in the basic set mentioned above, and we set

$$
\operatorname{Irr}^{\prime}(B)=\left\{[\lambda] \mid \lambda \in \mathscr{D}_{B}\right\} .
$$

To define the splitting of the classes we need a few more definitions.

For a partition $\lambda=\left(\lambda_{1}, \ldots, \lambda_{m}\right) \in \mathscr{D}(n)$ we set

$$
\operatorname{dbl}(\lambda)=\left(\left[\frac{\lambda_{1}+1}{2}\right],\left[\frac{\lambda_{1}}{2}\right],\left[\frac{\lambda_{2}+1}{2}\right],\left[\frac{\lambda_{2}}{2}\right], \ldots,\left[\frac{\lambda_{m}+1}{2}\right],\left[\frac{\lambda_{m}}{2}\right]\right),
$$

the doubling of $\lambda$. For example, the staircase $\rho_{k}=(k, k-1, \ldots, 2,1)$ is the doubling of the partition $\tau_{k}=(2 k-1,2 k-5, \ldots)$.

The most natural way of defining the blocks of classes is based on the Glaisher map which we consider next.

J. W. L. Glaisher [1883] defined a bijection between partitions with parts not divisible by a given number $k$ on the one hand and partitions where no part is repeated $k$ times on the other hand; in particular for $k=2$ this gives a bijection between $O(n)$ and $\mathscr{D}(n)$. Here, Glaisher's map $G$ is defined as follows. Let $\alpha=$ $\left(1^{m_{1}}, 3^{m_{3}}, \cdots\right) \in \mathcal{O}(n)$. Write each multiplicity $m_{i}$ as a sum of distinct powers of 2, that is, in its 2-adic decomposition: $m_{i}=\sum_{j} 2^{a_{i j}}$. Then $G(\alpha) \in \mathscr{D}(n)$ consists of the parts $\left(2^{a_{i j}} i\right)_{i, j}$, sorted in order to give a partition.

Let $B$ be a 2-block of $S_{n}$, contained in a 2-block $\widetilde{B}$ of the double cover group $\widetilde{S}_{n}$ (see [Bessenrodt and Olsson 1997] for background and notation). Then we define the set

$$
\mathrm{O}_{B}=\mathscr{O}_{\widetilde{B}}=\left\{\alpha \in \mathcal{O}(n) \mid \mathrm{dbl}(G(\alpha))_{(2)}=\kappa(B)\right\} .
$$

Note that in [Bessenrodt 2007] we have used the language of $\overline{4}$-combinatorics for the description of the 2-block distribution of spin characters, and the set $O_{B}$ is then the set of partitions of type $\mathcal{O}$ such that the Glaisher image has as $\overline{4}$-core the one associated to $\widetilde{B}$.

The sets $O_{B}$ for the 2-blocks $B$ of $S_{n}$ give then a set partition $O(n)=\bigcup_{B} O_{B}$.

The set $O_{B}$ is the set of labels of the 2-regular classes we want to associate to $B$, that is, we set

$$
\mathrm{Cl}^{\prime}(B)=\left\{\sigma_{\alpha}^{S_{n}} \mid \alpha \in O_{B}\right\}
$$

Defining

$$
\mathscr{D}_{\widetilde{B}}=\left\{\lambda \in \mathscr{D}(n) \mid \operatorname{dbl}(\lambda)_{(2)}=\kappa(B)\right\},
$$

the Glaisher map then induces bijections $\mathscr{O}_{B} \rightarrow \mathscr{D}_{\widetilde{B}}$, for all 2-blocks $B$ of $S_{n}$. 
By [Bessenrodt and Olsson 1997], $\left|\mathscr{D}_{B}\right|=\left|\mathscr{D}_{\widetilde{B}}\right|=p(w(B))$. Thus the following block parts of the character table are all square matrices:

$$
X_{B}=\left([\mu]\left(\sigma_{\alpha}\right)\right)_{\substack{\mu \in \mathscr{D}_{B} \\ \alpha \in \mathscr{O}_{B}}} .
$$

Denoting the irreducible Brauer characters of $S_{n}$ by $\varphi^{\mu}, \mu \in \mathscr{D}(n)$, we also consider the corresponding block part of the Brauer character table:

$$
\Phi_{B}=\left(\varphi^{\mu}\left(\sigma_{\alpha}\right)\right)_{\substack{\mu \in \mathscr{D}_{B} \\ \alpha \in \mathbb{O}_{B}}} .
$$

Theorem 3.1. [Bessenrodt 2007] Let $\operatorname{Irr}^{\prime}(B)$ and $\mathrm{Cl}^{\prime}(B)$ for the 2-blocks $B$ of $S_{n}$ be defined as above. Then the determinants

$$
\operatorname{det} \Phi_{B}=\operatorname{det} X_{B}, \quad B \in \operatorname{Bl}\left(S_{n}\right)
$$

of the associated block parts of the character table and the Brauer character table are all odd.

Thus the sets $\mathrm{Cl}^{\prime}(B)$ define a 2-block splitting of the 2-regular classes for $S_{n}$.

Remarks 3.2. (i) More precisely, the determinant $\operatorname{det} X_{B}$ is (up to sign) the odd part of the determinant of the corresponding block part of the reduced spin character table for the 2-block $\tilde{B}$ of the double cover group $\tilde{S}_{n}$ containing $B$, that is,

$$
Z_{S}(\tilde{B})=\left(\langle\lambda\rangle\left(\tilde{\sigma}_{\alpha}\right)\right)_{\substack{\lambda \in \mathscr{D}_{\tilde{B}} \\ \alpha \in \mathscr{O}_{B}}}
$$

See [Bessenrodt 2007] for the notation used here and details on this result.

(ii) By the 2-block splitting for $S_{n}$ given above and Brauer's Theorem, the elementary divisors of the Cartan matrix $C_{B}$ of a 2-block $B$ of $S_{n}$ are exactly the 2-powers

$$
2^{k_{\alpha}}=\left|C_{S_{n}}\left(\sigma_{\alpha}\right)\right|_{2}, \alpha \in \mathcal{O}_{B} .
$$

Here, the 2-defect of the class of type $\alpha$ in $S_{n}$ may easily be computed as follows [Bessenrodt 2007]:

$$
k_{\alpha}=l(\alpha)-l(G(\alpha)) .
$$

This is a restatement of a formula from [Uno and Yamada 2006] which is based on [Bessenrodt and Olsson 1997]; a corrected version of an earlier formula from [Olsson 1986] already appeared in [Bessenrodt and Olsson 1997]. One should note, though, that this formula was used in the confirmation of the block splitting for $S_{n}$ in [Bessenrodt 2007], so this does not give an independent proof for the elementary divisors of the Cartan matrix. 


\section{A 2-block splitting for alternating groups}

We also have to introduce some notation for the alternating group $A_{n}$.

We let $P^{+}(n)=\left\{\lambda \in P(n) \mid(-1)^{n-l(\lambda)}=1\right\}$ denote the set of even partitions in $P(n)$; these are the cycle types of elements in $A_{n}$.

The conjugacy classes in $A_{n}$ are then of two types. The classes labeled by partitions $\mu \in P^{+}(n) \backslash(\mathcal{O} \cap \mathscr{D})(n)$ are the nonsplit classes, that is, those conjugacy classes of $S_{n}$ which are also $A_{n}$-classes; we note that the corresponding $A_{n}$-centralizer is then of order $z_{\mu}^{\prime}=z_{\mu} / 2$. For the partitions $\mu \in(\mathscr{O} \cap \mathscr{D})(n)$, the $S_{n}$-class of $\sigma_{\mu}$ splits into two conjugacy classes in $A_{n}$, for which we denote representatives by $\sigma_{\mu}^{+}$and $\sigma_{\mu}^{-}$; their centralizers are of order $z_{\mu}^{\prime}=z_{\mu}$.

A set of representatives of the 2-regular classes of $A_{n}$ is thus given by:

$$
\mathscr{R}(n)=\left\{\sigma_{\alpha} \mid \alpha \in(\mathscr{O} \backslash \mathfrak{O} \cap \mathscr{D})(n)\right\} \cup\left\{\sigma_{\alpha}^{ \pm} \mid \alpha \in(\mathscr{O} \cap \mathscr{D})(n)\right\} .
$$

Furthermore, we briefly have to recall some information on the irreducible $A_{n}$ characters [James and Kerber 1981, Section 2.5].

For a partition $\lambda$ of $n$, let $\lambda^{\prime}$ denote the conjugate partition. Let

$$
\mathscr{S}(n)=\left\{\lambda \in P(n) \mid \lambda=\lambda^{\prime}\right\}
$$

be the set of symmetric partitions of $n$.

If $\lambda$ is nonsymmetric, then $[\lambda] \downarrow_{A_{n}}=\left[\lambda^{\prime}\right] \downarrow_{A_{n}}$ is irreducible. Let $\{\lambda\}=\left\{\lambda^{\prime}\right\}$ denote this irreducible character of $A_{n}$.

If $\lambda=\lambda^{\prime}$, then $[\lambda] \downarrow_{A_{n}}=\{\lambda\}_{+}+\{\lambda\}_{-}$is a sum of two distinct irreducible $A_{n^{-}}$ characters (which are conjugate in $S_{n}$ ).

This gives all the irreducible complex characters of $A_{n}$, that is,

$$
\operatorname{Irr}\left(A_{n}\right)=\left\{\{\lambda\}_{ \pm} \mid \lambda \in \mathscr{S}(n)\right\} \cup\{\{\lambda\} \mid \lambda \in(P \backslash \mathscr{S})(n)\} .
$$

The characters $\{\lambda\}_{ \pm}$, for $\lambda \in \mathscr{Y}(n)$, are only distinguished by their values on the corresponding "critical" classes of cycle type $h(\lambda)=\left(h_{1}^{\lambda}, \ldots, h_{d}^{\lambda}\right)$, where $h_{1}^{\lambda}, \ldots, h_{d}^{\lambda}$ are the principal hook lengths in $\lambda$ and $d=d(\lambda)$ is the diagonal length of $\lambda$. Note that $h(\lambda) \in(\mathcal{O} \cap \mathscr{D})(n)$, so the corresponding $S_{n}$-class splits.

Then we have $[\lambda]\left(\sigma_{h(\lambda)}\right)=(-1)^{(n-d) / 2}=: \varepsilon_{\lambda}$. We set $H_{\lambda}=\prod_{i=1}^{d} h_{i}^{\lambda}$. Then

$$
\begin{aligned}
& \{\lambda\}_{+}\left(\sigma_{h(\lambda)}^{ \pm}\right)=\frac{1}{2}\left(\varepsilon_{\lambda} \pm \sqrt{\varepsilon_{\lambda} H_{\lambda}}\right), \\
& \{\lambda\}_{-}\left(\sigma_{h(\lambda)}^{ \pm}\right)=\frac{1}{2}\left(\varepsilon_{\lambda} \mp \sqrt{\varepsilon_{\lambda} H_{\lambda}}\right) .
\end{aligned}
$$

For any other irreducible $A_{n}$-character the values on these two classes coincide.

We have the following easy and well known property:

Lemma 4.1. The map $h: \mathscr{S}(n) \rightarrow(\mathcal{O} \cap \mathscr{D})(n)$ with $h(\lambda)=\left(h_{1}^{\lambda}, \ldots, h_{d(\lambda)}^{\lambda}\right)$, for $\lambda \in \mathscr{Y}(n)$, is a bijection. 
Let $B \in \mathrm{Bl}\left(S_{n}\right)$ with 2-core $\rho_{k}=(k, k-1, \ldots, 2,1)=\mathrm{dbl}\left(\tau_{k}\right)$, where, as before, $\tau_{k}=(2 k-1,2 k-5, \ldots)$; let $\mathscr{D}_{\widetilde{B}}$ and $\mathscr{O}_{B}=\mathscr{O}_{\widetilde{B}}$ as before in Section 3 and $(\mathscr{O} \cap \mathscr{D})_{\widetilde{B}}=$ $0_{\widetilde{B}} \cap \mathscr{D}_{\widetilde{B}}$.

We set $\mathscr{Y}_{B}=\left\{\lambda \in \mathscr{Y}(n) \mid \lambda_{(2)}=\rho_{k}\right\}$.

In our context, we need the following refinement of Lemma 4.1:

Proposition 4.2. The map $h$ induces bijections $\mathscr{I}_{B} \rightarrow(\mathscr{O} \cap \mathscr{D})_{\widetilde{B}}$.

Proof. We have to show that for any $\lambda \in \mathscr{S}(n)$, we have $\lambda_{(2)}=\operatorname{dbl}(h(\lambda))_{(2)}$. In the notation of $\overline{4}$-combinatorics an easy reduction argument shows that $\lambda_{(2)}=$ $\operatorname{dbl}\left(h(\lambda)_{(\overline{4})}\right)$; simultaneously removing 2-hooks from the diagram of $\lambda$ that are symmetrically positioned in $\lambda$ corresponds to removing 4-bars from $h(\lambda)$, namely, subtracting 4 from a part in $h(\lambda)$, and removing an inner $2 \times 2$ array corresponds to removing a pair 3,1 (which is also a 4-bar). This ends at a staircase partition $\rho_{k}=\lambda_{(2)}$, and in parallel at the corresponding $\tau_{k}=h(\lambda)_{(\overline{4})}$.

By [Bessenrodt and Olsson 1997, Lemma 3.6], we obtain the equation on the 2-cores.

Remark 4.3. It is not difficult to see [Olsson 1993, 12.5] that

$$
\left|\mathscr{S}_{B}\right|= \begin{cases}0 & \text { if } w(B) \text { is odd } \\ p(w / 2) & \text { if } w(B) \text { is even. }\end{cases}
$$

For a character $\chi$ of $G$, let $\chi^{o}$ denote the restriction of $\chi$ to the 2-regular elements of $G$. The following useful proposition provides a good 2-basic set for the alternating groups. Note here that the set $(\mathscr{D} \cap \mathscr{Y})(n)$ labeling the third subset of characters is nonempty only if $n$ is a triangular number; in that case, if $n=\left(\begin{array}{c}k+1 \\ 2\end{array}\right)$, $(\mathscr{D} \cap \mathscr{Y})(n)=\left\{\rho_{k}\right\}$, and thus both characters $\left\{\rho_{k}\right\}_{ \pm}$of defect 0 then belong to the basic set.

\section{Proposition 4.4. Set}

$$
\mathscr{C}(n)=\{\{\lambda\} \mid \lambda \in(\mathscr{D} \backslash \mathscr{Y})(n)\} \cup\left\{\{\lambda\}_{+} \mid \lambda \in \mathscr{Y}(n)\right\} \cup\left\{\{\lambda\}_{-} \mid \lambda \in(\mathscr{D} \cap \mathscr{S})(n)\right\} .
$$

Then $\left\{\chi^{o} \mid \chi \in \mathscr{C}(n)\right\}$ is a basic set for $A_{n}$ (at the prime $p=2$ ).

Proof. If $\chi=\{\mu\}=[\mu] \downarrow_{A_{n}}$ for $\mu \in(P \backslash \mathscr{S})(n)$, then, since the 2-regular partitions label a basic set for $S_{n}$,

$$
\chi^{o}=[\mu]^{o} \downarrow_{A_{n}}=\sum_{\lambda \in \mathscr{D}(n)} c_{\mu \lambda}[\lambda]^{o} \downarrow_{A_{n}}=\sum_{\lambda \in(\mathscr{D} \backslash \mathscr{S})(n)} c_{\mu \lambda}\{\lambda\}^{o}+\sum_{\lambda \in(\mathscr{D} \cap \mathscr{S})(n)} c_{\mu \lambda}\left(\{\lambda\}_{+}^{o}+\{\lambda\}_{-}^{o}\right),
$$

with integer coefficients $c_{\mu \lambda}$; as explained just before the proposition, the second sum above has at most one partition $\lambda \in(\mathscr{D} \cap \mathscr{S})(n)$ giving a contribution, namely when $n$ is a triangular number. 
For $\chi=\{\mu\}_{+}, \mu \in \mathscr{Y}(n)$, or $\chi=\{\mu\}_{-}, \mu \in(\mathscr{D} \cap \mathscr{S})(n)$, there is nothing to prove. If $\chi=\{\mu\}_{-}, \mu \in \mathscr{S} \backslash(\mathscr{D} \cap \mathscr{S})(n)$, then, again since the 2-regular partitions label a basic set for $S_{n}$,

$$
\begin{aligned}
\chi^{o} & =\{\mu\}_{-}^{o}=[\mu]^{o} \downarrow_{A_{n}}-\{\mu\}_{+}^{o}=\left(\sum_{\lambda \in \mathscr{D}(n)} c_{\mu \lambda}[\lambda]^{o} \downarrow_{A_{n}}\right)-\{\mu\}_{+}^{o} \\
& =\left(\sum_{\lambda \in(\mathscr{D} \backslash \mathscr{S})(n)} c_{\mu \lambda}\{\lambda\}^{o}+\sum_{\lambda \in(\mathscr{D} \cap \mathscr{S})(n)} c_{\mu \lambda}\left(\{\lambda\}_{+}^{o}+\{\lambda\}_{-}^{o}\right)\right)-\{\mu\}_{+}^{o},
\end{aligned}
$$

an integral linear combination as desired.

Since $|\mathscr{C}(n)|=|\mathscr{D}(n)|+|\mathscr{S}(n)|=|\mathscr{O}(n)|+|(\mathscr{O} \cap \mathscr{D})(n)|=\left|\mathrm{Cl}^{\prime}\left(A_{n}\right)\right|$, the set $\left\{\chi^{o} \mid \chi \in \mathscr{C}(n)\right\}$ is a $\mathbb{Z}$-basis of $\left\langle\chi^{o} \mid \chi \in \operatorname{Irr}\left(A_{n}\right)\right\rangle_{\mathbb{Z}}$, as claimed.

For any set $Q \subseteq P(n)$, we set

$$
a_{Q}=\prod_{\lambda \in Q} a_{\lambda} .
$$

Using the basic set above, we define the 2-regular character table for the alternating group $A_{n}$ to be

$$
X_{n}^{A}=(\chi(\sigma))_{\substack{\chi \in \mathscr{C}(n) \\ \sigma \in \mathscr{R}(n)}}
$$

Using the properties of the irreducible characters of $A_{n}$ stated above (see also [Bessenrodt and Olsson 2004]), as well as the formula for the 2-regular character table for $S_{n}$ from [Olsson 2003] we deduce:

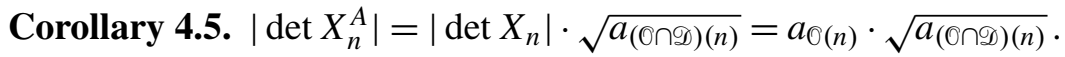

In the main theorem stated below, we will give a block refinement of the first equation above.

Remark 4.6. The 2-blocks of $S_{n}$ and $A_{n}$ are closely related [Olsson 1993] . Let $B \in \mathrm{Bl}\left(S_{n}\right)$. If $w(B)=0$, then $B$ covers two 2-blocks of $A_{n}$ (of defect 0 ), say $B_{\varepsilon}^{A}, \varepsilon \in\{ \pm\}$. This only occurs when $n$ is a triangular number, say $n=\left(\begin{array}{c}k+1 \\ 2\end{array}\right)$, and $\kappa(B)=\rho_{k}=(k, k-1, \ldots, 2,1)$; then $\operatorname{Irr}\left(B_{\varepsilon}^{A}\right)=\left\{\left\{\rho_{k}\right\}_{\varepsilon}\right\}, \varepsilon \in\{ \pm\}$.

Note that there is then a suitable choice of signs $\bar{\varepsilon}$ for $\varepsilon \in\{ \pm\}$ such that

$$
\left\{\rho_{k}\right\}_{\varepsilon}\left(\sigma_{h\left(\rho_{k}\right), \bar{\varepsilon}}\right) \not \equiv 0 \quad \bmod \mathfrak{p} .
$$

If $w(B)>0$, then $B$ covers only one 2-block $B^{A}$ of $A_{n}$, and this block $B^{A}$ is only covered by $B$. We then have

$$
\operatorname{Irr}\left(B^{A}\right)=\left\{\{\lambda\}_{( \pm)} \mid \lambda \in P(n), \lambda_{(2)}=\kappa(B)\right\} .
$$

Here, $\{\lambda\}_{( \pm)}$means that we take the character $\{\lambda\}$ if $\lambda$ is nonsymmetric, and both characters $\{\lambda\}_{ \pm}$if $\lambda$ is symmetric. 
Theorem 4.7. Let $B \in \mathrm{Bl}\left(S_{n}\right)$ with 2-core $\kappa(B)=\rho_{k}=\operatorname{dbl}\left(\tau_{k}\right)$.

If $w(B)=0$, then $\mathscr{O}_{B}=\left\{\tau_{k}\right\}$ and $\mathscr{D}_{B}=\left\{\rho_{k}\right\}$, and we set

$$
\mathrm{Cl}^{A}\left(B_{\varepsilon}^{A}\right)=\left\{\sigma_{\tau_{k}, \bar{\varepsilon}}^{A_{n}}\right\}, \quad \operatorname{Irr}^{\prime}\left(B_{\varepsilon}^{A}\right)=\left\{\left\{\rho_{k}\right\}_{\varepsilon}\right\}, \quad \text { for } \varepsilon \in\{ \pm\} .
$$

If $w(B)>0$, we set

$$
\mathrm{Cl}^{\prime A}\left(B^{A}\right)=\left\{\sigma_{\alpha(, \pm)}^{A_{n}} \mid \alpha \in \mathscr{O}_{B}\right\}, \quad \operatorname{Irr}^{\prime}\left(B^{A}\right)=\left\{\{\lambda\} \mid \lambda \in \mathscr{D}_{B}\right\} \cup\left\{\{\lambda\}_{+} \mid \lambda \in \mathscr{Y}_{B}\right\} .
$$

Let $X_{B^{A}}=\left(\chi\left(x_{K}\right)\right)_{\chi \in \operatorname{Irr}^{\prime}\left(B^{A}\right), K \in \mathrm{Cl}^{\prime A}\left(B^{A}\right)}$. Then

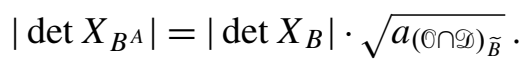

In particular, $\left|\operatorname{det} X_{B^{A}}\right| \not \equiv 0 \bmod \mathfrak{p}$. Hence, the sets above, taken for all $B \in$ $\mathrm{Bl}\left(S_{n}\right)$, define a 2-block splitting for $A_{n}$.

Proof. Using the notation above, let $B^{A}$ be a 2-block of $A_{n}$. As seen above, the sets $\mathrm{Cl}^{\prime A}\left(B^{A}\right)$ and $\operatorname{Irr}^{\prime}\left(B^{A}\right)$ are of the same cardinality. We have to show that all the block tables have a nonzero determinant modulo $\mathfrak{p}$.

For the case where $w(B)=0$, we have already seen before that we can make a suitable choice (namely the one used in the statement of the Theorem) such that this holds for the two blocks of $A_{n}$ covered by $B$.

Thus we may now assume that $w(B)>0$; then we do not have an irreducible character labeled by a partition of type $\mathscr{D} \cap \mathscr{Y}$ in $B$, that is, $\mathscr{D}_{B} \cap \mathscr{Y}_{B}=\varnothing$. We consider the part of the character table of $A_{n}$ corresponding to $B^{A}$, sorted such that among the character labels we first list the nonsymmetric ones and then the symmetric ones. The classes are ordered such that we first have the $\mathrm{O}_{B}$ classes, and among these classes the $(\mathscr{O} \cap \mathscr{D})_{\widetilde{B}}$ classes at the end, and here first the $(\mathscr{O} \cap \mathscr{D})_{\widetilde{B}}^{+}$ classes (where the "+" indicates that we take the representatives $\sigma_{\alpha}^{+}$), followed by the corresponding $(O \cap \mathscr{D})_{\widetilde{B}}^{-}$classes. The classes of type $(\mathscr{O} \cap \mathscr{D})_{\widetilde{B}}$ are taken in some ordering, and the $\mathscr{Y}_{B}$ characters are then taken in the corresponding order, that is, with the label $\mu \in \mathscr{Y}_{B}$ corresponding to $h(\mu) \in(\mathscr{O} \cap \mathscr{D})_{\widetilde{B}}$.

Recall that for any $\lambda \in(P \backslash \mathscr{S})(n)$ and $\alpha \in(\mathscr{O} \cap \mathscr{D})(n)$, we have $\{\lambda\}\left(\sigma_{\alpha}^{+}\right)=$ $\{\lambda\}\left(\sigma_{\alpha}^{-}\right)=[\lambda]\left(\sigma_{\alpha}\right)$. Now take $\mu \in \mathscr{Y}(n)$; then for $\alpha=h(\mu)$ we have

$$
\{\mu\}_{+}\left(\sigma_{h(\mu)}^{ \pm}\right)=\frac{1}{2}\left(\varepsilon_{\mu} \pm \sqrt{\varepsilon_{\mu} H_{\mu}}\right)=: y_{\mu}^{ \pm},
$$

while for $\beta \in(\mathscr{O} \cap \mathscr{D})(n), \beta \neq h(\mu)$, we have $\{\mu\}_{+}\left(\sigma_{\beta}^{+}\right)=\{\mu\}_{+}\left(\sigma_{\beta}^{-}\right)$. Set $c_{\mu}=$ $\sqrt{\varepsilon_{\mu} H_{\mu}}=y_{\mu}^{+}-y_{\mu}^{-}$. Now for any $\alpha \in(\mathscr{O} \cap \mathscr{D})_{\widetilde{B}}$, subtract the column of the block character table $X_{B^{A}}$ to the class of $\sigma_{\alpha}^{+}$from the one to the class of $\sigma_{\alpha}^{-}$. By the above, then the final columns to the $(\mathscr{O} \cap \mathscr{D})_{\widetilde{B}}^{-}$classes are transformed into an upper zero part, corresponding to the characters labeled by nonsymmetric partitions, and 
below this a diagonal matrix with diagonal entries $-c_{\mu}, \mu \in \mathscr{Y}_{B}$. The table

$$
X_{B}^{S}=\left(\{\lambda\}\left(\sigma_{\alpha}^{(+)}\right)\right)_{\substack{\lambda \in \mathscr{D}_{B} \\ \alpha \in \mathbb{O}_{\widetilde{B}}}}
$$

is the upper left hand block part of the table $X_{B^{A}}$. By the above and Section 3, this is exactly the block part of the 2-regular character table of the symmetric group corresponding to the block $B$ of $S_{n}$, with the block splitting constructed for the symmetric groups, that is,

$$
X_{B}^{S}=X_{B}=\left([\lambda]\left(\sigma_{\alpha}\right)\right)_{\substack{\lambda \in \mathscr{D}_{B} \\ \alpha \in \mathbb{O}_{B}}}
$$

Thus we have $\operatorname{det} X_{B}^{S} \not \equiv 0 \bmod \mathfrak{p}$. Hence

$$
\left|\operatorname{det} X_{B^{A}}\right|=\left|\operatorname{det} X_{B}^{S}\right| \cdot \prod_{\mu \in \mathscr{T}_{B}}\left|c_{\mu}\right|=\left|\operatorname{det} X_{B}\right| \cdot \sqrt{a_{(0 \cap \mathscr{D})_{\widetilde{B}}}} \neq \equiv 0 \quad \bmod \mathfrak{p},
$$

and we have proved that our construction provides a 2-block splitting for $A_{n}$.

Remark 4.8. In contrast to the case of symmetric groups, the block splitting of the 2-regular classes for the alternating groups as given above is not the only block splitting; already $A_{6}$ provides a counterexample. Indeed, instead of associating the classes to $\left(1^{6}\right),\left(1^{3}, 3\right),\left(3^{2}\right)$ to the principal 2-block of $A_{6}$, the choices $\left(1^{6}\right)$, $\left(1^{3}, 3\right),(1,5)_{+}$or $\left(1^{6}\right),\left(3^{2}\right),(1,5)_{+}$are also possible.

Recall that for $\alpha \in \mathscr{O}(n) \backslash(\mathscr{O} \cap \mathscr{D})(n)$, the corresponding conjugacy class of $\sigma_{\alpha}$ is nonsplit in $A_{n}$, so we then have $\left|C_{A_{n}}\left(\sigma_{\alpha}\right)\right|_{2}=2^{k_{\alpha}-1}$, with $k_{\alpha}=l(\alpha)-l(G(\alpha))$ as before.

By Brauer's Theorem 2.1 we can now deduce from our 2-block splitting given in Theorem 4.7 the following result on the Cartan matrices of 2-blocks of alternating groups, providing a combinatorial formula for the elementary divisors which is easy to compute.

Corollary 4.9. Let $B \in \mathrm{Bl}\left(S_{n}\right)$ of weight $w(B)>0$, covering the block $B^{A} \in$ $\mathrm{Bl}\left(A_{n}\right)$. Then the elementary divisors of the Cartan matrix $C_{B^{A}}$ are

$$
\left|C_{A_{n}}\left(\sigma_{\alpha}\right)\right|_{2}=2^{k_{\alpha}-1}, \quad \text { for } \alpha \in \mathscr{O}_{B} \backslash(\mathcal{O} \cap \mathscr{D})_{\widetilde{B}} ; \quad 1^{2\left|(\mathscr{O} \mathscr{D})_{\tilde{B}}\right|} .
$$

In particular,

$$
\operatorname{det} C_{B}=2^{2 \ell(B)-\ell\left(B^{A}\right)} \operatorname{det} C_{B^{A}} .
$$

\section{References}

[Bessenrodt 2007] C. Bessenrodt, "The 2-block splitting in symmetric groups", Algebra Number Theory 1:2 (2007), 223-238. MR 2009a:20021 Zbl 1160.20010

[Bessenrodt and Olsson 1997] C. Bessenrodt and J. B. Olsson, "The 2-blocks of the covering groups of the symmetric groups", Adv. Math. 129:2 (1997), 261-300. MR 98i:20011 Zbl 0979.20014 
[Bessenrodt and Olsson 2000] C. Bessenrodt and J. B. Olsson, "Spin representations and powers of 2”, Algebr. Represent. Theory 3:3 (2000), 289-300. MR 2001h:20009 Zbl 1037.20011

[Bessenrodt and Olsson 2004] C. Bessenrodt and J. B. Olsson, "On character tables related to the alternating groups”, Sém. Lothar. Combin. 52 (2004), Art. B52c. MR 2006a:20021

[Brauer 1956] R. Brauer, "Zur Darstellungstheorie der Gruppen endlicher Ordnung”, Math. Z. 63 (1956), 406-444. MR 17,824g Zbl 0093.03101

[Glaisher 1883] J. W. L. Glaisher, "A theorem in partitions", Messenger of Math. 12 (1883), 158170.

[James and Kerber 1981] G. James and A. Kerber, The representation theory of the symmetric group, Encyclopedia of Mathematics and its Applications 16, Addison-Wesley Publishing Co., Reading, MA, 1981. MR 83k:20003 Zbl 0491.20010

[Olsson 1986] J. B. Olsson, "Lower defect groups in symmetric groups", J. Algebra 104:1 (1986), 37-56. MR 87m:20039 Zbl 0604.20016

[Olsson 1993] J. B. Olsson, Combinatorics and representations of finite groups, Vorlesungen aus dem Fachbereich Mathematik 20, Universität Essen, 1993. MR 95b:20020 Zbl 0796.05095

[Olsson 2003] J. B. Olsson, "Regular character tables of symmetric groups", Electron. J. Combin. 10 (2003), Note 3. MR 2004c:20021

[Uno and Yamada 2006] K. Uno and H.-F. Yamada, "Elementary divisors of Cartan matrices for symmetric groups", J. Math. Soc. Japan 58:4 (2006), 1031-1036. MR 2007i:20020 Zbl 1108.20009

Communicated by Dave Benson

Received 2008-12-09 Revised 2009-08-04 Accepted 2009-08-05

bessen@math.uni-hannover.de Institut für Algebra, Zahlentheorie und Diskrete Mathematik, Fakultät für Mathematik und Physik, Leibniz Universität Hannover, Welfengarten 1, D-30167 Hannover, Germany http://www-ifm.math.uni-hannover.de/ bessen/ 


\section{Guidelines for Authors}

Authors may submit manuscripts in PDF format on-line at the Submission page at the ANT website.

Originality. Submission of a manuscript acknowledges that the manuscript is original and and is not, in whole or in part, published or under consideration for publication elsewhere. It is understood also that the manuscript will not be submitted elsewhere while under consideration for publication in this journal.

Language. Articles in ANT are usually in English, but articles written in other languages are welcome.

Required items. A brief abstract of about 150 words or less must be included. It should be self-contained and not make any reference to the bibliography. If the article is not in English, two versions of the abstract must be included, one in the language of the article and one in English. Also required are keywords and subject classifications for the article, and, for each author, postal address, affiliation (if appropriate), and email address.

Format. Authors are encouraged to use $\mathrm{LT}_{\mathrm{E}} \mathrm{X}$ but submissions in other varieties of $\mathrm{T}_{\mathrm{E}} \mathrm{X}$, and exceptionally in other formats, are acceptable. Initial uploads should be in PDF format; after the refereeing process we will ask you to submit all source material.

References. Bibliographical references should be complete, including article titles and page ranges. All references in the bibliography should be cited in the text. The use of $\mathrm{BibT}_{\mathrm{E}} \mathrm{X}$ is preferred but not required. Tags will be converted to the house format, however, for submission you may use the format of your choice. Links will be provided to all literature with known web locations and authors are encouraged to provide their own links in addition to those supplied in the editorial process.

Figures. Figures must be of publication quality. After acceptance, you will need to submit the original source files in vector graphics format for all diagrams in your manuscript: vector EPS or vector PDF files are the most useful.

Most drawing and graphing packages (Mathematica, Adobe Illustrator, Corel Draw, MAT$\mathrm{LAB}$, etc.) allow the user to save files in one of these formats. Make sure that what you are saving is vector graphics and not a bitmap. If you need help, please write to graphics@mathscipub.org with details about how your graphics were generated.

White Space. Forced line breaks or page breaks should not be inserted in the document. There is no point in your trying to optimize line and page breaks in the original manuscript. The manuscript will be reformatted to use the journal's preferred fonts and layout.

Proofs. Page proofs will be made available to authors (or to the designated corresponding author) at a Web site in PDF format. Failure to acknowledge the receipt of proofs or to return corrections within the requested deadline may cause publication to be postponed. 


\section{Algebra \& Number Theory}

Volume $3 \quad$ No. $7 \quad 2009$

Cox rings of degree one del Pezzo surfaces

Damiano Testa, Anthony Várilly-Alvarado and Mauricio Velasco

Hilbert schemes of 8 points

Dustin A. Cartwright, Daniel Erman, Mauricio Velasco and Bianca VIRAY

Discretely ordered groups

Peter A Linnell, Akbar Rhemtulla and Dale P. O. Rolfsen

The half-twist for $U_{q}(\mathfrak{g})$ representations

NoAh SNyder and PETER TINGLEY

A 2-block splitting in alternating groups 